\title{
Development of a Multi-Disciplinary Design Tool for Axial Flow Turbines
}

\author{
by \\ Stephen Kenny \\ B.Eng. (Aerospace)

\begin{abstract}
A thesis submitted to
the Faculty of Graduate Studies and Research

in partial fulfillment of the requirements

for the degree of
\end{abstract}

\section{Master of Applied Science}

in

Aerospace Engineering

Ottawa-Carleton Institute For

Mechanical and Aerospace Engineering

Department of Mechanical and Aerospace Engineering

Carleton University

Ottawa, Ontario, Canada

August 2005

(C) 2005

Stephen Kenny 


$\begin{array}{ll}\begin{array}{l}\text { Library and } \\ \text { Archives Canada }\end{array} & \begin{array}{l}\text { Bibliothèque et } \\ \text { Archives Canada }\end{array} \\ \begin{array}{l}\text { Published Heritage } \\ \text { Branch }\end{array} & \begin{array}{l}\text { Direction du } \\ \text { Patrimoine de l'édition }\end{array} \\ \begin{array}{l}\text { 395 Wellington Street } \\ \text { Ottawa ON K1A ON4 } \\ \text { Canada }\end{array} & \begin{array}{l}\text { 395, rue Wellington } \\ \text { Ottawa ON K1A ON4 } \\ \text { Canada }\end{array}\end{array}$

Your file Votre référence ISBN: 0-494-10089-3

Our file Notre référence

ISBN: 0-494-10089-3

NOTICE:

The author has granted a nonexclusive license allowing Library and Archives Canada to reproduce, publish, archive, preserve, conserve, communicate to the public by telecommunication or on the Internet, loan, distribute and sell theses worldwide, for commercial or noncommercial purposes, in microform, paper, electronic and/or any other formats.

The author retains copyright ownership and moral rights in this thesis. Neither the thesis nor substantial extracts from it may be printed or otherwise reproduced without the author's permission.
AVIS:

L'auteur a accordé une licence non exclusive permettant à la Bibliothèque et Archives Canada de reproduire, publier, archiver, sauvegarder, conserver, transmettre au public par télécommunication ou par l'Internet, prêter, distribuer et vendre des thèses partout dans le monde, à des fins commerciales ou autres, sur support microforme, papier, électronique et/ou autres formats.

L'auteur conserve la propriété du droit d'auteur et des droits moraux qui protège cette thèse. $\mathrm{Ni}$ la thèse ni des extraits substantiels de celle-ci ne doivent être imprimés ou autrement reproduits sans son autorisation.
In compliance with the Canadian

Privacy Act some supporting forms may have been removed from this thesis.

While these forms may be included in the document page count, their removal does not represent any loss of content from the thesis.
Conformément à la loi canadienne sur la protection de la vie privée, quelques formulaires secondaires ont été enlevés de cette thèse.

Bien que ces formulaires aient inclus dans la pagination, il n'y aura aucun contenu manquant. 
The undersigned recommend to

the Faculty of Graduate Studies and Research acceptance of the thesis

Development and Validation of a Comprehensive Design Tool for Axial Flow Turbines

submitted by

Stephen Kenny, B.Eng.

in partial fulfillment of the degree of

Master of Applied Science (Aerospace Engineering)

Thesis Co-supervisor

Thesis Co-supervisor

Chair, Department of Mechanical and Aerospace Engineering

Carleton University

August 2005 


\section{Abstract}

A preliminary design tool has been created to aid in the development and design of axial flow turbines. The design tool outputs all of the required geometry and flow conditions for the preliminary design of a single stage axial turbine. Inherent to the tool is its ability to produce performance estimates, both aerodynamically and structurally. The aerodynamic analysis is largely empirical based and makes use of the most up-todate correlations available in the literature. The tool is multi-disciplinary in its ability to produce structural results as well as aerodynamic results. First order estimates of the blade root and disk stresses, as well as fatigue and creep life predictions are conducted. Additionally, preliminary estimates of the minimum casing thickness for blade containment and the corresponding rotor burst speed are carried out. The tool has been created to obtain a fast estimate of performance and a fast screening of various design variables. The design tool is also required in order to produce a first geometrical input for more advanced computational fluid dynamic (CFD) and finite element method (FEM) analyses. A test case has been conducted through the design and development of two single stage turbines for a 1-MW gas turbine engine. The results of the design tool were then compared to those results obtained from complete CFD and FEM analyses in order to validate the accuracy of the tool. Overall, the results showed excellent agreement both aerodynamically and structurally. 


\section{Acknowledgements}

The author wishes to acknowledge the support of Professor J.E.D. Gauthier and Professor X. Huang in their efforts to make this thesis work possible. The author would also like to thank all of the faculty members of the Gas Turbine Fourth Year Project for their countless hours in preparing the students for real world experiences, particularly Professor J.E.D. Gauthier and Professor S.A. Sjolander. Without their efforts, much of this work could not have been completed. Finally, I would like to thank all of my colleagues, friends, and family for their endless support both academically and personally. 


\section{Table of Contents}

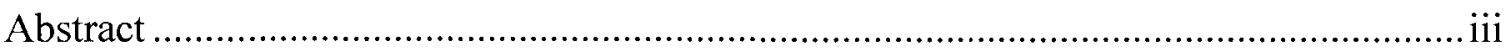

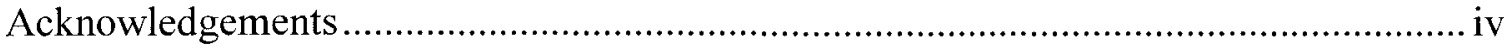

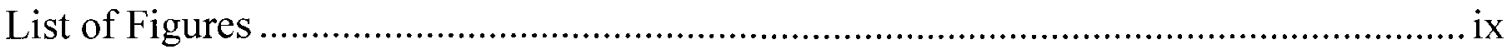

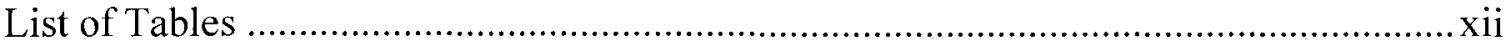

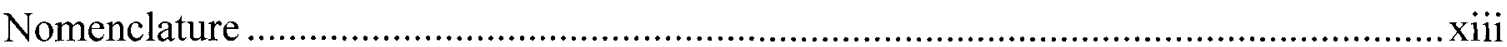

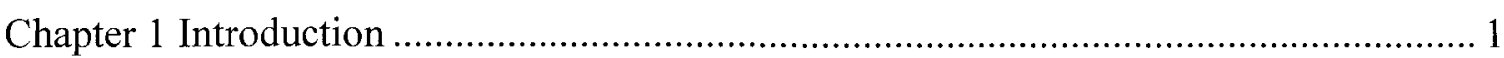

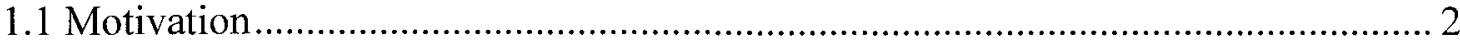

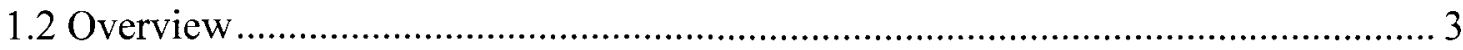

Chapter 2 Literature and Theoretical Reviews ......................................................... 5

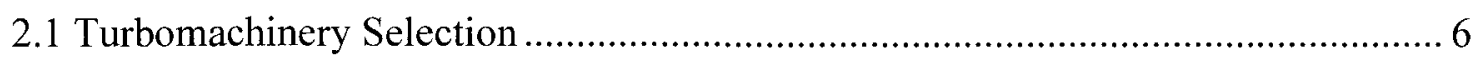

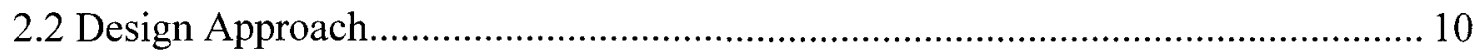

2.2.1 Preliminary Meanline Design ............................................................. 13

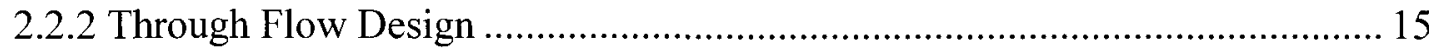

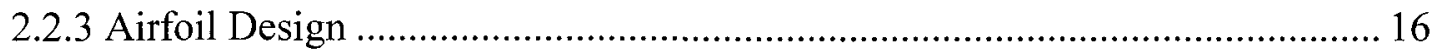

2.3 Turbomachinery Losses and Performance Evaluation................................... 21

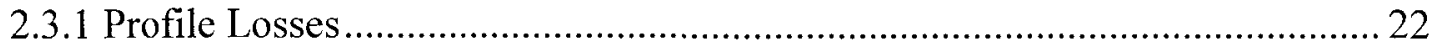

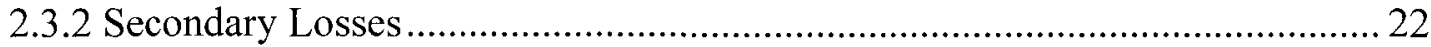

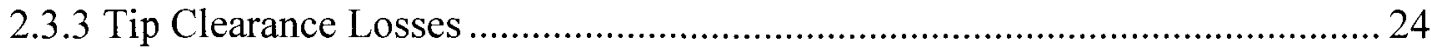

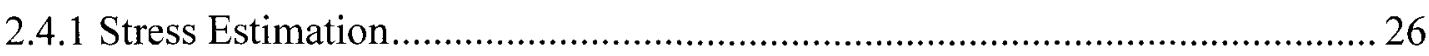

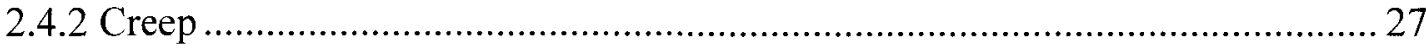

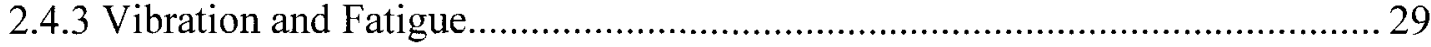

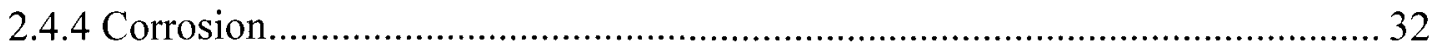

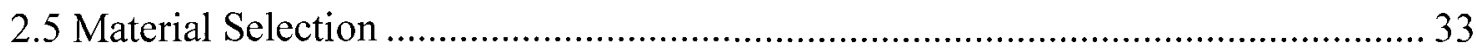

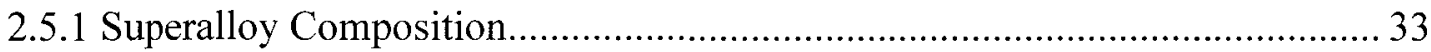

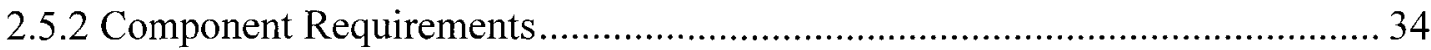

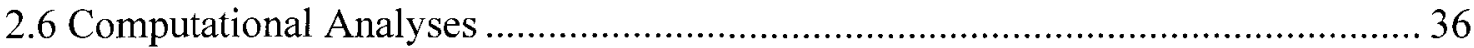

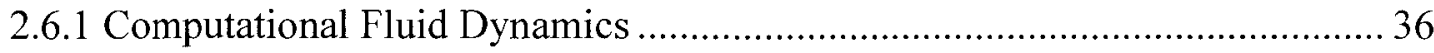

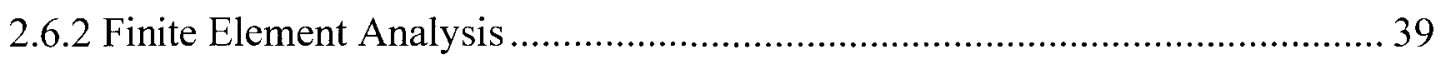

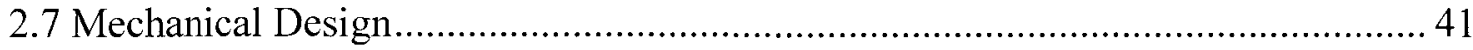


Chapter 3 Aerodynamic and Structural Design Tool.................................................. 43

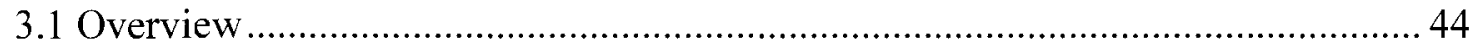

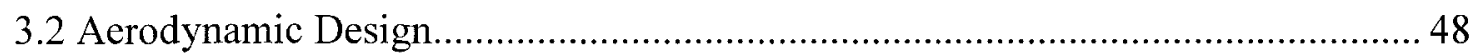

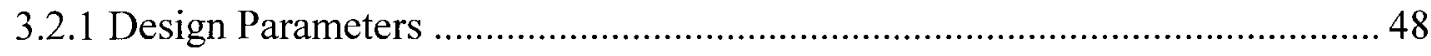

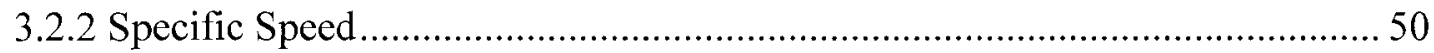

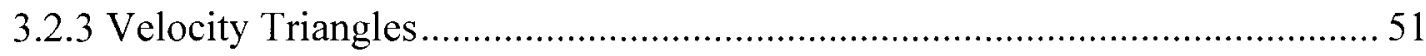

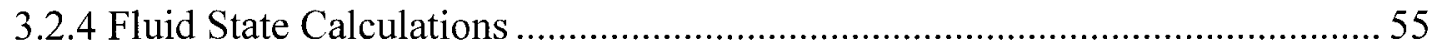

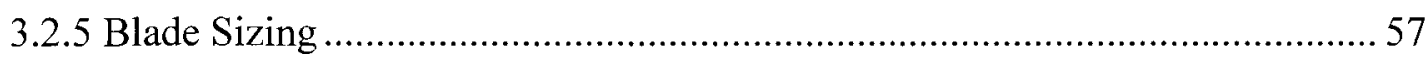

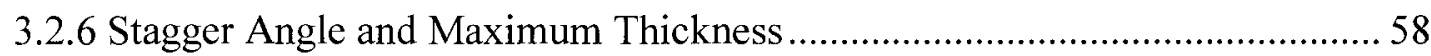

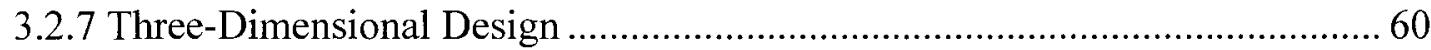

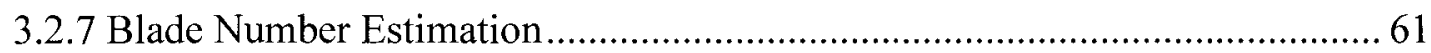

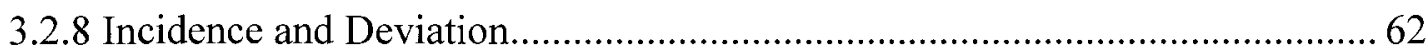

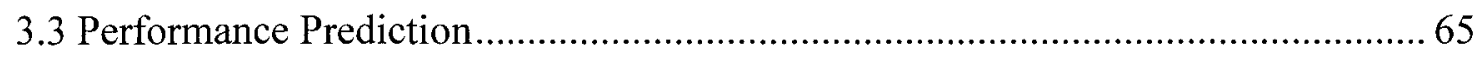

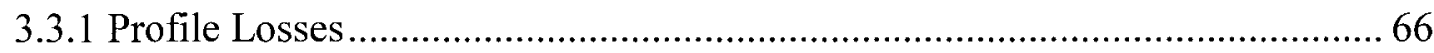

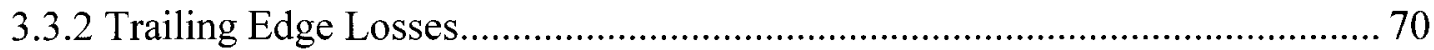

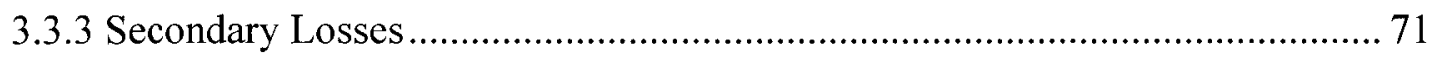

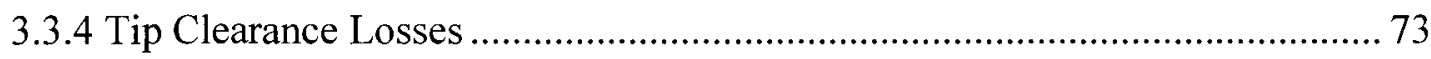

3.3.5 Total Pressure Loss Coefficient ...................................................... 73

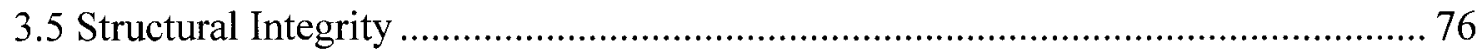

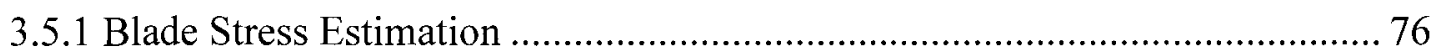

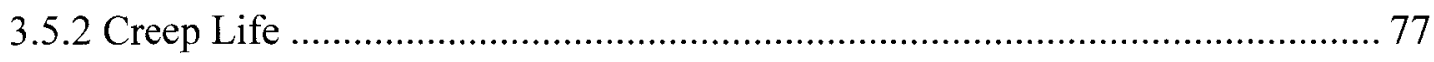

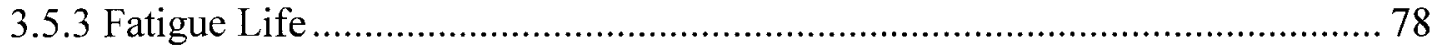

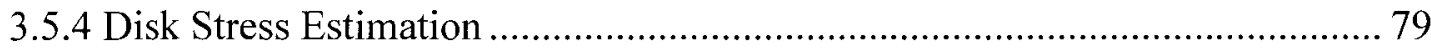

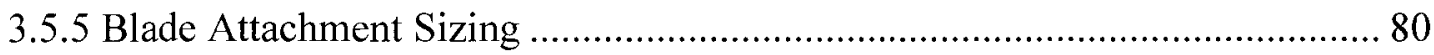

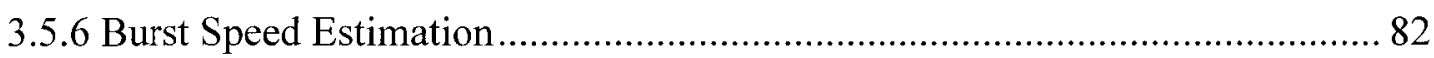

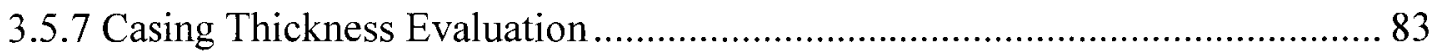

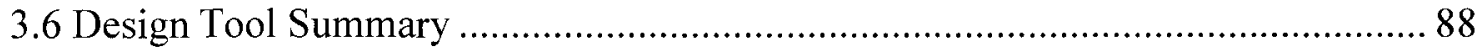

Chapter 4 Gas Generator and Power Turbine Design............................................... 91

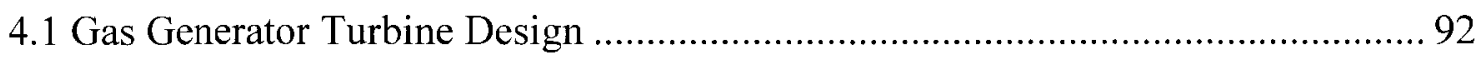

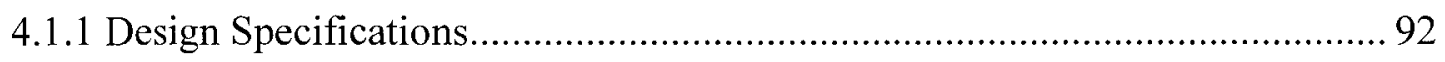

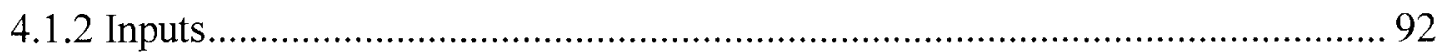




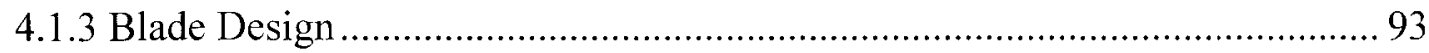

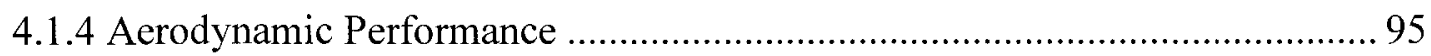

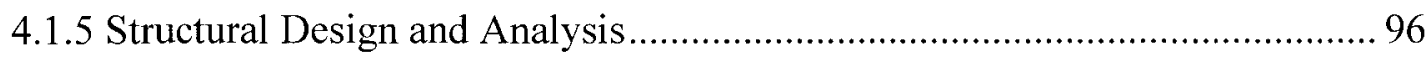

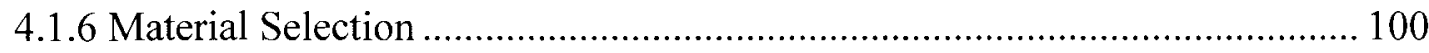

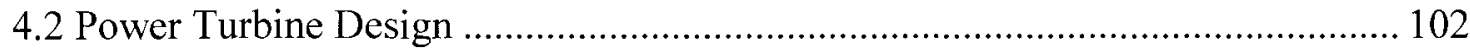

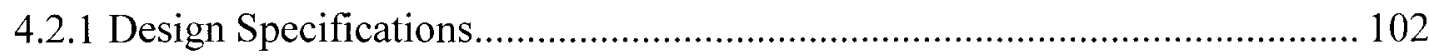

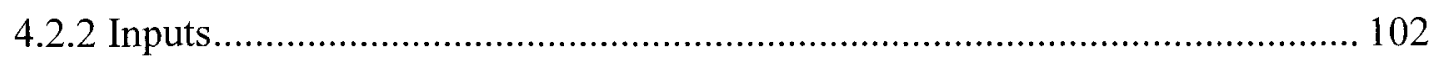

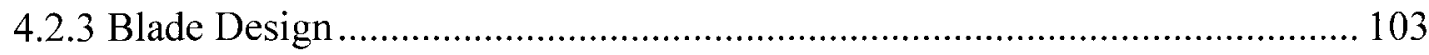

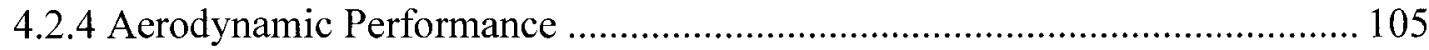

4.2.5 Structural Design and Analysis................................................................... 106

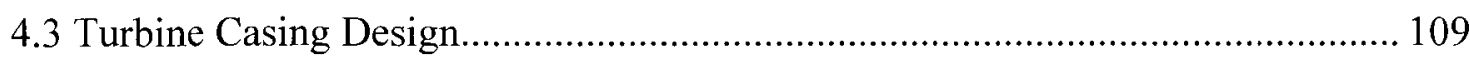

Chapter 5 Methods for Design Tool Validation............................................................. 111

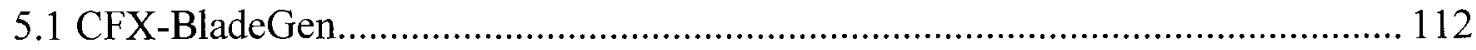

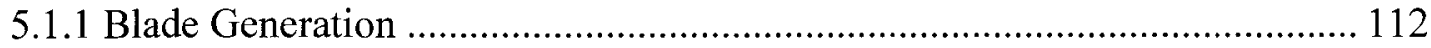

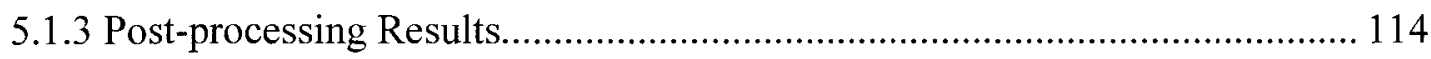

5.2 CFX-5

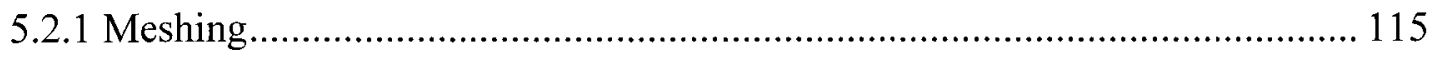

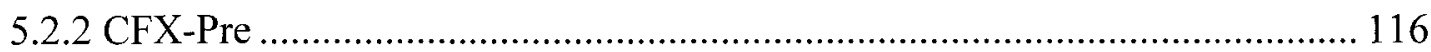

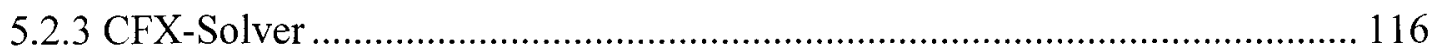

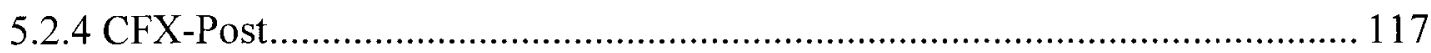

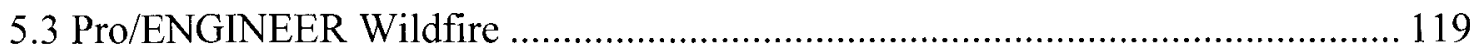

5.4 ANSYS 9.0

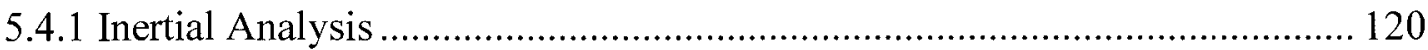

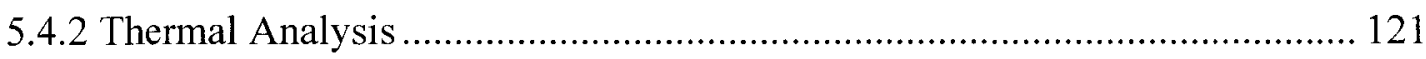

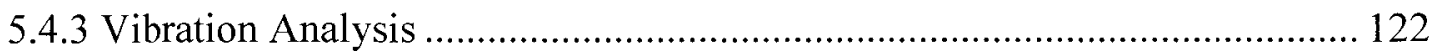

Chapter 6 Results and Discussion - CFD \& FEA ………………............................... 124

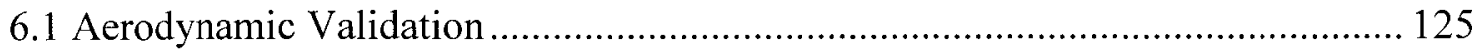

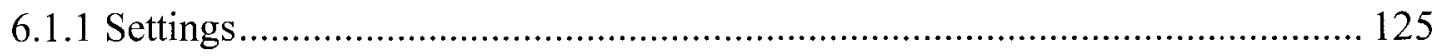

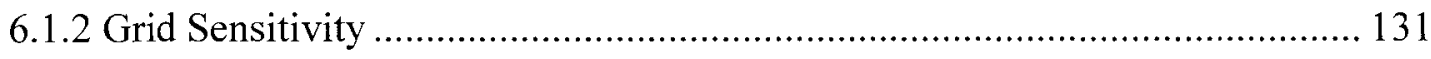

6.1.3 Aerodynamic Design Validation - Gas Generator Turbine ………………... 137

6.1.4 Aerodynamic Design Validation - Power Turbine ....................................... 144 


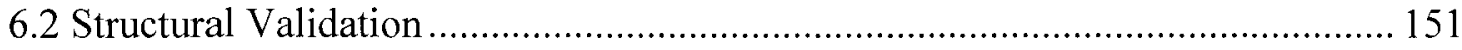

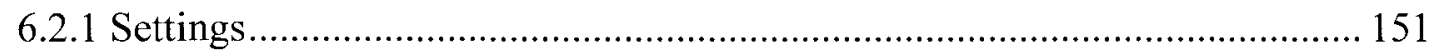

6.2.2 Structural Design Validation - Gas Generator Turbine …………………..... 154

6.2.3 Structural Design Validation - Power Turbine .............................................. 162

Chapter 7 Conclusions and Recommendations........................................................... 169

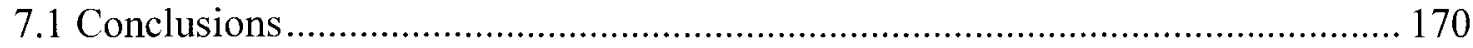

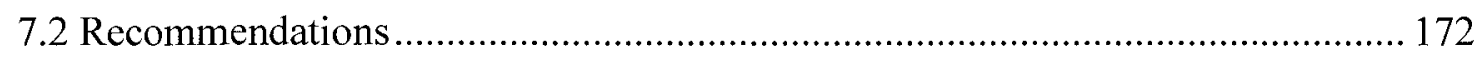

Appendix A1 - Aerodynamic Design Flow Chart ...................................................... 180

Appendix A2 - Performance Prediction Flow Chart ..................................................... 181

Appendix B1 - Design Tool Inputs ......................................................................... 182

Appendix B2 - Design Tool Outputs................................................................. 184

Appendix C1 - Curve Fits for Axial Turbine Design and Analysis ............................... 186

Appendix D1 - Blade Geometry with Tip Clearance in ANSYS Workbench 9.0 ......... 187

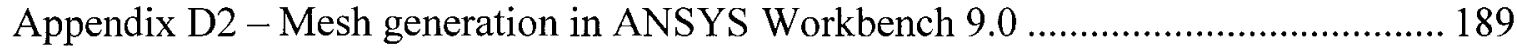

Appendix D3 - Stator-Rotor Simulation Setup in CFX-Pre........................................... 192

Appendix D4 - Creation of Rotor Solid Model in Pro/ENGINEER ………………….... 196

Appendix D5 - Model setup in ANSYS 9.0 ................................................................ 199

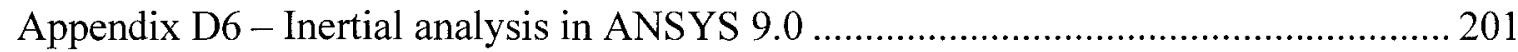

Appendix D7 - Thermal analysis in ANSYS 9.0 ………………………………..... 202

Appendix D8 - Vibration analysis in ANSYS 9.0........................................................ 204 


\section{List of Figures}

Figure 1.1: Schematic of turboshaft gas turbine engine............................................... 1

Figure 1.2: Schematic of gas generator and free power turbines ................................. 2

Figure 1.3: (a) Radial inflow turbine and (b) axial flow turbine.................................... 3

Figure 2.1: Efficiency versus specific speed for radial turbines ..................................... 7

Figure 2.2: Stage loading and flow coefficient for radial turbines ................................ 8

Figure 2.3: Maximum turbine efficiency as a function of specific speed....................... 9

Figure 2.4: Correlated specific speed and specific diameter for radial turbines for

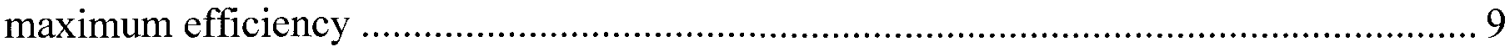

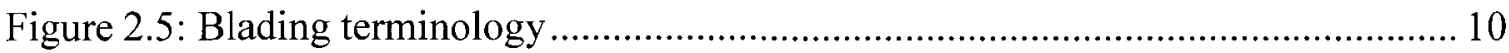

Figure 2.6: Smith chart for turbine stage efficiency ............................................ 11

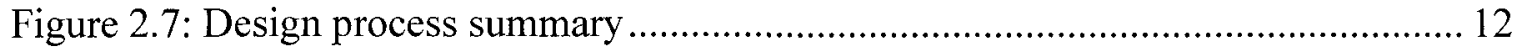

Figure 2.8: Velocity and pressure distribution for free-vortex design ........................... 15

Figure 2.9: Effect of incidence on profile loss...................................................... 17

Figure 2.10: Optimum blade spacing, Ainley's correlation...................................... 20

Figure 2.11: Schematic diagram of passage vortex formation..................................... 23

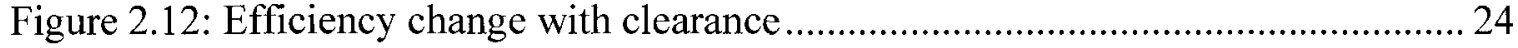

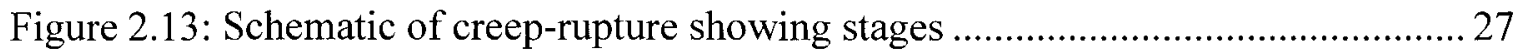

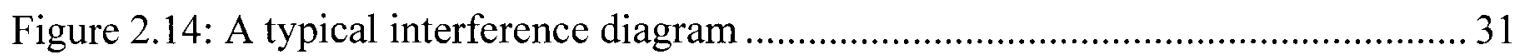

Figure 2.15: (a) Mach number distribution by CFD and (b) finite element mesh ........... 36

Figure 2.16: Allison T56 first stage turbine blade with fir-tree root.............................. 41

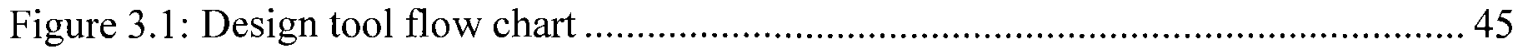

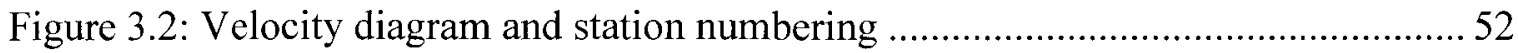

Figure 3.3: (a) Stagger angle for typical turbine blade sections

and (b) Thickness-to-chord ratio for typical turbine blade sections ............................. 59

Figure 3.4: Incidence, deviation, and throat opening conventions ............................ 63

Figure 3.5: (a) Profile loss coefficient for $\alpha^{\prime}{ }_{1}=0$

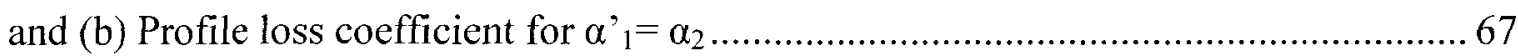

Figure 3.6: Accepted values and nomenclature for fir-tree design ......................... 81

Figure 4.1: Gas generator turbine meanline velocity triangles ................................... 94 
Figure 4.2: Variation in loss mechanisms for gas generator turbine design 96

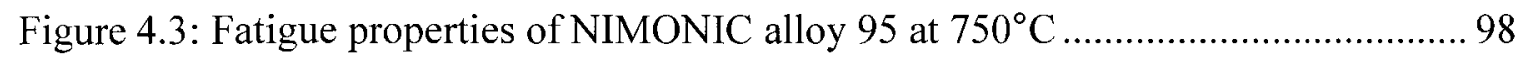

Figure 4.4: Preliminary gas generator turbine blade attachment design ....................... 99

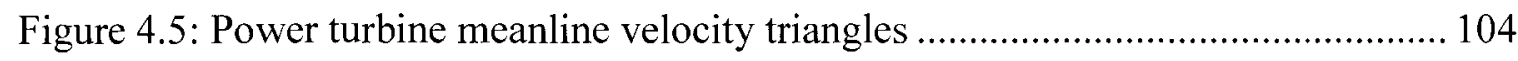

Figure 4.6: Variation in loss mechanisms for power turbine design ......................... 106

Figure 4.7: Preliminary power turbine blade attachment design ............................. 107

Figure 4.8: Proposed turbomachinery layout and casing design ................................ 109

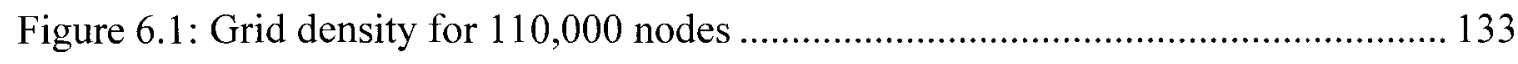

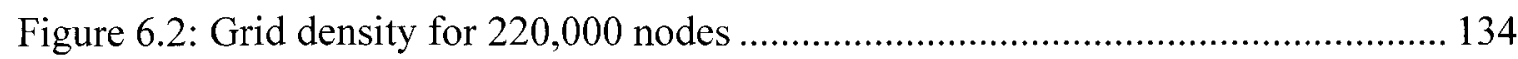

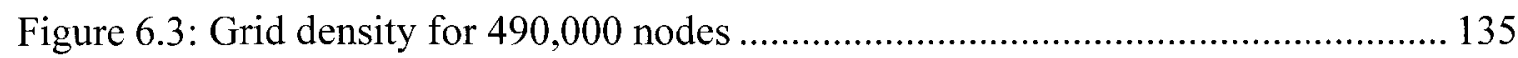

Figure 6.4: Localized effects of the grid refinement study .................................... 136

Figure 6.5: Stator blade loading (gas generator turbine) ......................................... 140

Figure 6.6: Rotor blade loading (gas generator turbine) ....................................... 140

Figure 6.7: Mach number distribution at gas generator turbine mid-span................... 141

Figure 6.8: (a) Vector diagram and (b) total pressure distribution

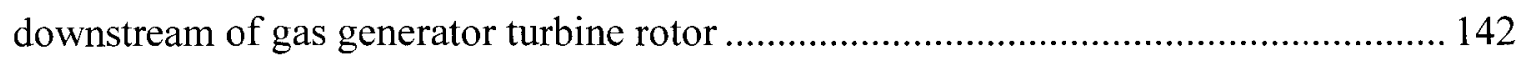

Figure 6.9: Gas generator turbine characteristics................................................ 143

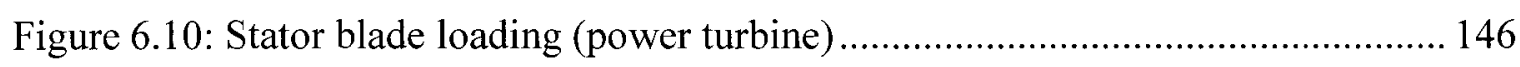

Figure 6.11: Rotor blade loading (power turbine) ............................................. 146

Figure 6.12: Mach number distribution at power turbine mid-span ........................... 147

Figure 6.13: (a) Vector diagram and (b) total pressure distribution

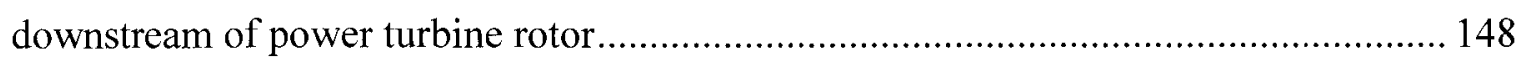

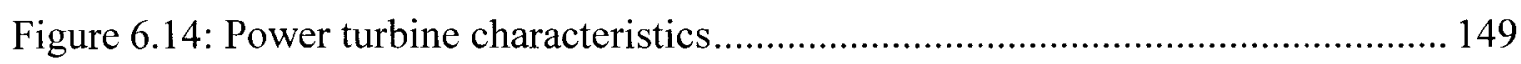

Figure 6.15: Gas generator turbine rotor finite element mesh ................................ 155

Figure 6.16: Rotor von Mises stress distribution (gas generator turbine)...................... 156

Figure 6.17: Blade von Mises stress distribution (gas generator turbine) ...................... 156

Figure 6.18: Rotor stress distribution - ANSYS vs design tool ............................... 157

Figure 6.19: Gas generator turbine rotor temperature distribution ............................ 158

Figure 6.20: Radial growth of gas generator turbine due to thermal effects ................. 159

Figure 6.21: Gas generator turbine blade finite element mesh ................................ 159

Figure 6.22: Blade root stress distribution (gas generator turbine) .......................... 160 
Figure 6.23: Gas generator turbine Campbell diagram................................................. 161

Figure 6.24: Power turbine finite element mesh ........................................................... 162

Figure 6.25: Rotor von Mises stress distribution (power turbine) …………………..... 163

Figure 6.26: Rotor stress distribution - ANSYS vs design tool ..................................... 164

Figure 6.27: Power turbine rotor temperature distribution ............................................ 165

Figure 6.28: Radial growth of power turbine rotor due to thermal effects ..................... 165

Figure 6.29: Power turbine blade finite element mesh ................................................ 166

Figure 6.30: Blade root stress distribution (power turbine) ........................................... 167

Figure 6.31: Campbell diagram for the power turbine assembly ................................... 167

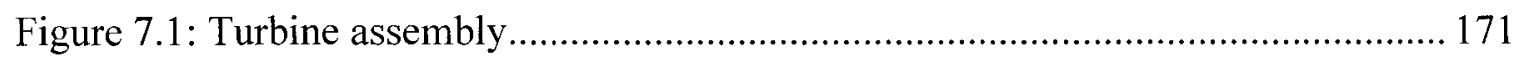

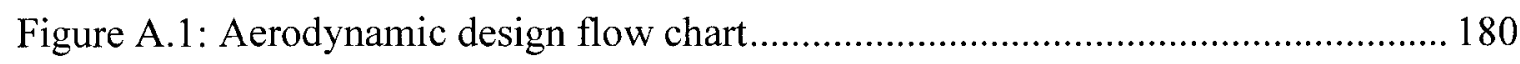

Figure A.2: Performance prediction flow chart ......................................................... 181 


\section{List of Tables}

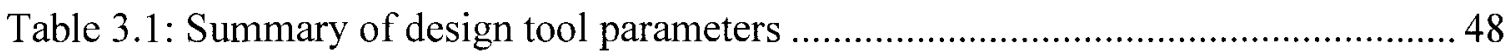

Table 3.2: Output station flow conditions and performance expectations........................ 88

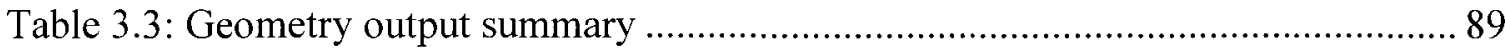

Table 4.1: Summary of gas generator turbine fluid states ............................................ 95

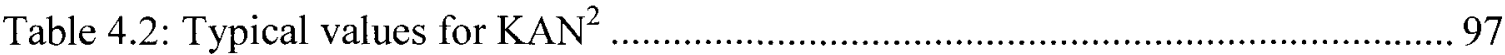

Table 4.3: Gas generator turbine disk stress variation ....................................................... 99

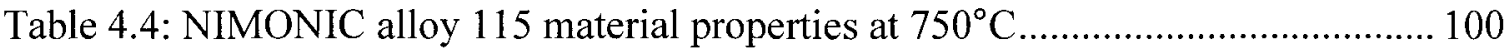

Table 4.5: Summary of power turbine fluid states....................................................... 105

Table 4.6: Power turbine disk stress variation .............................................................. 107

Table 5.1: CFX-BladeGenPlus analysis requirements.................................................... 113

Table 6.1: Summary of simulation settings used in CFX-5 .......................................... 131

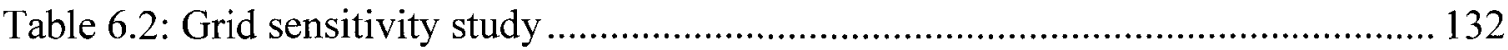

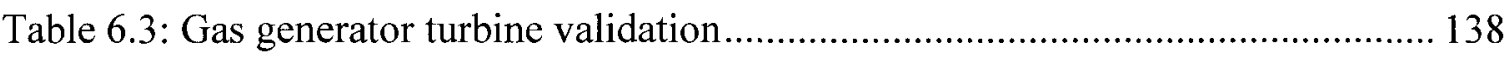

Table 6.4: Power turbine validation ........................................................................ 145

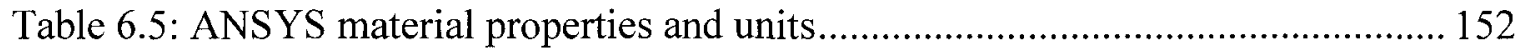

Table 6.6: Summary of inertia analysis settings ……………....................................... 153

Table 6.7: Summary of thermal analysis settings ....................................................... 153

Table 6.8: Summary of vibration analysis settings ..................................................... 154

Table B.1: Design parameter inputs...................................................................... 182

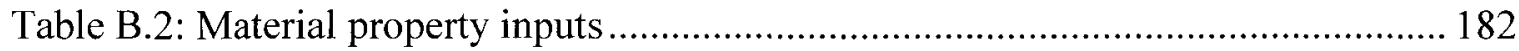

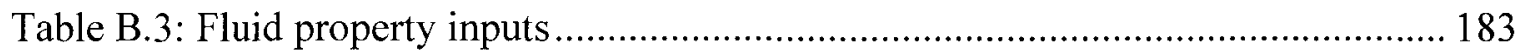

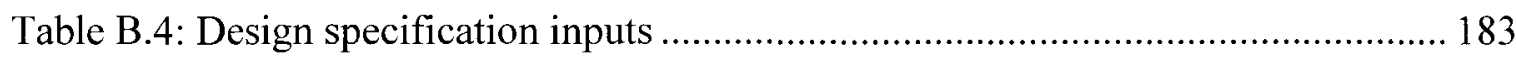

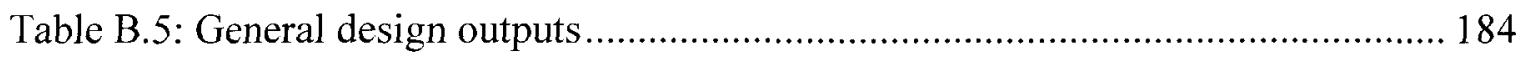

Table B.6: Additional station outputs .................................................................... 184

Table B.7: Stator and rotor outputs ............................................................................. 184

Table B.8: Performance estimation outputs .............................................................. 185

Table B.9: Structural evaluation outputs................................................................. 185 


\section{Nomenclature}

\begin{tabular}{|c|c|}
\hline$\% \mathrm{PL}$ & Percent stagnation pressure loss \\
\hline A & Area \\
\hline AMDC & Ainley, Mathieson, Dunham and Came \\
\hline AR & Aspect ratio \\
\hline A ratio & Area ratio \\
\hline B & Gas bending correlation \\
\hline $\mathrm{C}$ & Absolute velocity \\
\hline $\mathrm{c}$ & Chord \\
\hline $\mathrm{C}_{\mathrm{D}}$ & Discharge coefficient \\
\hline CFM & Supersonic drag rise \\
\hline $\mathrm{C}_{\mathrm{L}}$ & Zweifel lift coefficient \\
\hline$C_{p}$ & Specific heat \\
\hline $\mathrm{D}$ & Diameter \\
\hline $\mathrm{D}_{\mathrm{s}}$ & Specific diameter \\
\hline $\mathrm{E}_{\mathrm{c}}$ & Energy due to compression \\
\hline $\mathrm{E}_{\mathrm{s}}$ & Energy due to shear \\
\hline $\mathrm{F}_{\text {blade }}$ & Force on single blade \\
\hline$F_{\text {total }}$ & Total force on all blades \\
\hline $\mathrm{f}_{\mathrm{AR}}$ & Aspect ratio correction factor \\
\hline $\mathrm{f}_{\mathrm{Re}}$ & Reynolds number correction factor \\
\hline $\mathrm{h}$ & Blade height \\
\hline $\mathrm{h}_{0}$ & Total enthalpy \\
\hline HTR & Hub-to-tip ratio \\
\hline $\mathrm{i}$ & Incidence \\
\hline k & Tip clearance \\
\hline $\mathrm{KE}$ & Kinetic Energy \\
\hline $\mathrm{k}_{\mathrm{n}}, \mathrm{k}_{\mathrm{m}}$ & Correction factor defined in text \\
\hline $\mathrm{K}_{5}, \mathrm{~K}_{\mathrm{p}}, \mathrm{K}_{1}, \mathrm{~K}_{2}, \mathrm{~K}_{3}$ & Correction factor defined in text \\
\hline$K_{\text {taper }}$ & Blade taper ratio \\
\hline LM & Larsen-Miller parameter \\
\hline M & Mach number \\
\hline M1 & One third mass of rotor \\
\hline M2 & Mass of casing \\
\hline $\mathrm{m}_{\text {air }}$ & Mass flow rate of air \\
\hline $\mathrm{N}$ & Rotational speed, RPM \\
\hline $\mathrm{n}$ & Number of blades \\
\hline$n^{\prime}$ & Gas bending correlation \\
\hline $\mathrm{N}_{\mathrm{s}}$ & Specific speed, unitless \\
\hline
\end{tabular}




\begin{tabular}{|c|c|}
\hline o & Throat opening \\
\hline $\mathrm{P}$ & Pressure \\
\hline$P_{\text {shear }}$ & Shear perimeter \\
\hline Q & Volume flow rate \\
\hline$Q_{\text {mat }}$ & Volume of material \\
\hline $\mathrm{r}$ & Radius \\
\hline $\mathrm{R}$ & Average radius \\
\hline $\mathrm{R}_{\text {air }}$ & Gas constant of air \\
\hline $\mathrm{Re}$ & Reynolds number \\
\hline $\mathrm{r}_{\text {fragment }}$ & Radius to center of gravity of fragment \\
\hline $\mathrm{s}$ & Spacing \\
\hline $\mathrm{t}$ & Time \\
\hline$t_{\text {blade }}$ & Blade thickness \\
\hline$t_{\text {casing }}$ & Casing thickness \\
\hline $\mathrm{T}$ & Temperature \\
\hline $\mathrm{T}^{\prime}$ & Isentropic temperature \\
\hline $\operatorname{tmax} / \mathrm{c}$ & Thickness-to-chord ratio \\
\hline $\mathrm{t}_{\mathrm{TET}}$ & Trailing edge thickness \\
\hline $\mathrm{U}$ & Tangential blade speed \\
\hline$V_{\text {fragment }}$ & Velocity of fragment \\
\hline W & Relative velocity \\
\hline $\mathrm{w}_{\mathrm{sp}}$ & Specific work output \\
\hline$x_{n}$ & Blade attachment width at counter $n$ \\
\hline$x_{\mathrm{p}}$ & Blade attachment platform width \\
\hline Y & Pressure loss coefficient \\
\hline $\mathrm{y}_{\mathrm{n}}$ & Blade attachment depth at counter $n$ \\
\hline$Y_{P}$ & Profile pressure loss coefficient \\
\hline$y_{p}$ & Blade attachment platform depth \\
\hline$Y_{P}^{\prime}$ & Profile pressure loss coefficient of AMDC \\
\hline$Y_{S}$ & Secondary loss coefficient \\
\hline $\mathrm{Y}^{\prime} \mathrm{S}$ & Secondary loss coefficient of AMDC \\
\hline$Y_{\text {shock }}$ & Shock loss coefficient \\
\hline$Y_{\mathrm{T}}$ & Total pressure loss coefficient \\
\hline $\mathrm{y}_{\mathrm{t}}$ & Blade attachment total depth \\
\hline $\mathrm{Y}_{\mathrm{TC}}$ & Tip clearance pressure loss coefficient \\
\hline $\mathrm{Y}_{\mathrm{TET}}$ & Trailing edge pressure loss coefficient \\
\hline $\mathrm{z}$ & Gas bending correlation \\
\hline
\end{tabular}




\section{Greek Symbols}

\begin{tabular}{|c|c|}
\hline$\alpha$ & Inlet flow angle in stationary reference frame (from axial) \\
\hline$\alpha^{\prime}$ & Inlet metal angle in stationary reference frame (from axial) \\
\hline$\alpha_{\mathrm{m}}$ & Mean gas angle defined in text \\
\hline$\beta$ & Outlet flow angle in relative reference frame (from axial) \\
\hline$\beta^{\prime}$ & Outlet metal angle in relative reference frame (from axial) \\
\hline$\gamma$ & Ratio of specific heats \\
\hline$\Delta$ & Delta, or change in \\
\hline$\delta$ & Deviation \\
\hline$\Delta \varphi^{2} \mathrm{TET}$ & Trailing edge kinetic energy loss coefficient \\
\hline$\varepsilon$ & Strain \\
\hline$\varepsilon_{\mathrm{c}}$ & Compressive strain \\
\hline$\varepsilon_{\mathrm{f}}$ & Strain to fracture \\
\hline$\zeta$ & Blade attachment angle \\
\hline$\eta$ & Isentropic efficiency \\
\hline$\eta_{\text {to }}$ & Isentropic efficiency for zero tip clearance \\
\hline$\Lambda$ & Degree of reaction \\
\hline$\mu$ & Fluid viscosity \\
\hline$\rho$ & Density of gas \\
\hline$\rho_{\text {rotor }}$ & Rotor material density \\
\hline$\rho_{\text {casing }}$ & Casing material density \\
\hline$\sigma$ & Solidity \\
\hline$\sigma_{\mathrm{d}}$ & Dynamic material strength \\
\hline$\sigma_{\mathrm{gb}}$ & Gas bending stress \\
\hline$\sigma_{\mathrm{H}}$ & Blade root stress \\
\hline$\sigma_{\mathrm{r}}$ & Radial disk stress \\
\hline$\sigma_{\text {rim }}$ & Rim stress \\
\hline$\sigma_{\mathrm{t}}$ & Tangential disk stress \\
\hline$\sigma_{\mathrm{UTS}}$ & Ultimate tensile strength \\
\hline$\sigma_{\mathrm{vM}}$ & von Mises stress \\
\hline$\tau_{\mathrm{d}}$ & Dynamic shear strength \\
\hline$v$ & Poisson's ratio \\
\hline$\varphi$ & Flow coefficient \\
\hline$\Phi$ & Stagger angle \\
\hline$\chi$ & Fragment angle \\
\hline$\psi$ & Stage loading coefficient \\
\hline$\omega$ & Rotational speed, $\mathrm{rad} / \mathrm{s}$ \\
\hline
\end{tabular}




\section{Subscripts}

0

1

2

3

d

in

ex

h

$\mathrm{m}$

$\mathrm{t}$

$\mathrm{X}$

$\theta$

$\mathrm{r}$

$\mathrm{N}$

$\mathrm{R}$

rel

$\max$
Stagnation property

Stator inlet

Stator outlet, rotor inlet

Rotor outlet

Design specification

Blade row inlet

Blade row exit

Hub

Meanline

Tip

Axial component

Tangential component

Radial component

Designates stator (nozzle)

Designates rotor

Relative reference frame

Maximum quantity 


\section{Chapter 1}

\section{Introduction}

Current gas turbine technologies are increasingly pushing the limits of fuel efficiency, weight, compactness, and cost effectiveness. These technologies are allowing engine manufacturers to design and produce state-of-the-art engines of various sizes and outputs for specific disciplines. Conventionally, the gas turbine engine has been used primarily by the aircraft industry but further developments and environmental trends are warranting its use in the industrial setting. To date, numerous engines are available for use in aircraft application, power generation, and other industrial installations.

The overall goal of this project was to design a low-cost gas turbine engine for powerboat application amongst a small team of graduate students within the Department of Mechanical and Aerospace Engineering at Carleton University in Ottawa, Ontario. Gas turbine engines may assume various configurations depending on the layout of the turbomachinery, heat recovery systems, and desired application. For the present application, it was chosen to design a turboshaft engine with a free power turbine, shown in Figure 1.1.

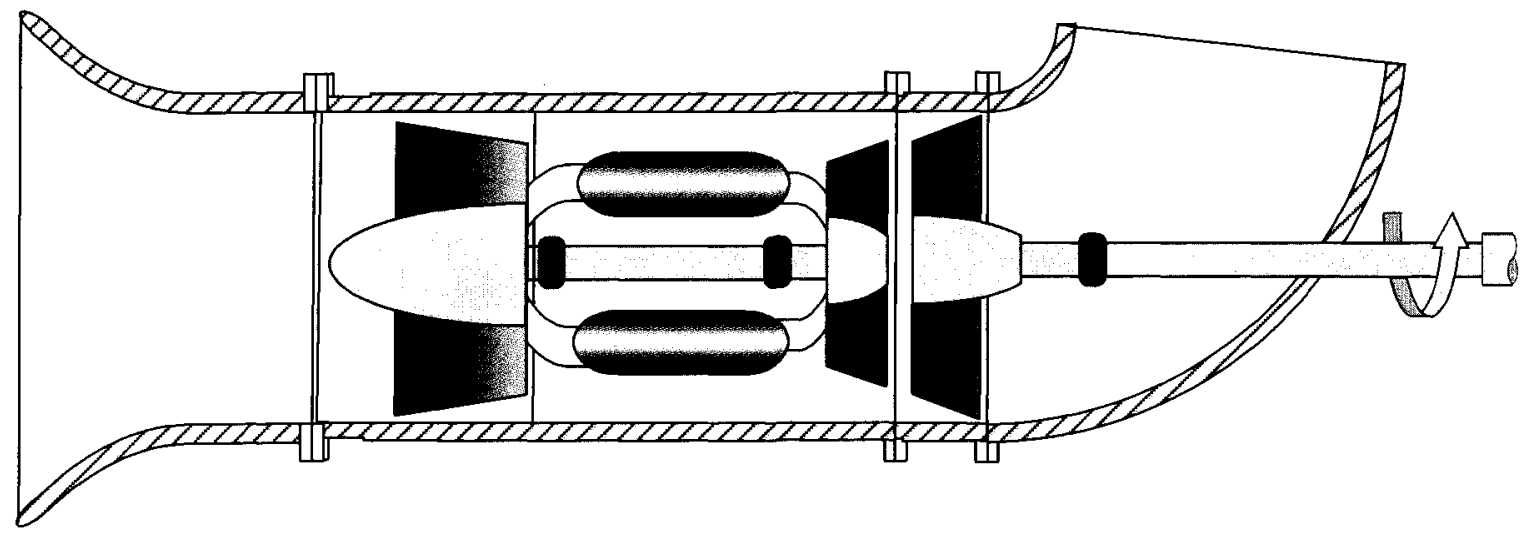

Figure 1.1: Schematic of turboshaft gas turbine engine 


\subsection{Motivation}

The compactness of the gas turbine engine makes it a suitable choice for this application over conventional inboard or outboard motors. To date, only gas turbine engines for use in helicopter power plants have been suitable for this use. These engines have been designed specifically for lightweight applications with airworthiness in mind; thus they are usually very expensive and not ideal for the current application.

The thesis focuses on the development of a comprehensive tool for the preliminary design of axial flow turbines. The tool is an empirical based design tool containing the latest correlations for aerodynamic performance predictions. The goal of this particular thesis is to produce a multi-disciplinary design tool capable of the initial screening and selection processes required for the preliminary design of turbine components. The tool will provide the initial steps required before further computational fluid dynamic and finite element optimization may take place. The tool is then applied in the development of two single-stage turbines in the design of 1-MW gas turbine engine. A schematic indicating the position of turbines within the engine is shown in Figure 1.2. The tasks required to complete the design will be summarized at the end of this chapter in order to provide a roadmap for the overall organization of the thesis.

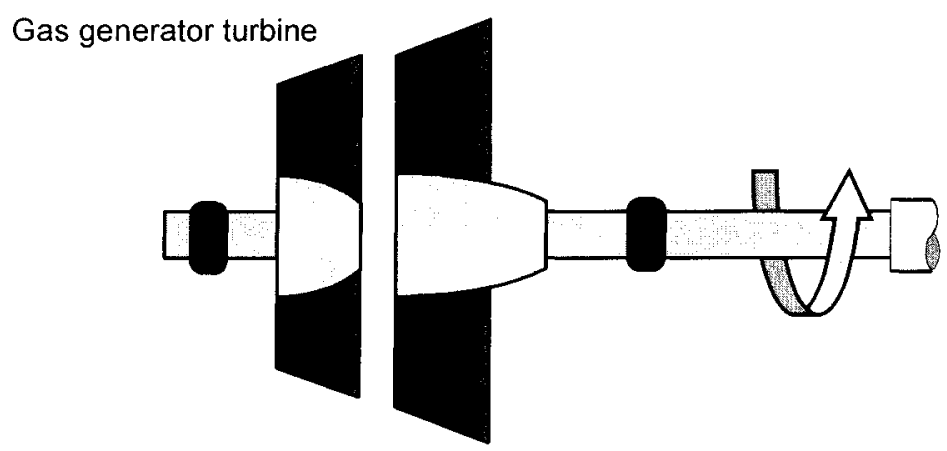

Free power turbine

Figure 1.2: Schematic of gas generator and free power turbines 


\subsection{Overview}

The development of this engine will involve the design of a complete gas generator turbine and a separate free power turbine. The scope of this thesis will concentrate on the aerodynamic and structural design of the turbine rotors, nozzles, and housings. Within the overall project, additional theses by other graduate students present the design of the other major components such as the compressor, combustion system, shaft and bearings, and control systems. These components are the responsibility of other members of the design team. The team is currently comprised of six graduate students, three co-supervisors (professors), and one industry sponsor.

The turbine rotor is designed to extract the energy from the hot gases downstream of the combustion chamber. Various sizes and configurations of turbines exist which are suitable to different design applications. Generally, turbine rotors are of the radial inflow or axial flow configuration, Figure 1.3(a) and 1.3(b), respectively.

(a)

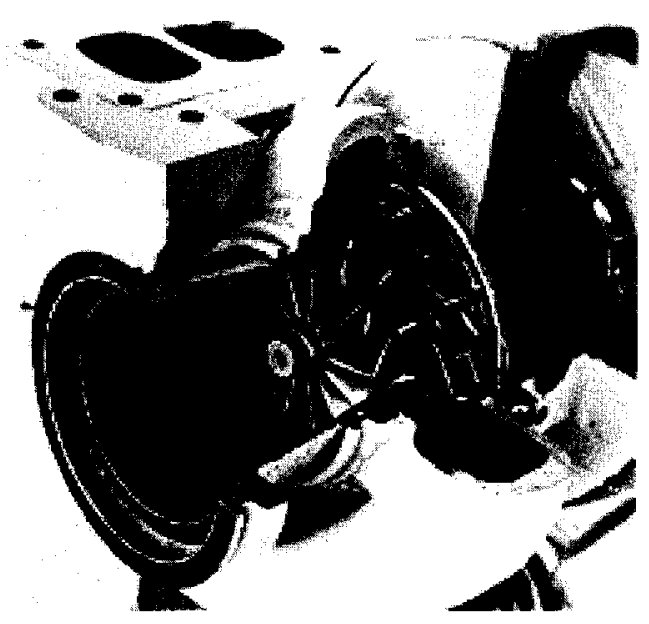

(b)

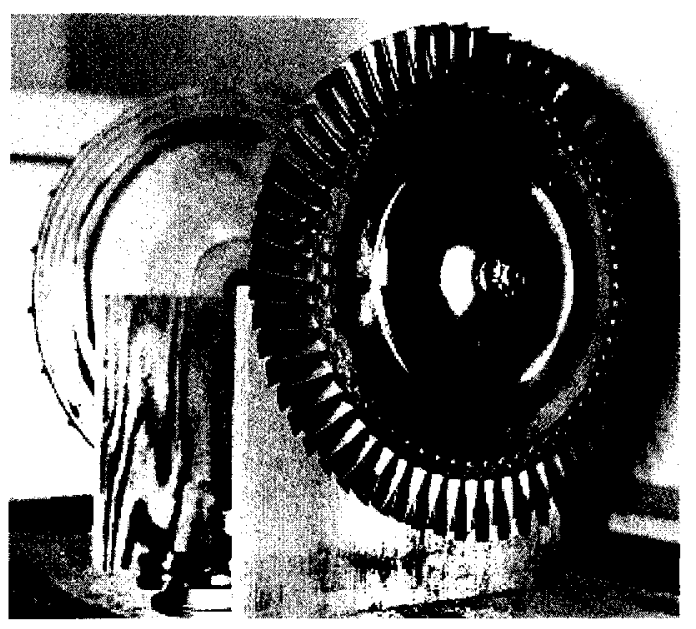

Figure 1.3: (a) Radial inflow turbine and (b) axial flow turbine (Moustapha et al, 2003)

A typical turbine stage is usually divided into 2 or 3 components. The first component is the stationary blade row (nozzles) or volute, which generates the initial 
swirl components and the high kinetic energy which will be extracted by the rotor. The rotor, of either axial or radial configuration, in turn converts the high velocity, high pressure flow into shaft power. In some cases, a diffuser is also required to recover some of the kinetic energy of the flow leaving the rotor, Japikse and Baines (1997).

Though the focus of the thesis is on the development of a comprehensive design tool for the preliminary design of axial flow turbines, significant computational fluid dynamics (CFD) was used in evaluating the design. Additionally, the components have also been assessed by finite element analysis (FEA) to obtain preliminary stress estimates and an approximation for fatigue and creep life. For both analyses, commercial codes CFX-5 and ANSYS 9.0 were used.

To achieve the goals of the turbine design, several tasks were outlined at the onset of the project. A summary of these tasks include:

- Literature review

- Development of design tools (theory)

- Verification

- Analysis and discussion

These tasks further provide the reader with an outline of the thesis. 


\section{Chapter 2}

\section{Literature and Theoretical Reviews}

A literature review including an overview of gas turbine turbomachinery design theory will be presented in this chapter. Design principles for the selection of various turbine configurations, design approaches, and loss systems for use in axial turbine application are presented. A discussion of the structural integrity will follow and include methods for blade stress estimation, as well as considerations for vibration and fatigue. Finally, a discussion on material selection and computational methods for fluid dynamics and structural analyses will be presented.

This chapter will only present an overview of the principles and theories applicable to the design of gas turbines. Any particular theories or methods used in the design process will be covered in detail in later chapters. 


\subsection{Turbomachinery Selection}

As previously mentioned, the purpose of the turbine is to extract the energy from the incoming fluid and transfer this energy into shaft power to drive the compressor, auxiliaries, fan or output shaft. As discussed by Japikse and Baines (1997), the fundamental equation for the specific work (power per unit mass flow, $\mathrm{w}_{\mathrm{sp}}$ ) delivered to the flow is the Euler turbomachinery equation given by Equation 2.1.

$$
\mathrm{w}_{\mathrm{sp}}=\mathrm{U}_{3} \cdot \mathrm{C}_{\theta 3}-\mathrm{U}_{2} \cdot \mathrm{C}_{\theta 2}
$$

where $\mathrm{U}$ is defined as the blade speed, and $\mathrm{C}_{\theta}$ is the tangential component of velocity at that particular station.

For an axial flow machine, the inlet and outlet blade speed is nearly constant, and the majority of the work extraction is due to the change in flow angle. In the case of a radial machine, the inlet radius is much larger than the outlet; thus it contributes largely to the work output of the stage. Therefore, the designer can see that for a given pressure ratio the radial machine is preferred in applications which require larger specific work; whereas, the axial machine is preferred in applications which require larger flow levels, Japikse and Baines (1997). For preliminary design studies and initial decisions regarding the type of turbomachine, various non-dimensional parameters are required. Nondimensional parameters such as stage loading or work coefficient, $\psi=\Delta \mathrm{h}_{0} / \mathrm{U}^{2}$, as well as flow coefficient, $\varphi=\mathrm{C}_{\mathrm{x}} / \mathrm{U}$, and specific speed

$$
\mathrm{N}_{\mathrm{S}}=\frac{\omega \mathrm{Q}^{1 / 2}}{\Delta \mathrm{h}_{0}{ }^{3 / 4}}
$$

play a major role early in the design. In the above equations, $\Delta \mathrm{h}_{0}$ represents the change in enthalpy required to produce the required work output, and $\omega$ is defined as the rotational speed in rad/s. Q is defined as the volume flow rate of the given gas. For 
turbines, radial and axial machines are quite different and are typically used for distinct applications. A brief explanation of each will be given.

The radial turbine is typically found in applications which are driven by high pressure ratio per stage (in excess of about 3:1) and low flow rates (typically below 2-3 $\mathrm{kg} / \mathrm{s}$ ). Although the efficiency of the radial turbine is conventionally not as high as their axial counterparts, they still appear in applications such as microturbines and turbochargers. A plot of radial turbine efficiency against specific speed is shown in Figure 2.1.

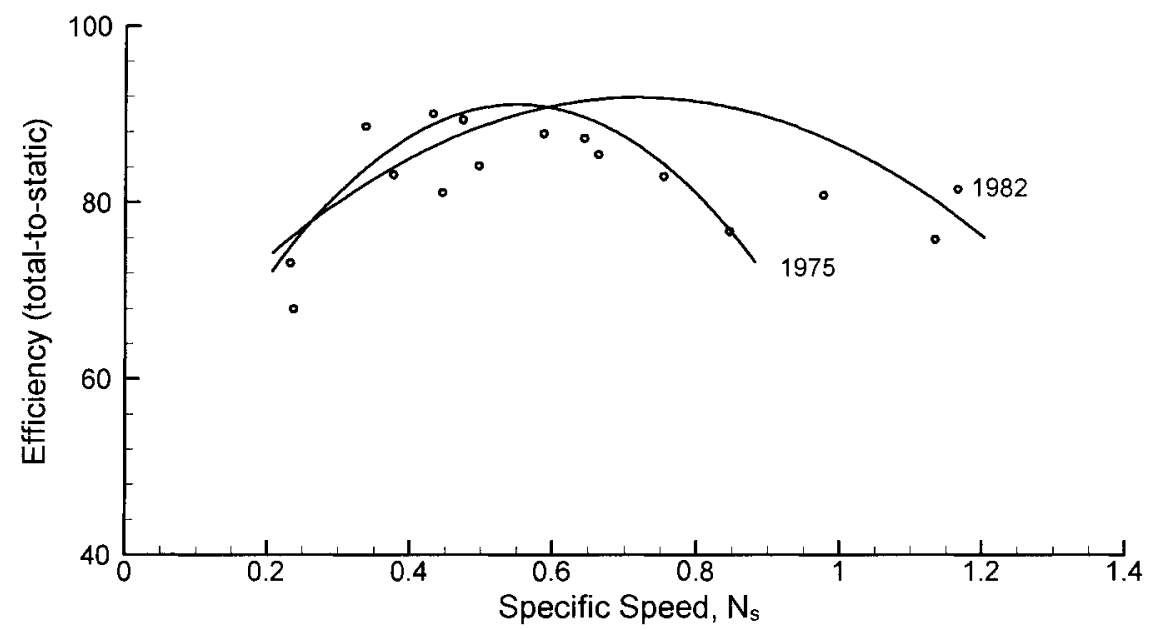

Figure 2.1: Efficiency versus specific speed for radial turbines (adapted from Japikse and Baines, 1997)

It can be seen how developments in turbine design have extended the range of peak efficiency particularly at high specific speeds over the years, Japikse and Baines (1997). The efficiencies of some 40 radial turbine stages are plotted in terms of stage loading and flow coefficients in Figure 2.2, Chen and Baines (1994). This figure highlights the combination of stage loading and flow coefficient required for high efficiency in this class of turbine. Data points and contours in Figure 2.2 show total-tostatic isentropic efficiency. The stage loading and flow coefficients for radial turbines differ slightly to that presented earlier. In Figure 2.2 the stage loading coefficient is defined as $\psi=\mathrm{C}_{\theta 2} / \mathrm{U}_{2}$, where 2 indicates the rotor inlet conditions. Likewise, the flow 
coefficient is defined as $\varphi=\mathrm{C}_{\mathrm{x} 3} / \mathrm{U}_{2}$, where $\mathrm{C}_{\mathrm{x} 3}$ is defined as the axial component of velocity at the outlet of the rotor.

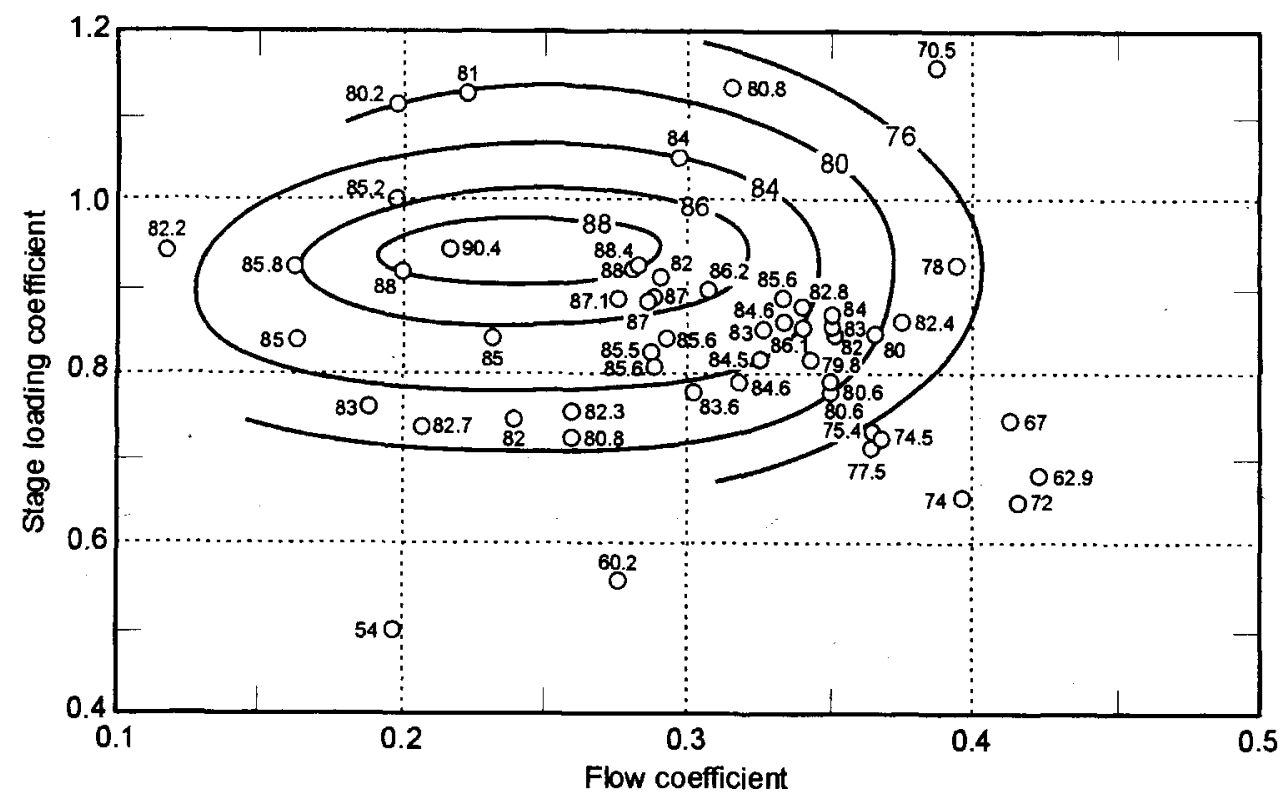

Figure 2.2: Stage loading and flow coefficient for radial turbines (Chen and Baines, 1994)

In an axial turbine, the objective is to achieve the work extraction through changes in flow angle. This provides the capability of producing high levels of work with high mass flows, but there is a greater restriction on the pressure ratio that can be handled in a given stage, Japikse and Baines (1997). Current trends in axial turbines are in the development of highly loaded blade rows for further advances in blade number and weight reduction.

In the initial selection and sizing of machine type, it is typical to look at specific speed correlations. Balje (1981) presents a plot of maximum turbine efficiencies as a function of specific speed. This is shown in Figure 2.3. Here, the total-to-static efficiencies of various turbine configurations are presented. 


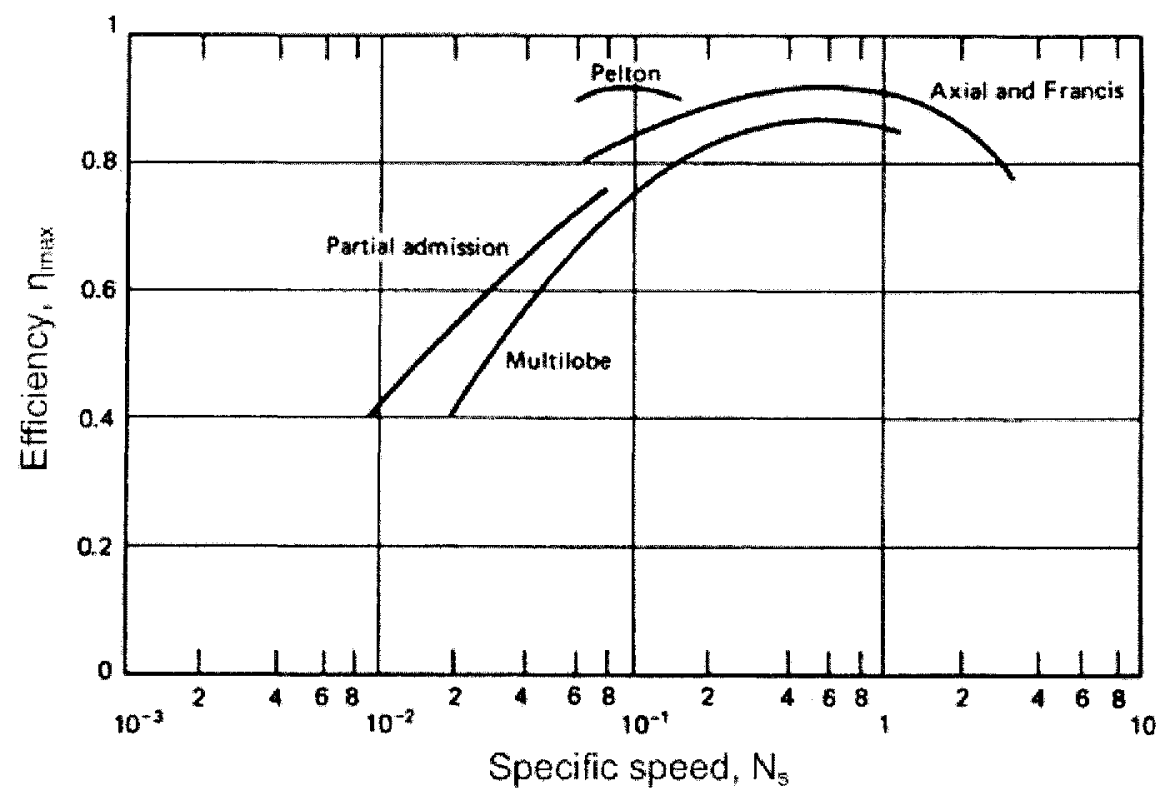

Figure 2.3: Maximum turbine efficiency as a function of specific speed (Balje, 1981)

The specific speed does not give any indication of the size of the machine. It has been shown by Moustapha et al (2003) that the specific diameter correlates well with specific speed. This can be seen in Figure 2.4, where it is plotted for a range of radial turbines at the best efficiency points. From the specific diameter, an early indication of overall component size may be estimated in advance of any detailed design.

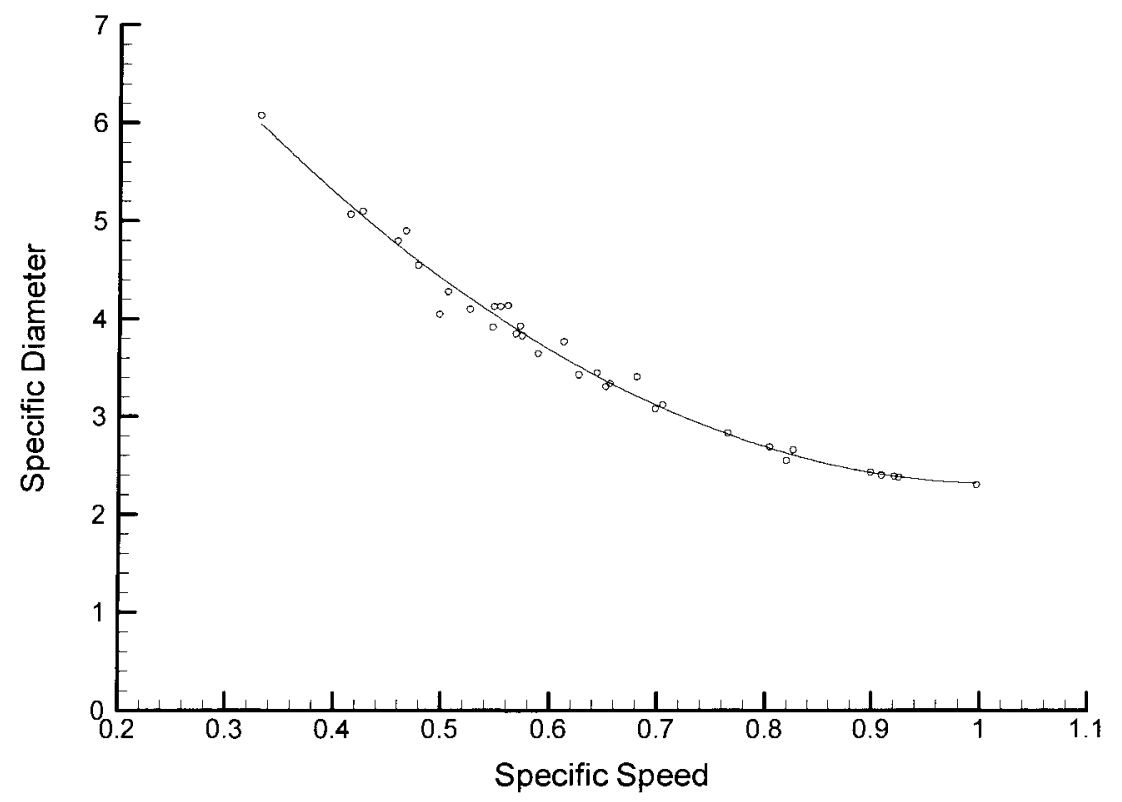

Figure 2.4: Correlated specific speed and specific diameter for radial turbines for maximum efficiency (Moustapha et al, 2003) 


\subsection{Design Approach}

The first step in the design of a new engine is the selection of the overall cycle configuration. This is normally specified by an engine performance group. Once this is completed, the preliminary design of the turbine stages may begin. At this point, the turbine expansion ratio, power requirement, and turbine inlet conditions are known.

With these specifications set, a proper turbine configuration may be selected as outlined in the previous section. The next step is to perform the preliminary meanline design, typically conducted at 50 percent blade span (blade height). In order to familiarize the reader, Figure 2.5 presents various turbomachinery terminology and nomenclature.

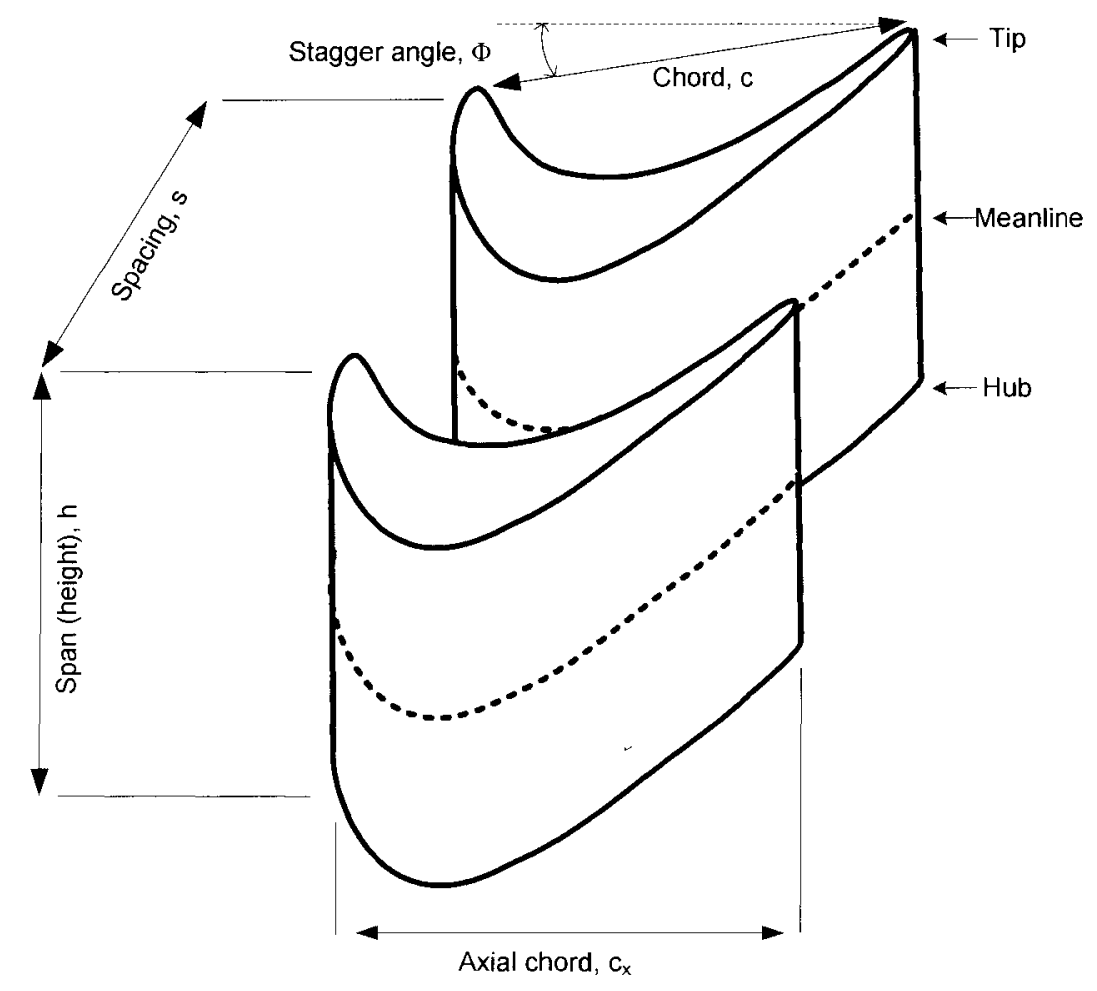

Figure 2.5: Blading terminology

Variations of the meanline design method exist and may include: use of simple stage correlations and basic conservation principles, use of fundamental physical models or simply scaling an existing design. Stage correlations for axial turbines such as the 
Smith chart, shown in Figure 2.6, are used to select suitable values of stage loading and flow coefficient. In this instance, the stage loading and flow coefficients are defined as $\psi=\Delta \mathrm{h}_{0} / \mathrm{U}^{2}$ and $\varphi=\mathrm{C}_{\mathrm{x}} / \mathrm{U}$, respectively. In this case, inlet or exit conditions may be used as they do not differ appreciably in axial machines. By also selecting the stage reaction and the mean blade speed, the velocity triangles and thermodynamic state of the working fluid may be calculated at the inlet and exit to each blade row.

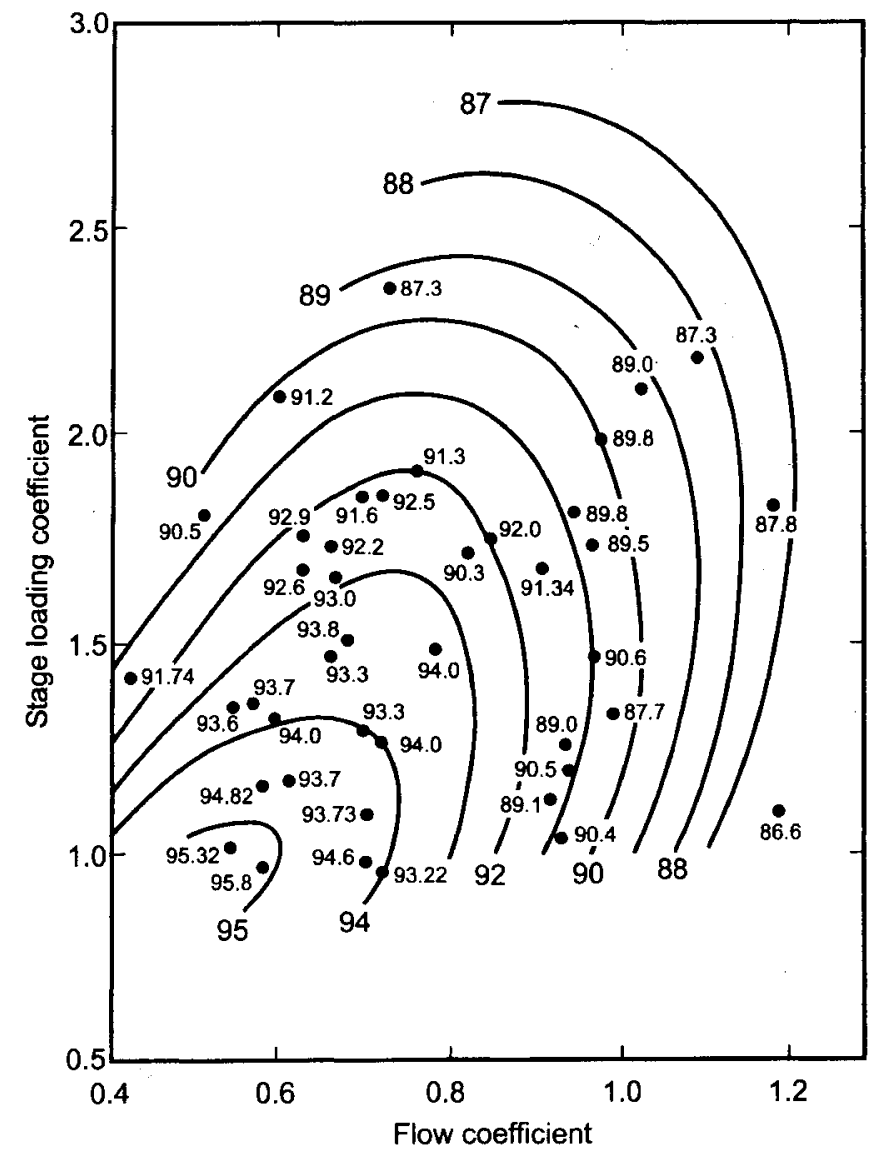

Figure 2.6: Smith chart for axial turbine stage efficiency (adapted from Smith 1965)

The efficiency values shown in Figure 2.6 have been adjusted to exclude tip clearance loss, and therefore are higher than would be expected for similar turbines in actual operation. Due to the advances in gas turbine technology, it is advised that this figure be used only as a selection guide for preliminary design studies. 
By including additional parameters such as solidity, lift coefficient, and aspect ratio, the meanline design can also give an estimation of overall size, blade span and blade number. These will be discussed in detail later. The next step in the design process is typically the through-flow design. Here, a radial dimension is added for the flow to be analyzed on a two-dimensional plane from hub to tip. Following this, the airfoil design may begin. The design of the airfoil includes specifying and evaluating the twodimensional blade profiles, the way the profiles are stacked, and the full threedimensional shape of the blade. A summary of the design process is given in Figure 2.7. A detailed flow chart of the design tool's functionality is presented later.

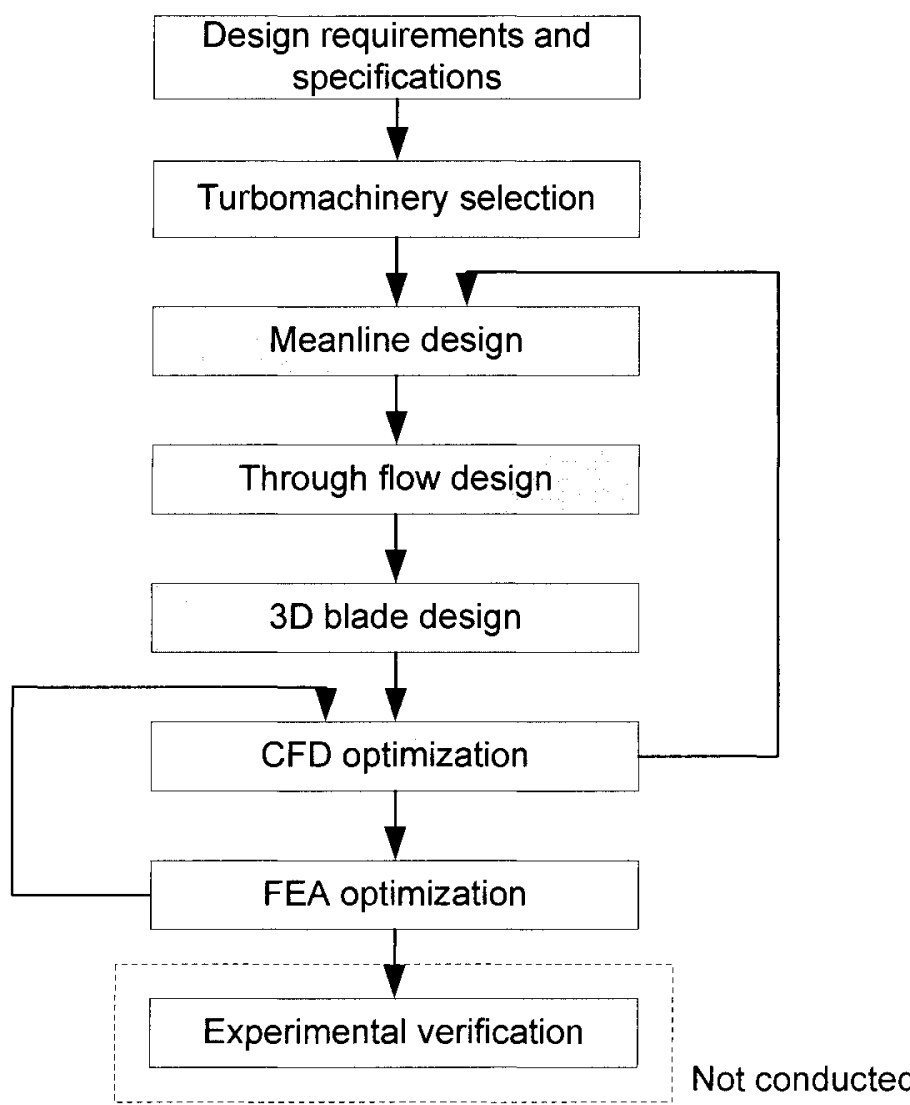

Figure 2.7: Design process summary

It should be stressed that this design process, like most others, is iterative in nature and is by no means a linear process. Early design decisions will be reviewed, revised and reconsidered many times throughout the course of the design. In addition to 
the aerodynamic design and performance evaluation, the turbine designer must also bear in mind structural considerations and review these thoroughly before the design is complete.

\subsubsection{Preliminary Meanline Design}

The preliminary meanline design process typically begins with the selection of stage loading and flow coefficient. In addition, it is also necessary to select an appropriate degree of reaction and rotational speed. It is important to note the nature of the turbine design since the selection of these parameters may be dependant on other engine components. For instance, the selection of a gas generator speed is usually limited by the compressor which is being driven. For the turbine designer, this is less than ideal. From the Euler Equation, it can be seen that a reduction in blade speed reduces the amount of work that can be produced for a given stage loading. Therefore, the turbine designer will have to consider additional stages or choose a higher stage loading. This has led to the development of highly loaded turbine stages to reduce cost, weight and number of components.

Several researchers have published methods for attaining highly efficient turbine stages at significantly high stage loadings. Selection of a high stage loading requires careful control of the flow development within the stage. These publications are discussed later.

Byerley et al (2004) describe a method that uses a systematic and graphical approach through the preliminary design process of rotating machinery. They describe a method of arriving at a design envelope based on a correct combination of design variables.

Vazquez et al (2003) discuss the current technology limits that are exceeded by high stage loading turbines and the special aerodynamic and geometrical features that come up. They discuss approaches used to reduce the number of stages and blades by 
highly loading low pressure turbines. According to the authors, most modern direct drive low-pressure turbines have been designed for a moderate stage loading of $\psi \sim 2$. By further increasing the loading to $\psi \sim 3$, they were able to reduce LP turbine weight and cost by approximately 20 percent. It is further stated that the aerodynamic characteristics of these turbines lie well beyond the current levels of technology, and that estimating the efficiency reduction that comes with the added loading, may prove difficult as the classical efficiency correlations need to be extrapolated. References are made to numerous test beds and research programs being conducted, and a new proposal for an efficiency chart is made.

Another important design criterion is the choice of reaction. This defines how the complete stage expansion process is split between the rotor and stator, where a high reaction signifies large acceleration in the rotor. Typical values of reaction are approximately $0.4-0.5$ as these generally lead to the highest turbine stage efficiencies, Wilson and Korakianitis (1998). Higher reaction designs lead to reductions in the relative total temperature, and thus the blade metal temperature of the rotor, which may increase the blade life. However, at the same time, a high reaction also indicates a high level of exit swirl, which may lead to less efficiency due to losses in the duct. Choice of reaction further influences off-design efficiency, blade spacing and platform fit, as well as bearing loads. Further information can be found in Moustapha et al (2003).

The three dimensionless parameters just mentioned (stage loading, flow coefficient and reaction) may be used in setting up the velocity triangles. Their effects on the predicted efficiency are evident from the Smith chart, shown previously in Figure 2.6, and also discussed by Saravanamuttoo et al (2001). Low values of stage loading and flow coefficient lead to the best stage efficiencies due to the low gas velocities and reduced friction losses. This, however, implies more stages are needed, leading to increased cost and weight. Tradeoffs must be considered in deciding whether the efficiency penalty is worth the reduction of overall weight and cost of the engine. 


\subsubsection{Through Flow Design}

The through flow design process provides an extension to the meanline design by calculating the flow velocity, state, and direction from hub to tip. At this stage, the axial and radial variations in the flow are considered. Modern turbine blades are extremely complex three-dimensional shapes. This is required due to the radial variation in blade speed and the desire to limit secondary flow losses. The change in blade angle across the blade span can be expressed by either a free-vortex or forced-vortex design. Vortex theory described in Saravanamuttoo et al (2001) shows that if the elements of the fluid are to be in radial equilibrium, an increase in static pressure from hub to tip is necessary whenever there is a swirl component of velocity. This can be seen in Figure 2.8.

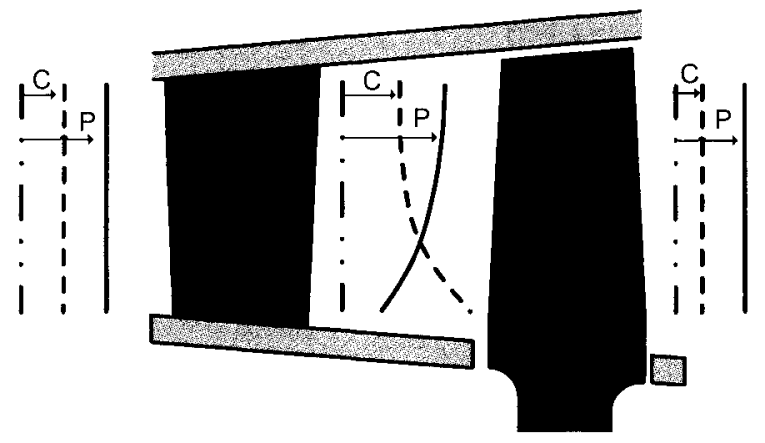

Figure 2.8: Velocity and pressure distribution for free-vortex design

Although it is not essential to design for free vortex flow, it is still done in many turbine stages. Free-vortex design requires that the stagnation enthalpy and axial velocity must be constant over the annulus, and that the swirl velocity is inversely proportional to the radius. Using this method, the velocity triangles at the root and tip may be constructed by simply setting

$$
\mathrm{C}_{\theta} \cdot \mathbf{r}=\text { constant }
$$

and determining the flow angles and velocity components. In this instance $r$ is defined as the local blade radius. At this time, the designer must also check that the reaction and Mach numbers at each location from root to tip are suitable. Experimental results show 
that large reaction at the blade tip leads to excessive tip leakage losses, and that low reaction at the hub may cause flow separation in this region, also resulting in large losses, Sawyer (1985). In a forced-vortex design, the designer is able to specify the flow angles at the outlets of the blades, or aim to have constant mass flow per unit area at all radii. Yet according to Saravanamuttoo et al (2001), "for turbine of modest radius ratio, it is very doubtful indeed whether such refinements are more than an academic exercise".

At this point, assumptions of the distributions of aerodynamic blockage, deviation and loss are also needed. These may be estimated from the meanline design. It is important to note that these will not be constant across the span and should be checked again once the airfoil design is complete. Typically, loss buckets will be constructed for the rotor and stator. The low loss that occupies the middle of the span consists mainly of profile loss as secondary flow losses dominate the hub and tip regions due to the endwall boundary layers.

\subsubsection{Airfoil Design}

In the design of the airfoil, it is crucial that the resulting blade angles match the relative inlet and exit flow angles and expand the gas with minimum losses. To do this effectively, consideration of incidence and deviation must be included. This section will concentrate on the aerodynamic design, with structural and vibrational considerations examined later.

With the velocity triangles specified, the designer must choose a value for the design incidence. Various correlations exist for determining the incidence (i) in turbine blades. One empirical correlation from data of Moustapha et al (2003) is given by

$$
\mathrm{i}=0.175 \cdot \alpha_{\text {in }}-5.5
$$

and from Dunavant and Erwin (1956) 


$$
\mathrm{i}=14 \cdot\left(1-\frac{\alpha_{\text {in }}}{70}\right)+9 \cdot\left(1.8-\frac{\mathrm{c}}{\mathrm{s}}\right)
$$

where $\alpha_{\text {in }}$ is defined as the inlet blade angle, $\mathrm{c}$ is the true chord, and $\mathrm{s}$ is the blade spacing. These correlations lead to significantly different values for design incidence for a given inlet angle. However, according to Wilson and Korakianitis (1998), this is really not of great significance as turbine blades are often designed without the incidence being incorporated. In examining Figure 2.9, one can clearly see that the profile loss coefficient for reaction blades is quite flat for incidences ranging from -15 to 15 degrees.

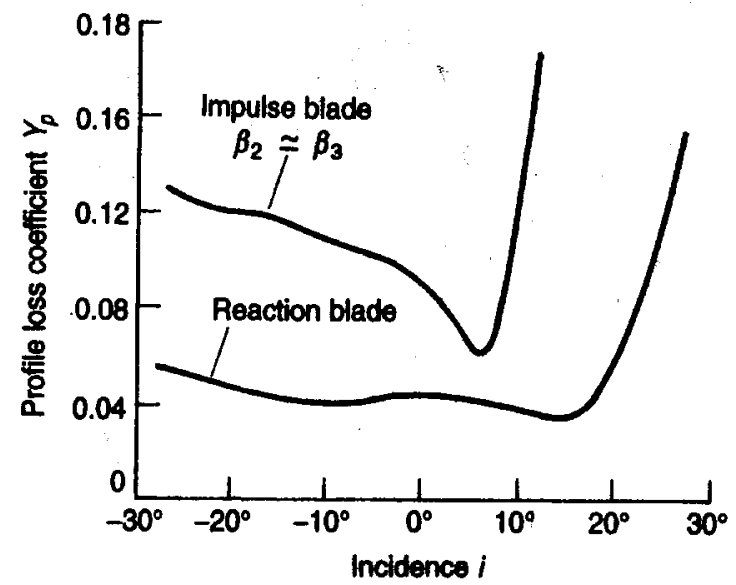

Figure 2.9: Effect of incidence on profile loss (Saravanamutto et al, 2001)

It should be noted however, that the incidence will also change during off design operation, and therefore, care should be taken in selecting the amount of incidence at the design point. These correlations are used to give the designer a starting point in the preliminary design.

Likewise, differences in outlet flow and metal angle must also be considered. This is known as deviation. Unlike incidence, deviation may play a significant role in the performance of the blade row. If the flow is under turned, the blade row will produce a lower change in tangential velocity, and therefore a lower torque, work output and enthalpy drop. Similar to incidence, many correlations exist for predicting deviation in the initial stage of design. Wilson and Korakianitis (1998) recommend the usage of a 
method outlined by Ainley and Mathieson (1951) as it seemed to provide the most accurate and reasonable of the alternatives and it is based on blade opening rather than cascade data.

Next, the design must quantify the two-dimensional profile of the airfoil. The two-dimensional profile not only affects the performance of the meanline analysis, but may also have an effect on the secondary loss. The influence of profile shape is discussed by Haller (1997), as well as many other researchers. It has been found that 'aft-loaded' profiles generate significantly lower losses than fore-loaded or flat-topped designs, particularly at high Reynolds numbers ( $>2 \times 10^{5}$ based on chord) and turbulence levels ( $>4$ percent).

By evaluating the airfoils on blade-to-blade surfaces, pressure distributions and Mach number contours can be used to assess the performance of the design. Next, the airfoils are stacked in a manner to limit secondary and tip losses without adding considerably to the bending stresses. When the full three-dimensional blade has been generated, it may be subjected to channel flow analysis using computational fluid dynamics. Various methods exist in literature for designing turbine blade sections. Moustapha et al (2003) presents a method of using Bezier polynomials. This gives the designer a great deal of flexibility while maintaining a high degree of continuity of the curve and its derivatives. In addition, Wilson and Korakinanitis (1998) present alternative methods of blade design based on various direct and inverse methods. Modern computer codes and CFD programs offer blade generating modules, which prepare complex blade patterns and shapes for use in determining structural and aerodynamic performance. Later sections will discuss the use of Bladegen, BladegenPlus, and CFX-5 for designing blades and screening the preliminary designs. Bladegen and BladegenPlus are commercial software packages used for designing and analyzing complete three-dimensional turbomachinery blade designs. Bladegen is simply a geometry modeler where the BladegenPlus module is used for obtaining threedimensional viscous solutions of the models. CFX-5 is a widely used, commercial 
software package used for obtaining detailed CFD solutions for various research and industrial applications.

As stated previously, careful attention must be paid in the design of efficient highly loaded turbine blades. Corriveau and Sjolander (2004) suggest that to succeed in designing such optimized blades, it is necessary to determine the maximum allowable amount of diffusion on the blade surface. The diffusion limit is imposed by the need to avoid separation of the blade boundary layer which is associated with high losses, especially if the separated flow does not reattach. Haller and Anderson (2002) describe a new approach for the preliminary design of the stage, adapted from the Denton $\mathrm{U}^{3}$ method. In this method, standard empirical loss correlations are abandoned, and the blade velocity distribution is optimized for minimum profile and secondary loss. They further investigate the advantages of 'controlled flow' by skewing the sections closed towards the endwalls and opening the throat at mid-span to keep the same overall throat area. This reduces secondary losses near the endwalls and preserves the mean reaction.

Further performance gains can be attributed to reduction of secondary losses in the blade passage. The differences in straight and compound lean are demonstrated by Haller (1997). Conventionally, secondary losses are a strong function of blade aspect ratio, or height-to-chord ratio. According to Saravanamuttoo et al (2001), a value of h/c between 3 and 4 would be very satisfactory, and it would be unwise to use a value below 1. In evaluating their performance prediction methods, Kacker and Okapuu (1982), publish the values of stage loading, flow coefficient, pressure ratio, aspect ratio, and turbine efficiency for 33 known turbines. It is found that values of aspect ratio range anywhere from 0.25 to 5.5 , depending on the number of stages and application.

An estimate of blade count is conducted through the addition of a solidity parameter. To do this, a value of solidity is usually chosen based on empirical rules outlined by previous designers. Two well known methods of selecting solidity of turbine blades are that of Ainley and Mathieson (1951) and Zweifel (1945). Zweifel observed 
that the losses from turbine blades were minimal at a particular tangential force coefficient $\left(\mathrm{C}_{\mathrm{L}}\right)$ defined as:

$$
C_{L}=2 \cdot\left(\frac{s}{c_{x}}\right) \cdot \cos ^{2}\left(\alpha_{e x}\right) \cdot\left|\tan \left(\alpha_{\text {in }}\right)-\tan \left(\alpha_{e x}\right)\right|
$$

where $c_{x}$ is defined as the axial chord component and $\alpha_{\mathrm{ex}}$ is given as the exit flow angle.

Historically, an empirical rule was to design for a Zweifel coefficient of about 0.75-0.8, but this can be increased with extensive use of CFD methods to optimize the blade loading distribution. Today's designers are able to achieve Zweifel loadings of about 1.1 .

Ainley's method of optimum solidity is based on curve-fitted data obtained from the loss coefficients provided by Ainley and Mathieson (1951). Figure 2.10 summarizes the results.

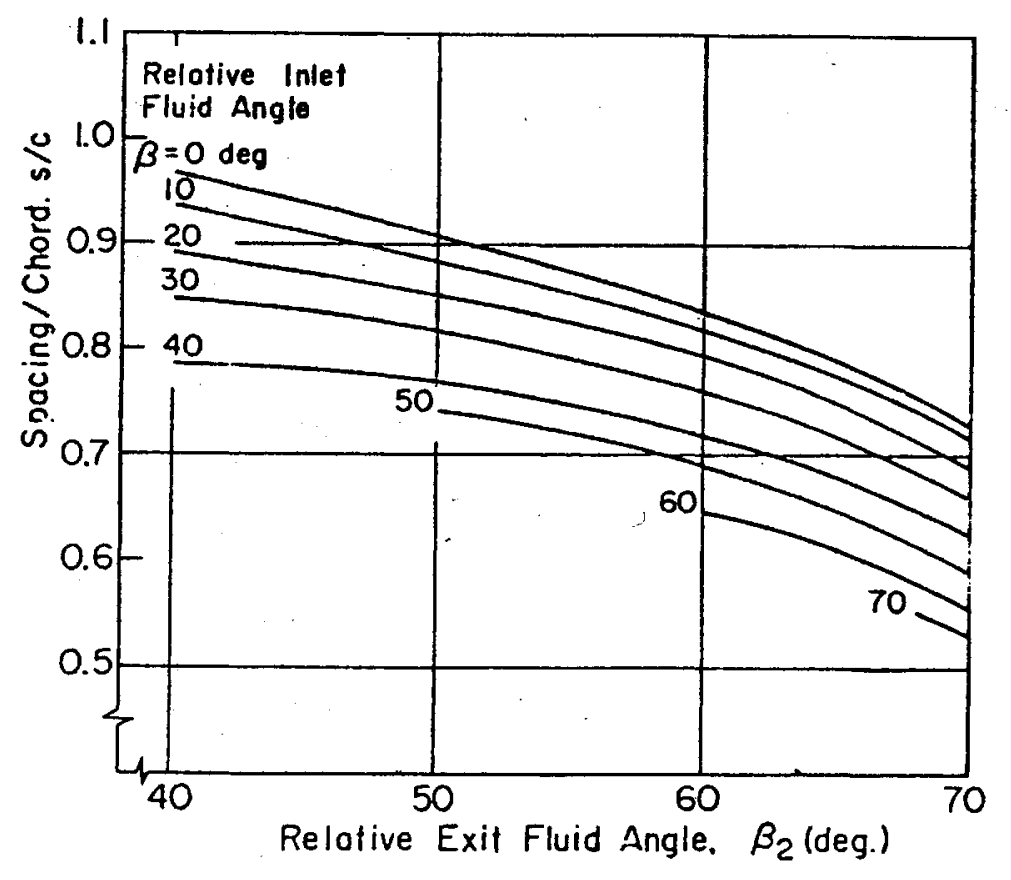

Figure 2.10: Optimum blade spacing, Ainley's correlation (Sawyer, 1985) 


\subsection{Turbomachinery Losses and Performance Evaluation}

Numerous methods of performance prediction exist in literature for axial turbines. The best known and documented are those of Ainley and Mathieson (1951), Dunham and Came (1970), and Kacker and Okapuu (1982). Each of these loss systems are an addition or modification to the last, with the most recent tested against known efficiencies of 33 turbines of recent design. These methods are empirically based and valid design point prediction only. A somewhat different approach, although still based on empirical cascade data, is given by Craig and Cox (1971). This method attempts to take into account the full range of Reynolds numbers and aspect ratios encountered in steam and gas turbines. Additionally, this method attempts to deal with most of the auxiliary sources of loss which are sometimes omitted from other published methods. Authors such as Smith (1965) and Balje (1981) present methods which base turbine stage performance on overall parameters such as work coefficient and flow coefficient. These methods are most useful in the preliminary design stage where the details of the design are still unknown. A summary of various loss mechanisms and their corresponding performance prediction methods are given by Sieverding (1985a).

An understanding of the physical origin of turbomachinery losses should occur before any discussion of loss system. These losses may be grouped into various categories such as boundary layer and trailing edge loss, tip leakage loss, endwall loss,

effects of heat transfer, and miscellaneous losses. Although these losses may be discussed independently, it should be stressed that the loss mechanisms are seldom independent. Typically, loss coefficients are defined in terms of stagnation pressure or kinetic energy losses. According to Denton (1993), a more rational measure of loss in an adiabatic machine is entropy creation. Entropy may be created by viscous friction effects in either boundary layers or free shear layers. Additional sources include mixing processes, heat transfer and non equilibrium processes such as shock waves. 


\subsubsection{Profile Losses}

The first class of losses to be discussed is profile losses. Profiles losses are those due to skin friction on the blade surface and are strongly dependant on the blade area in contact with the fluid, surface finish, Reynolds number and Mach number. These losses are governed largely by blade geometry and are a strong function of the blade boundary layer. Surface roughness effects are presented by Hummel et al (2004), where total pressure losses are evaluated for various surface roughnesses within a high-speed cascade wind tunnel. Since the velocity changes most rapidly near the surface of the blade, it is evident that most of the entropy generation is concentrated in the inner part of the boundary layer. In fact, Dawes (1990) gives a more detailed breakdown of the entropy generation in the boundary layer, showing that about 90 percent of the entropy generation occurs within the inner part of the layer. Roelke and Haas (1983) investigate the effects of rotor blade thickness and surface finish, of as-received cast rotor blades, on the performance of a small axial flow turbine.

\subsubsection{Secondary Losses}

Another form of loss occurs due to the presence of secondary flows. A detailed description of secondary flow vortex structures and their effect on endwall boundary layer characteristics is given by Sieverding (1985b). Secondary flows arise due to the interaction of the endwall boundary layer and the flow turning of the passage which cause some parts of the fluid to move in directions other than the principal direction of flow. This action creates two contra-rotating vortices as the endwall boundary layers roll up under the action of the cross passage pressure gradient created by the passage curvature as shown in Figure 2.11. The vortices then distribute lower momentum fluid of the endwall and blade boundary layers into the main stream acting as a source of loss. Endwall flow features, as well as experimental and numerical studies on turbine stage aerodynamic loss generation, are provided by Gallus et al (1994), along with Ho and Lakshminarayana (1996). Another contribution to the overall loss within a turbine stage occurs as a result of the blade trailing edge thickness. In this case, a momentum deficit 
exists at the trailing edge where the two boundary layers from the suction and pressure surface meet. The velocity deficit in the wake continues to decay downstream, and as a result of the mixing process, incurs another source of loss. According to Denton (1993), we do not need to know the exact rate of decay to predict the overall result. As long as the mixing is complete by the time the flow leaves the region of interest, the total entropy created can be calculated without knowing the details of how and where the mixing takes place. Additionally, an experimental investigation of an axial flow turbine rotor was conducted by Ristic et al (1999) to determine the three-dimensional flow field downstream of the rotor. The wake deficit was found to be substantial, particularly at 1 percent chord, downstream of the rotor. Furthermore, radial velocities in the wake and endwall region were also found to be substantial, and two counter-rotating secondary flows are identified in the blade passage.

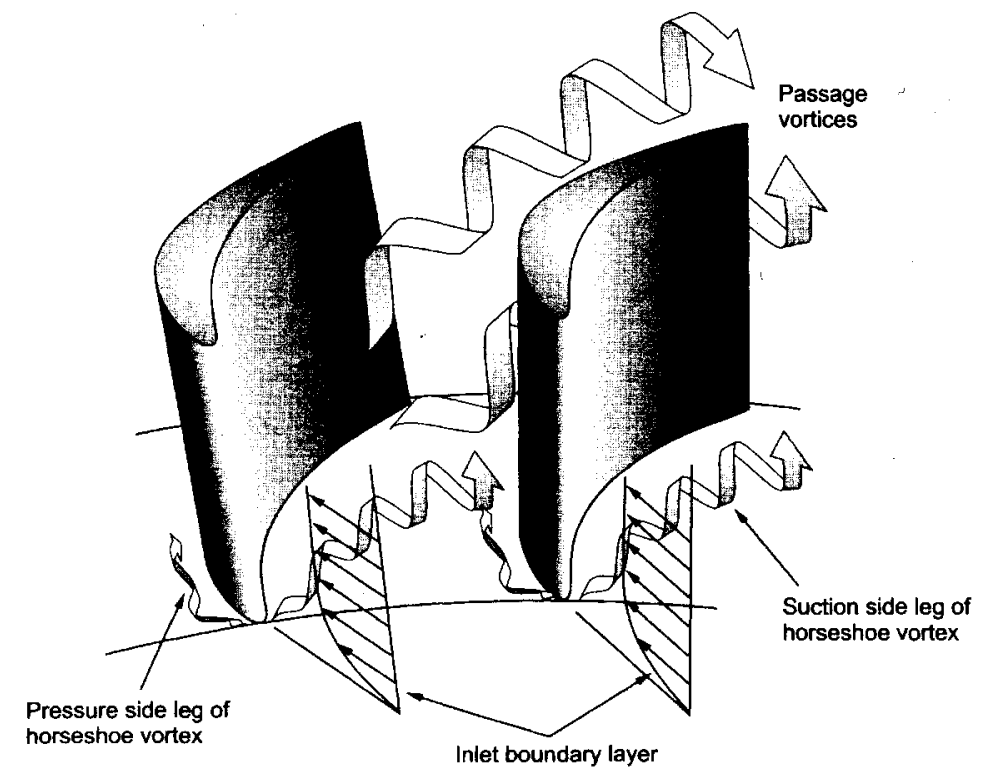

Figure 2.11: Schematic diagram of passage vortex formation (Moustapha et al, 2003) 


\subsubsection{Tip Clearance Losses}

Lastly, the effects of tip clearance in axial turbine rotors have been presented by numerous researchers. In an unshrouded blade passage, fluid leaks from the pressure side to the suction side of the rotor blade contributing little or no expansion work. In addition, a tip leakage vortex is generated by the differential shear of the moving blade and the stationary casing. The vortex then interacts with other passage vortices producing very complex flow patterns. The situation for shrouded blades is somewhat different as fluid now leaks from leading edge to trailing edge. This leakage flow however, can be limited by the addition of sealing fins or fences. As a result, a 1 percent increase in clearance/span ratio results in about a 2 percent loss for an unshrouded turbine and only 1 percent for a shrouded turbine, Figure 2.12, Moustapha et al (2003).

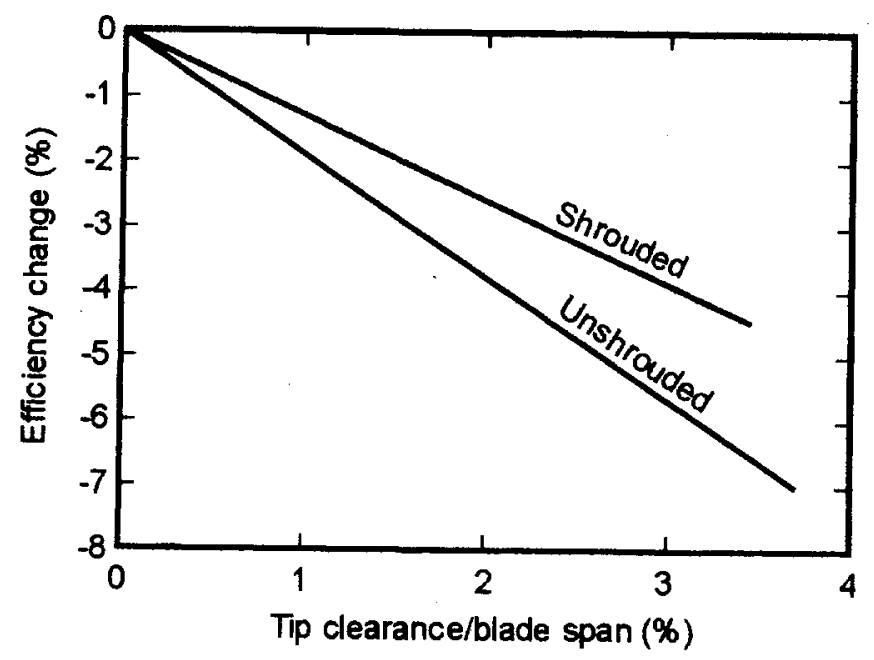

Figure 2.12: Efficiency change with clearance (Moustapha et al, 2003)

A detailed literature review on the studies of tip clearance flows in axial turbines has been made available by Sjolander (1997). According to Graham (1986), blade unloading is a major feature of the pressure field near the blade tip and is strongly dependant on the tip clearance height, especially when reduced below 1.5 percent span. A more detailed measurement of blade loading is outlined by Sjolander and Amrud (1987). It was found that the local blade loading actually increases as the tip is approached, due primarily to a strong suction peak induced by the tip vortex on the 
suction surface. Further investigations were performed by Yaras and Sjolander (1992) to investigate the effect of simulated rotation. Tip clearance effects in a turbine rotor were also investigated by Xiao et al (2001a). They found that the highest pressure drop and the highest total pressure loss were both observed in the region of the tip leakage vortex, where the loss is nearly twice as high as that near the passage vortex region. However, the loss region is considerably wider in the passage vortex region and thus contributes to a larger portion of the total losses. The second part of the paper by Xiao et al (2001b) further interpolates the vorticity, velocity, and turbulence fields at several axial locations. 


\subsection{Structural Integrity}

In addition to aerodynamic design and performance, considerations must also be made for the structural integrity and mechanical design of the turbine components. Material and manufacturing limitations will have a direct effect on the turbine geometry and its operating conditions. A distinction must be made between steady and unsteady stress states. Steady-state stresses, such as centrifugal loading, pressure loading, and thermal stresses typically cause creep failure in plastic deformation. Unsteady stresses, arise from the interaction of rotating and stationary components, as well as any form of unbalance or natural vibration modes, which may cause fatigue failure due to crack initiation and growth.

\subsubsection{Stress Estimation}

Numerous textbooks present guidelines for turbine disk and blade stress estimation. One of the most practical methods for preliminary design expresses turbine blade stress as a function of $\mathrm{AN}^{2}$, where $\mathrm{A}$ is the annulus area and $\mathrm{N}$ is the rotational speed. Limits are then imposed for various design applications. Explanation of this method can be found in Saywer (1985), and Moustapha et al (2003). Although this limit clearly depends on material selection, blade taper and metal temperature, it will still aid the designer in determining the feasibility of the aerodynamic design during the preliminary design stage. If stress levels appear too high, one may choose to decrease the annulus area; however, this will lead to a decrease in blade aspect ratio thereby increasing Mach numbers and secondary flows. On the other hand, one may wish to reduce the rotational speed. This will however, lead to a higher stage loading and perhaps lower the engine efficiency. These examples clearly illustrate the tradeoffs that must be made between the structural and aerodynamic design. In addition to the $\mathrm{AN}^{2}$ relation, guidelines on the maximum permissible blade tip speed are imposed during the aerodynamic design. Depending on the application, limits are outlined by Logan (2003), along with Japikse and Baines (1997). 
Calculation of blade root stresses and gas bending stresses are carried out by Sawyer (1985), as well as Saravanamuttoo et al (2001). Computer programs to calculate various blade stresses can be found in Fielding (1982). For manufacturing consideration, additional guidelines for hub-to-tip ratio, trailing edge thickness and blade angle are given by Logan (2003).

\subsubsection{Creep}

Due to the high temperatures experienced within a turbine stage, it is required to perform an estimation of the creep behaviour of the rotor. When temperatures reach within 30 to 60 percent of the material's melting point, movements of atoms in the material will begin to induce very slow deformation known as creep. Various mechanisms of creep such as dislocation glide and grain boundary sliding are described in literature, Mangonon (1999), and Sims et al (1987). An idealized creep deformation response over time is shown in Figure 2.13.

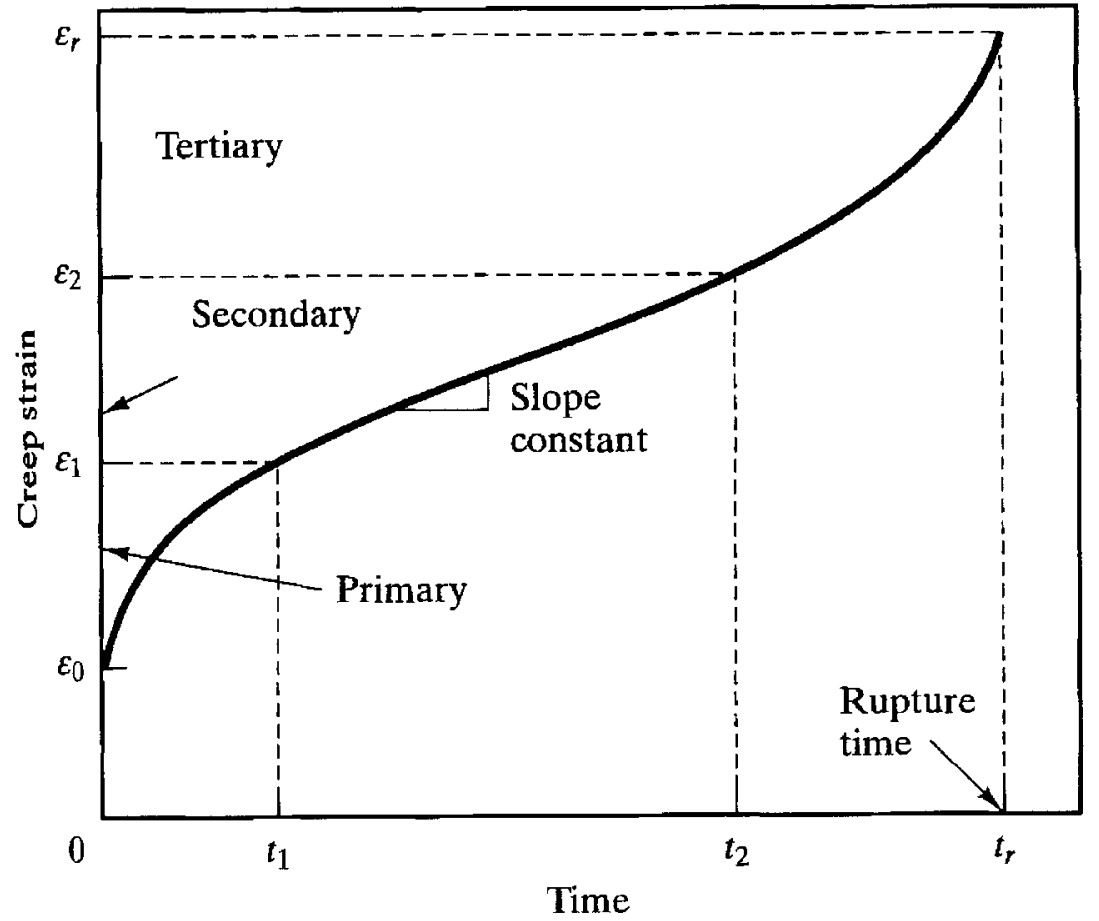

Figure 2.13: Schematic of creep-rupture showing stages (adapted from Mangonon, 1999), 
This figure clearly identifies the primary, secondary and tertiary regions of creep. Typically, the designer wants to ensure that the component exhibits creep within the secondary regime for the majority of its life. This allows the designer to more accurately predict the life of the component because of the linearity of the creep strain curve. Any component operating in the tertiary zone will be prone to an unstable creep strain rate and it could very likely fail without warning.

Creep testing can be time consuming and expensive; therefore, it is most often extrapolated from short-term tests. The most common method is employing the LarsenMiller parameter, Larsen and Miller (1952). It was proposed that for a given stress the time to rupture is related to temperature by the equation

$$
T(20+\log t)=\text { constant }
$$

where $\mathrm{T}$ is the absolute temperature and $\mathrm{t}$ is the time to rupture. According to Japikse and Baines (1997), the Larson-Miller parameter has been used extensively over the past 40 years for correlating short-term creep data with the prediction of long-term creep processes. Other sources suggest that the dominating creep mechanisms differ between short-term and long-term creep behaviour. Ashby (1972) presents the idea of deformation mechanism maps which display the fields of stress and temperature in which a particular mechanism of plastic flow is dominant. Manson and Haferd (1953) describe a different model required to account for the nonlinearity observed in the data when plotted as $\log t$ versus $1 / \mathrm{T}$ for constant stress.

A concise and effective review of the factors that must be considered in selecting, and designing turbine blade alloys, is presented by Huda (1995). Effects of crystal structure, solutes, precipitates, and grain structure are described. An example of creepfatigue failure of an aero-engine 2nd stage turbine blade is documented by Salam et al. (2002), indicating the combined effects of different failure mechanisms. 


\subsubsection{Vibration and Fatigue}

One of the most difficult failure modes to predict in gas turbine design is fatigue. Fatigue occurs as a result of repetitive loads which cause the part to fail abruptly without any prior permanent deformation. Typically, fatigue failure is grouped into two categories: High Cycle Fatigue (HCF) and Low Cycle Fatigue (LCF). HCF is categorized by phenomena relating to the blade passing frequency (the repetitive loading, or alternating stress, is typically of high frequency and low magnitude). It can usually be eliminated or avoided by design. LCF, on the other hand, deals with effects which occur at low frequency but typically higher stresses. These effects, occurring once per operating cycle cannot be eliminated, and effort must be made to minimize them. According to Rao (1991), blade failures due to fatigue are predominately vibration related. Whether high or low cycle fatigue, the designer must take into account all conditions to make an accurate prediction of the service life of the part.

Although various classical fatigue models exist, for example Manson (1966), they are based largely on low-strength, high-ductility metals. Care must be taken in evaluating modern engine components using these models as today's superalloys are mainly high-strength, low-ductility alloys. The work of Coffin and Schenectady (1954) presents a study of the effects of cyclic thermal stresses on a ductile material. The work focuses on the damage done to the structural material by high cyclic thermal stresses. Additionally, it quantifies the cycles to failure for a particular material. Arakere and Moroso (2001) discuss the fatigue failures in PWA1480/1493 single crystal nickel base superalloy turbine blades used in the space shuttle engine fuel turbopump. Crack initiation and growth due to HCF are discussed in depth and a detailed finite element model is presented.

Accurate prediction of component life requires that actual testing of component strain and temperature history be completed. For maximum benefit, the test component should be a laboratory specimen having similar geometry to the component of interest. The work of Ostergren (1976) has made significant effort in correlating uniaxial test data 
with real and idealized engine components. Prediction also depends on fracture mechanics techniques used, and superposition of creep and environmental effects could complicate mechanisms even more. The efforts of Manson (1966) attempt to bring together creep and fatigue by estimation of the fatigue characteristics at high temperatures, within the creep limit of materials. By assuming that the pre-existence of intercrystalline cracking has the effect of by-passing much of the crack initiation process, the number of cycles to failure is related to the crack propagation period. Claudio et al. (2004) present a numerical life prediction and failure analysis by means of a finite element technique and compare it with experimental results. Using a crack propagation program, with appropriate fatigue-creep crack growth rate data, fatigue life predictions were made.

Isothermal fatigue data continue to be used as a measure of ranking candidate alloys, but actual life prediction must involve further testing and modeling of the component. With adequate data and detailed analysis, the effects of LCF may be understood and crack growth rate can be predicted which allows for specified inspection intervals.

With regards to $\mathrm{HCF}$, the first task is to determine the natural frequencies of the component. Due to the complex shapes of the blades, a finite element analysis is typically performed to determine the modal shapes and frequencies. The designer must also determine the forcing frequencies caused by stator wakes or strut interaction. When a rotor blade passes across the wakes of the stator, it experiences fluctuating lift and moment forces repeatedly at a frequency dependant on the number of nozzles and the speed of the machine. These results are typically plotted on an interference or Campbell diagram, shown in Figure 2.14. 


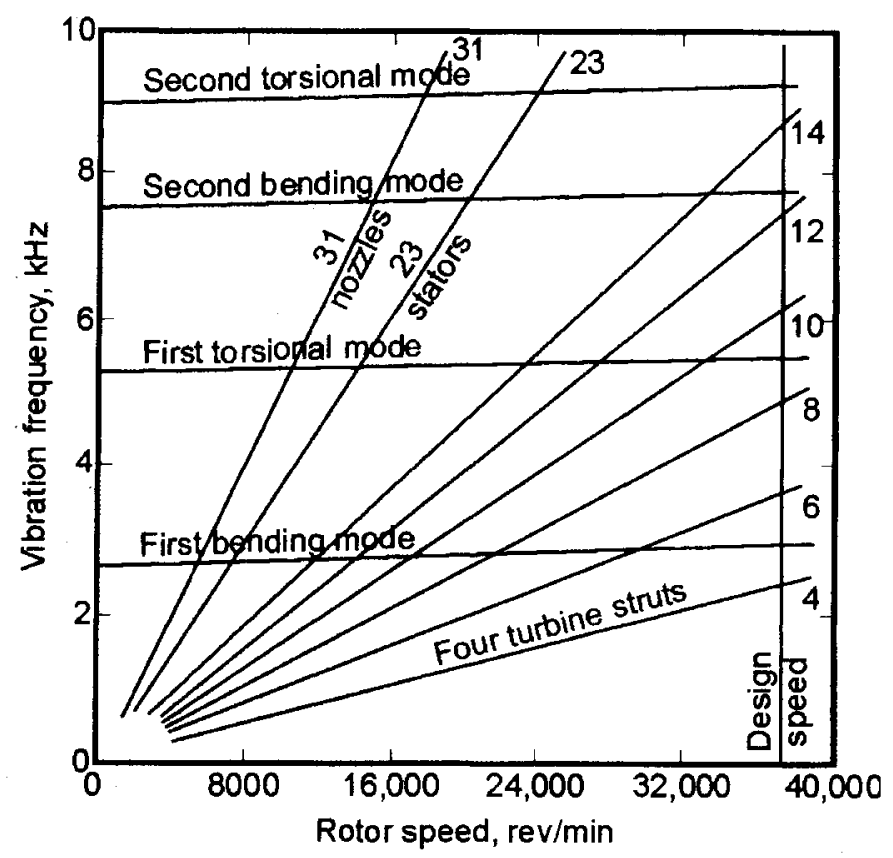

Figure 2.14: A typical interference diagram (Moustapha et al, 2003)

Here, the fundamental modes are plotted as nearly horizontal lines; a small upward shift tends to exist due to the stiffening from centrifugal effects. Diagonal lines represent the forcing functions caused by the presence of the nozzles or struts. Any intersections indicate the modes of vibration that will be excited by specific sources at particular rotational speeds. It is a standard, and a very advisable practice to avoid any of the first four orders of resonance. Additionally, the designer must recognize the effect of a superimposed mean stress on the effects of an alternating stress. Conventionally, this is displayed on a traditional Goodman diagram. A more recent version of this approach was put forward by Figge (1967). Rao (1991) presents an in-depth look at various analytical approaches for calculating blade vibration and life estimates.

Due to the complexity and importance of HCF estimation, Nichol (2003) presents an assessment of current HCF test approaches and identifies weaknesses. Kim et al. (2000) present a HCF analysis of a nickel base superalloy vane component. A comparison of the results of the fatigue test on the vane component with the S-N (stress to number of cycles to failure) curve of the bulk material is presented. 


\subsubsection{Corrosion}

Although LCF is the primary mechanism contributing to failure, in some instances, corrosion life can be reached long before the corresponding creep and fatigue limits. Corrosion failure occurs when the aerodynamic shape or tip clearances degrade to a limit in which replacement is warranted for performance or safety considerations. Careful control of fuel flow, inlet filtration, and use of inhibitors can reduce the effects of corrosion. Additionally, coatings are now being applied to turbine blades to protect hightemperature superalloys against corrosion and oxidation and allow designers to raise turbine inlet temperatures by 50 to 100 Kelvin, Gauthier (2005). Useful modeling for corrosion prediction require several independent variables such as metal and gas path temperature, coating chemistry and thickness, metal chemistry, and contaminant level in the combustion products. A complete overview of hot corrosion and protective coatings can be found in two separate chapters of Sims (1987). 


\subsection{Material Selection}

Undoubtedly, the current choice of material for the turbine components will be superalloys. For numerous years, the development of superalloys has allowed the turbine designer to take efficiency and power output to new levels. It has been the ever increasing demand for high-temperature materials that has lead to the evolution of a newer generation of superalloy materials. A variety of superalloys have been developed for elevated temperature service, demonstrating the combined properties of mechanical strength and surface stability required for high temperature engine components. Mangonon (1999), provides a general background for engineering material selection, while Rao (1996), offers a comparative evaluation of creep and stress rupture properties of some titanium alloys and superalloys.

Superalloys typically consist of 3 classes: nickel base, cobalt base, and iron base. While superalloys are typically designed for high strength, creep, and fatigue resistance at high temperatures, approximately 10-15 percent of these alloys have been developed for use in corrosion resistant applications, Sims et al (1987). The use of the gas turbine engine on helicopters in the late sixties resulted in the development of superalloys with improved oxidation and corrosion resistance as sea water ingestion caused corrosion problems in low chromium high pressure turbine blades.

\subsubsection{Superalloy Composition}

Chemically, the composition of nickel or cobalt base superalloys typically contains about 50 percent nickel and/or cobalt as the base alloy, 10-15 percent chromium for surface stability, and 4-8 percent of aluminum and titanium for precipitate formation and strengthening. The nickel base alloy matrix always consists of the close-packed FCC structure where solid solution strengthening and precipitation hardening contribute significantly to its mechanical capability. It is this close packed structure of superalloys that allows them to maintain relatively high and reliable tensile, rupture, creep, and thermomechanical fatigue properties, at high temperature application. The advent of 
directional solidification, and single crystal technology, has become very important to the gas turbine industry as it provides even further improved creep resistance to the alloy as well as enhanced mechanical properties. An additional benefit of superalloys is the low coefficient of thermal expansion, which allows tight tolerances as well as design clearances to be maintained thus maximizing component efficiency.

\subsubsection{Component Requirements}

A critical component for material selection is the 1st stage nozzle as it is subjected to the highest temperature. Temperature differentials cause high thermal stresses that cause low cycle thermal fatigue cracking. Additional properties required by first stage nozzles include low coefficients of thermal expansion, as well as high ductility and thermal conductivity.

Turbine rotor blades on the other hand, require significant creep capability. These blades are subject to extremely high stresses due to centrifugal loading and operate at extreme temperatures. The blades are also subject to high rates of alternating stresses due to the presence of upstream and downstream nozzles and struts. This leads to material requirements which include exceptional fatigue and creep strength. Additional requirements include high tensile strength and toughness. In extremely high temperature applications, in which cooling may be employed, the material will also require a highquality protective coating system and high temperature corrosion resistance.

In the case of turbine disks, the environment is usually governed by the sealing and cooling air and is usually not hotter than the compressor discharge temperature. According to Sims et al (1987), alloy steels are commonly utilized in industrial turbines, while Inconel 718 and similar alloys are found in aircraft engines. According to Radhakrishna and Rao (1994), Inconel 718 is one of the most widely used superalloys in the gas turbine jet engine industry, and accounts for approximately 35 percent of all wrought superalloy production. However, dimensional instability studies in machining this material require significant understanding of the metallurgical characteristics to 
control the deformation due to machining, as outlined by Subhas et al (2000). In turbine disks, the primary concern is disk burst which can occur when stress levels reach approximately 90 percent of the ultimate tensile stress. The main requirements for disk material and fabrication are therefore: good fracture toughness, low crack growth rate, and high probability for defect detection.

For applications requiring a low coefficient of thermal expansion (CTE), Heck et al. (1998) propose the use of Inconel 783. This material is an oxidation-resistant low CTE superalloy developed for gas turbine applications. Low-CTE tip and casing components maintain tight clearances throughout the operating range of the engine retaining turbine efficiency.

In searching for an applicable material, one must characterize the differences of failure mode and non-failure mode related data. Failure mode related data include corrosion data, creep and creep rupture, LCF and HCF curves, fracture mechanics data as well as yield and ultimate strength. On the other hand, non-failure mode related data is also required, as it is needed for stress, strain, and temperature calculations. This type of data includes Young's modulus, shear modulus, Poisson's ratio, coefficient of linear expansion, conductivity and density. 


\subsection{Computational Analyses}

As part of the preliminary design, a detailed structural and aerodynamic analysis is required. Currently, several commercial software packages are available for engineering analysis. As these software packages become more evolved, it becomes even more essential for the designer to understand the underlying physics and assumptions of these complex modeling tools. For this reason, a short discussion of computational fluid dynamics (CFD) and finite element analysis (FEA) is presented. An example of the Mach number distribution, calculated using CFX-5, within an axial stator-rotor stage is shown in Figure 2.15a. A finite element mesh used for computing the stress field for structural analysis is also shown, Figure $2.15 \mathrm{~b}$.

(a)

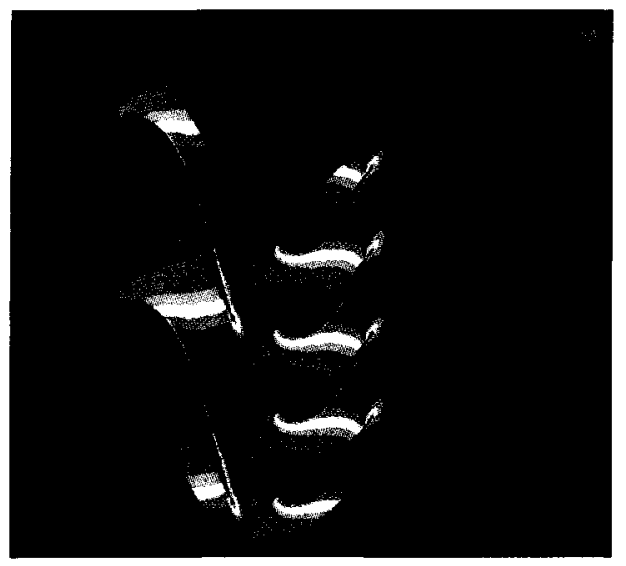

(b)

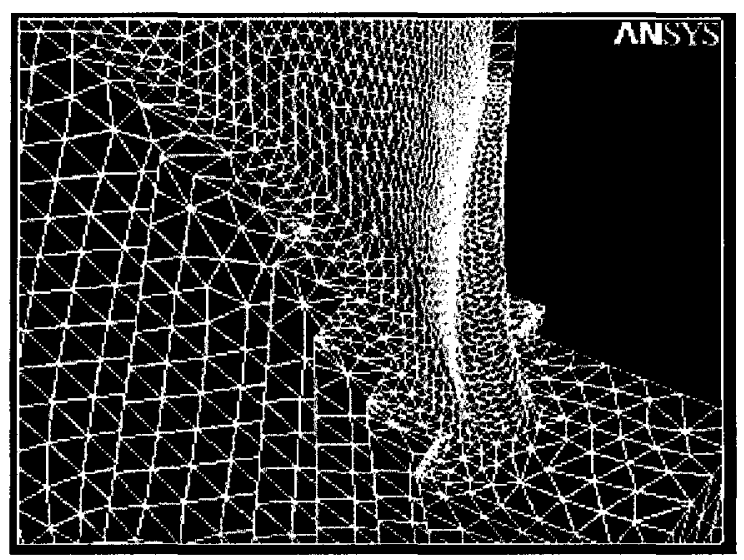

Figure 2.15: (a) Mach number distribution by CFD and (b) finite element mesh

\subsubsection{Computational Fluid Dynamics}

Computational fluid dynamics is based on the numerical solving of the equations of fluid flow over a region of interest, with specified conditions bounding that region. It allows the designer to estimate the aerodynamic performance of a given component, or it can give the designer better insight into the problems (separation, incidence, pressure loss) with an existing design. 
Most computational methods make use of the finite volume technique for solving the Navier-Stokes equations, which govern the fluid momentum, heat, and mass transport. In this technique, the region of interest is first divided into small sub-regions, called volumes. The equations are then discretised and solved iteratively over these small volumes to get an overall picture of the flow. Numerous researchers have applied these methods in a variety of ways for both analysis and verification of design. Demeulenaere et al. (1997) present a two-dimensional viscous inverse method for the design of compressor and turbine blades. The method solves the time-dependant Navier-Stokes equations in a numerical domain of which some boundaries (the blade walls) move during the transient part of the computation.

Due to the length scales of the finite volume method, additional considerations must be made for turbulence modeling. In this method, it is necessary to specify a turbulence model which seeks to solve a modified version of the Navier-Stokes equations, known as the Reynolds-averaged Navier-Stokes equations (RANS). This is done by introducing averaged and fluctuating turbulence quantities into the original transport equations.

Typically, turbulence models can be broken into two categories: eddy viscosity models and Reynolds stress models. Eddy viscosity models, such as the k-epsilon and SST model, are based on the idea of eddy formation and dissipation. A very basic form of eddy viscosity model, available in CFX-5, is known as a 'zero-equation' model. This model is very robust and computationally inexpensive as it uses a constant turbulent eddy viscosity for the entire domain. Dawes (1990) presents a comparison of zero- and oneequation turbulence modeling for turbomachinery calculations. The paper compares the performance of two standard models with experimental data for axial turbine secondary flow development. It was found that the more sophisticated model produces detail improvements in the local quality of the predictions, but as far as overall loss generation is concerned, it is concluded that there is little difference between the two models. 
Two-equation models are more widely used and generally provide more accurate results. These include models such as the k-epsilon model, a stable, numerically robust model (not recommended for separated flows), and the k-omega model. The k-omega based models of Menter (1994) were designed to give highly accurate predictions of typical engineering flows. The k-omega based SST model is recommended for high accuracy boundary layer simulations; however, when using it, it is advised to ensure that there are at least 10 points within the boundary layer to accurately resolve the velocity gradient. According to Menter (1994), the SST model leads to a significant improvement for all flows involving adverse pressure gradients and should be the model of choice for aerodynamic applications.

Reynold stress models, on the other hand, are more applicable to flows with strong swirl components or secondary flow. These models naturally include effects of streamline curvature, secondary flow or buoyancy. Reynold stress models offer better accuracy for these types of flow features as compared to the two turbulence models using the eddy-viscosity approximation. In addition to the commercial codes available, numerous variations of CFD codes are presented in the literature. Researchers have attempted to validate their own versions of these codes on various experimental results. For example, the work of Luo and Lakshiminarayana (1995) has resulted in an explicit two-dimensional Navier-Stokes code, with Chien's low Reynolds number k-epsilon model, Coakley's low Reynolds number q-omega model, and a two-layer kepsilon/ARSM (algebraic Reynolds stress model). The code was used to analyze the aerodynamics and heat transfer of a highly loaded transonic turbine cascade under various operation conditions. Reasonable predictions were obtained for heat transfer rates and the prediction of the onset of transition on the suction surface. Transition on the highly accelerating pressure side was not well predicted.

Near-wall flow modeling is extremely important in determining the location and severity of flow separation as it may have a strong implication on turbomachinery performance. The turbulence models listed previously can not accurately predict flow separation if the boundary layer is not accurately resolved. This has led to the 
development of scalable wall functions in CFX-5. The wall function method uses empirical formulas, such as the log law of the wall, that impose suitable conditions near the wall, without resolving the actual boundary layer. Traditionally, the designer had to specify whether to use standard wall functions or a low Reynolds number method. The low Reynolds number method required the boundary layer to be completely resolved by inflation layers. This method is suitable for turbulence models based on the omegaequation, such as the SST model. CFX-5 applies scalable wall function to all epsilon based models and automatic near wall treatment for k-omega based models. The automatic near-wall treatment will automatically switch from wall functions to a low-Re near-wall formulation as the mesh is refined. The application of CFD to turbine disk cavities is presented by Virr et al (1994). The code employs boundary-fitted coordinates and uses the k-epsilon turbulence model with alternative near-wall treatments. The work also compares the wall function approach and a one-equation near-wall model. Results indicate that conventional wall functions were found to give acceptable results for velocity and heat transfer predictions, provided the Reynolds number was high enough. It was noted that the one-equation model has a greater generality than wall functions; however, it required the use of more mesh points. The number of mesh points was still less than the required points for the low Reynolds number models described previously.

\subsubsection{Finite Element Analysis}

The finite element method is another numerical technique for the solution of boundary-value problems. The method was originally developed nearly 50 years ago in order to conduct structural analyses of complex objects which were too difficult to complete analytically. Like the finite volume method described previously, the object to be analyzed is represented by a geometrically similar model consisting of a finite number of sub-divided regions. Conventionally, many industrial organizations have made use of their own in-house codes. Today however, more and more companies are making use of the powerful tools available by commercial software developers. To date, numerous software packages, such as NASTRAN, ABAQAS, and ANSYS, are available. 
Much of the work that will follow includes the use of ANSYS for structural evaluation. ANSYS enables the engineer to build computer models or transfer CAD models of structures or components into the ANSYS environment for evaluation. Actual operating loads or design operating conditions may be simulated to study physical responses, such as stress levels, temperature distributions, or vibrational modes. Mathematically, the structure to be analyzed is subdivided into a mesh of finite sized elements. Within each element, the variation of displacement is assumed to be determined by simple polynomial shape functions and nodal displacements. Equations for the strains and stresses are developed in terms of the unknown nodal displacements. From this, the equations of equilibrium are assembled in a matrix form which can easily be programmed and solved using a computer. After applying the appropriate boundary conditions, the nodal displacements are found by solving the matrix stiffness equation. Once the nodal displacements are known, element stresses and strains can be calculated.

Subramani et al (1995) have carried out a finite element analysis on a typical automotive turbocharger turbine wheel using the concept of cyclic symmetry. The paper presents both the solution and the approach that were used in determining the turbine disk stresses and blade vibration. Modern software packages are beginning to allow designers to conduct high quality multi-disciplinarily coupled simulations. Watanabe et al. (2004) have presented a manual coupling of the CFD software CFX-TASCflow and FEA software NASTRAN. Blade geometry, static pressure, and static temperature distribution, required for finite element analysis were obtained by CFD calculation. Similarly, Filsinger et al (2002) describe an approach to unidirectional coupled CFD-FEA simulations. These authors were concerned with HCF of axial turbocharger turbine blades. To predict the excitation forces acting on the rotating blades, the time-resolved two-dimensional coupled stator-rotor field of the stage was calculated. The unsteady pressure leads to pulsating excitation forces acting on the rotor which are transferred to the finite element model. As a result of the unidirectional coupled model, the authors were able to obtain a structural optimization from the resulting fluid flow. 


\subsection{Mechanical Design}

At this point in the design process, the designer should have a basic comprehension the overall layout of the turbine components. The blade profile and geometry have been set, and it is now the responsibility of the designer to effectively transfer the energy extracted from the flow into shaft power. This will require the design of the turbine disk and blade attachment mechanism. Both of these processes are outlined in detail by Sawyer (1985). For aircraft applications, the weight of the turbine rotor is of great importance. To minimize weight, while at the same time maintaining stringent safety considerations, this component must be carefully designed. A disk burst is potentially the most catastrophic failure possible in an engine and therefore disks are designed with overspeed capability and low cycle fatigue as primary objectives. Analyses of centrifugal stresses in turbine disks are presented by Haigh and Murdoch (1963), and Fessler and Thorpe (1968). Tong et al (2004) present an automated engine design tool, which easily allows engine developers to perform design tradeoffs between engine performance, durability, and cost. Within the program, the stress distribution for various disk geometries is also incorporated in order for a life-prediction module to calculate disk life.

Most commonly, gas turbine blades are attached to the turbine disk by a fir-tree or dovetail configuration. A modern turbine blade with fir-tree attachment is shown in Figure 2.16.

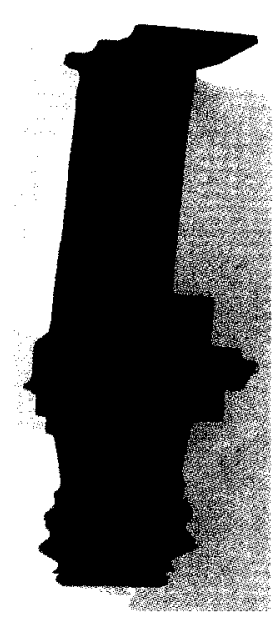

Figure 2.16: Allison T56 first stage turbine blade with fir-tree root 
Details of the methods for blade attachment exist in the literature. Shiratori et al (1967) derive a method of elastic design of fir-tree joints for blade fastenings under centrifugal tensile loading. The method is mathematically complex and is listed only for completeness. Shaw (1970) presents an improved blade root design for axial flow compressors and turbines. Flexible blade roots and blade twisting effects are investigated. Meguid et al (2000) have conducted comprehensive two-dimensional and three-dimensional finite element analyses of the fir-tree region in turbine disks. The study acts as a valuable design aid as it fully examines the effect of the critical geometrical features, such as the number of teeth, flank length and flank angle upon the stress field in the disk.

Unfortunately, this approach of mechanically attaching blades via fir-tree or dovetail configured attachments, limits rotor speed because of the stress concentrations in the disk rim. Although there are already bladed disks in service, Cairo and Sargent (2002), present the idea of creating an integrally bladed rotor in which blades are integral with the disk. This can be achieved by metallurgically bonding the blades to the disk rim or simply machining it as one piece. The paper discusses how a scientific approach and creative design practice can lead to such a process.

An overview of axial turbine design methods that exist in literature has been presented. The literature review has also described the theories and principles that govern the flow and structural considerations of the axial flow turbine. Using the reviewed material and most widely accepted methods, a preliminary design tool for the complete aerodynamic and structural design of axial flow turbines will be presented next. The tool was created to provide an up-to-date generic design tool for use in the development of axial flow turbine geometry. It enables the designer to obtain a fast estimate of performance and a fast screening of various design variables. The following chapter will describe, in detail, the methods used in the tool. Subsequent chapters will provide verification of the design tool using CFD and FEA. Future tasks will include the verification of these methods by experimental testing, which was not the focus of this thesis. 


\section{Chapter 3}

\section{Aerodynamic and Structural Design Tool}

A comprehensive design tool was created to facilitate in the development of two axial flow turbines for a 1-MW gas turbine engine. The tool consists of a series of steps, methods, and equations programmed into a MATHCAD worksheet to automate the aerodynamic and structural design process of a given gas turbine stage. A single stage gas generator turbine and a single stage power turbine were designed using this tool and will be described in subsequent chapters. This section will present the underlying methods and equations used within the design tool. A general overview of the design algorithm and its functionality is presented first. Following this, a more detailed look at each of the sections within the program will be presented. It should be noted that the design tool is only capable of producing the preliminary design geometry based on a competent selection of the initial parameters. A brief review and background of these parameters will be given herein. 


\subsection{Overview}

In a general sense, the program requires a selected number of initial design parameters and operating conditions (which will be described further) and produces the preliminary geometry for a given gas turbine stage. The operating conditions required for the design are entered by the user. These include such conditions as: turbine inlet and outlet temperature and pressure, mass flow rate, and the work output required by the stage. The fluid type and material properties are also entered. From this point, the user is required to enter a selected number of design parameters which will govern the design. A summary of the design algorithm is shown in Figure 3.1. 


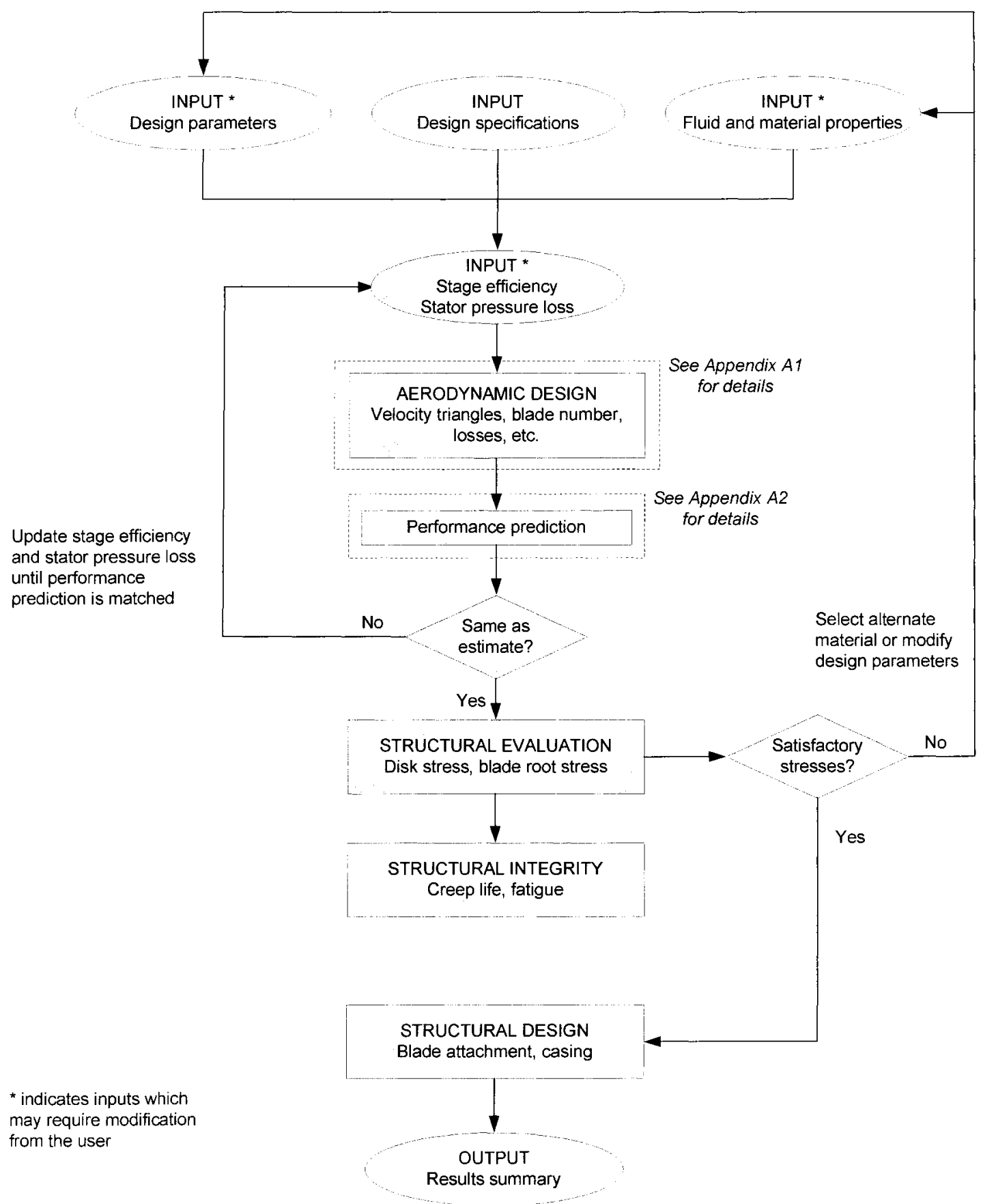

Figure 3.1: Design tool flow chart 
First, the program conducts a series meanline velocity calculations required for the given operating conditions and work output. Using a free-vortex design approach, the program then calculates the velocity diagrams for the hub and tip to ensure sufficient reaction and loading at each location. For a given aspect ratio and Zweifel loading coefficient, the number of stator and rotor blades are then calculated. Using the method outlined by Kacker and Okapuu (1982), an aerodynamic performance prediction is conducted. For structural considerations, the program conducts a blade and disk stress estimation. Using the blade stress and surface temperature, an evaluation of the creep life is then conducted by the method of Larsen and Miller (1952). Using estimates for the gas bending stresses, the possibility of fatigue failure is investigated. Assuming the stress estimates are satisfactory, a preliminary fir-tree blade attachment sizing is completed. Finally, a burst speed calculation and casing thickness estimate are performed.

The program requires manual iteration to obtain the overall efficiency and stator pressure loss. The iteration is a result of the performance prediction method used in the design. The program requires the user to provide an initial guess for the stage performance and stator pressure loss. Based on the input data, the program then calculates the actual performance and pressure loss. These values are then re-entered in place of the initial guess. Typically only one or two iterations are required which may be easily automated if desired. Appendices A1 and A2 provide additional detailed flow charts for the highlighted regions in Figure 3.1. Manual iteration may also be required as a result of the blade and disk stress estimation. A poor material selection or inadequate design parameters may yield an undesirable creep life or significantly high stresses. In these cases, a new material must be selected (and the material properties updated) or the design parameters must be modified.

A number of assumptions were included in the development of the preliminary design tool. For aerodynamic considerations, constant fluid properties were assumed. These properties were obtained from the performance and design specifications. They are an average of the inlet and outlet values. It is also assumed that the flow is entering the first stage without circumferential or radial variation. The flow is assumed to enter 
the first stator row at 0 degrees from the axial direction. The temperature distribution is also assumed to be constant. Based on these assumptions, it is given that the stagnation enthalpy is also constant over the annulus, as required by the free-vortex design. For preliminary design purposes, the performance prediction method is assumed only to apply for the design conditions. Furthermore, the performance prediction method is assumed to be a combination of profile, secondary, trailing edge, and tip clearance losses. In the event of supersonic exit velocities, the drag rise factor of the profile loss calculation is assumed to include the effects of trailing edge losses. For structural considerations, a uniform thin disk is assumed for the calculation of the rotor disk stress distribution. The blade metal temperatures for the heat transfer analysis and the creep life estimation are assumed to be on the order of magnitude of the local static temperature. For the blade attachment design, the platform and fir-tree depth are assumed to be logical fractions of the blade height. Three fir-tree teeth are assumed based on various available blade design data. 


\subsection{Aerodynamic Design}

This section will provide the reader with details of each of the various steps required to reach the preliminary aerodynamic design. Selected portions of the code will be presented throughout to give an overall understanding of the capability of the program. The inputs will be described first followed by the aerodynamic and structural design algorithms.

\subsubsection{Design Parameters}

The program requires the user to select values for a number of specified parameters pertaining to the design. These parameters are commonly used in gas turbine designs and can be found in nearly any gas turbine design textbook. The selected parameters form a basis for the gas turbine preliminary design and also govern its overall performance, feasibility, and application. These parameters are explained briefly below and summarized in Table 3.1. Information regarding all input requirements, as well as many of their respective typical values may be found in Appendix B1.

\begin{tabular}{|c|c|}
\hline$\psi$ & Stage loading coefficient \\
\hline$\varphi$ & Flow coefficient \\
\hline$\Lambda$ & Degree of reaction \\
\hline $\mathrm{C}_{\mathrm{D}}$ & Discharge coefficient \\
\hline $\mathrm{C}_{\mathrm{L}}$ & Zwiefel lift coefficient \\
\hline $\mathrm{AR}$ & Aspect ratio \\
\hline$\alpha_{1}$ & Inlet swirl angle [deg] \\
\hline $\mathrm{N}$ & Rotational speed [RPM] \\
\hline
\end{tabular}

Table 3.1: Summary of design tool parameters

Most importantly, the designer must select a stage loading $(\psi)$ and flow coefficient $(\varphi)$ compatible with the design. Stage loading is a measure of the overall work output of a given turbine stage. The flow coefficient, on the other hand, is a measure of the velocity levels within the stage. According to Smith (1965), for a given 
stage loading coefficient there is an optimum level of flow coefficient. From these parameters alone, an estimate of the overall stage efficiency can be obtained by use of the Smith chart, shown in the previous chapter (see Figure 2.6). It should be noted that these efficiencies have been adjusted to exclude tip clearance loss; therefore, these efficiencies are higher than would be expected in similar turbines in operation. According to Moustapha et al. (2003), the Smith chart does not provide a good indication of the actual efficiency of a given design; however, the comparison between blade rows of different loading and flow coefficients is generally found to be quite reliable. With this in mind, suitable values of stage loading and flow coefficient can be selected and entered into the program.

Additionally, the user must specify a desired degree of reaction $(\Lambda)$. The degree of reaction defines how the complete turbine stage expansion is split between the rotor and stator. A high reaction indicates a large amount of expansion in the rotor; whereas, a low reaction implies a large expansion in the stator. The choice of reaction also has implications on the mechanical design of the blade. Additional discussion on the selection of blade reaction has been included in Section 2.2.1.

The user is also required to input a value for the discharge coefficient $\left(C_{D}\right)$. The discharge coefficient is used to relate the effective area to the geometrical area. This difference in area is strongly related to the effects of trailing edge and boundary layer blockage. This coefficient is not critical in turbine design, where the accelerating flow and favourable pressure gradients cause boundary layers to remain much thinner than in other types of turbomachinery. A typical range for turbine design is $0.95-0.98$, meaning that 2-5 percent of the geometrical area is blocked.

In order to determine the number of blades and their respective shape, values for the Zweifel lift coefficient and aspect ratio must also be entered. Typical values have been previously presented, and their use in the program will be explained later. Finally, the user must select a suitable rotational speed and inlet flow angle. Typically, the first turbine stage is designed for zero inlet swirl, $\left(\alpha_{1}=0\right)$. However, for multi-stage turbines 
there is typically some degree of outlet swirl from a previous stage which will need to be accounted for in the design of subsequent stages. The selection of rotational speed will have a direct impact on the size of the machine. With the work output set, and the stage loading selected, the mean radius of the machine will depend directly on the rotational speed.

At this point, the design flow conditions and design parameters have been set and entered into the program. An initial guess must be made for the isentropic efficiency and stator pressure loss. These will need to be updated manually after they have been calculated in a later section.

\subsubsection{Specific Speed}

In the event of the design of a new engine, the turbine configuration is not set. In this case, the designer will typically refer to the specific speed for the selection of the turbomachinery configuration. For this reason, a specific speed calculation is conducted at the onset of the program. For turbine design, the specific speed $\left(N_{s}\right)$ in its nondimensional form is given by:

$$
\mathrm{N}_{\mathrm{S}}=\omega \cdot \frac{\sqrt{\frac{\mathrm{m}_{\text {air }} \cdot \mathrm{R}_{\text {air }} \cdot \mathrm{T}_{03 \mathrm{~d}}}{\mathrm{P}_{03 \mathrm{~d}}}}}{\left|\Delta \mathrm{h}_{0}\right|^{3 / 4}}
$$

where $\omega$ is the rotational speed in $\mathrm{rad} / \mathrm{s}, \mathrm{R}_{\text {air }}$ is the gas constant for air, and $\mathrm{m}_{\text {air }}$ is the mass flow rate of air (or gas). $\mathrm{T}_{03 \mathrm{~d}}$ and $\mathrm{P}_{03 \mathrm{~d}}$ represent the design point total temperature and pressure at the stage outlet. Finally $\Delta \mathrm{h}_{0}$ is defined as the change in enthalpy required for the specified work output. The variation of turbine stage efficiency with specific speed has been shown previously, Figures 2.1 and 2.3. 


\subsubsection{Velocity Triangles}

Construction of the velocity triangles begins by determining the amount of swirl required to produce the desired work output. Using the stage loading $(\psi)$ and flow coefficients $(\varphi)$, in conjunction with the required enthalpy change, the mean blade speed $\left(\mathrm{U}_{\mathrm{m}}\right)$ and axial velocity component $\left(\mathrm{C}_{\mathrm{x}}\right)$ can be calculated as follows:

$$
\begin{aligned}
& \mathrm{U}_{\mathrm{m}}=\sqrt{\frac{\left|\Delta \mathrm{h}_{0}\right|}{\psi}} \\
& \mathrm{C}_{\mathrm{x}}=\varphi \cdot \mathrm{U}_{\mathrm{m}}
\end{aligned}
$$

Using the calculated blade speed, the change in swirl velocity $\left(\Delta \mathrm{C}_{\theta}\right)$ required can be calculated as follows:

$$
\Delta \mathrm{C}_{\theta}=\frac{\left|\Delta \mathrm{h}_{0}\right|}{\mathrm{U}_{\mathrm{m}}}
$$

The velocity triangles may now be constructed by determining the flow velocity at each station. The stations and their sign conventions are given in Figure 3.2. 


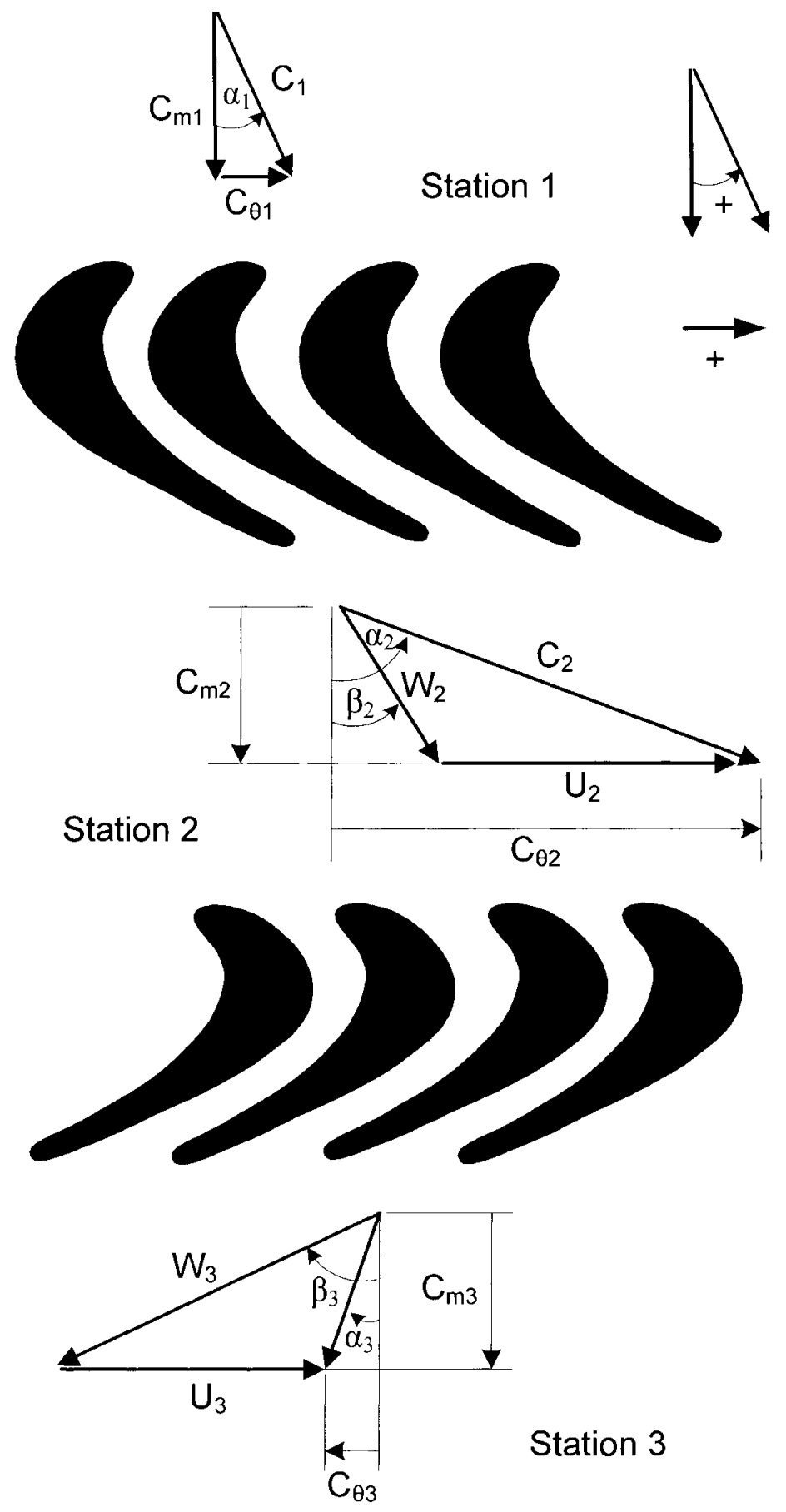

Figure 3.2: Velocity diagram and station numbering 
The methods and equations used in this section are largely based on those presented by Japikse and Baines (1997). Once the values of $\psi, \varphi$, and $\Lambda$ have been selected, the blade angles follow immediately. It can be shown by Japikse and Baines (1997) that the relative outlet flow angle $\left(\beta_{3}\right)$ of the rotor can be given by:

$$
\beta_{3}=\arctan \frac{-(\psi+2 \Lambda)}{2 \varphi}
$$

The program then determines the velocity components at the rotor outlet (Station 3). For constant axial velocity:

$$
\mathrm{C}_{\mathrm{x} 3}=\mathrm{C}_{\mathrm{x}}
$$

The remaining components are simply calculated by basic trigonometry. It is essential to ensure the correct sign convention for positive and negative as shown in Figure 3.2. The outlet swirl component $\left(\mathrm{C}_{\theta 3}\right)$ and the swirl angle $\left(\alpha_{3}\right)$ in the absolute reference frame are given by:

$$
\begin{aligned}
& \mathrm{C}_{\theta 3}=\mathrm{U}_{\mathrm{m}}-\mathrm{W}_{3} \cdot \sin \left(\beta_{3}\right) \\
& \alpha_{3}=\arctan \frac{\mathrm{C}_{\theta 3}}{\mathrm{C}_{\mathrm{x} 3}}
\end{aligned}
$$

The corresponding absolute $\left(\mathrm{C}_{3}\right)$ and relative $\left(\mathrm{W}_{3}\right)$ velocities are then:

$$
\begin{aligned}
& \mathrm{C}_{3}=\sqrt{\mathrm{C}_{\mathrm{x} 3}{ }^{2}+\mathrm{C}_{\theta 3}^{2}} \\
& \mathrm{~W}_{3}=\frac{\mathrm{C}_{\mathrm{x} 3}}{\cos \left(\beta_{3}\right)}
\end{aligned}
$$


Next, the rotor inlet components may be calculated. Once again, for constant axial velocity:

$$
\mathrm{C}_{\mathrm{x} 2}=\mathrm{C}_{\mathrm{x}}
$$

Additionally, the required inlet swirl component and flow angle, measured in the absolute reference frame, are given by:

$$
\begin{aligned}
& \mathrm{C}_{\theta 2}=\Delta \mathrm{C}_{\theta}+\mathrm{C}_{\theta 3} \\
& \alpha_{2}=\arctan \frac{\mathrm{C}_{\theta 2}}{\mathrm{C}_{\mathrm{x} 2}}
\end{aligned}
$$

The rotor inlet relative flow angle is then given by:

$$
\beta_{2}=\arctan \frac{-\left(\mathrm{U}_{\mathrm{m}}-\mathrm{C}_{\theta 2}\right)}{\mathrm{C}_{\mathrm{x} 2}}
$$

The absolute and relative velocities at the rotor inlet are then given by:

$$
\begin{aligned}
& \mathrm{C}_{2}=\sqrt{\mathrm{C}_{\mathrm{x} 2}{ }^{2}+\mathrm{C}_{\theta 2}{ }^{2}} \\
& \mathrm{~W}_{2}=\frac{\mathrm{C}_{\mathrm{x} 2}}{\cos \left(\beta_{2}\right)}
\end{aligned}
$$

The stator inlet velocity components are finally given by:

$$
\mathrm{C}_{\mathrm{x} 1}=\mathrm{C}_{\mathrm{x}}
$$




$$
C_{1}=\frac{C_{x 1}}{\cos \left(\alpha_{1}\right)}
$$

where $\alpha_{1}$ is specified by the designer.

\subsubsection{Fluid State Calculations}

In order to calculate the various temperatures $(\mathrm{T})$ and pressures $(\mathrm{P})$, it is necessary to make use of the isentropic flow relations for perfect gases. Equations 3.19-22 will summarize the functions used to convert these total quantities to their corresponding static values and vice-versa.

$$
\mathrm{T}_{0}=\mathrm{T} \cdot\left[1+\frac{\gamma-1}{2} \cdot \mathrm{M}^{2}\right]
$$

or

$$
\begin{aligned}
& \mathrm{T}=\frac{\mathrm{T}_{0}}{1+\frac{\gamma-1}{2} \cdot \mathrm{M}^{2}} \\
& \mathrm{P}_{0}=\mathrm{P} \cdot\left[1+\frac{\gamma-1}{2} \cdot \mathrm{M}^{2}\right]^{\frac{\gamma}{\gamma-1}}
\end{aligned}
$$

or

$$
\mathrm{P}=\frac{\mathrm{P}_{0}}{\left[1+\frac{\gamma-1}{2} \cdot \mathrm{M}^{2}\right]^{\frac{\gamma}{\gamma-1}}}
$$

In the above relations, $\mathrm{M}$ is defined as the Mach number and $\gamma$ is defined as the ratio of specific heats. For the first iteration, the estimation of the stator pressure loss $\left(\Delta \mathrm{P}_{0}\right)$ is entered by the user. This is required for the program to calculate the total pressure at the entry to the rotor. Since no work is done by the stators, the total temperature change 
across them is negligible. For a given percentage pressure loss $(\% \mathrm{PL})$, the calculation is as follows:

$$
\begin{aligned}
& \Delta \mathrm{P}_{0}=\% \mathrm{PL} \cdot \mathrm{P}_{01} \\
& \mathrm{P}_{02}=\mathrm{P}_{01}-\Delta \mathrm{P}_{0} \\
& \mathrm{~T}_{02}=\mathrm{T}_{01}
\end{aligned}
$$

Based on the total enthalpy required by the design specifications and the estimated isentropic efficiency $(\eta)$ entered by the designer, an estimate of the total temperature and pressure at the rotor exit can be calculated as follows:

$$
\begin{aligned}
& \mathrm{T}_{03}=\mathrm{T}_{02}+\frac{\Delta \mathrm{h}_{0}}{\mathrm{C}_{\mathrm{p}}} \\
& \mathrm{P}_{03}=\mathrm{P}_{01} \cdot\left[1-\frac{1}{\eta} \cdot\left(1-\frac{\mathrm{T}_{03}}{\mathrm{~T}_{01}}\right)\right]^{\frac{\gamma}{\gamma-1}}
\end{aligned}
$$

where $C_{p}$ is defined as the specific heat. Using the absolute velocity components and the total temperature at each station, the program then calculates the absolute Mach numbers at each station as follows:

$$
M=\sqrt{\frac{C^{2}}{\gamma \cdot R_{\text {air }} \cdot T_{0}-C^{2} \cdot \frac{\gamma-1}{2}}}
$$

Using the Mach number and total temperature, the static temperature at each station may also be calculated. This is done using the isentropic relation shown in Equation 3.20. 
The magnitude of the relative Mach numbers $\left(\mathrm{M}_{\mathrm{rel}}\right)$ can then be calculated using the static temperature and relative velocity, as shown in Equation 3.29.

$$
\mathrm{M}_{\mathrm{rel}}=\frac{\mathrm{W}}{\sqrt{\gamma \cdot \mathrm{R}_{\mathrm{air}} \cdot \mathrm{T}}}
$$

\subsubsection{Blade Sizing}

The next step in the design is the sizing of the blades. This begins by determining the meanline radius required by the design specifications. The mean blade radius $\left(\mathrm{r}_{\mathrm{m}}\right)$ can be determined by Equation 3.30. The area (A) required to pass the specified mass flow is

given by Equation 3.31. Here, the calculation takes into account the axial component of velocity, static density $(\rho)$, as well as the discharge coefficient $\left(\mathrm{C}_{\mathrm{D}}\right)$, described previously in Section 3.2.1.

$$
\begin{aligned}
& \mathrm{r}_{\mathrm{m}}=\frac{\mathrm{U}_{\mathrm{m}}}{\omega} \\
& \mathrm{A}=\frac{\mathrm{m}_{\mathrm{air}}}{\mathrm{C}_{\mathrm{D}} \cdot \rho \cdot \mathrm{C}_{\mathrm{x}}}
\end{aligned}
$$

where the density is given by the ideal gas law:

$$
\rho=\frac{P}{R_{\text {air }} \cdot T}
$$

Assuming that the annular area of the flow path and mean radius are given by Equations 3.33 and 3.34:

$$
\mathrm{A}=\pi \cdot\left(\mathrm{r}_{\mathrm{t}}^{2}-\mathrm{r}_{\mathrm{h}}^{2}\right)
$$




$$
r_{m}=r_{h}+\frac{r_{t}-r_{h}}{2}
$$

the hub and tip radii at each station is given by:

$$
\begin{aligned}
& r_{h}=r_{m}-\frac{A}{4 \cdot \pi \cdot r_{m}} \\
& r_{t}=2 \cdot r_{m}-r_{h}
\end{aligned}
$$

The hub-to-tip ratio is then calculated for mechanical design considerations.

\subsubsection{Stagger Angle and Maximum Thickness}

For preliminary design purposes, estimates of stagger angle $(\Phi)$ and maximum thickness-to-chord $\left(t_{\max } / c\right)$ ratio are obtained from Kacker and Okapuu (1982). For given inlet and outlet angles, they suggest 'optimal' values which have been based on previous designs. Curve fits for these values have been obtained from Sjolander (2002). The curve fits have been implemented into the program to automate the design process. Curve fit data for Figure 3.3(a) is given in Appendix C1. 
(a)

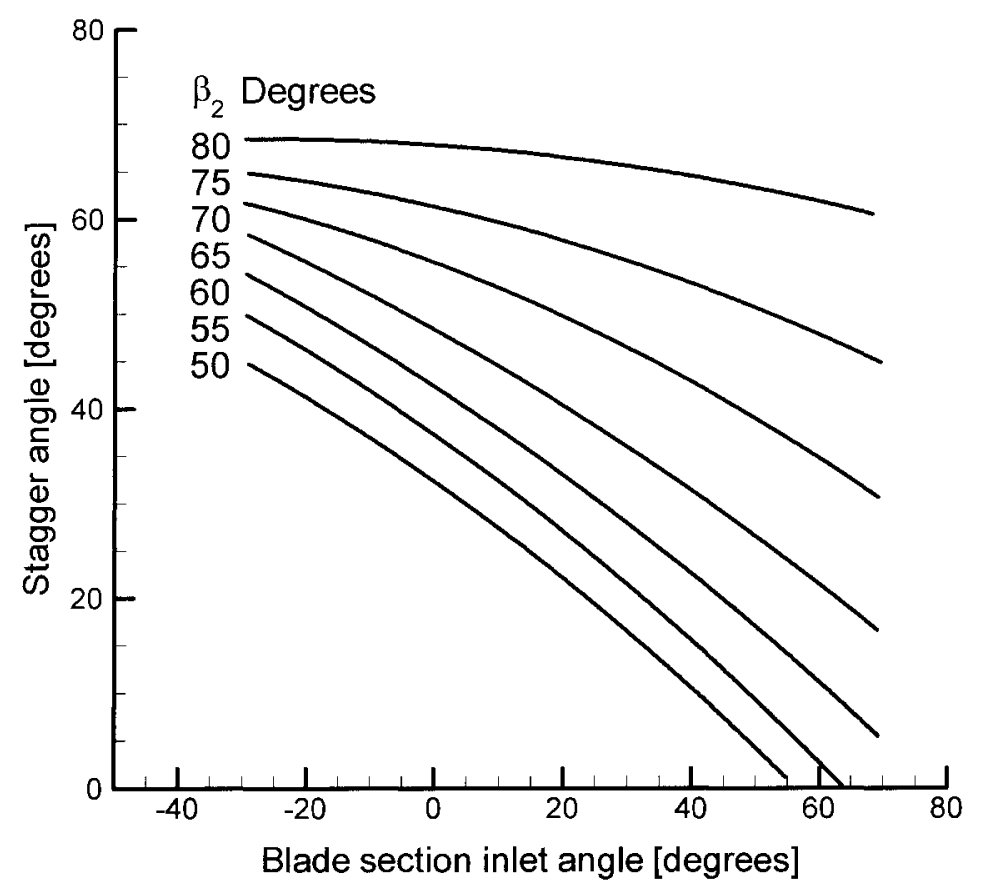

(b)

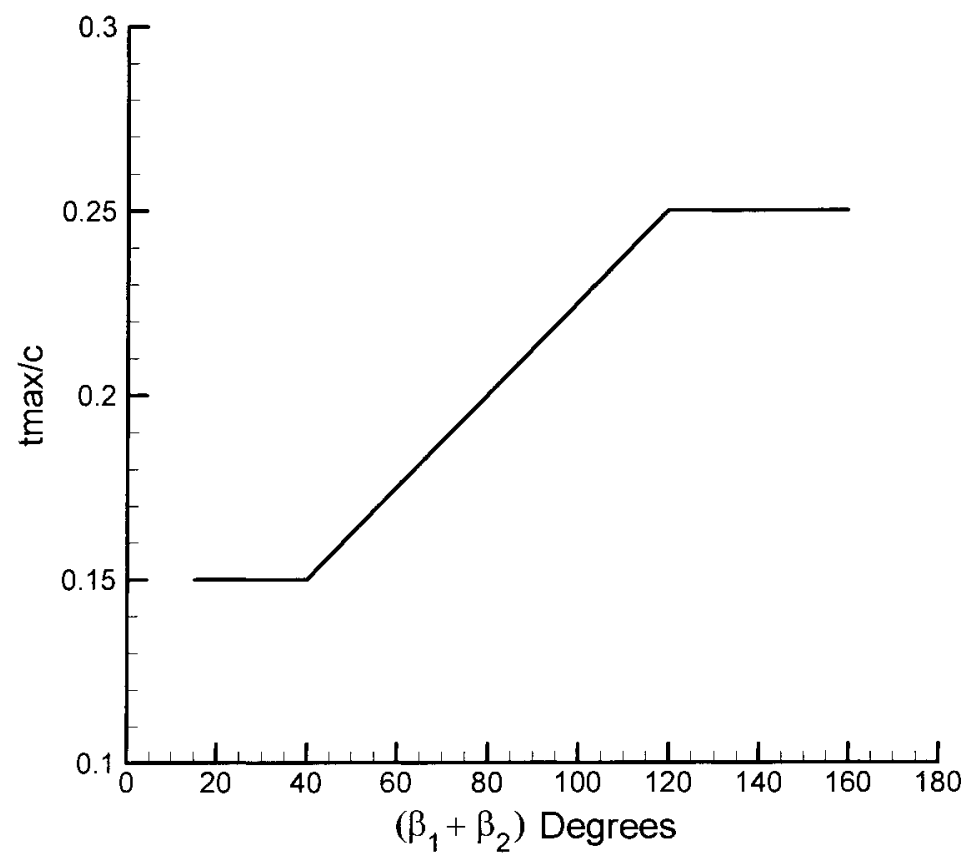

Figure 3.3: (a) Stagger angle for typical turbine blade sections and (b) Thickness-to-chord ratio for typical turbine blade sections (Adapted from Kacker and Okapuu, 1982) 


\subsubsection{Three-Dimensional Design}

To this point in the tool, all of the design has been conducted at the blade mean radius. From this point, a radial component will be added to the design by determining the velocity components and blade angles at the hub and tip radii. The design accounts for the radial variation using what is known as a free-vortex stage. As explained previously, a free-vortex stage has a constant axial velocity and a swirl component of velocity that is inversely proportional to the radius. The equations used for the calculation of a free-vortex stage are given by:

$$
\mathrm{C}_{\theta}=\text { constant } / \mathrm{r}
$$

where, constant $=\mathrm{C}_{\theta \mathrm{m}} / \mathrm{r}_{\mathrm{m}}$

$$
\mathrm{U}=\text { constant } \cdot \mathbf{r}
$$

where, constant $=\mathrm{U}_{\mathrm{m}} / \mathrm{r}_{\mathrm{m}}$

Using these equations, the blade speed and swirl component of velocity are calculated for the hub and tip radii. Due to the radial variation in velocity components, the stage loading, flow coefficient, and degree of reaction at the hub and tip will differ from the meanline design parameters. If these parameters drift too far from those specified at the meanline, the overall performance of the blade row could be severely affected. Using the new velocity components, the calculation of the rotor outlet relative blade angle will now be given by:

$$
\beta_{3}=\arctan \frac{-\left(\mathrm{U}_{3}-\mathrm{C}_{\theta 3}\right)}{\mathrm{C}_{\mathrm{x} 3}}
$$

where $\mathrm{U}_{3}$ and $\mathrm{C}_{\theta 3}$ are calculated for the hub and tip by the free-vortex equations, given in Equations 3.37 and 3.38. The additional calculations for the hub and tip velocity 
diagrams remain the same as the meanline calculations shown previously, substituting the appropriate values of $\mathrm{U}$ and $\mathrm{C}_{\theta}$ as required. Next, the reaction and stage loading at the hub and tip regions are calculated to ensure that the amount of stage loading is not excessive. For high pressure turbine designs, a stage loading less than 2.5 and a positive reaction are recommended. The reaction and stage loading are verified using the following equations:

$$
\begin{aligned}
& \Lambda_{\mathrm{h}}=\frac{\frac{1}{2} \cdot\left[\left(\mathrm{U}_{\mathrm{h} 3}{ }^{2}-\mathrm{U}_{\mathrm{h} 2}{ }^{2}\right)+\left(\mathrm{W}_{\mathrm{h} 2}{ }^{2}-\mathrm{W}_{\mathrm{h} 3}{ }^{2}\right)\right]}{\Delta \mathrm{h}_{0}} \\
& \psi_{\mathrm{h}}=\frac{\left|\Delta \mathrm{h}_{0}\right|}{\mathrm{U}_{\mathrm{h} 3}{ }^{2}}
\end{aligned}
$$

Finally, the absolute and relative Mach numbers are calculated for the hub and tip regions, as shown in Equations 3.28 and 3.29.

\subsubsection{Blade Number Estimation}

The next step in the design process is to estimate the number of stator and rotor blades required to efficiently expand or accelerate the fluid. The number of blades required for the design has a strong dependence on the selection of the Zweifel (or lift) coefficient determined previously. Additional considerations include the amount of blade turning, stagger angle, and aspect ratio. Using the Zweifel lift coefficient given by Equation 3.42, the solidity $(\sigma)$ of the blade row is calculated by Equation 3.43,

$$
\begin{aligned}
& \mathrm{C}_{\mathrm{L}}=2 \cdot\left(\frac{\mathrm{s}}{\mathrm{c}_{\mathrm{x}}}\right) \cdot \cos \left(\alpha_{\mathrm{ex}}\right)^{2} \cdot\left[\tan \left(\alpha_{\mathrm{in}}\right)+\tan \left(\alpha_{\mathrm{ex}}\right)\right] \\
& \sigma=\frac{2 \cdot \cos \left(\alpha_{\mathrm{ex}}\right)^{2} \cdot\left[\tan \left(\alpha_{\mathrm{in}}\right)+\tan \left(\alpha_{\mathrm{ex}}\right)\right]}{\mathrm{C}_{\mathrm{L}}}
\end{aligned}
$$


where the solidity is given by:

$$
\sigma=\frac{\mathrm{c}_{\mathrm{x}}}{\mathrm{s}}
$$

Using the specified aspect ratio (AR), defined as:

$$
\mathrm{AR}=\frac{\mathrm{h}}{\mathrm{c}}
$$

the true chord (c) of the blade can be calculated from the average blade height (h) determined earlier. Using the true chord and stagger angle, the axial chord may be estimated by:

$$
\mathrm{c}_{\mathrm{x}}=\mathrm{c} \cdot \cos (\Phi)
$$

The spacing (s) can then be calculated by Equation 3.44. Finally, the number of blades (n) required by the design can be determined from:

$$
\mathrm{n}=\frac{2 \cdot \pi \cdot \mathrm{r}_{\mathrm{m}}}{\mathrm{s}}
$$

For later use, the maximum blade thickness may now be calculated from the maximum thickness-to-chord ratio determined in Section 3.2.6.

\subsubsection{Incidence and Deviation}

In the design of gas turbine blading, attention must be paid to the difference in flow angles and blade metal angles. To this point, all of the calculations pertain to the flow angles required to achieve the design specifications. Consideration must be made for the incidence and deviation that occur at the blade leading and trailing edges, 
respectively. By definition, the difference between the blade angle $\left(\beta^{\prime}\right)$ and the flow angle $(\beta)$ at the leading edge is known as incidence (i):

$$
\mathrm{i}=\beta_{2}-\beta_{2}^{\prime}
$$

and the difference between the two at the trailing edge is known as deviation $(\delta)$ :

$$
\delta=\beta_{3}-\beta_{3}^{\prime}
$$

These quantities are labelled in Figure 3.4. For a stator blade however, $\beta_{2}$ and $\beta_{3}$ will become $\alpha_{1}$ and $\alpha_{2}$, respectively. It is important that the designer ensure that the sign of the value being used is compatible with the sign convention shown in Figure 3.2. For example, the deviation at the design point will always tend to 'under turn' the flow. Thus, depending on the sign of the outlet flow angle, the deviation may be assumed positive or negative. For the rotor blade section shown in Figure 3.2, the design incidence will be positive, and the design deviation will be negative. This is also illustrated in Figure 3.4. The throat opening is also defined.

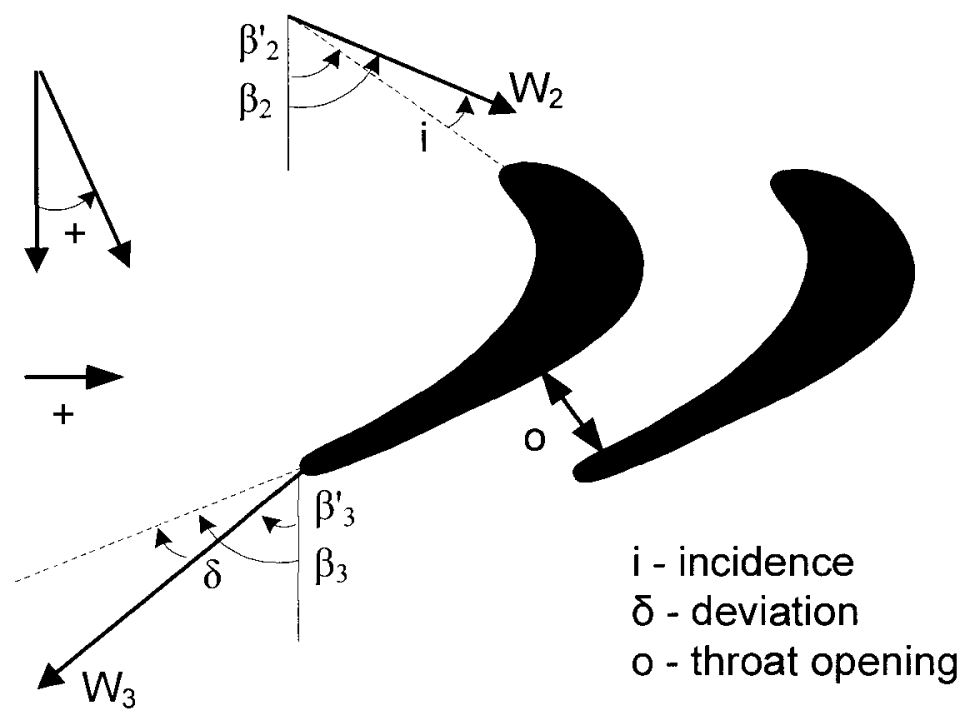

Figure 3.4: Incidence, deviation, and throat opening conventions 
The incidence at design has been correlated empirically against the inlet flow angle by Moustapha et al (2003). The amount of incidence at the design point is given by:

$$
\mathrm{i}=0.175\left(\alpha_{\text {in }}\right)-5.5 \mathrm{deg}
$$

The amount of deviation plays a larger role in the preliminary design of the stage. The deviation is accounted for by using improved correlations for axial turbine rows presented by Zhu and Sjolander (2005). For Mach numbers up to approximately 0.6 the deviation is given as:

$$
\delta=17.3 \cdot \frac{\left(\frac{\mathrm{s}}{\mathrm{c}}\right)^{0.05} \cdot\left(\alpha_{\text {in }}+\alpha_{\text {out }}\right)^{0.63} \cdot \cos ^{2}(\Phi) \cdot(\operatorname{tmax} / \mathrm{c})^{0.29}}{\left[30+0.01 \cdot\left(\alpha_{\text {in }}^{\prime}\right)^{2.07}\right] \cdot \tanh \left(\frac{\operatorname{Re}}{200000}\right)}
$$

For straight-backed blades with Mach numbers in excess of 1, the outlet flow angle is usually considered to be equal to the blade metal angle. The deviation for Mach numbers in the range of $0.6-1.0$ is assumed to vary linearly between the two values.

For use in later calculations, the throat opening (o) may be calculated as a function of the blade metal angle and spacing. This can be seen in Equation 3.52.

$$
\mathrm{o}=\mathrm{s} \cdot \cos \left(\alpha_{\mathrm{ex}}^{\prime}\right)
$$




\subsection{Performance Prediction}

This section will describe the method used for the aerodynamic performance prediction. The method is based largely on that outlined by Kacker and Okapuu (1982). Improved loss coefficients are included where appropriate and referenced therein. The prediction is an integral part of the code as the output is returned to the aerodynamic design to ensure the fluid states at each station are consistent. In order to make an accurate prediction of the overall stage performance, an estimate of the blade trailing edge thickness, and tip clearance are required. Typical values for trailing edge thicknesses depend solely on the manufacturing capability and whether or not blade cooling is employed. For uncooled airfoils a minimum thickness of $0.381 \mathrm{~mm}(0.015 \mathrm{in})$ is recommended. With respect to tip clearances, it is found that modern turbine rotors obtain hot-running tip clearance of the order of 0.5 percent span.

The meanline prediction method used in the design tool is based largely on data obtained from experimental cascades. For this reason, it is first necessary to convert all rotor properties to the relative frame of reference by equations shown previously, 3.213.22. As explained in the literature review, the method is a continuation and improvement of the work of numerous authors. The method was first published by Ainely and Mathieson (1951), refined by Dunham and Came (1970), and modified again by Kacker and Okapuu (1982). Improved profile loss and deviation correlations are also presented by Zhu and Sjolander (2005).

The overall total pressure loss $\left(\mathrm{Y}_{\mathrm{T}}\right)$, expressed in terms of cascade exit dynamic pressure, is assumed to be the sum of profile $\left(\mathrm{Y}_{\mathrm{P}}\right)$, secondary $\left(\mathrm{Y}_{\mathrm{S}}\right)$, trailing edge $\left(\mathrm{Y}_{\mathrm{TET}}\right)$, and tip leakage $\left(\mathrm{Y}_{\mathrm{TC}}\right)$ losses:

$$
\mathrm{Y}_{\mathrm{T}}=\mathrm{Y}_{\mathrm{P}} \cdot \mathrm{f}_{\mathrm{Re}}+\mathrm{Y}_{\mathrm{S}}+\mathrm{Y}_{\mathrm{TET}}+\mathrm{Y}_{\mathrm{TC}}
$$




\subsubsection{Profile Losses}

The basis of the profile loss coefficient is given by the combined work of Ainley and Mathieson (1951) and Dunham and Came (1970), referred to as AMDC. The results are based on the pitch-to-chord ratio (in terms of true chord) and exit flow angle $\alpha_{2}$ for two cases, $\alpha^{\prime}{ }_{1}=0$ and $\alpha^{\prime}{ }_{1}=\alpha_{2}$. The values of the loss coefficients for these special cases are shown in Figures 3.5 (a) and (b). Curve fits for this data are also available in Sjolander (2002), and have been implemented into the code. These curve fits are also given in Appendix $\mathrm{C} 1$. 
(a)

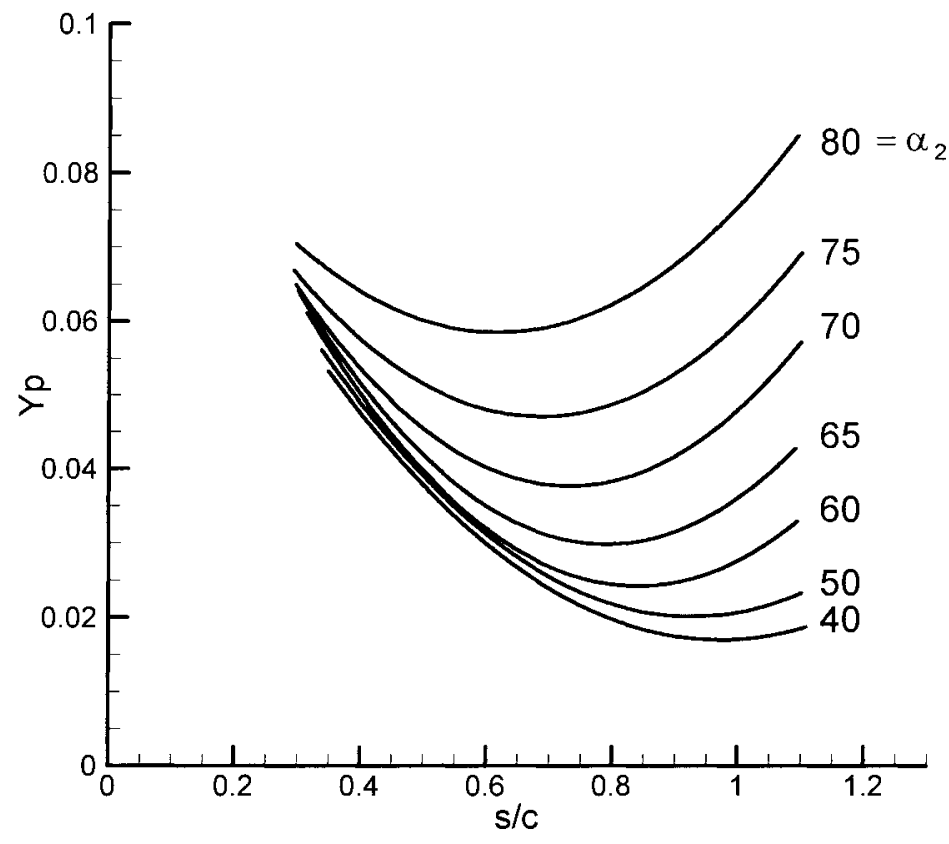

(b)

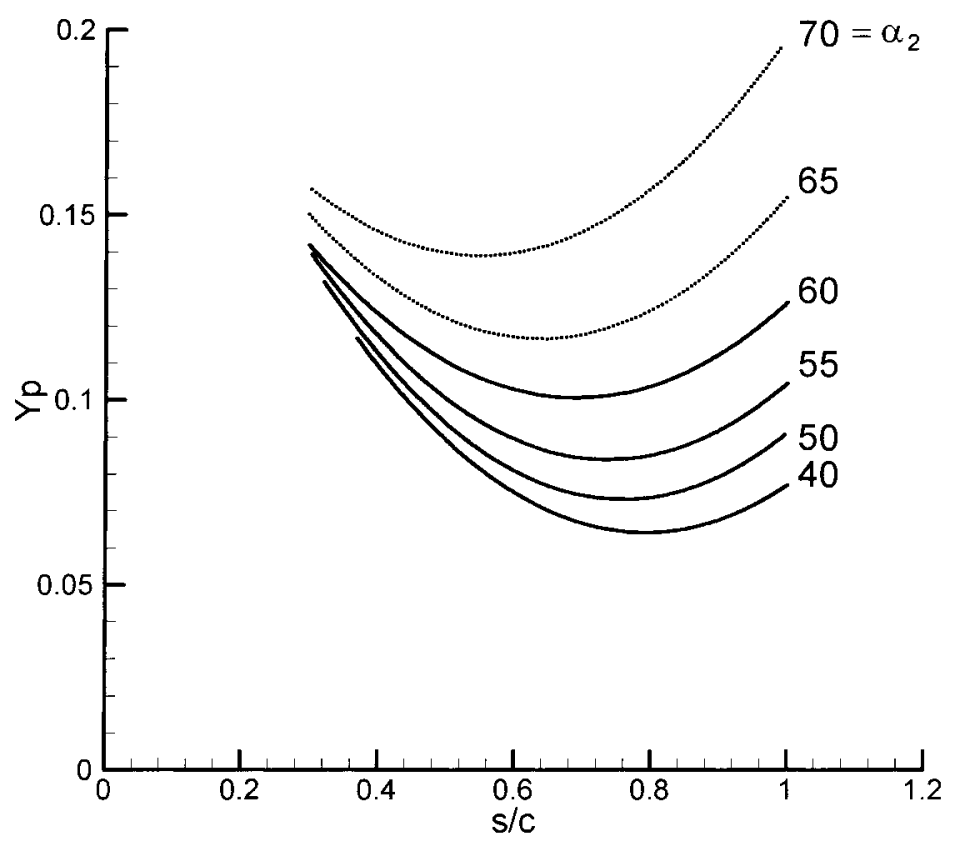

Figure 3.5: (a) Profile loss coefficient for $\alpha^{\prime}{ }_{1}=0$ and (b) Profile loss coefficient for $\alpha_{1}^{\prime}=\alpha_{2}$ 
For any other combination of flow angles, the graphs are interpolated by:

$$
\mathrm{Y}_{\mathrm{P}}^{\prime}=\left\{\mathrm{Y}_{\mathrm{P}\left(\alpha_{1}^{\prime}=0\right)}+\left|\frac{\alpha_{1}^{\prime}}{\alpha_{2}}\right|\left(\frac{\alpha_{1}^{\prime}}{\alpha_{2}}\right) \cdot\left[\mathrm{Y}_{\mathrm{P}\left(\alpha_{1}^{\prime}=\alpha_{2}\right)}-\mathrm{Y}_{\mathrm{P}\left(\alpha_{1}^{\prime}=0\right)}\right]\right\} \cdot\left(\frac{\mathrm{t}_{\max } / \mathrm{c}}{0.2}\right)^{\frac{\alpha_{1}^{\prime}}{\alpha_{2}}}
$$

In the original AMDC loss correlation $\left(\mathrm{Y}_{\mathrm{P}}^{\prime}\right)$, this result was multiplied by a Reynolds number correction factor $\left(f_{\mathrm{Re}}\right)$ and used for the profile loss coefficient. Kacker and Okapuu (1982) suggest the use of additional factors to compensate for the shock losses $\left(Y_{\text {shock }}\right)$ that occur at the blade leading edges, the channel flow acceleration $\left(K_{P}\right)$, and the supersonic drag rise (CFM), if applicable.

Subsonic shock losses can occur at the blade leading edges due to local flow acceleration adjacent to the highly curved leading edges when inlet Mach numbers exceed about 0.4 . The variation of this shock loss can be described by the following equation:

$$
\mathrm{Y}_{\text {shock }}=0.75\left(\mathrm{M}_{1}-0.4\right)^{1.75} \cdot\left(\frac{\mathrm{R}_{\mathrm{h}}}{\mathrm{R}_{\mathrm{t}}}\right) \cdot\left(\frac{\mathrm{P}_{1}}{\mathrm{P}_{2}}\right) \cdot\left[\frac{1-\left(1+\frac{\gamma-1}{2} \mathrm{M}_{1}^{2}\right)^{\frac{\gamma}{\gamma-1}}}{1-\left(1+\frac{\gamma-1}{2} \mathrm{M}_{2}^{2}\right)^{\frac{\gamma}{\gamma-1}}}\right]
$$

The effects of the exit Mach number and channel flow acceleration are then given by:

$$
\mathrm{K}_{\mathrm{P}}=1-\mathrm{K}_{2}\left(1-\mathrm{K}_{1}\right)
$$

where

$$
\mathrm{K}_{1}=1-1.25\left(\mathrm{M}_{2}-0.2\right)
$$

and 


$$
\mathrm{K}_{2}=\left[\frac{\mathrm{M}_{1}}{\mathrm{M}_{2}}\right]^{2}
$$

With modern turbine blade designs, the effects of supersonic exit velocities are a major source of pressure loss. The AMDC loss system incorporates the supersonic drag rise according to:

$$
\mathrm{CFM}=1+60\left(\mathrm{M}_{2}-1\right)^{2}
$$

where CFM is a applied as a multiplier to $\mathrm{Y}_{\mathrm{P}}$, if $\mathrm{M}_{2}$ is larger than unity.

With the exception of the Reynolds number correction, this concludes the estimation of the profile loss coefficient as outlined by Kacker and Okapuu (1982). The loss coefficient is calculated as follows:

$$
Y_{P}=0.914\left(\frac{2}{3} Y_{P}^{\prime} \cdot K_{P}+Y_{\text {shock }}\right) \cdot C F M
$$

This value is then multiplied by a Reynolds number correction factor as indicated in Equation 3.52. More recently however, Zhu and Sjolander (2005) have proposed a modification and improvement to the above profile loss coefficient. In this case, the profile loss coefficient given by the AMDC loss system is modified to include a new term on the exponent, $\mathrm{k}_{\mathrm{m}}$, as follows:

$Y_{P}^{\prime}=\left\{Y_{P\left(\alpha_{1}^{\prime}=0\right)}+\left|\frac{\alpha_{1}^{\prime}}{\alpha_{2}}\right|\left(\frac{\alpha_{1}^{\prime}}{\alpha_{2}}\right) \cdot\left[Y_{P\left(\alpha_{1}^{\prime}=\alpha_{2}\right)}-Y_{P\left(\alpha_{1}^{\prime}=0\right)}\right]\right\} \cdot\left(\frac{\mathrm{t}_{\max } / \mathrm{c}}{0.2}\right)^{\mathrm{k}_{\mathrm{tm}} \frac{\alpha_{1}^{\prime}}{\alpha_{2}}}$

where $k_{\mathrm{m}}=+1$ for $t_{\max } / \mathrm{c}<0.2$

$k_{m}=-1$ for $t_{\max } / c>0.2$ 
Also the $2 / 3$ in Equation 3.60 is replaced by a new term, $k_{n}$, such that:

$$
Y_{P}=0.914\left(k_{n} \cdot Y_{P}^{\prime} \cdot K_{P}+Y_{\text {shock }}\right) \cdot C F M
$$

where $k_{n}=2 / 3$ for reaction blades

$\mathrm{k}_{\mathrm{n}}=0.825$ for axial entry nozzles

This new profile loss correlation is accompanied by a new estimate for the Reynolds number correction factor for Reynolds numbers less than 200,000. The remaining correlations are as proposed by Kacker and Okapuu (1982).

$$
\begin{aligned}
f_{\operatorname{Re}} & =\left(\frac{\operatorname{Re}}{2 \times 10^{5}}\right)^{-0.575} & & \text { for } \operatorname{Re} \leq 2 \times 10^{5} \\
& =1.0 & & \text { for } 2 \times 10^{5}<\operatorname{Re}<10^{6} \\
& =\left(\frac{\operatorname{Re}}{2 \times 10^{5}}\right)^{-0.2} & & \text { for } \operatorname{Re}>10^{6}
\end{aligned}
$$

\subsubsection{Trailing Edge Losses}

The trailing edge loss coefficient is based largely in terms of the trailing edge blockage itself. Kacker and Okapuu (1982) express the loss in terms of an energy coefficient, $\Delta \varphi^{2}$ TET, which is simply a function of the specified trailing edge thickness $\left(\mathfrak{t}_{\mathrm{TET}}\right)$ and throat opening, calculated via Equation 3.52. For axial entry nozzles, this energy coefficient is given as:

$$
\Delta \varphi^{2} \operatorname{TET}_{\left(\alpha_{1}^{\prime}=0\right)}=0.0387 \cdot\left(\frac{\mathrm{t}_{\mathrm{TET}}}{\mathrm{o}}\right)+1.139 \cdot\left(\frac{\mathrm{t}_{\mathrm{TET}}}{\mathrm{o}}\right)^{2}-0.917 \cdot\left(\frac{\mathrm{t}_{\mathrm{TET}}}{\mathrm{o}}\right)^{3}
$$

Likewise, for impulse blades, the energy coefficient is expressed as: 


$$
\Delta \varphi^{2} \operatorname{TET}\left(\alpha_{1}^{\prime}=\alpha_{2}\right)=0.0232 \cdot\left(\frac{\mathrm{t}_{\mathrm{TET}}}{\mathrm{o}}\right)+0.576 \cdot\left(\frac{\mathrm{t}_{\mathrm{TET}}}{\mathrm{o}}\right)^{2}-0.450 \cdot\left(\frac{\mathrm{t}_{\mathrm{TET}}}{\mathrm{o}}\right)^{3}
$$

For other blade profiles, not described by Equations 3.64 and 3.65, the energy coefficient is interpolated by Equation 3.66.

$$
\Delta \varphi^{2} \operatorname{TET}=\left\{\Delta \varphi^{2} \operatorname{TET}_{\left(\alpha_{1}^{\prime}=0\right)}+\left|\frac{\alpha_{1}^{\prime}}{\alpha_{2}}\right|\left(\frac{\alpha_{1}^{\prime}}{\alpha_{2}}\right) \cdot\left[\Delta \varphi^{2} \operatorname{TET}_{\left(\alpha_{1}^{\prime}=\alpha_{2}\right)}-\Delta \varphi^{2} \operatorname{TET}\left(\alpha_{1}^{\prime}=0\right)\right]\right\}
$$

Finally, it is then necessary to convert the kinetic energy loss coefficient to a pressure loss coefficient, $\mathrm{Y}_{\mathrm{TET}}$.

$$
\mathrm{Y}_{\mathrm{TET}}=\frac{\left[1-\frac{\gamma-1}{2} \cdot \mathrm{M}_{2}^{2} \cdot\left(\frac{1}{1-\Delta \varphi^{2} \mathrm{TET}}-1\right)\right]^{\frac{-\gamma}{\gamma-1}}-1}{1-\left(1-\frac{\gamma-1}{2} \cdot \mathrm{M}_{2}^{2}\right)^{\frac{-\gamma}{\gamma-1}}}
$$

It should be noted that the trailing edge pressure loss coefficient is only valid for Mach numbers less than unity. For supersonic exit velocities, the supersonic drag rise factor given in Equation 3.59, is assumed to include the effects of trailing edge losses.

\subsubsection{Secondary Losses}

The calculation of the secondary loss coefficient is also largely based on the AMDC loss system, except for its dependence on the blade aspect ratio. It is calculated as follows:

$$
\mathrm{Y}_{\mathrm{S}}^{\prime}=0.0334 \cdot \mathrm{f}_{\mathrm{AR}} \cdot\left(\frac{\cos \left(\alpha_{\mathrm{ex}}\right)}{\cos \left(\alpha_{\mathrm{in}}^{\prime}\right)}\right) \cdot\left(2 \cdot\left[\tan \left(\alpha_{\mathrm{in}}\right)+\tan \left(\alpha_{\mathrm{ex}}\right)\right]\right)^{2} \cdot \frac{\cos ^{2}\left(\alpha_{\mathrm{ex}}\right)}{\cos \left(\alpha_{\mathrm{m}}\right)}
$$


where

$\alpha_{m}=\arctan \left[\frac{1}{2} \cdot\left(\tan \left(\alpha_{\text {in }}\right)-\tan \left(\alpha_{\text {ex }}\right)\right)\right]$

and as outlined by Kacker and Okapuu (1982):

$$
\begin{aligned}
\mathrm{f}_{\mathrm{AR}} & =\frac{1-0.25 \cdot \sqrt{2-\mathrm{AR}}}{\mathrm{AR}} & \text { for } & \mathrm{AR} \leq 2 \\
& =\frac{1}{\mathrm{AR}} & & \text { for } \mathrm{AR}>2
\end{aligned}
$$

As with profile losses, a subsonic Mach number correction is also applied here. This correlation is given by:

$\mathrm{K}_{\mathrm{S}}=1-\mathrm{K}_{3} \cdot\left(1-\mathrm{K}_{\mathrm{P}}\right)$

where

$\mathrm{K}_{3}=\left(\frac{\mathrm{c} \cdot \cos (\Phi)}{\mathrm{h}}\right)^{2}$

and $\mathrm{K}_{\mathrm{P}}$ is given by Equation 3.56.

The secondary loss coefficient is then given by:

$\mathrm{Y}_{\mathrm{S}}=1.2 \cdot \mathrm{Y}_{\mathrm{S}}^{\prime} \cdot \mathrm{K}_{\mathrm{S}}$ 


\subsubsection{Tip Clearance Losses}

Tip clearance losses are a major source of turbine inefficiency. For unshrouded blade tips, it is generally accepted that 1 percent clearance, based on blade height, constitutes about a 2 percent efficiency drop. According to Kacker and Okapuu (1982), most of the available data on tip clearance losses correlated within $+/-15$ percent of the following expression:

$$
\Delta \eta=0.93 \cdot \eta_{\text {to }} \cdot \frac{k}{h \cdot \cos \left(\alpha_{\mathrm{ex}}\right)} \cdot \frac{\mathrm{r}_{\mathrm{t}}}{\mathrm{r}_{\mathrm{m}}}
$$

where $\eta_{\text {to }}$ is the isentropic efficiency for zero tip clearance and $k$ is the desired tip clearance. To use this correlation in the current performance prediction method, it must be converted to a form of pressure loss coefficient. This is accomplished by assuming zero tip clearance in the first iteration and subsequently adjusting the value for tip clearance loss coefficient, $\mathrm{Y}_{\mathrm{TC}}$, until the overall efficiency converges. This is explained in greater detail in the next section. An additional method for shrouded turbines is described by Kacker and Okapuu (1982).

\subsubsection{Total Pressure Loss Coefficient}

The overall total pressure loss coefficient is given by Equation 3.53. Using the total loss coefficient for the stator section, the pressure loss across the nozzles may be computed as follows:

$$
\Delta \mathrm{P}_{0}=\mathrm{Y}_{\mathrm{T}} \cdot\left(\mathrm{P}_{02}-\mathrm{P}_{2}\right)
$$

Given the total pressure at Station 1, as outlined by the design specifications, the total pressure at Station 2 and percentage pressure loss may be calculated.

$$
\mathrm{P}_{02}=\mathrm{P}_{01}-\Delta \mathrm{P}_{0}
$$




$$
\% \mathrm{PL}=\frac{\Delta \mathrm{P}_{0}}{\mathrm{P}_{01}}
$$

The value of Equation 3.77 is then used as a new estimate for the pressure loss entered earlier in Equation 3.23. Ensuring the use of relative quantities $\left(\mathrm{P}_{\text {orel }}\right)$, the rotor loss calculation for zero tip clearance loss is calculated as follows:

$$
\begin{aligned}
& \Delta P_{0 \text { rel }}=Y_{T} \cdot\left(P_{03 \text { rel }}-P_{3}\right) \\
& P_{03 \text { rel }}=P_{2 \text { rel }}-\Delta P_{0 \text { rel }}
\end{aligned}
$$

The static pressure at Station 3, for zero tip clearance, is then given by:

$$
\mathrm{P}_{3}=\mathrm{P}_{03 \mathrm{rel}} \cdot\left[1+\left(\frac{\gamma-1}{2}\right) \cdot \mathrm{M}_{3 \mathrm{rel}}{ }^{2}\right]^{\frac{-\gamma}{\gamma-1}}
$$

This is then converted to the total pressure in the stationary reference frame for Station 3 using Equation 3.21. The total-to-total isentropic efficiency for zero tip clearance is then calculated by:

$$
\begin{aligned}
& \mathrm{T}_{03}^{\prime}=\mathrm{T}_{02} \cdot\left(\frac{\mathrm{P}_{03}}{\mathrm{P}_{01}}\right)^{\frac{\gamma-1}{\gamma}} \\
& \eta_{\text {to }}=\frac{\mathrm{T}_{03}-\mathrm{T}_{01}}{\mathrm{~T}_{03}^{\prime}-\mathrm{T}_{01}}
\end{aligned}
$$

Using Equation 3.74, an estimate of the efficiency loss due to tip clearances is calculated. 
The stage efficiency is then given by:

$$
\eta=\eta_{\text {to }}-\Delta \eta
$$

Using the corrected efficiency calculated by Equation 3.83, a value for the tip clearance loss coefficient may be estimated. The total rotor pressure loss coefficient is then recalculated for the given tip clearance. Equations 3.78-3.82 are then re-calculated to obtain the overall total pressure loss and efficiency of the stage with the tip clearances accounted for. This is repeated until convergence is obtained. The method is detailed in the flow chart of Appendix A2. 


\subsection{Structural Integrity}

To this point, the focus of the design has been on the aerodynamic aspects of the gas turbine stator and rotor blades. For the purposes of this thesis, the preliminary design of the turbine components was separated into two sections: aerodynamic and structural. Both aspects of the design were included in this thesis. Care must be taken in the selection of the design parameters in order to ensure that the component will not only meet the aerodynamic performance specifications, but also be able to sustain structural integrity during service. With this in mind, the design tool conducts a series of structural evaluations of various components as well as the structural design of the blade attachment and turbine casing.

To estimate of the stresses, fatigue life, and creep limits of the design, specific material properties and empirical correlations must be entered into the design tool by the user. These properties include the density, ultimate tensile strength, Poisson's ratio, and yield strength of the rotor and casing as well as the percent elongation and Young's modulus for the casing. Empirical correlations for fatigue life and creep limits are also required. Evidently, the material selection process will depend on the results of these calculations and thus will be an iterative process until the final material is selected.

\subsubsection{Blade Stress Estimation}

To conduct a blade root stress estimation, the average hub and tip radii must be computed. This is done simply by taking the average of the hub and tip radii as calculated at Stations 2 and 3 of the aerodynamic design. To limit the stresses at the blade root, most blades are tapered from hub to tip. An estimate of the taper $\left(\mathrm{K}_{\text {taper }}\right)$ is given by:

$$
\mathrm{K}_{\text {taper }}=\frac{1+\mathrm{A}_{\text {ratio }}}{3}+\frac{\text { HTR }+\mathrm{A}_{\text {ratio }}}{3 \cdot(1+\mathrm{HTR})}
$$


where the blade profile area is estimated by multiplying the axial component of the chord by the maximum thickness of the blade ( $\left.t_{\text {blade_max }}\right)$, such that:

$$
A_{\text {ratio }}=\frac{c_{x t} \cdot t_{\text {blade_max }} t}{c_{x h} \cdot t_{\text {blade_max }} h}
$$

and HTR is simply the hub-to-tip ratio of the average hub $\left(\mathrm{R}_{\mathrm{h}}\right)$ and tip $\left(\mathrm{R}_{\mathrm{t}}\right)$ radii:

$$
\mathrm{HTR}=\frac{\mathrm{R}_{\mathrm{h}}}{\mathrm{R}_{\mathrm{t}}}
$$

Finally, the blade root stress $\left(\sigma_{\mathrm{H}}\right)$ may be estimated by:

$$
\sigma_{\mathrm{H}}=\mathrm{K}_{\text {taper }} \frac{\rho_{\text {rotor }} \cdot \mathrm{N}^{2}}{2} \cdot\left(\mathrm{R}_{\mathrm{t}}^{2}-\mathrm{R}_{\mathrm{h}}^{2}\right)
$$

where $\rho_{\text {rotor }}$ is the rotor material density and $\mathrm{N}$ is the rotational speed in RPM.

Guidelines for preliminary design purposes are usually given in the form $\mathrm{KAN}^{2}$, where accepted values are given by previous designers which are independent of material density. The average blade root stress may be converted to $\mathrm{KAN}^{2}$ by Equation 3.88 .

$$
\mathrm{KAN}^{2}=\frac{2 \cdot \pi \cdot \sigma_{\mathrm{H}}}{\rho_{\text {rotor }}}
$$

\subsubsection{Creep Life}

In order to perform a creep life evaluation, additional user input is required. An estimate of the maximum blade stress is given by Equation 3.87, and an estimate of the maximum blade temperature may be calculated as follows: 


$$
\mathrm{T}_{02 \mathrm{rel}}=\mathrm{T}_{02}-\frac{\mathrm{C}_{2}{ }^{2}}{2 \cdot \mathrm{C}_{\mathrm{p}}}+\frac{\mathrm{W}_{2}{ }^{2}}{2 \cdot \mathrm{C}_{\mathrm{p}}}
$$

where $\mathrm{C}_{2}$ and $\mathrm{W}_{2}$ are the absolute and relative flow velocities calculated previously. For the calculated root stress the user must specify the Larsen-Miller parameter for an appropriate material. This parameter is readily available in any material property handbook or online material supplier. The method simply relates the time to rupture $(\mathrm{t})$ of a material specimen to the applied stress and temperature.

$$
t=10^{\left(\frac{L M \cdot 1000}{T_{02 \text { rel }}}-20\right)}
$$

where LM is the Larsen-Miller parameter entered by the user for the appropriate stress level and material.

\subsubsection{Fatigue Life}

For preliminary design and analysis, an estimate of the actual fatigue life is difficult to predict since it is strongly dependent on the actual component. This requires a substantial amount of testing to be conducted on the component once it has been fabricated. Typically, engineers rely on empirical data that relate the stress of the component to the number of cycles before failure. Additionally, the combined effects of constant and oscillating stresses are important in the design of gas turbine blades. Material data for this type of stress state is readily available in the form of Gerber diagrams, which ensure the designer that the magnitude of both stresses lie within the

appropriate bounds of the material. An example of this will follow in the design section of Chapter 4.

With this in mind, the design tool uses a method proposed by Saravanmuttoo et al. (2001) to estimate the gas bending (or oscillating) stresses on the turbine blade. By combining the effects of this oscillating stress and the constant root stress calculated 
previously, the designer can ensure the blade structure will not fail by fatigue within the desired life. According to Saravanamuttoo et al. (2001), the maximum gas bending stress $\left(\sigma_{\mathrm{gb}}\right)$ may be given by:

$$
\sigma_{\mathrm{gb}}=\mathrm{m}_{\mathrm{air}} \cdot \frac{\mathrm{C}_{02}+\mathrm{C}_{03}}{\mathrm{n}} \cdot \frac{1}{2} \cdot \frac{1}{\mathrm{z}}
$$

where $\mathrm{z}$ is given by:

$$
\mathrm{z}=\frac{1}{\mathrm{~B}} \cdot(10 \cdot \operatorname{tmax} / \mathrm{c})^{\mathrm{n}^{\prime}} \cdot \mathrm{c}^{3}
$$

and $\mathrm{B}$ and $\mathrm{n}^{\prime}$ are given as correlations of blade camber angle $\left(\beta_{\text {camber }}\right)$.

$$
\begin{aligned}
& \mathrm{B}=-8.4375 \cdot \beta_{\text {camber }}+1317.5 \\
& \mathrm{n}^{\prime}=-0.00875 \cdot \beta_{\text {camber }}+1.137 \\
& \beta_{\text {camber }}=\beta_{\mathrm{h} 2}+\beta_{\mathrm{h} 3}
\end{aligned}
$$

The designer must now ensure that the combined effect of the gas bending stress and constant root stress does not exceed that illustrated by the Gerber diagram of a particular material.

\subsubsection{Disk Stress Estimation}

A disk stress estimation is conducted by dividing the disk into a number of radial segments and calculating the radial and tangential stresses at each location. In order to superimpose the rim stress created by the blades, it must be estimated from the hub stress calculated earlier. This is done by determining the force $\left(\mathrm{F}_{\text {blade }}\right)$ created by each blade 
and applying it over the entire disk rim. This is achieved by estimating the blade profile hub area and multiplying it by the hub stress calculated previously as follows:

$$
\mathrm{F}_{\text {blade }}=\sigma_{\mathrm{H}} \cdot\left(\mathrm{c}_{\mathrm{h}} \cdot \mathrm{t}_{\text {blade_max }}\right)
$$

The total force $\left(\mathrm{F}_{\text {total }}\right)$ and rim stress $\left(\sigma_{\text {rim }}\right)$ is then calculated by:

$$
\begin{aligned}
& \mathrm{F}_{\text {total }}=\mathrm{F}_{\text {blade }} \cdot \mathrm{n} \\
& \sigma_{\text {rim }}=\frac{\mathrm{F}_{\text {total }}}{2 \cdot \pi \cdot \mathrm{R}_{\mathrm{h}} \cdot \mathrm{c}_{\mathrm{h}}}
\end{aligned}
$$

The design tool then calculates the radial $\left(\sigma_{\mathrm{r}}\right)$, tangential $\left(\sigma_{\mathrm{t}}\right)$, and resulting von Mises $\left(\sigma_{\mathrm{vM}}\right)$ stresses via:

$$
\begin{aligned}
& \sigma_{\mathrm{r}}=\frac{3+\mathrm{v}}{8} \cdot\left(\mathrm{R}_{\mathrm{h}}-\mathrm{r}\right) \cdot\left(\rho_{\text {rotor }} \cdot \omega^{2}\right)+\sigma_{\text {rim }} \\
& \sigma_{\mathrm{t}}=\frac{1}{8} \cdot\left[(3+v) \mathrm{R}_{\mathrm{h}}{ }^{2}-(1+3 v) \mathrm{r}^{2}\right] \cdot\left(\rho_{\text {rotor }} \cdot \omega^{2}\right)+\sigma_{\text {rim }}
\end{aligned}
$$

where $v$ is Poisson's ratio.

$$
\sigma_{v M}=\sqrt{\sigma_{r}^{2}+\sigma_{t}^{2}-\sigma_{r} \cdot \sigma_{t}}
$$

\subsubsection{Blade Attachment Sizing}

Using the method outlined by Sawyer (1985), a preliminary fir-tree sizing is conducted. The detailed design and analysis is far beyond the scope of this thesis and 
would be required if fir-tree attachments were to be used in the final design. Based on experience, Sawyer (1985) presents a method in which two parameters govern an acceptable design, shown in Figure 3.6.

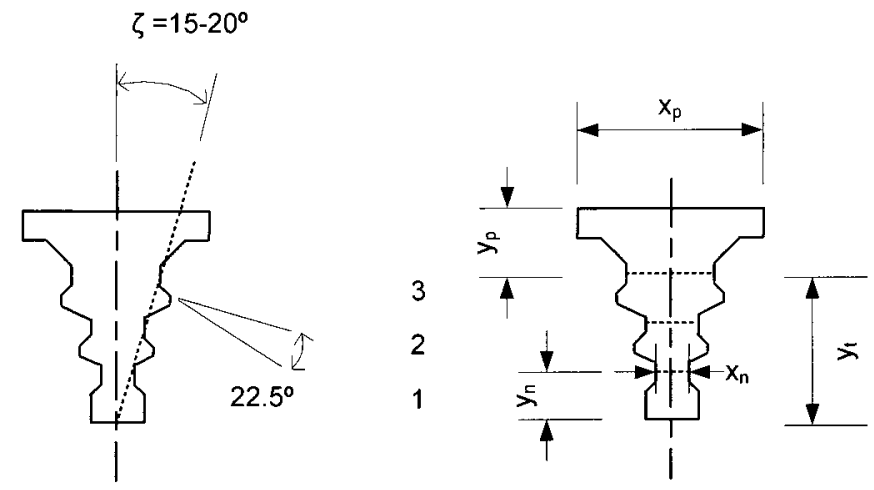

Figure 3.6: Accepted values and nomenclature for fir-tree design

The designer is required to select a value for the blade attachment angle $(\zeta)$ within an acceptable range; whereas, the other angle $\left(22.5^{\circ}\right)$ has been set based on experience. Using these accepted values with an assumed platform $\left(\mathrm{y}_{\mathrm{p}}\right)$ and fir-tree depth $\left(\mathrm{y}_{\mathrm{t}}\right)$, the geometry of the blade attachment may be calculated. The platform width $\left(\mathrm{x}_{\mathrm{p}}\right)$ and height $\left(\mathrm{y}_{\mathrm{p}}\right)$ may be estimated by:

$$
\begin{aligned}
& \mathrm{x}_{\mathrm{p}}=\frac{2 \pi \mathrm{R}_{\mathrm{h}}}{\mathrm{n}_{\mathrm{R}}} \\
& \mathrm{y}_{\mathrm{p}}=0.25 \cdot \mathrm{h}_{\mathrm{R}}
\end{aligned}
$$

where $h_{R}$ has been previously defined as the rotor blade height. The total depth of the firtree can then be estimated by Equation 3.104.

$$
\mathrm{y}_{\mathrm{t}}=0.5 \cdot \mathrm{h}_{\mathrm{R}}
$$

By assuming three teeth, the height of each fir-tree segment is then: 


$$
\Delta y=\frac{y_{t}}{3}
$$

Any number of teeth can be assumed at this point. Most often 3 to 5 teeth are used in gas turbine design. The number of teeth will be quantified by the structural evaluation of the blade attachment.

Based on the above assumptions, $y_{1}, y_{2}$ and $y_{3}$ are determined by:

$$
\begin{aligned}
& \mathrm{y}_{1}=\Delta \mathrm{y} \\
& \mathrm{y}_{2}=\mathrm{y}_{1}+\Delta \mathrm{y} \\
& \mathrm{y}_{3}=\mathrm{y}_{2}+\Delta \mathrm{y}
\end{aligned}
$$

The values for each corresponding value $\mathrm{x}_{\mathrm{n}}$ are then determined via Equation 3.107.

$$
\mathrm{x}_{\mathrm{n}}=2 \cdot\left(\mathrm{y}_{\mathrm{n}} \cdot \tan (\zeta)\right)
$$

\subsubsection{Burst Speed Estimation}

An estimate of the rotor burst speed is provided by Sawyer (1985). Two approaches are provided; the first is based on the reasoning of Roark and Young (1975), and the other, a conservative approach, based on the yield limit of the material. According to Roark and Young (1975), the elastic centrifugal stress is linearly proportional to $\rho \omega^{2} \mathrm{r}^{2}$ at any given location in a rotating structure. Also, at the point of disk burst, any ductile material will strain harden, thus the stress will be higher than the initial yield stress and be equal to the ultimate strength of the material. Therefore, Roark's criterion for disk burst is given by:

$$
\sigma_{\mathrm{UTS}}=\frac{1}{3} \cdot \rho_{\text {rotor }} \cdot \omega^{2} \cdot \mathbf{r}^{2}
$$


where a factor of $1 / 3$ is used as a proportionality constant at the area of peak stress. Rearranging the above equation, it is possible to determine the minimum speed at which disk burst ( $\left.\omega_{\text {burst }}\right)$ may occur:

$$
\omega_{\text {burst }}=\sqrt{\frac{3 \cdot \sigma_{\text {UTS }}}{\rho_{\text {rotor }} \cdot r_{m}^{2}}}
$$

The second approach is based on the maximum permissible stress before the material reaches its yield strength. This method will always yield a conservative approach. The value is calculated by determining the value for $\omega$ at $r=0$ for Equation 3.100, such that $\sigma_{t}$ is equal to the yield stress of the material. For safety considerations, the lower value of burst speed is taken.

\subsubsection{Casing Thickness Evaluation}

The final step carried out by the design tool is the calculation of the minimum casing thickness required to contain a tri-hub disk burst. This is such that the thickness of the casing should be sufficient to contain the kinetic energy of one third of the rotor at the burst speed calculated previously. The thickness calculation is based on a procedure outlined by Hagg and Sankey (1973). The first step in the determination of the minimum thickness is the evaluation of the dynamic material properties of the casing material. These properties typically differ quite significantly from the static values, and they are typically not published in material handbooks as they are usually design specific. In this evaluation, the dynamic strength $\left(\sigma_{\mathrm{d}}\right)$ and dynamic shear strength $\left(\tau_{\mathrm{d}}\right)$ are given by Hagg and Sankey (1973) as:

$$
\begin{aligned}
& \sigma_{\mathrm{d}}=1.25 \cdot \sigma_{\mathrm{UTS}} \\
& \mathrm{K} \tau_{\mathrm{d}}=0.27 \cdot \sigma_{\mathrm{d}}
\end{aligned}
$$


where $\mathrm{K}$ is an empirical constant used to adjust the calculations and test results of Hagg and Sankey (1973).

The next step is to determine the mass of one third of the rotor (M1) and its kinetic energy $\left(\mathrm{KE}_{\mathrm{R}}\right)$ at burst. This is calculated as follows:

$$
\begin{aligned}
& \mathrm{M} 1=\frac{\pi \cdot \mathrm{r}_{\mathrm{m}}^{2}}{3} \cdot \mathrm{c}_{\mathrm{Rhx}} \cdot \rho_{\text {rotor }} \\
& \mathrm{r}_{\text {fragment }}=\frac{2}{3} \cdot \mathrm{r}_{\mathrm{m}} \cdot \frac{\sin (\chi / 2)}{\chi / 2}
\end{aligned}
$$

where $r_{\text {fragment }}$ is the radial distance to the center of gravity of the fragment and $\chi$ is the fragment angle (120 degrees for a one third rotor containment design). The fragment velocity $\left(\mathrm{V}_{\text {fragment }}\right)$ and kinetic energy are then given by:

$$
\begin{aligned}
& \mathrm{V}_{\text {fragment }}=\mathrm{r}_{\text {fragment }} \cdot \omega_{\text {burst }} \\
& \mathrm{KE}_{\mathrm{R}}=\frac{1}{2} \cdot \mathrm{Ml} \cdot \mathrm{V}_{\text {fragment }}{ }^{2}
\end{aligned}
$$

The containment analysis is detailed as a two stage process. Stage 1 of the process is confined to compression of the material over the impact area $\left(\mathrm{A}_{\text {impact }}\right)$ and shearing of the material over the perimeter of the impact area. The energy $\left(E_{c}\right)$ associated with the compression of the material is related to the impact area as follows:

$$
\mathrm{E}_{\mathrm{c}}=\mathrm{A}_{\text {impact }} \cdot \mathrm{t}_{\text {casingl }} \cdot \varepsilon_{\mathrm{c}} \cdot \sigma_{\mathrm{d}}
$$

where $A$ is the impact area of the casing, $t_{\text {casing1 }}$ is the casing thickness for Stage 1 containment, and $\varepsilon_{\mathrm{c}}$ is the compressive strain. In the method of Hagg and Sankey (1973), 
a relative low average value of $\varepsilon_{\mathrm{c}}=0.07$ was used in all calculations. The impact area may be estimated as follows:

$$
\mathrm{A}_{\text {impact }}=\frac{2 \cdot \pi \cdot \mathrm{R}_{\mathrm{t}}}{3} \cdot \mathrm{c}_{\mathrm{Rhx}}
$$

The energy associated with the shear $\left(E_{s}\right)$ of the material is then expressed as:

$$
\mathrm{E}_{\mathrm{s}}=\mathrm{K} \tau_{\mathrm{d}} \cdot \mathrm{P}_{\text {shear }} \cdot \mathrm{t}_{\text {casingl }}{ }^{2}
$$

where $\mathrm{P}_{\text {shear }}$ is the perimeter of the impact area and may be estimated as:

$$
P_{\text {shear }}=2 \cdot\left(\frac{2 \cdot \pi \cdot R_{t}}{3}\right)+2 \cdot c_{R h x}
$$

The next step in the process is to determine a suitable estimate for the mass of the casing which will undergo compression and shear during the impact process. This estimation can have a significant effect on the casing thickness as the material adjacent to the impact area will also provide some resistance to perforation by the rotor disk. The conservative approach is to take the volume immediately in line of the impact area. Thus, the casing mass $\left(\mathrm{M}_{2}\right)$ resisting perforation may be estimated as follows:

$$
\mathrm{M} 2=\frac{2 \cdot \pi \cdot \mathrm{R}_{\mathrm{t}}}{3} \cdot \mathrm{c}_{\mathrm{Rhx}} \cdot \rho_{\text {casing }} \cdot \mathrm{t}_{\text {casing } 1}
$$

For Stage 1, the energy dissipated in the momentum transfer due to impact is given as:

$$
\Delta \mathrm{E}_{1}=\mathrm{KE}_{\mathrm{R}} \cdot\left(1-\frac{\mathrm{M} 1}{\mathrm{M} 1+\mathrm{M} 2}\right)
$$


Therefore, the minimum casing thickness for Stage 1 containment can be calculated by ensuring that the following inequality holds true:

$$
\mathrm{E}_{\mathrm{s}}+\mathrm{E}_{\mathrm{c}}>\Delta \mathrm{E}_{1}
$$

This is done by substituting Equations 3.116, 3.118 and 3.121 into 3.122 and solving for $\mathrm{t}_{\text {casing1. }}$. The resulting equation is given by:

$$
\mathrm{K} \tau_{\mathrm{d}} \cdot \mathrm{P}_{\text {shear }} \cdot \mathrm{t}_{\text {casing } 1}{ }^{2}+\mathrm{A}_{\text {impact }} \cdot \mathrm{t}_{\text {casingl }} \cdot \varepsilon_{\mathrm{c}} \cdot \sigma_{\mathrm{d}}>\mathrm{KE}_{\mathrm{R}} \cdot\left(1-\frac{\mathrm{M} 1}{\mathrm{M} 1+\mathrm{M} 2}\right)
$$

If perforation does not occur in Stage 1, the process enters Stage 2. In this stage, the residual fraction, $\Delta \mathrm{E}_{2}$, of the initial kinetic energy of the fragment is considered. In Stage 2, energy is dissipated by biaxial tension strain, or stretching. The energy associated with this process can be given by:

$$
\mathrm{E}_{\mathrm{t}}=\mathrm{Q}_{\mathrm{mat}} \cdot \varepsilon_{\mathrm{f}} \cdot \sigma_{\mathrm{d}}
$$

where $\mathrm{Q}_{\mathrm{mat}}$ is the volume of shell material that is strained an allowable amount equal to $\varepsilon_{\mathrm{f}}$ (percent elongation to fracture of the casing material). An estimate for $\mathrm{Q}_{\mathrm{mat}}$ is given by:

$$
\mathrm{Q}_{\text {mat }}=3 \cdot \mathrm{P}_{\text {shear }} \cdot \mathrm{t}_{\text {casing } 2}{ }^{2}+\mathrm{A}_{\text {impact }} \cdot \mathrm{t}_{\text {casing2 }}
$$

The criterion for containment in Stage 2 is then given by:

$$
\mathrm{E}_{\mathrm{t}}>\Delta \mathrm{E}_{2}
$$

where 


$$
\Delta \mathrm{E}_{2}=\mathrm{KE}_{\text {rotor }} \cdot\left(\frac{\mathrm{M} 1}{\mathrm{M} 1+\mathrm{M} 2}\right)
$$

Using Equation 3.126, an estimate for the casing thickness based on the Stage 2 criterion for containment is calculated and compared to that obtained for Stage 1. To ensure containment of the rotor segments at burst the larger of these values is selected and used for the preliminary design. This approach does not explicitly give any allowable safety factor. A safety factor may be added to this approach by adding a multiplier to the kinetic energy term calculated in Equation 3.115. An inherent safety factor is included in the design as a result of the casing layout. This will be presented in a later section. Due to the design decisions taken for these components, the added turbomachinery casing provides additional material for containment. 


\subsection{Design Tool Summary}

At the end of the design tool algorithm, a summary of the important geometry, fluid states, and performance expectations is presented for the use of the designer. The summary contains the most useful results calculated within the design tool. A list of these results is presented in Tables 3.2 and 3.3. Table 3.2 includes a summary of flow conditions which is outputted for each of the three stations as well as the resulting performance expectations. Table 3.3 presents a list of the required geometrical data used for creating the solid and computational models. This data should be sufficient to adequately model the geometry for further aerodynamic or structural evaluation. As can be seen in the preceding chapter, a number of intermediate steps are required to obtain the overall blade geometry and performance estimation. The results of these steps may also be required for future use. These results are summarized in Appendix B2.

\begin{tabular}{|c|c|}
\hline $\mathrm{P}$ & Static pressure \\
\hline $\mathrm{P}_{0}$ & Total pressure \\
\hline $\mathrm{T}_{0}$ & Total temperature \\
\hline $\mathrm{M}$ & Mach number \\
\hline $\mathrm{M}_{\mathrm{rel}}$ & Relative Mach number \\
\hline $\mathrm{C}_{\mathrm{x}}$ & Axial velocity \\
\hline $\mathrm{C}_{\theta}$ & Circumferential velocity \\
\hline $\mathrm{C}$ & Velocity \\
\hline$\alpha$ & Absolute flow angle \\
\hline $\mathrm{W}$ & Relative velocity \\
\hline$\beta$ & Relative flow angle \\
\hline
\end{tabular}

\begin{tabular}{|c|c|}
\hline$\eta$ & Total-to-total isentropic efficiency \\
\hline$\Delta \mathrm{h}_{0}$ & Specific work output \\
\hline
\end{tabular}

\begin{tabular}{|c|c|}
\hline KAN $^{2}$ & Area-speed ratio \\
\hline$\sigma_{\mathrm{H}}$ & Blade root stress \\
\hline
\end{tabular}

Table 3.2: Output station flow conditions and performance expectations 


\begin{tabular}{|c|c|c|c|c|}
\hline \multirow{3}{*}{$\frac{\vec{\Phi}}{\underline{\underline{\Xi}}}$} & $\mathrm{r}_{\mathrm{h}}$ & Hub radius & $\alpha_{h}^{\prime}$ & Metal angle (hub) \\
\hline & $r_{m}$ & Meanline radius & $\alpha_{\mathrm{m}}^{\prime}$ & Metal angle (meanline) \\
\hline & $r_{t}$ & Tip radius & $\alpha_{\mathrm{t}}^{\prime}$ & Metal angle (tip) \\
\hline
\end{tabular}

\begin{tabular}{|c|c|c|c|c|}
\hline \multirow{3}{*}{ 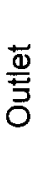 } & $\mathrm{r}_{\mathrm{h}}$ & Hub radius & $\alpha_{h}^{\prime}$ & Metal angle (hub) \\
\hline & $\mathrm{r}_{\mathrm{m}}$ & Meanline radius & $\alpha_{\mathrm{m}}^{\prime}$ & Metal angle (meanline) \\
\hline & $\mathrm{r}_{\mathrm{t}}$ & Tip radius & $\alpha_{\mathrm{t}}^{\prime}$ & Metal angle (tip) \\
\hline
\end{tabular}

\begin{tabular}{|c|c|}
\hline$c_{\mathrm{Nhx}}$ & Axial chord (hub) \\
\hline$c_{\mathrm{Nmx}}$ & Axial chord (meanline) \\
\hline$c_{\mathrm{Ntx}}$ & Axial chord (tip) \\
\hline
\end{tabular}

\begin{tabular}{|c|c|}
\hline$c_{\mathrm{N}}$ & True chord \\
\hline $\mathbf{t}_{\mathrm{max}} / \mathrm{c}_{\mathrm{N}}$ & Thickness-to-chord ratio \\
\hline $\mathrm{n}_{\mathrm{N}}$ & Number of stator blades \\
\hline$\Phi_{\mathrm{N}}$ & Stagger angle \\
\hline
\end{tabular}

ROTOR BLADES

\begin{tabular}{|c|c|c|c|c|}
\hline & $r_{\mathrm{h}}$ & Hub radius & $\beta_{\mathrm{h}}^{\prime}$ & Metal angle (hub) \\
\hline \multirow{\Phi}{\Xi}{} & $r_{\mathrm{m}}$ & Meanline radius & $\beta_{\mathrm{m}}^{\prime}$ & Metal angle (meanline) \\
\hline $\mathrm{r}_{\mathrm{t}}$ & Tip radius & $\beta_{\mathrm{t}}^{\prime}$ & Metal angle (tip) \\
\hline
\end{tabular}

\begin{tabular}{|c|c|c|c|c|}
\hline \multirow{3}{*}{$\frac{\vec{\Phi}}{\bar{\Xi}}$} & $\mathrm{r}_{\mathrm{h}}$ & Hub radius & $\beta_{\mathrm{h}}^{\prime}$ & Metal angle (hub) \\
\hline & $\mathrm{r}_{\mathrm{m}}$ & Meanline radius & $\beta_{m}^{\prime}$ & Metal angle (meanline) \\
\hline & $\mathrm{r}_{\mathrm{t}}$ & Tip radius & $\beta_{t}^{\prime}$ & Metal angle (tip) \\
\hline
\end{tabular}

\begin{tabular}{|c|c|}
\hline$c_{\mathrm{Nhx}}$ & Axial chord (hub) \\
\hline $\mathrm{c}_{\mathrm{Nmx}}$ & Axial chord (meanline) \\
\hline $\mathrm{c}_{\mathrm{Ntx}}$ & Axial chord (tip) \\
\hline
\end{tabular}

\begin{tabular}{|c|c|}
\hline$c_{R}$ & True chord \\
\hline$t_{\text {max }} / c_{R}$ & Thickness-to-chord ratio \\
\hline $\mathrm{n}_{\mathrm{R}}$ & Number of rotor blades \\
\hline$\Phi_{\mathrm{R}}$ & Stagger angle \\
\hline
\end{tabular}

Table 3.3: Geometry output summary 
This concludes the description of the underlying equations and methods applied in the preliminary design tool. Two examples of its use will be presented in the following chapter in the design of a single stage gas generator turbine and single stage power turbine. The output of the design tool is then verified both aerodynamically and structurally using state-of-the-art software tools for computational fluid dynamics and finite element methods. These results will be found in subsequent chapters. 


\section{Chapter 4}

\section{Gas Generator and Power Turbine Design}

The purpose of this chapter is to describe the design of two axial flow turbines which were developed using the design tool explained in the previous chapter. The output of the design tool was used to lay out the blade profiles for complete threedimensional aerodynamic evaluation using computational fluid dynamics. Once a satisfactory aerodynamic performance was obtained, the design was evaluated structurally. This chapter will describe the outputs from the design tool as calculated. Validation and verification of these results will be presented in subsequent chapters.

Section 4.1 will illustrate the design of a single stage gas generator turbine for use in the 1-MW gas turbine engine previously described. Following this, Section 4.2 will summarize the design of a single stage power turbine for use downstream of the gas generator in the same 1-MW engine. The chapter will also include a summary of the preliminary mechanical design of the turbine casings required for the engine. 


\subsection{Gas Generator Turbine Design}

This section will present the overall design of the gas generator turbine components. The design specifications, preliminary design decisions, as well as the actual design, and performance predictions will be presented.

\subsubsection{Design Specifications}

The gas generator turbine has been specified to produce a total power output of approximately $1600 \mathrm{~kW}$ with a mass flow rate of $5.5 \mathrm{~kg} / \mathrm{s}$. This is the power required to drive the 8:1 pressure ratio compressor at the engine's design point with an allowance for the mechanical inefficiency of the shaft. The specified total temperature and pressure at the turbine inlet are $1200 \mathrm{~K}$ and $763,000 \mathrm{~Pa}$, respectively. It was also required that the total-to-total polytropic stage efficiency be no less than 88 percent $(89.4$ percent isentropic). Structurally, all engine components must maintain full structural integrity and tolerate $500 \mathrm{start} / \mathrm{stop}$ cycles at 4 hours each for a total of 2000 hours.

The first step in the design process was to determine an appropriate rotational speed of the gas generator rotor. This value is typically limited by the compressor design and thus a value of 35,000 RPM was chosen. The selection of the gas generator speed is based on specific speed correlations as well as tradeoffs conducted between the compressor and turbine designers. Based on the non-dimensional specific speed of 0.703 , the turbine rotor could be of the axial or radial configuration. An axial configuration was selected.

\subsubsection{Inputs}

As mentioned in Chapter 3, a selected number of design parameters must be assigned values at the beginning of the program. These values must be determined based on judicious selection of the parameters governed by the nature of the design. For a single stage high pressure ratio design, the selection of the stage loading and flow 
coefficients will have a major impact on the stage performance. Using the Smith chart, the stage loading and flow coefficient were chosen to be 1.44 and 0.56 , respectively. The reaction was set slightly below 0.5 to limit the amount of swirl in the exit plane of the rotor. A value of 0.4 was selected to ensure the exit swirl angle was less than 15 degrees in order to limit the losses in the ducts between the gas generator and power turbines. For the gas generator stage, the inlet flow angle is assumed to be 0 degrees. For both designs, a value of 0.98 was chosen for the blockage factor. This value indicates that approximately 2 percent of the geometrical area is blocked by the blade trailing edges and boundary layers. To calculate the number of blades, the blade or Zweifel loading coefficient is specified. Based on the nature of the design, a relatively modest value of 1.1 was chosen. Values in excess of this may be used to reduce the number of blades; however, it is unnecessary for this application since the overall engine weight has very little bearing on the engine design. Finally, the aspect ratios of both the stator and rotor blade rows are selected and entered by the designer. For the gas generator stator blades, a value of 0.5 was selected. Although it is recommended to avoid selecting values less than 1, numerous designs have obtained desirable efficiencies in this range; see Kacker and Okapuu (1982).

\subsubsection{Blade Design}

With the design parameters entered, the program calculates the various flow and metal angles for 3 sections: the hub, meanline, and tip. The hub and tip radii are estimated, and additional calculations are conducted for the fluid states and Mach numbers at each station. For the given design parameters, a total-to-total isentropic stage efficiency is estimated to be 86 percent. Although this value is below the target efficiency, it is still deemed satisfactory for the given design conditions. Also, the blade rows have yet to be optimized and further work is planned. The meanline design velocity triangles at a radius of $123 \mathrm{~mm}$ are shown in Figure 4.1. One may notice the change in axial velocity at Station 2. Typically during the preliminary design phase of a gas turbine stage, the axial component of velocity remains constant throughout the stage. Due to the nature of this high-pressure ratio design, a large annular flare would be required to permit 
a constant axial velocity. This, in turn, causes a large variation in the blade tip radius of the rotor from the leading to trailing edges. This is satisfactory for the preliminary design phase; however, in actual operation this will yield uncontrollable tip clearance issues, and it will prove difficult to design. It has been advised to maintain a constant blade tip radius in the design of the rotor. For this reason, the axial velocity component is reduced in the design tool to more accurately depict the flow conditions that will occur during operation of the engine.

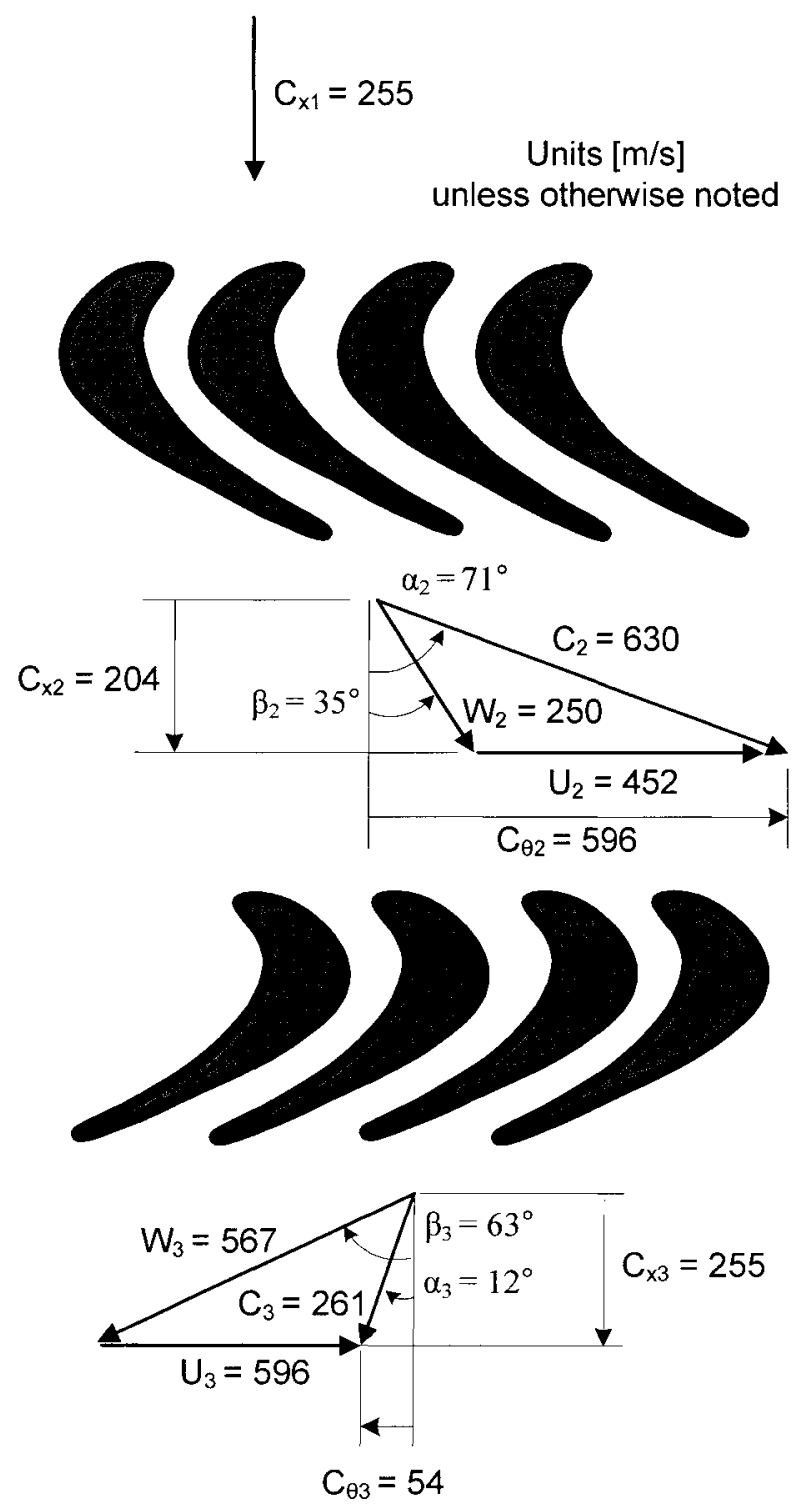

Figure 4.1: Gas generator turbine meanline velocity triangles 
It has been estimated that approximately 19 stator blades and 41 rotor blades are required to efficiently expand the flow and produce the necessary work output of the stage. These values will be used for preliminary design purposes but may be changed slightly for vibrational or structural concerns, to be described later. A summary of the fluid properties and the Mach numbers at each station are shown in Table 4.1.

\begin{tabular}{|c|c|c|c|}
\cline { 2 - 4 } \multicolumn{1}{c|}{} & Station 1 & Station 2 & Station 3 \\
\hline $\mathrm{P}_{0}[\mathrm{~Pa}]$ & 763000 & 720300 & 243800 \\
\hline $\mathrm{T}_{0}[\mathrm{~K}]$ & 1200 & 1200 & 945 \\
\hline $\mathrm{P}[\mathrm{Pa}]$ & 693000 & 386200 & 214400 \\
\hline
\end{tabular}

\begin{tabular}{|c|c|c|c|}
\hline Hub & \multicolumn{3}{|c|}{} \\
\hline $\mathrm{M}$ & 0.38 & 1.14 & 0.45 \\
\hline $\mathrm{M}_{\text {rel }}$ & & 0.67 & 0.88 \\
\hline
\end{tabular}

\begin{tabular}{|c|c|c|c|}
\hline Meanline & \multicolumn{3}{|l|}{} \\
\hline $\mathrm{M}$ & 0.38 & 1.01 & 0.44 \\
\hline $\mathrm{M}_{\mathrm{rel}}$ & & 0.40 & 0.96 \\
\hline
\end{tabular}

\begin{tabular}{|c|c|c|c|}
\hline Tip & \multicolumn{3}{|c|}{} \\
\hline $\mathrm{M}$ & 0.38 & 0.91 & 0.44 \\
\hline $\mathrm{M}_{\text {rel }}$ & & 0.41 & 1.04 \\
\hline
\end{tabular}

Table 4.1: Summary of gas generator turbine fluid states

\subsubsection{Aerodynamic Performance}

A total isentropic stage efficiency of 86 percent has been estimated by the performance evaluation described in Chapter 3. By today's standards, this efficiency may be regarded as quite low for a given turbine stage; however, it still serves as a good starting point for the preliminary design. A large portion of this inefficiency can be attributed to the pressure loss occurring in the stators. Here, a total pressure loss of 5.5 percent has been estimated. This loss is significantly large and may be reduced by increasing the stator aspect ratio in order to reduce the secondary flow losses. Figure 4.2 
shows a comparison between the different loss mechanisms occurring in both the rotor and the stator. It should be noted that trailing edge losses are assumed to be included in the profile loss coefficient when the exit Mach number exceeds unity.

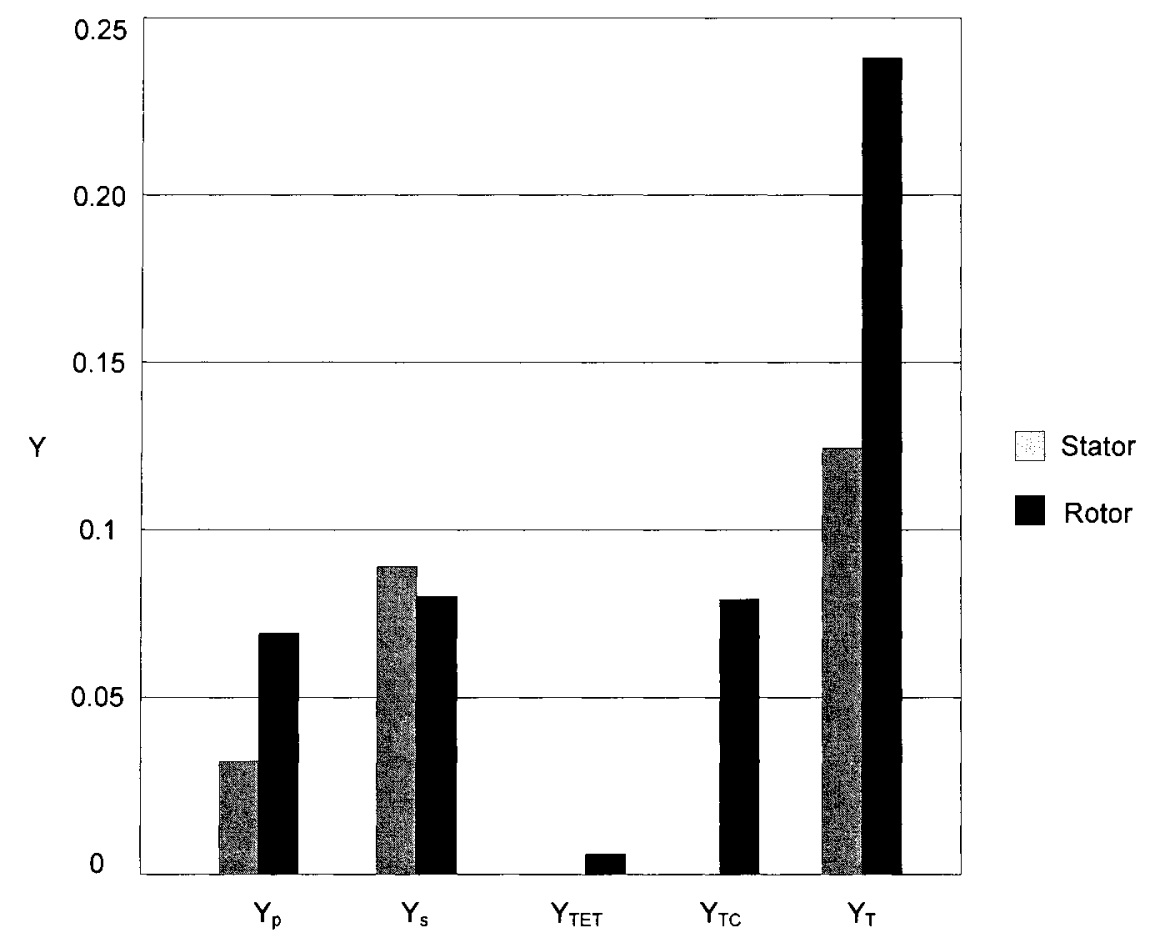

Figure 4.2: Variation in loss mechanisms for gas generator turbine design

\subsubsection{Structural Design and Analysis}

The current section will describe the results calculated in the structural evaluation of the gas generator turbine components. For the rotor calculations, the average hub and tip radii are used. This is a result of the large annular flare that exists as a result of the pressure difference across the blade row. Using these average quantities, a hub-to-tip ratio of 0.781 is calculated. According to Saravanamuttoo et al. (2001), values between 0.714 and 0.833 are regarded as satisfactory. For preliminary design purposes, a good indication of the structural integrity of the turbine rotor can be evaluated by the term $\mathrm{KAN}^{2}$. For the current design, $\mathrm{KAN}^{2}$ is estimated as $3.5 \times 10^{10} \mathrm{in}^{2} \cdot \mathrm{RPM}^{2}$. Typical values indicated by Sjolander (2002) are shown in Table 4.2 . 


\begin{tabular}{|c|c|}
\hline Machine Type & $\mathrm{KAN}^{2}\left[\mathrm{in}^{2} \cdot \mathrm{RPM}^{2}\right]$ \\
\hline \hline High-Pressure Turbine & $4-5 \times 10^{10}$ \\
\hline Shrouded Low-Pressure Turbine & $6-8 \times 10^{10}$ \\
\hline Unshrouded Low-Pressure Turbine & $8-10 \times 10^{10}$ \\
\hline
\end{tabular}

Table 4.2: Typical values for $\mathrm{KAN}^{2}$

By including an estimate for the density of the rotor blades, the program calculates a value for the blade root stress. For the gas generator turbine, the blade root stress is estimated to be approximately $320 \mathrm{MPa}$. Typically, the blade root will experience the maximum tensile stress of the blade and thus will be used in determining the creep life for preliminary design purposes. This life is typically the limiting factor in material selection for turbine components. A discussion of the material selection process will be described in the next section; however, some material properties are used herein as required. In order to achieve the desired component life, set by the design specifications, it was decided to select a specific nickel-base superalloy. The LarsenMiller parameter and fatigue properties (shown later) were obtained from the material's supplier's website in order to predict the creep and fatigue life of the components. Using the maximum blade root stress of $320 \mathrm{MPa}$ and an estimated maximum blade metal temperature of $1055 \mathrm{~K}$, a creep life of 5000 hours is predicted by the Larsen-Miller parameter.

Following the creep life estimation, a fatigue life evaluation was conducted. A gas bending stress of $15 \mathrm{MPa}$ was calculated. As with the blade centrifugal stress, the maximum gas bending stress will occur at the blade root. For fatigue considerations, it is necessary to determine whether the combined loading of the constant centrifugal stress and the intermittent gas bending stress will cause failure. Results for this type of fatigue loading are presented in the form of a Gerber diagram. An example of this diagram is shown in Figure 4.3. Along the horizontal axis lies the mean stress; while, the vertical axis displays the range of the alternating stress. The figure illustrates a number of separate experiments that were conducted for 50 and 500 hour tests; however, for the current design application, the horizontal limits may be extrapolated or interpolated using 
the Larsen-Miller parameter. For this application, it is evident that the gas bending stress is well within the acceptable range based on this diagram.

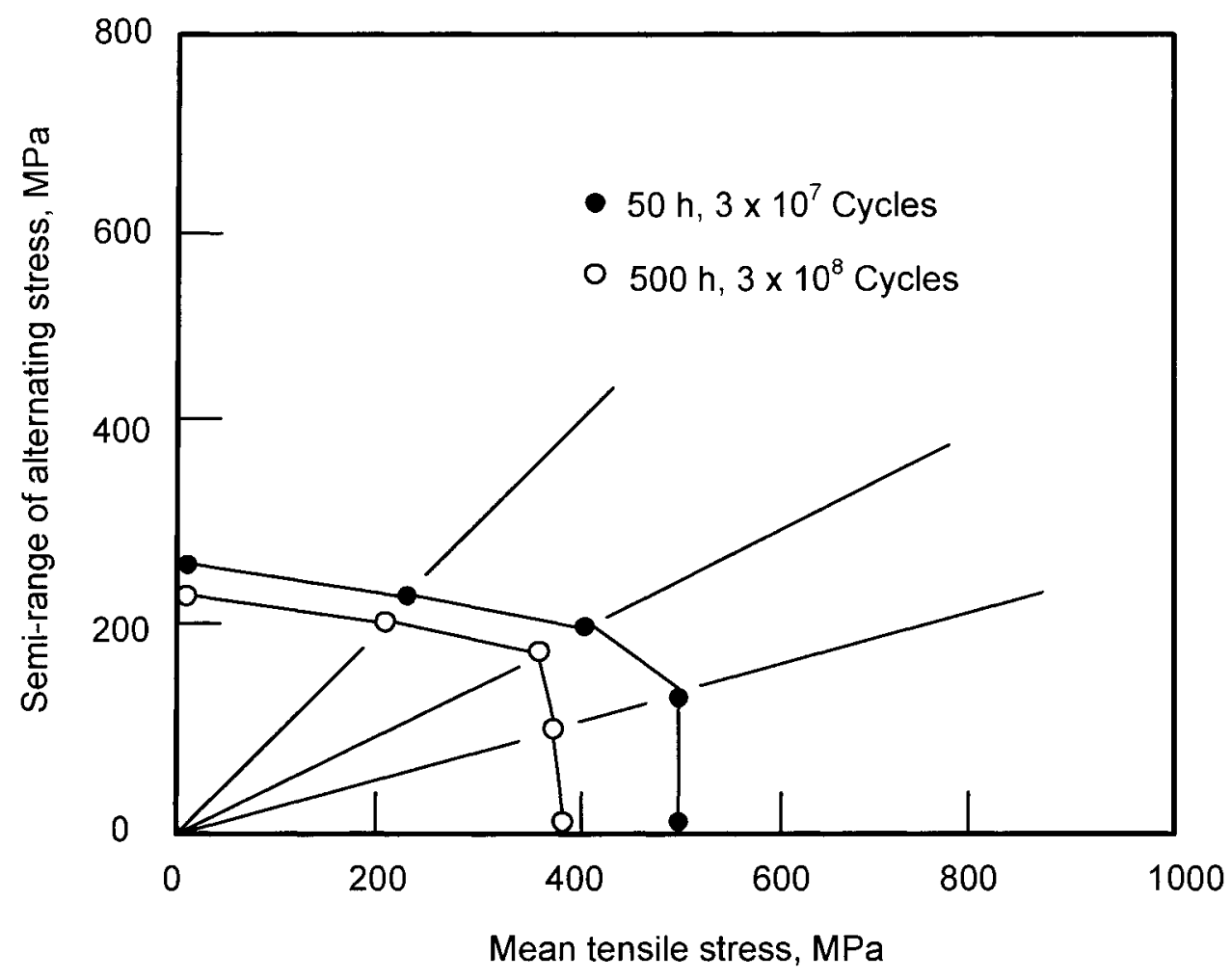

Figure 4.3: Fatigue properties of NIMONIC alloy 95 at $750^{\circ} \mathrm{C}$

In addition to the above discussion, it is valid to note that turbine rotors are designed not to operate in regions of high cycle fatigue (HCF). In the vibration section to follow, it will be seen that any possible method to reduce the effects of HCF will be accounted for. On the other hand, the effects of low cycle fatigue (LCF) are typically more detrimental to gas turbine rotor design. In the case of the current application, LCF is not a major concern due to the life specification of the engine. According to the specifications, the engine must endure 500 start/stop cycles at 4 hours each for a total of 2000 hours. Even with a significant safety factor, a LCF failure occurring within 500 cycles at the prescribed stress levels is unlikely. 
The variation in the radial and tangential disk stresses are presented in Table 4.3. The disk outer radius and thickness are governed by the average hub radius and axial chord component of the blade. The von Mises stresses are also presented for later comparison using the finite element method, described in the following chapters.

\begin{tabular}{|c|c|c|c|}
\hline Radius $[\mathrm{mm}]$ & $\sigma_{\mathrm{r}}[\mathrm{Mpa}]$ & $\sigma_{\mathrm{t}}[\mathrm{Mpa}]$ & $\sigma_{\mathrm{vM}}[\mathrm{Mpa}]$ \\
\hline 0 & 636 & 636 & 636 \\
\hline 21.6 & 615 & 623 & 619 \\
\hline 43.2 & 551 & 586 & 569 \\
\hline 64.8 & 444 & 523 & 488 \\
\hline 86.5 & 295 & 435 & 385 \\
\hline 108.1 & 103 & 322 & 285 \\
\hline
\end{tabular}

Table 4.3: Gas generator turbine disk stress variation

In order to couple the rotor blades and disk, a blade attachment design is presented. Typical blade attachments use the fir-tree design which has been described earlier. A preliminary fir-tree design with three teeth is shown in Figure 4.4. All of the values presented here should be considered approximate and further FEA is required should the design be incorporated in the actual engine.
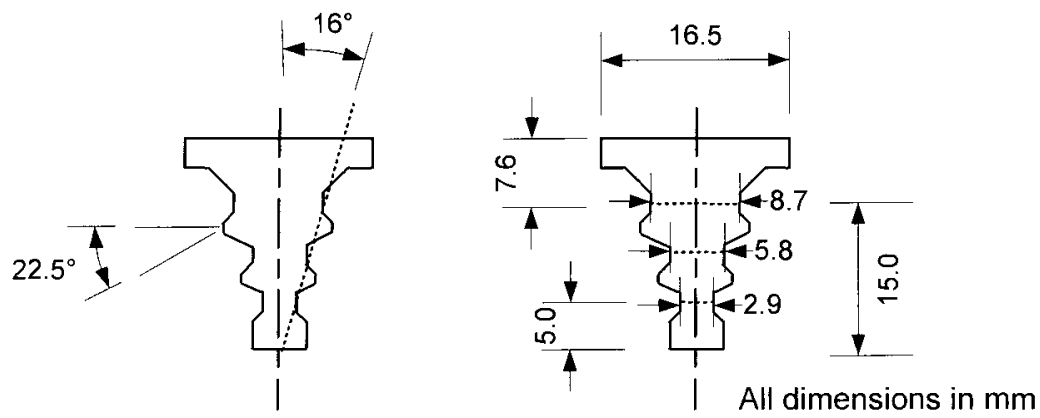

Figure 4.4: Preliminary gas generator turbine blade attachment design

Finally, a burst speed estimation is presented. For preliminary design purposes, the speed of the rotor should be limited to 38,500 RPM. It should be noted that this value is very close to the design speed and has no inherent safety factor. For engine operation, 
a more detailed study is required. This estimate is purposely conservative as it is based on the maximum bore stress and the material yield strength. In practice, a well designed rotor can attain a certain degree of yielding before failure will occur; however, significant finite element analysis should be conducted before attempting to push this limit any further.

\subsubsection{Material Selection}

The material selection for this component is greatly limited by the creep life expectancy of the desired material. A complete discussion on material properties and their requirements for gas turbine design has already been presented in the literature review of Chapter 2. Currently, the selected material is NIMONIC alloy 115, available from the Special Metals Corporation. Fatigue properties were not available for this particular NIMONIC alloy; however, they will be similar to those illustrated in Figure 4.3. This material is a nickel-chromium-cobalt based alloy, strengthened with additions of molybdenum, aluminum and titanium. The material was developed as a creepresisting alloy for service at temperatures up to approximately $1000^{\circ} \mathrm{C}$. Some of the important material properties of this material are listed in Table 4.4.

\begin{tabular}{|c|c|}
\hline \multicolumn{2}{|c|}{ NIMONIC alloy 115} \\
\hline Density $\left[\mathrm{kg} / \mathrm{m}^{3}\right]$ & 8190 \\
\hline Yield Strength [Mpa] & 750 \\
\hline Ultimate Tensile Strength [MPa] & 1000 \\
\hline Young's Modulus [GPa] & 179.5 \\
\hline Poisson's Ratio & 0.317 \\
\hline $\begin{array}{c}\text { Mean Coefficient of Linear Thermal } \\
\text { Expansion [10 }\end{array}$ & 14.4 \\
\hline
\end{tabular}

Table 4.4: NIMONIC alloy 115 material properties at $750^{\circ} \mathrm{C}$

Additional considerations must be made for this material due to the marine nature of the proposed application. For the expected temperature range of the turbine 
components, the active corrosion mechanism is categorized by "low temperature hot corrosion". For this mechanism to be prevented, the material would require a chromium content in excess of 20 percent. Many nickel-based alloys with high creep resistance do not have high chromium levels because the high chromium content is not compatible with high strength. From the material specifications, it is found that the chromium content of NIMONIC alloy 115 is only 14-16 percent. In order to prevent against hot corrosion attack, it would be necessary to apply a CoCrAlY overlay coating with a chromium level in the range of 25-35 percent. By today's standards, applying coatings of this nature is simple and inexpensive and will significantly improve corrosion resistance. Alternatives to the application of coatings may include periodic washing of the entire engine with fresh water to remove any corrosive sulfate deposits, or applying a form of inlet air filtration to reduce levels of salt ingestion.

The topics covered in this section relating to material selection also apply to the design of the power turbine components. For this reason, the design and analysis of the power turbine will be presented next; however, the material selection for this component will not be repeated. It is currently assumed that the power turbine will be fabricated using NIMONIC alloy 115 as well. 


\subsection{Power Turbine Design}

The design specifications and preliminary design decisions of the power turbine components will be presented next. As before, the performance predictions, both aerodynamically and structurally, are also included.

\subsubsection{Design Specifications}

As with the gas generator turbine, the design specifications of the power turbine had been determined at the onset of the project. The power turbine was assumed to be of the axial flow type and to have a total output power of $1 \mathrm{MW}$. The total power was expected to be delivered to a reduction gearbox for use as the customer desired. Using the downstream conditions of the gas generator turbine, it was specified that the power turbine inlet temperature and pressure would be $945 \mathrm{~K}$ and 255,000 Pa, respectively. The expected target efficiency was set the same as before at 88 percent polytropic $(89.4$ percent isentropic).

The rotational speed of the power turbine would only be limited by the manufacturing of the gearbox. For this reason, the customer was consulted on the issue, however, no preferred speed was given. A rotational speed of 27,500 RPM was selected in order to obtain a similar hub radius of the gas generator rotor when combined with the stage loading coefficient. This will limit the amount of radial flow turning in the duct between the two stages.

\subsubsection{Inputs}

Since the required power output of the power turbine is slightly lower than that of the gas generator, a stage loading coefficient of 1.2 was selected. Accompanying the lower stage loading is a lower flow coefficient of 0.5 . In this design, a reaction of 0.5 was selected since the lower stage loading and flow coefficient are a cause of less swirl in the outlet plane than in the gas generator turbine design. 
The inlet flow angle to the power turbine stators is assumed to be approximately 10 degrees. This is a result of the outlet flow angle of the upstream gas generator turbine. In order to obtain a slight increase in performance as well as confidence in the design, a slightly lower Zweifel lift coefficient was used. Although the use of detailed computational fluid dynamics has enabled advances in blade loading through complex modeling of the fluid boundary layer, it was felt that a modest value of 1.0 would ensure the desired performance of the blade row. This is due to the fact that the current project is still in its infancy and CFD is only being used as a validation for this preliminary design tool. It will also be necessary to validate the CFD with experimental testing to obtain confidence in the application of the CFD. This is currently ongoing within the Department of Mechanical and Aerospace Engineering at Carleton University in Ottawa, Ontario. The aspect ratio for the stator and rotor were set to 0.7 and 2.1 , respectively.

\subsubsection{Blade Design}

Once again the program calculates the various flow and metal angles for the hub, meanline and tip radii. Additional calculations are also performed for the fluid states and Mach numbers at each station. Under the given design parameters, a total-to-total isentropic efficiency of 90 percent is predicted and further optimization may be conducted if desired. The meanline velocity triangles $\left(r_{m}=141 \mathrm{~mm}\right)$ are shown in Figure 4.5. In order to obtain the maximum benefit from any outlet swirl of the upstream rotor, it was decided to reverse the rotational direction of the power turbine. Due to the change in rotational direction, the sign convention of Figure 4.5 will also be reversed.

Once again, the axial velocity at Station 2 has been scaled to more accurately depict the flow conditions which will exist in the rotor due to the change in tip radius in the actual engine. For the current design, 15 stator blades and 35 rotor blades are included. 


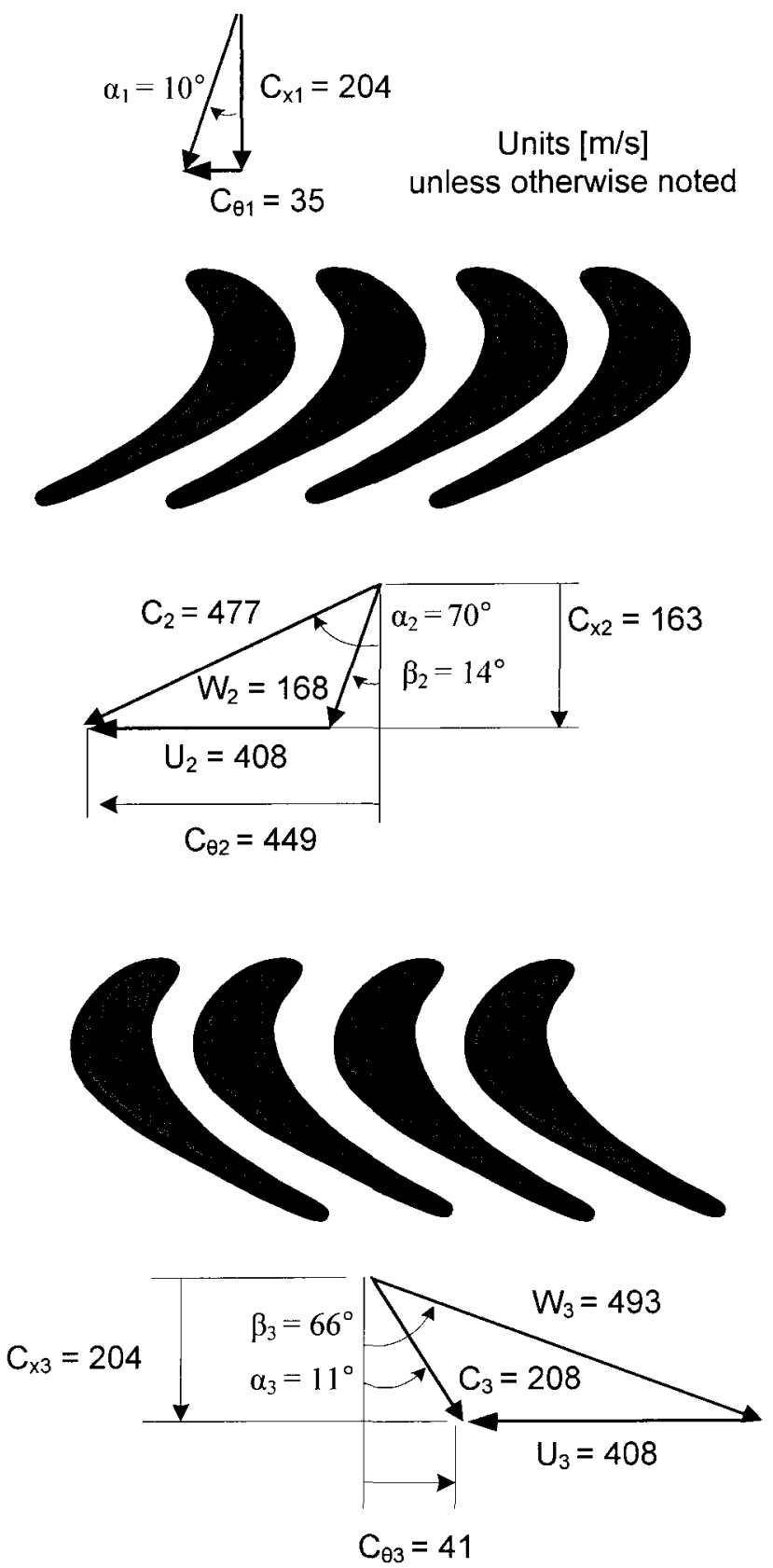

Figure 4.5: Power turbine meanline velocity triangles 
The fluid states and Mach numbers for each station are also included. These results are summarized in Table 4.5.

\begin{tabular}{|c|c|c|c|}
\cline { 2 - 4 } \multicolumn{1}{c|}{} & Station 1 & Station 2 & Station 3 \\
\hline $\mathrm{P}_{0}[\mathrm{~Pa}]$ & 255800 & 245500 & 101600 \\
\hline $\mathrm{T}_{0}[\mathrm{~K}]$ & 945 & 945 & 765 \\
\hline $\mathrm{P}[\mathrm{Pa}]$ & 236700 & 157400 & 92000 \\
\hline
\end{tabular}

\begin{tabular}{|c|c|c|c|}
\hline Hub & \multicolumn{3}{|l|}{} \\
\hline $\mathrm{M}$ & 0.34 & 1.07 & 0.39 \\
\hline $\mathrm{M}_{\text {rel }}$ & & 0.65 & 0.77 \\
\hline
\end{tabular}

\begin{tabular}{|c|c|c|c|}
\hline Meanline & \multicolumn{3}{|c|}{} \\
\hline $\mathrm{M}$ & 0.34 & 0.86 & 0.39 \\
\hline $\mathrm{M}_{\mathrm{rel}}$ & & 0.30 & 0.918 \\
\hline
\end{tabular}

\begin{tabular}{|c|c|c|c|}
\hline Tip & \multicolumn{3}{|l}{} \\
\hline $\mathrm{M}$ & 0.34 & 0.70 & 0.38 \\
\hline $\mathrm{M}_{\text {rel }}$ & & 0.45 & 1.08 \\
\hline
\end{tabular}

Table 4.5: Summary of power turbine fluid states

\subsubsection{Aerodynamic Performance}

A total stage isentropic efficiency of 90 percent has been quoted for the current power turbine design. This efficiency includes a total pressure loss of 4 percent across the stators and a tip clearance of 1 percent. Once again, a large portion of the overall loss exists in the stator vanes presumably due to the low aspect ratio. This is not a primary concern as the target efficiency has been met. A summary of the pressure loss coefficients in both the stator and rotor is shown in Figure 4.6. Note the large difference in the rotor total loss coefficient, $\mathrm{Y}_{\mathrm{T}}$, compared to that shown previously for the gas generator rotor. This difference is largely due to the secondary losses which have been reduced significantly by increasing the aspect ratio of the rotor blade. 


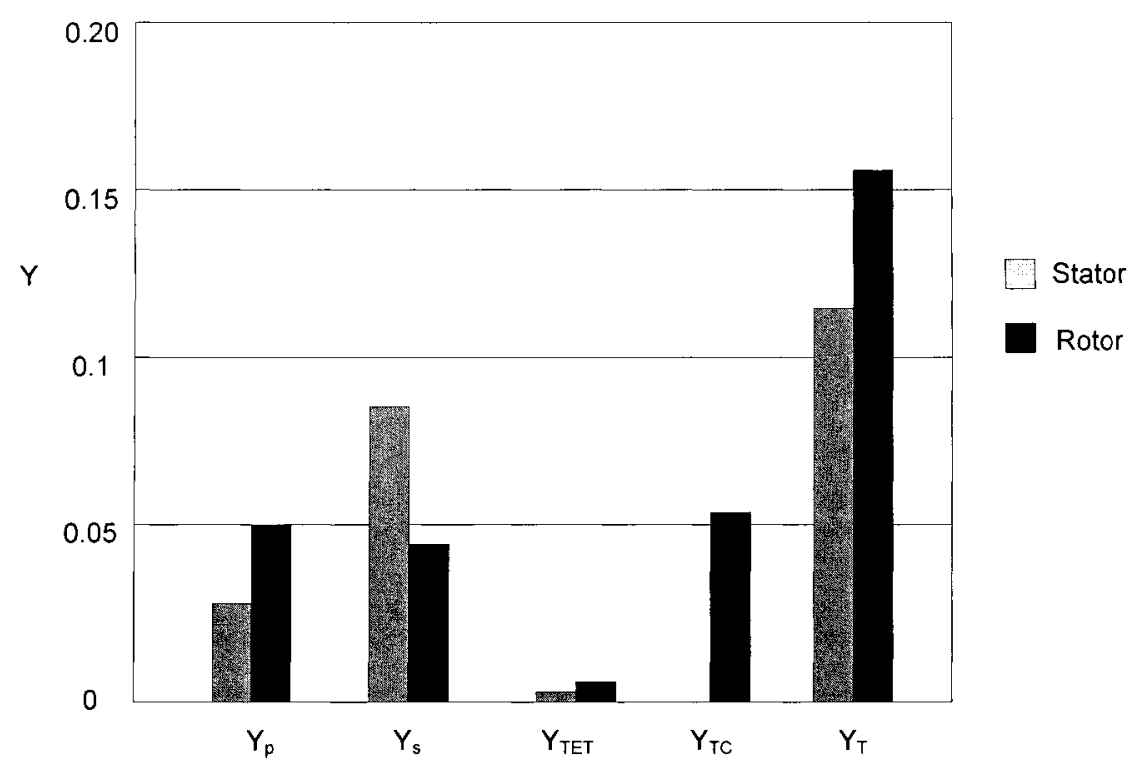

Figure 4.6: Variation in loss mechanisms for power turbine design

\subsubsection{Structural Design and Analysis}

Using the average hub and tip radii, a hub-to-tip ratio of 0.623 was calculated. Although this value is slightly below the recommended range, it could be increased by increasing the axial component of velocity (by increasing the flow coefficient), if desired. The value of $\mathrm{KAN}^{2}$ for this design is approximately $5 \times 10^{10} \mathrm{in}^{2} \cdot \mathrm{RPM}^{2}$. Given that this turbine is an unshrouded low pressure turbine, this value is more than satisfactory, see Table 4.2. Using the same material properties as before, the blade root stress is estimated to be approximately $440 \mathrm{MPa}$. Although this value is significantly larger than the blade root stress for the gas generator turbine, the operating temperature is appreciably less. A maximum blade metal temperature has been estimated at approximately $850 \mathrm{~K}$, thus avoiding any concern for material failure due to creep. Material selection for this component may be revisited if desired.

For fatigue considerations, this component exhibits an even smaller alternating gas bending stress than the gas generator turbine. Furthermore, any concern of HCF will be alleviated by design; therefore, it should not have any effect on the performance of the 
engine. The discussion of LCF presented for the gas generator turbine applies to this instance as well.

The variations in the radial, tangential and von Mises stresses for the rotor disk are presented in Table 4.6. Once again, the outer disk radius and thickness are governed solely by the average hub radius and axial chord component of the blade. These assumptions have been made for preliminary design purposes only. Additional consideration will be required for the detailed design of the rotor disk and additional finite element analysis will need to be performed.

\begin{tabular}{|c|c|c|c|}
\hline Radius $[\mathrm{mm}]$ & $\sigma_{\mathrm{r}}[\mathrm{Mpa}]$ & $\sigma_{\mathrm{t}}[\mathrm{Mpa}]$ & $\sigma_{\mathrm{vM}}[\mathrm{Mpa}]$ \\
\hline 0 & 500 & 500 & 500 \\
\hline 21.8 & 487 & 492 & 489 \\
\hline 43.5 & 446 & 468 & 458 \\
\hline 65.3 & 380 & 429 & 407 \\
\hline 87.0 & 286 & 374 & 339 \\
\hline 108.8 & 166 & 304 & 263 \\
\hline
\end{tabular}

Table 4.6: Power turbine disk stress variation

The preliminary fir-tree blade attachment design for the power turbine is shown in Figure 4.7. Once again, this attachment design is provided for preliminary design purposes only.
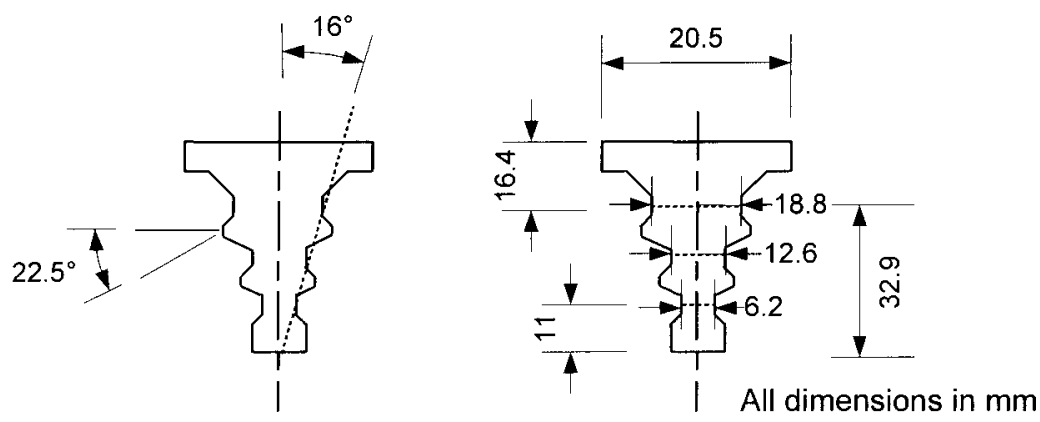

Figure 4.7: Preliminary power turbine blade attachment design 
Further study and analysis is required if a fir-tree blade attachment is to be used in the actual engine. For cost implications, it may be feasible to attempt to cast a solid bladed disk. A solid bladed disk would remove the possibility of additional complex vibration problems attributed with the contact areas of the blade attachments. Additional problems associated with stress concentrations and fatigue failure could also be avoided.

A similar burst speed analysis was conducted for the power turbine rotor. In this case however, a significant margin is attainable due to the difference in design rotational speed. For the power turbine rotor, the conservative estimate for the burst speed is $36,000 \mathrm{RPM}$. Once again, this is the rotational speed at which the rotor begins to yield in the region of maximum stress. Noting that the design rotational speed is of the order of 27,500 RPM, a large margin of safety is automatically achieved.

The following section will describe the design of the turbine casings. Additional material will be provided on the actual design of the turbine components in Chapter 5, where the outputs of the design tool are used to begin generating solid models for fluid dynamic and structural evaluation. 


\subsection{Turbine Casing Design}

In addition to the blade and disk design, the design tool estimates the casing thickness based on the containment of one third of the rotor upon failure (rotor burst). Based on this analysis, the turbine casings for both stages have been designed. The layout and mechanical design are based on the Pratt \& Whitney Canada PW206, an engine with a similar turbomachinery arrangement. The layout indicates the attachment points which are used to fasten the housing to the remainder of the engine. It is assumed that the stator ring will be cast as one piece with the blades attached directly to the stator endwalls. The stator assembly will be fastened directly to the upstream volute or ducting. The casing for the gas generator turbine is a simple annular ring to be overhung from the rear of the assembly. The power turbine stator assembly will also be cast as one piece and fastened at the rear of the assembly, downstream of the power turbine rotor. This component will be attached to the same structure as the gas generator turbine casing. Figure 4.8 shows the proposed turbine housing design.

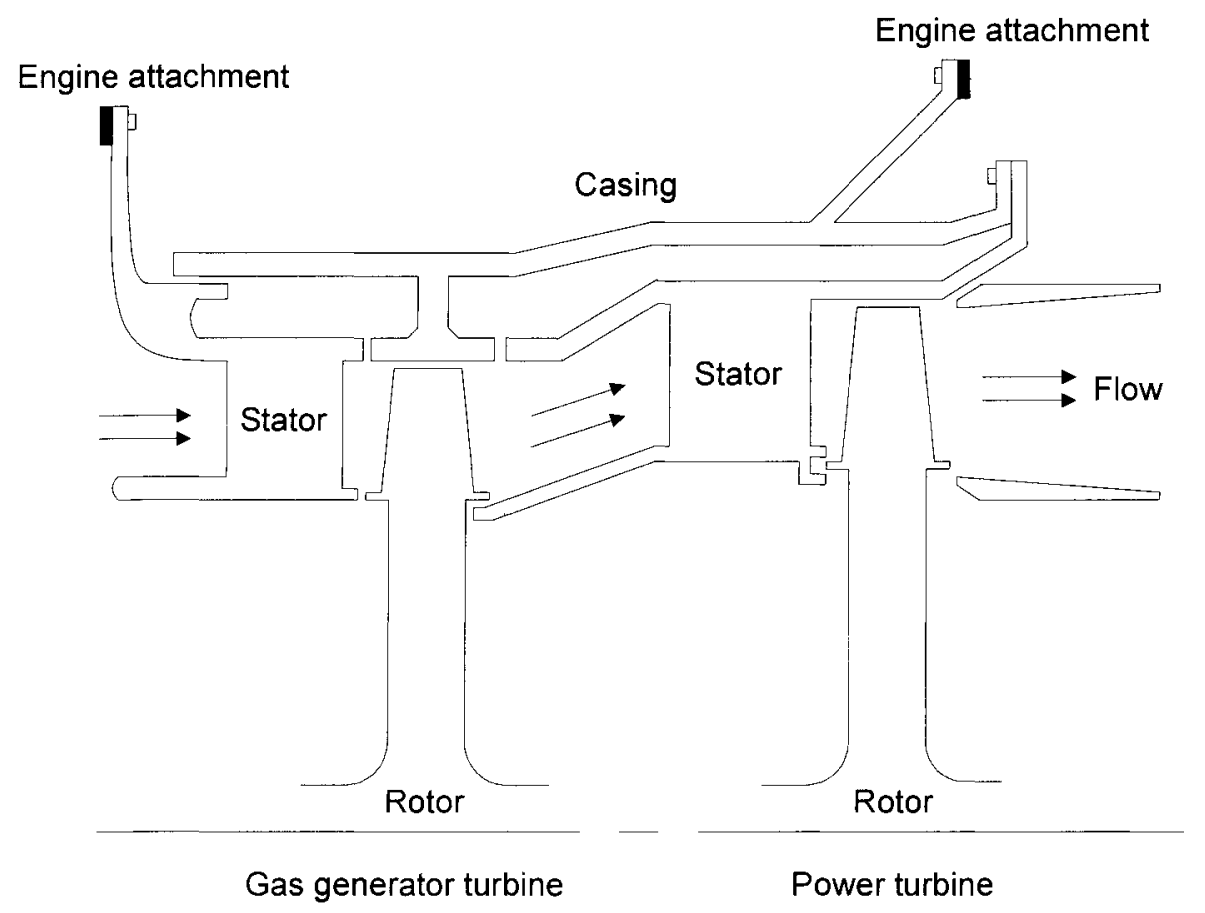

Figure 4.8: Proposed turbomachinery layout and casing design 
Based on the program output, a minimum casing thickness of $10 \mathrm{~mm}$ and $15 \mathrm{~mm}$ would be required for the gas generator and power turbines, respectively. This is the minimum amount of material required to contain one third of the total kinetic energy of the rotor, in the event of a disk failure at the burst speed calculated earlier. It is important that a detailed heat transfer analysis of the turbine casing be conducted as part of the future work. This is required in order to determine the hot running clearance between the rotating blade tip and the stationary turbine casing. It is important to note that the growth rate of the casing will differ from that of the rotor, and a potential for rub exists. This must be avoided at all costs and thus warrants the need for detailed finite element analysis of the turbine rotor and casings. 


\section{Chapter 5}

\section{Methods for Design Tool Validation}

This chapter will describe the methods used to validate the accuracy and usefulness of the design tool described in previous chapters. Since fabrication and experimental testing of the designed components are costly, time consuming, and beyond the scope of this thesis, state-of-the-art CFD and FEA tools were chosen to validate the output of the design tool. Using these available computational tools, the aerodynamic performance and structural evaluation of the components can be verified. Not only do the computational tools validate the accuracy of the design tool, but they also provide a means of estimating how the components will actually behave in operation. By modeling the designed component computationally, full performance characteristics of the component may be generated by simply changing the boundary and operating conditions of the model.

To perform the validation of the design tool, it was chosen to use the software packages CFX-5 and ANSYS 9.0 for the CFD and FEA. Using the output of the design tool, the blade profiles and their radial arrangement were defined using the software package CFX-BladeGen. Using the CFX-BladeGenPlus module, the blades can be screened using a coarse grid and robust turbulence model before being imported into CFX-5 to conduct a complete CFD analysis. Once a satisfactory blade arrangement has been verified aerodynamically, the complete rotor is created in the solid modeling software Pro/ENGINEER Wildfire. From this point, the solid model is imported into the FEA package ANSYS 9.0 to obtain verification of the structural integrity of the components. 


\subsection{CFX-BladeGen}

The CFX-BladeGen and CFX-BladeGenPlus software packages are used to define the blade profiles and their complete three-dimensional shape. The blade geometry may be screened on an aerodynamic basis and modified as needed. This section will provide an outline of the blade generation, meshing, and analysis processes.

\subsubsection{Blade Generation}

In order to begin the validation process, the preliminarily designed blades must be modeled computationally. The blade model is achieved by entering specific results from the design tool into the CFX-BladeGen software. Using the resulting hub and tip radii, axial chord, stagger angle, and blade metal angles, a complete blade can be generated from hub to tip. CFX-BladeGen also requires the user to enter the number of blades specified by the design, so it may partition the annular area into periodic blade passages for analysis. At this point, a complete rotor is defined, and its performance may be evaluated. It should be noted that additional parameters are also required which define the blade curvature and loading distribution. These parameters can have a significant effect on the performance and must be concurrently optimized by trial and error.

\subsubsection{Blade Meshing and Boundary Conditions}

Once the blade geometry has been defined, it is necessary to discretise the flow domain, so the solver can obtain a solution for the given boundary conditions. This is conducted through the CFX-BladeGenPlus module. In this module, a grid density is specified, the fluid properties are entered, and the boundary conditions are applied. It is important to note that CFX-BladeGenPlus has only the Zero-Equation turbulence model available. Finally, the convergence criteria are specified. Two criteria are entered: the maximum number of iterations and a minimum target residual. Once these values have been specified, the solution may begin. The setup for the gas generator turbine rotor is summarized in Table 5.1. 


\begin{tabular}{|c|c|}
\hline \multicolumn{2}{|c|}{ CASE SELECTION } \\
\hline Geometry Unit & $\mathrm{mm}$ \\
\hline Component Type & Rotor \\
\hline Housing Type & Unshrouded \\
\hline \multicolumn{2}{|c|}{ GRID SELECTION } \\
\hline Grid Refinement Factor & 2.0 \\
\hline Grid Inflation Layers & 5 \\
\hline \multicolumn{2}{|c|}{ FLUID PROPERTIES } \\
\hline Fluid Type & Compressible (ideal gas) \\
\hline Specific Heat Ratio & 1.331 \\
\hline Specific Heat $(C p)$ & 1154 \\
\hline Fluid Model & Turbulent \\
\hline \multicolumn{2}{|c|}{ OPERATING CONDITIONS } \\
\hline Inlet Swirl Angle & $71 \mathrm{deg}$ \\
\hline Rotational Speed & 35000 RPM \\
\hline Inlet Total Pressure & $721300 \mathrm{~Pa}$ \\
\hline Inlet Total Temperature & $1200 \mathrm{~K}$ \\
\hline Est. Mass Flow Rate & $5.5 \mathrm{~kg} / \mathrm{s}$ \\
\hline Exit Static Pressure & $223100 \mathrm{~Pa}$ \\
\hline Wall Roughness Height & 0.0 \\
\hline \multicolumn{2}{|c|}{ SOLUTION } \\
\hline Maximum Iterations & 1000 \\
\hline Target Residual & $1 \times 10^{-6}$ \\
\hline
\end{tabular}

Table 5.1: CFX-BladeGenPlus analysis requirements

Once the convergence criteria have been met, the solver will terminate, and the user may begin post-processing the results. Prior to post-processing the results from any computational software, it is necessary to ensure that convergence was actually obtained (such that the solver did not terminate as a result of reaching the maximum number of iterations). For this reason, the maximum number of iterations was generally set to a 
very high number (about $10^{6}$ ) and the residuals were monitored manually. The solution was then allowed to continue until the residuals asymptotically reached their lowest value.

\subsubsection{Post-processing Results}

Once the solution has converged and properly terminated, the results of the solver may be viewed. In CFX-BladeGenPlus, this is a relatively easy process; deciphering the results, on the other hand, is complicated. A number of pre-selected tables, figures, and charts are available under the results tab in the BladeGenPlus menu. These figures are generated automatically and summarized in an HTML document. The figures contain plots of blade to blade pressure distributions, vector plots, loading diagrams, etc. Additional plots may be generated by creating new report objects, or existing plots may be edited and modified. Included in the HTML report are tables summarizing the resulting inlet and exit flow velocities, flow angles, pressures, and temperatures. Also included is a performance summary calculated for specified machine type. 


\subsection{CFX-5}

Once the blade geometry has been modeled and analyzed by CFX-BladeGen and CFX-BladeGenPlus, a more comprehensive CFD analysis is performed using CFX-5. The use of CFX-5 will allow additional control of the turbulence models, grid density, solution accuracy, time step control, tip clearances, stator-rotor interaction, and much more. Using CFX-5, an example of a complete stator-rotor simulation with tip clearances will be presented. The results and discussions regarding the actual designs with various turbulence models and grid densities will be presented in the following chapter.

Similar to any state-of-the-art computational software package, there exist numerous options and available modeling methods for the user to select. For this reason, the learning curve of the software is quite steep, and it is recommended that any new user conduct a number of the self-learning tutorials before conducting any of the analyses presented herein. This section will provide a methodology for conducting a comprehensive aerodynamic analysis to validate the design tool presented earlier. The methodology may also be used for determining the performance of other axial turbine designs as required.

\subsubsection{Meshing}

This section will provide the reader with two separate methods for generating a mesh compatible with CFX-5. The first method uses the mesh generator available in CFX-BladeGenPlus. In order to generate the mesh, the user must complete the case and grid selection sections of Table 5.1. Once the grid has been generated, the user must save the file as a definition or results file. This file is now available for use in CFX-5. These meshes are relatively coarse, and there is little control of the meshing parameters.

A finer, more controlled mesh may be generated using ANSYS Workbench. This method requires the BladeGen geometry file to be first imported into the ANSYS Workbench tool. The creation of the blade geometry with tip clearances for use in CFX- 
5 in presented in Appendix D1. Even greater mesh control is available with the use of additional software packages such as ANSYS ICEM-CFD or ANSYS TurboGrid. The latest version of ANSYS Workbench 10 has incorporated ANSYS TurboGrid into its meshing software. All of the methods presented herein were conducted using ANSYS Workbench version 9.0 and CFX-5 version 7.1 .

Various forms of mesh spacing are available in CFX-Mesh. As the detailing and description of each of these are not the focus of the thesis, it will be up to the reader to investigate these further, if desired. An example of the complete mesh generation for one of the gas generator turbine rotor passages is presented in Appendix D2.

\subsubsection{CFX-Pre}

CFX-Pre is the pre-processing module of CFX-5. Its role is to set up the simulation by specifying the geometry, turbulence models, boundary conditions, fluid properties, etc. For turbomachinery simulations, it is recommended to use the Turbo option in CFX-5. This will aid the user in the specification of the required mesh, fluid properties, and boundary conditions relevant to turbomachinery application. CFX-Pre permits the definition of multiple domains such that stationary and rotating reference frames may be used to model stator and rotor interaction. Once the geometry and mesh have been defined, the various interfaces and periodic boundaries are specified. Multiple options exist for simulating the change in reference frame across these interfaces which will be described later. Once the model has been fully specified, Turbo Mode is closed, and the General Mode of CFX-5 is activated. Here, additional solver and output control parameters may be edited and the solution can be started. An example of the complete setup used to model the gas generator turbine stage is given in Appendix D3.

\subsubsection{CFX-Solver}

The CFX-Solver Manager is typically started with the definition file created in CFX-Pre. All of data required for the simulation is contained within this file. At this 
point, the user has the option of specifying an initial values file. The initial values file is most often a completed solution for a similar case. It may be used to start a new simulation on the same mesh, or it may be interpolated onto a new mesh defined by the definition file. It is typically used to reduce the number of iterations for a given solution, or to improve the convergence of a poorly solved solution.

At this point, the solver begins the solution of the equations governing the flow. In order to judge the convergence of the solution, the resulting residuals are tabulated and plotted in the Solver Manager window. Once the residuals have converged to the desired limit, the solution is finished. As with the BladeGenPlus solver, it has become common practice to allow the residuals to converge asymptotically to ensure absolute convergence, and then verify that their absolute values are below the prescribed limit. For all solutions presented herein, the convergence criteria ensured that the maximum residual was below $10^{-4}$. This will conclude the current discussion on the CFX-5 Solver. Additional resources on the solver parameters are available in the CFX help manual.

\subsubsection{CFX-Post}

The CFX-Post module is used for post-processing the results of the simulation. Upon completion of the simulation, the CFX-Solver writes a results file (*.res) for use by CFX-Post. Using this module, a variety of different plots, charts, and contours may be generated. As with the meshing, pre-processing, and solving capability, the postprocessor has many additional features compared to the BladeGenPlus software described earlier. Complete three-dimensional views and animations can be generated. Additional calculations and user defined functions can also be used.

Similar to the pre-processing module, an additional Turbo Mode option is provided in CFX-Post. This option is convenient for conducting post-processing calculations specific to turbomachinery applications. It allows the simple application of blade-to-blade plots, loading diagrams, efficiency calculations, as well as velocity component calculations in both frames of reference. It further permits the generation of a 
gas turbine performance report for determining the overall performance of the blade row or stage. This report will include inlet and outlet temperatures and pressures, specific work output, and the overall stage efficiency. 


\subsection{Pro/ENGINEER Wildfire}

In addition to the validation of the aerodynamic performance estimated by the design tool, it was also necessary to validate the accuracy of the structural evaluation. This validation was conducted using the finite element analysis software ANSYS 9.0 to examine the various stresses and temperature distributions within the rotor. In order to perform the finite element analysis on the designed components, solid models of the components must first be created. The creation of these solid models was conducted using Pro/ENGINEER Wildfire.

The blade designs were imported into Pro/ENGINEER Wildfire using the boundary curves defined by CFX-BladeGen. Surfaces were then created from the boundary curves and merged together. A generic shaped rotor disk, with dimensions equal to the output of the design tool, was then created. The blade surfaces were then patterned around the rotor disk to match blade number output by the design tool. The blade surfaces and rotor disk are then merged together and solidified to create the solid model. Appendix D4 contains a step-by-step procedure for carrying out these actions. 


\subsection{ANSYS 9.0}

The software package ANSYS 9.0 was used to validate the structural integrity evaluation performed by the design tool. Using the design tool, preliminary estimates of disk and blade stresses were conducted. These stresses were then used to estimate the creep and fatigue life of the turbine rotor. The stress estimates performed by the design tool take into account various simplifying assumptions and it was deemed necessary to investigate the validity of these assumptions. The use of ANSYS 9.0 will provide additional results regarding the temperature distribution and vibration characteristics of the turbine model. To date, these considerations have not been incorporated in the design tool.

The methodology used to perform the structural evaluation of the turbine rotor in ANSYS 9.0 is detailed in this section. The results of the inertial loading are used to validate the calculations performed within the design tool. Additionally, the methodology used to produce the thermal and vibration analyses will be presented.

\subsubsection{Inertial Analysis}

This section will describe the inertial loading of the turbine rotor conducted in ANSYS 9.0. An inertial loading simulation is analogous to spinning the rotor at a desired rotational speed. The results of this analysis will be presented in Chapter 6 .

Using the solid models generated in Pro/ENGINEER Wildfire, the geometry of the gas generator and power turbines are first imported into ANSYS 9.0. The material properties are specified and the mesh is generated. For non-complex shapes with relatively little curvature, the automatic mesh generator using the Smart Size option is recommended. However, for highly complex shapes with a large extent of curvature, such as a gas turbine blade, it is recommended to use more advanced meshing techniques. It was found that the most efficient way to mesh the current blade models was to first divide the overall volume into smaller individual volumes. Then, localized size controls 
were specified for various areas and the volumes were meshed separately. If this process is conducted properly, it is possible to generate a variety of unstructured and structured grids. In order to further reduce the required computational resources, a fractional model is used. To account for the fractional model, a cyclic sector is defined.

The initial setup of each of the ANSYS 9.0 simulations is virtually the same. For some cases, different material properties are required, and for others a different solid model may be used; however, the setup to this point is identical. After the mesh has been generated, different loadings are applied for each application. For the inertial analysis, a structural displacement constraint is applied to the end of the rotor shaft. This will constrain any elements at this point in the shaft from moving in any direction. It is assumed that the constraint will occur in the same vicinity as the bearing. Following this, a structural inertial load is applied to the model. This will subject the entire gas generator turbine rotor to a constant angular velocity of 35,000 RPM.

ANSYS solutions of this nature may take anywhere from a few seconds to an hour depending on the number of elements in the solution. This is significantly shorter than the CFX solutions described earlier, which can take up to a few days to complete. Once the solver has completed, the cyclic model may be expanded, and the stress distributions can be analyzed.

\subsubsection{Thermal Analysis}

Thermal analysis simulations were also conducted to evaluate the turbine blade and rotor design. Although these thermal results are not directly required for the validation of the design tool, they do play a strong role in determining the structural integrity and safe life of the components. The results of these simulations also play a key role in determining the thermal expansion and thus the amount of allowable clearance between various components, especially between the blade tip and casing. 
For the thermal analysis, the same setup procedure and mesh sizing of the inertial analysis may be used. Although this method requires the user to delete all of the load data from the previous analysis, it was found to be much more efficient than attempting to achieve another suitable mesh. Additional material properties are also required for the thermal analysis.

To perform the thermal stress analysis, a temperature distribution must be specified. For preliminary design purposes, the maximum and minimum blade temperatures are assumed to be on the order of magnitude of the static temperatures calculated in the design tool. Using a temperature boundary constraint, the inlet static temperature was applied to the leading edge. Similarly, the outlet static temperature was applied to blade trailing edge. ANSYS 9.0 automatically assumes that all of the other surfaces are fully insulated. At this point, the temperature distribution within the model may be solved, and a thermal results file will be created. Once this is complete, an additional analysis is required to produce an estimate of the thermal stresses within the model.

This analysis provides an indication of the thermal stress distribution within the blade and rotor disk. It is far from complete, as convection or radiation heat transfer has not been applied. This will be one of the most important tasks of future designers.

\subsubsection{Vibration Analysis}

A preliminary vibration analysis has also been conducted to ensure the structural integrity of the turbine components. To avoid complex situations dealing with coupled blade and disk vibration scenarios, and the effects of cyclic constraints in vibration problems, a vibration analysis on a single blade was performed.

The blade was created from the Pro/ENGINEER solid model. A single volume was constructed comprising of six separate areas. These areas include the hub and tip surfaces, suction and pressure surfaces, as well as the leading and trailing edges. Any 
rounds and fillets used in the structural design were omitted, as they contribute little to the stiffening of the blade section. By creating the volume from such simple shapes, it was possible to create a swept structured grid with relatively fewer elements.

In this case, a modal analysis is selected. It is necessary for the user to specify the number of modes to extract from the analysis options. ANSYS 9.0 also has the capability of conducting a modal analysis which will calculate the mode shapes of a pre-stressed structure. This effectively increases the natural frequencies for each of the modes due to the effect of centrifugal strengthening. For this analysis, a static inertial loading (described in Section 5.4.1) is first conducted followed by the modal analysis just described.

The methodologies presented in this chapter provide the reader with a thorough understanding of the validation process used for the design. Furthermore, the methods offer future users the option to conduct their own simulations, for similar turbine components using CFX-5 and ANSYS 9.0. Appendix D is provided to explain these methodologies in greater detail. The following chapter will detail the results obtained from CFX-5 and ANSYS 9.0 in an attempt to validate the turbine design tool. 


\section{Chapter 6}

\section{Results and Discussion - CFD \& FEA}

The complete preliminary design of two gas turbine stages has been presented. Along with the design, an estimation of the aerodynamic performance and structural integrity of the components has been conducted by the design tool. The scope of this chapter is to compare the results from CFD and FEA to that obtained from the preliminary design tool. The purpose of this procedure is to validate the results of the preliminary design tool as well as to gain confidence in the ability of the components to meet the desired performance specifications.

Due to the sophistication and complexity of the commercial CFD software, CFX5, numerous options exist for turbulence modeling, solver accuracy (advection schemes), grid refinement, modeling techniques, etc. The first section of this chapter will describe a number of options that were investigated throughout gas turbine design stages. Based on these results, a distinct set of options was chosen to perform the validation of the design tool. The results of these CFX-5 simulations are then compared to the outputs of the design tool which have been presented earlier.

Next, the structural evaluation is performed in ANSYS 9.0. Using the methodologies explained previously, inertial, thermal, and vibration analyses were conducted, and the results are presented. These results are then compared with the results which were obtained from the preliminary design tool. 


\subsection{Aerodynamic Validation}

This section describes the settings and options that were explored throughout the use of CFX-5 for the validation of the design tool. Following this, a grid independence study is presented. Next, the aerodynamic performance of the gas generator turbine and power turbine using CFX-5 is explored. These results are compared with the results output from the design tool.

\subsubsection{Settings}

A number of CFX-5 settings were tested and evaluated throughout the development of the two turbine stages. Many of the settings had an insignificant effect on the overall performance of the turbine components; however, it is the combined effect of all the correct settings that is important in determining an accurate prediction of the turbine performance. Variations in the turbulence and heat transfer modeling, tip clearance, and advection schemes resulted in the largest differences. A transient simulation of the stator-rotor combination was attempted, as well as different interface connections between the stationary and rotating domains; however, these options seemed to have little effect on the overall stage performance. 
Throughout the course of the turbine development, a number of simulations were conducted which included various component combinations. The various component combinations included:

- a single rotor blade passage

- a single rotor blade passage with tip clearances

- a single stator blade passage

- a combined stator-rotor blade passage *

- a combined stator-rotor blade passage with tip clearance

(*) a single simulation was conducted with a combined single-stator-blade/dual-rotorblade passage in order to investigate the effects of the area based pitch ratio defined by CFX-5. For this application, the area based pitch ratio is defined as the ratio between the stator outlet area and the rotor inlet area. According to the CFX manual, "the computational accuracy degrades rapidly with increasing pitch ratio" (Anonymous, 2004). This is a result of the circumferential variation of the flow quantities that are not transmitted across the interface using the Stage option for the domain interface. By modeling two rotor blade passages, and one stator blade passage, the pitch ratio is reduced from 2.16 to 1.08 . In the cases analyzed, this had a negligible effect on the performance of the stage. 
The single rotor blade passage simulations were conducted early in the development of the gas generator turbine rotor. The simulations were conducted mainly as part of a screening process of the early design decisions of the turbomachinery development. Once these simulations were clearly understood, the addition of tip clearances was simulated. Using the methodology outlined in Chapter 5, a two percent tip clearance was added to the gas generator turbine blade passage. Unfortunately, due to the relative sizes of the blade passage and blade tip area, a one percent tip gap could not be successfully generated. This was not the case for the power turbine rotor, where a one percent tip gap was successfully generated and meshed. With the effects of tip clearances, an immediate decrease of approximately three percent in the gas generator turbine efficiency and power output was realized. A slightly lower loss of approximately two percent was realized in the power turbine efficiency and power output. These results are consistent with literature (see Figure 2.12) due to the smaller tip gap experienced on the power turbine.

At this point, it was realized that the magnitude of the efficiencies was still rather unrealistic. When this was investigated, it was realized that the effects of viscous work were not being accounted for. By incorporating these effects in the CFD model, the turbine efficiency dropped an additional two percentage points. Viscous work refers to the work done on the fluid by viscous forces, and it is defined as a scalar product of shear stress and velocity. By including the additional viscous work term in the heat transfer model, a more accurate performance estimation was obtained. This is a result of the high degree of flow turning and secondary flows that are experienced in the turbine rotor. The large swirl components and rotating flows incur large shear stresses which in turn create the viscous work and the loss in performance. The total energy heat transfer model was selected over the isothermal or thermal energy models since it includes the effects of kinetic energy. The CFX documentation recommends the use of this model for gas flows where the Mach number exceeds 0.2.

The next step was to analyze the aerodynamic performance of the stator blade rows. For the first iteration, the design program outputs are used in conjunction with the 
default CFX-BladeGen settings. This results in a relatively poor stator performance. In order to obtain a stator with acceptable pressure loss estimations, a significant amount of iteration must be performed on the blade profiles in CFX-BladeGen. This is required to obtain a desirable blade loading. Future considerations should be made for obtaining an additional tool capable of creating and analyzing two-dimensional blade profiles. To obtain acceptable pressure losses, a relatively thin blade trailing edge was selected. This had the effect of reducing the pressure losses occurring due to the stator wake. It was also found that a higher than desired pressure loss occurred as a result of the large downstream length of the flow passage. This length is required to obtain proper flow stabilization and convergence; however, it leads to a large pressure loss due to the strong cross flow velocity component required for the rotor inlet. This is avoided by conducting a combined stator-rotor simulation.

Numerous combined stator-rotor simulations were conducted throughout the development of the turbine components. In these cases, two separate domains are modeled, as described in the methodology. To attach the domains, a domain interface is required. Due to the change in reference frame, three options exist in CFX. These include: Frozen Rotor, Stage, and Transient Stator-Rotor. In the cases analyzed, little difference was found in the overall stage performance for the different models. For the final analyses, it was chosen to select the Stage option, as it was felt that this option was the best trade-off between accuracy and computational effort. According to the CFX manual, the Frozen Rotor option is the least demanding computationally, but the losses that would be incurred as the flow is mixed between the stationary and rotational reference frames are not modeled. The Transient Stator-Rotor option, on the other hand, correctly predicts the true transient relative motion between a stator and rotor passage. This option ultimately accounts for all interaction effects between components that are in relative motion to each other. The main disadvantage of this method is that it is computationally expensive in terms of simulation time and disk space. It was felt that this degree of accuracy was not required for the initial validation of the design tool. Using the Stage option, both blade passages are solved simultaneously with circumferential averaging of the flow quantities between the rotating and stationary 
domains. The averaging at the frame change interface incurs a one-time mixing loss. Although this mixing loss is large enough to "mix out" any velocity profile across the interface, it provides the basic necessities required to validate the design tool.

The last two options to be discussed include the selection of turbulence models and advection schemes. A brief introduction on turbulence modeling has been given in the literature review. For most of the simulations previously discussed, two separate turbulence models were examined. The k-epsilon model was first examined. This model is stable and numerically robust. For comparison purposes, the SST model was also investigated. According to the literature, the epsilon equation typically predicts the onset of separation too late. For this reason the k-omega based SST model was selected. This model is recommended for high accuracy boundary layer simulations. In a general sense, the SST model improved the stage performance by one half to one whole percent. As expected, the k-epsilon model proved to converge more easily than the SST model.

The complete benefits of the SST model were not fully realized. It is recommended that the computational mesh include at least 10 grid points within the boundary layer for the SST model. This would contribute to a mesh density much too fine for the desired application. For the SST simulations conducted, the value of $\mathrm{y}^{+}$ ranged from about 5-25. The term $\mathrm{y}^{+}$is defined as the dimensionless distance between the first and second grid points off the wall. It is used for determining the type of flow model to be used for modeling the flow near a wall. According to the CFX documentation, the use of scalable wall functions requires a $\mathrm{y}^{+}$value in the range of 20 100. On the other hand, the use of a low Re number method for near wall modeling (the main advantage of the SST model) requires a $y^{+}$value less than about two. All of this considered, CFX uses automatic near-wall treatment for k-omega based models. This method will use scalable wall functions in the cases where the boundary layer is not fully resolved, and automatically switch to the low Re number method as the mesh is refined.

For the selection of advection schemes, a second order high resolution scheme is generally recommended. Two additional schemes are available, upwind and specified 
blend factor. The upwind scheme is entirely first order and is numerically stable. Although this method is very robust, it is also susceptible to numerical diffusion or 'gradient smearing'. Using the specified blend factor, a value is chosen between 0 and 1 , reducing the diffusive properties of the upwind differencing scheme. If a value of 1 is chosen, the solution is essentially second order accurate. A value of 0.75 will add 75 percent of the second order correction to the upwind difference solution. The high resolution advection scheme computes the blend factor locally to be as close to 1 as possible. This has the effect of reducing the order locally where the flow has little variation or discontinuities yet maintaining the desired second order accuracy overall. It was found that some solutions would not fully converge at the high resolution setting. This was generally found on the higher node count simulations. In these cases, a specified blend factor of 0.75 was required in order to obtain convergence based on the desired criteria. The 0.75 blend factor had a significant effect on the stage performance. In some cases as much as 1.5 percentage points were lost on the total-to-total isentropic efficiency.

Based on the above discussion, a summary of the recommended settings for the design tool validation are presented in Table 6.1. 


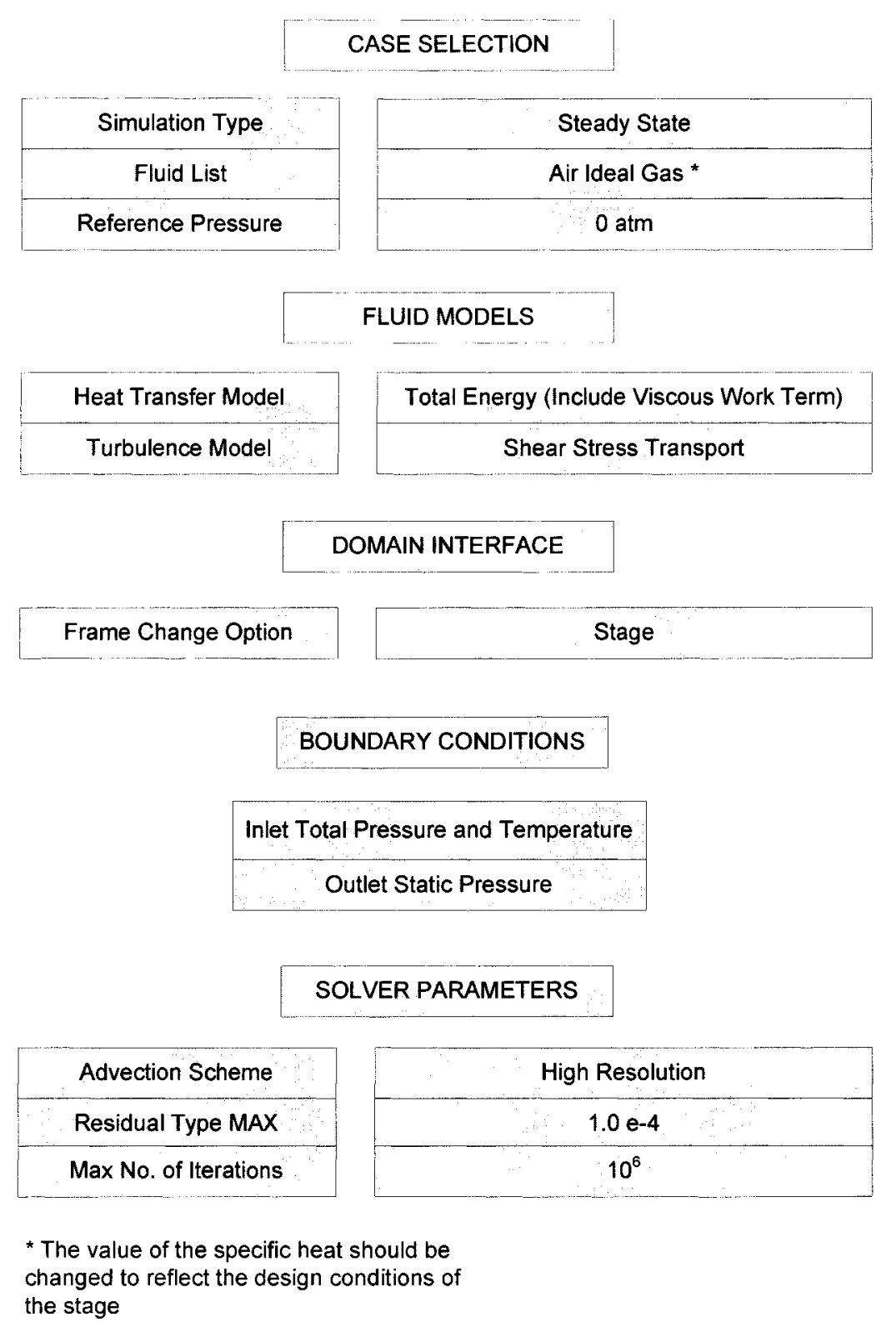

Table 6.1: Summary of simulation settings used in CFX-5

\subsubsection{Grid Sensitivity}

To ensure the validation process is independent of the computational mesh, a grid sensitivity study was conducted. This study is performed to ensure an adequate number of elements and nodes are used in the simulations. Ideally, the results will converge to the exact solution as the grid is refined, and it is up to the designer to make the trade-off between solution accuracy and computational effort. A range of grid densities was used 
in the solutions of the various component combinations described previously. It was found that the grid density did not have as much of an effect on the overall stage performance as what one would have earlier thought. This may be due to the fact that a minimum number of nodes are required to obtain an adequate convergence of the solution.

To examine the effects of grid density, three sets of results are presented. The number of nodes quoted for each set of results includes the sum of the two blade passages (stator + rotor). The solutions are completed converged (the maximum residual is less than $10^{-4}$ ) and include the effects of a two percent rotor tip clearance. The results are summarized in Table 6.2.

\begin{tabular}{|c|c|c|c|}
\hline Simulation & Nodes & $\eta$ & Specific Work $[\mathrm{kJ} / \mathrm{kg}]$ \\
\hline 1 & 110,000 & 88.0 & -289.5 \\
\hline 2 & 220,000 & 88.2 & -289.8 \\
\hline 3 & 490,000 & 88.1 & -289.6 \\
\hline
\end{tabular}

Table 6.2: Grid sensitivity study

As one can clearly see, the node count has little influence on the overall stage performance of the gas generator turbine. The three grids used in the sensitivity study are shown in Figures 6.1, 6.2, and 6.3. For each grid density, three planar views are used to show the various grid densities. The first view (i) illustrates two stator and four rotor blade passages at 50 percent span (meanline). The second view (ii) illustrates the use of inflation layers on the blade surface as well as the grid density adjacent to the rotor blades. Finally, the third view (iii) illustrates the grid density within the tip gap as well as the use of inflation layers on the hub and casing (Figures 6.2 and 6.3 only). 
(i)

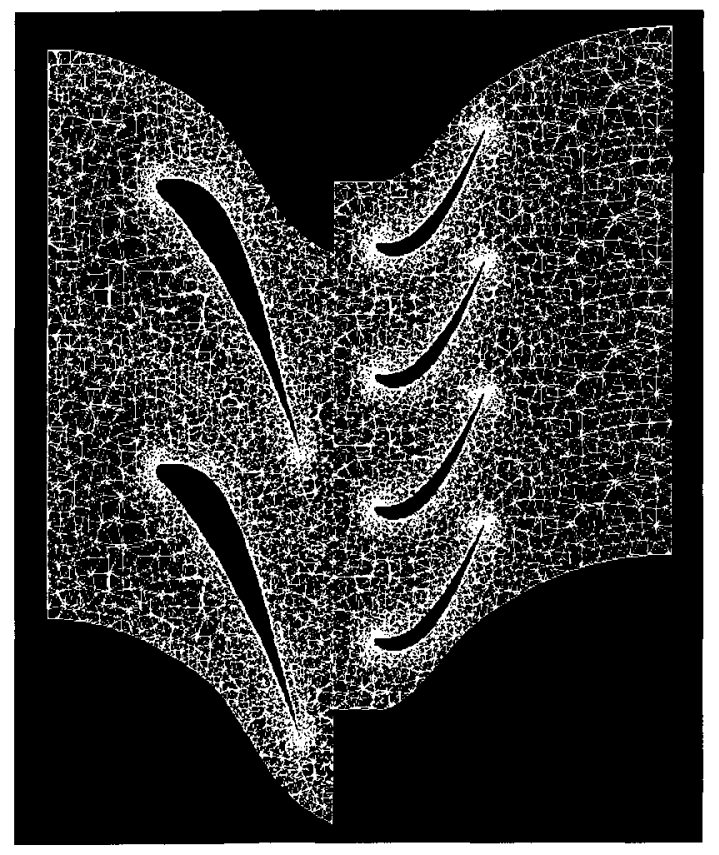

(ii)

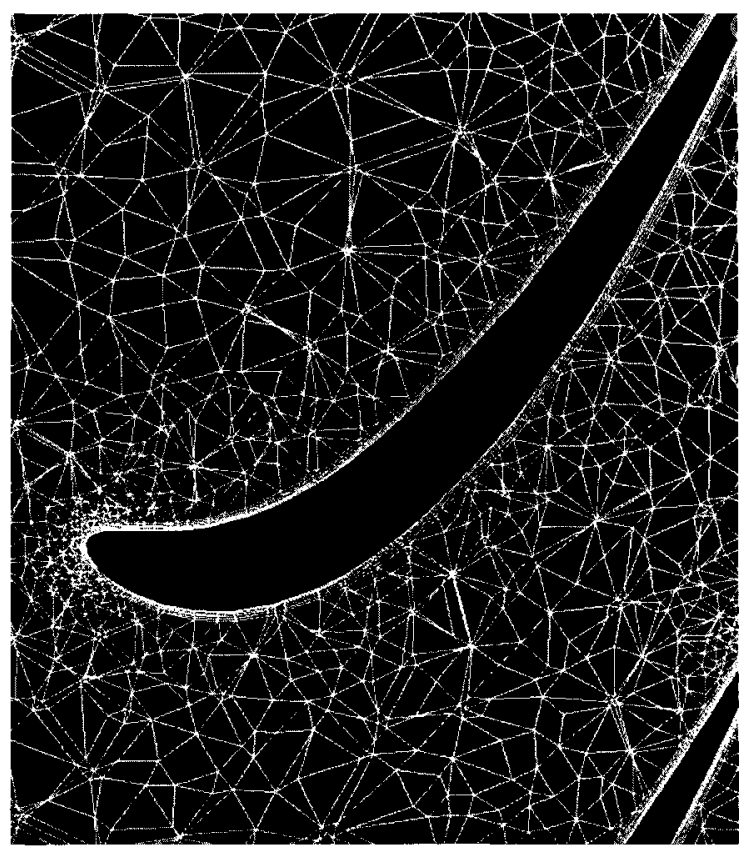

(iii)

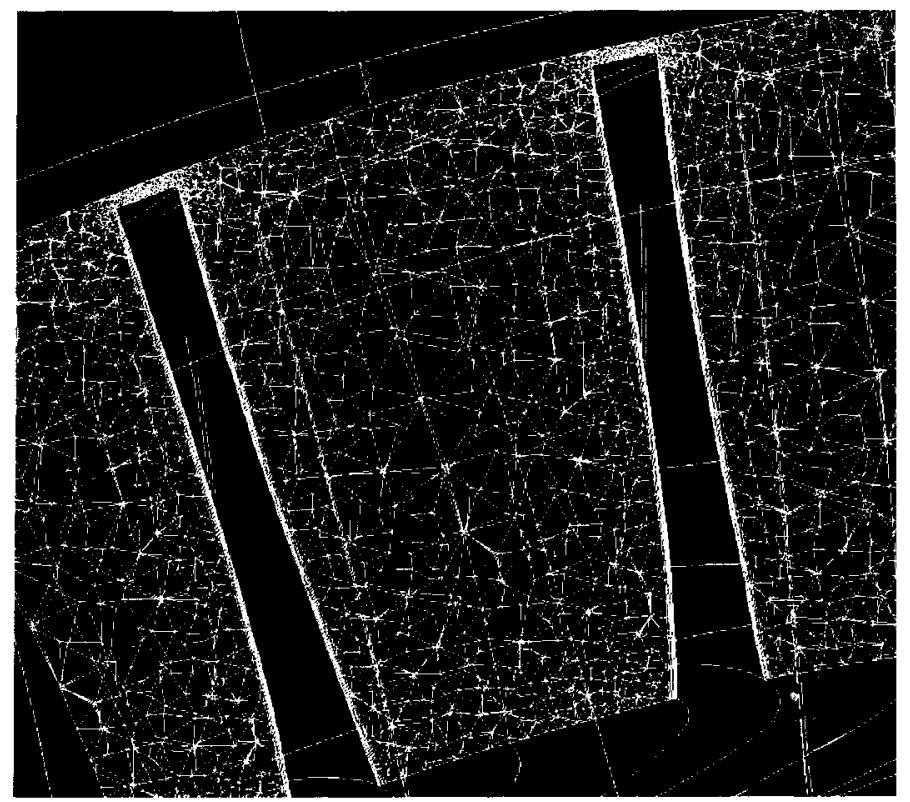

Figure 6.1: Grid density for 110,000 nodes 
(i)

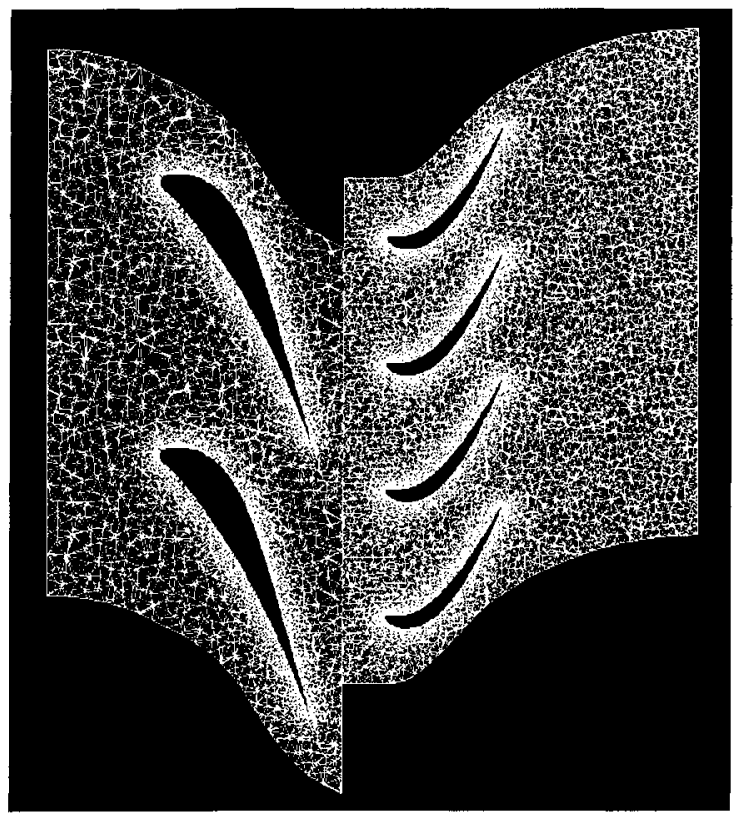

(ii)

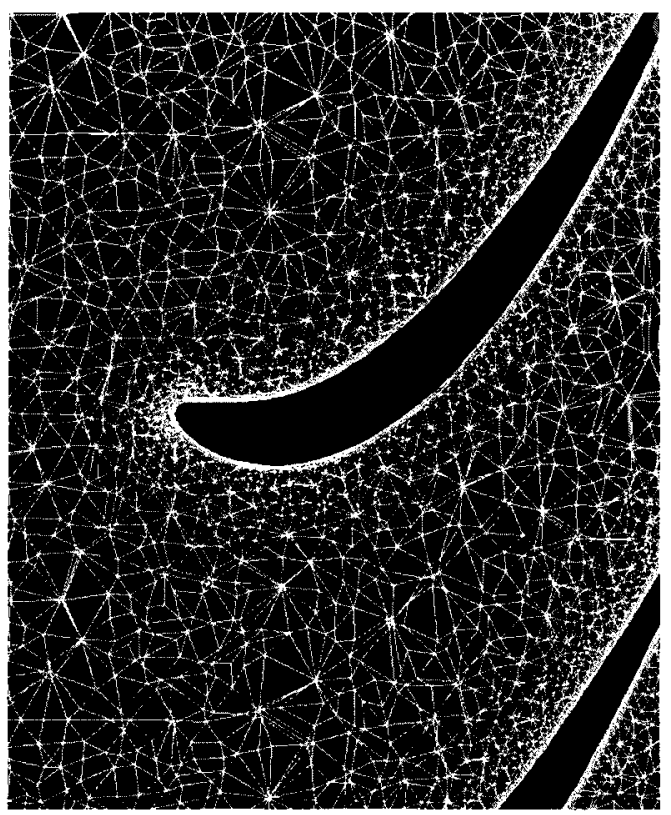

(iii)

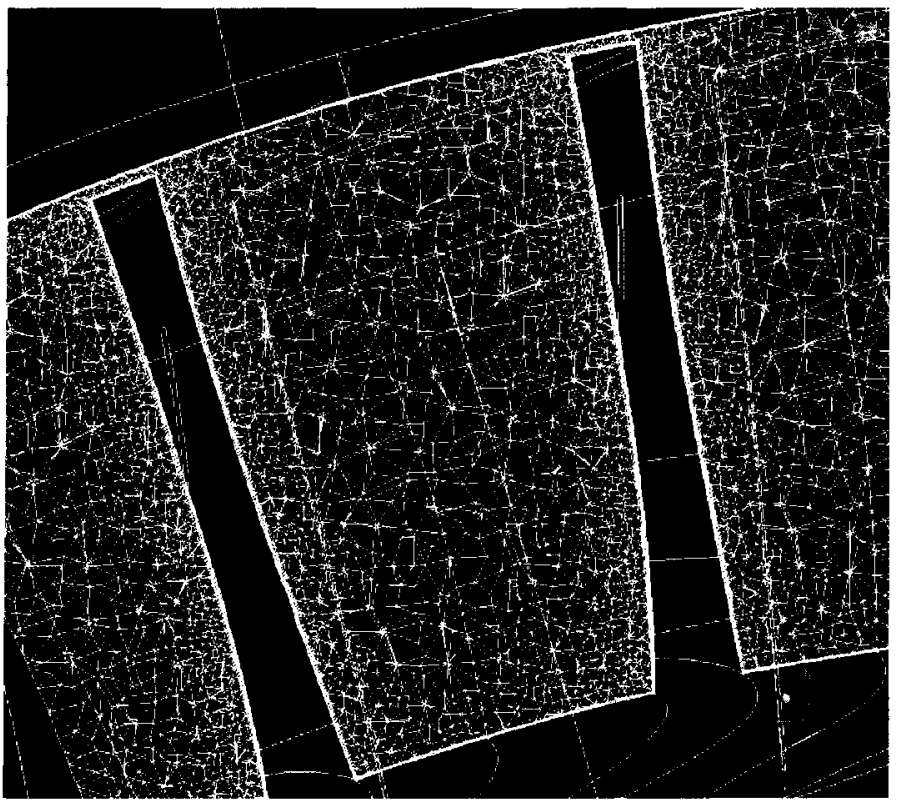

Figure 6.2: Grid density for 220,000 nodes 
(i)

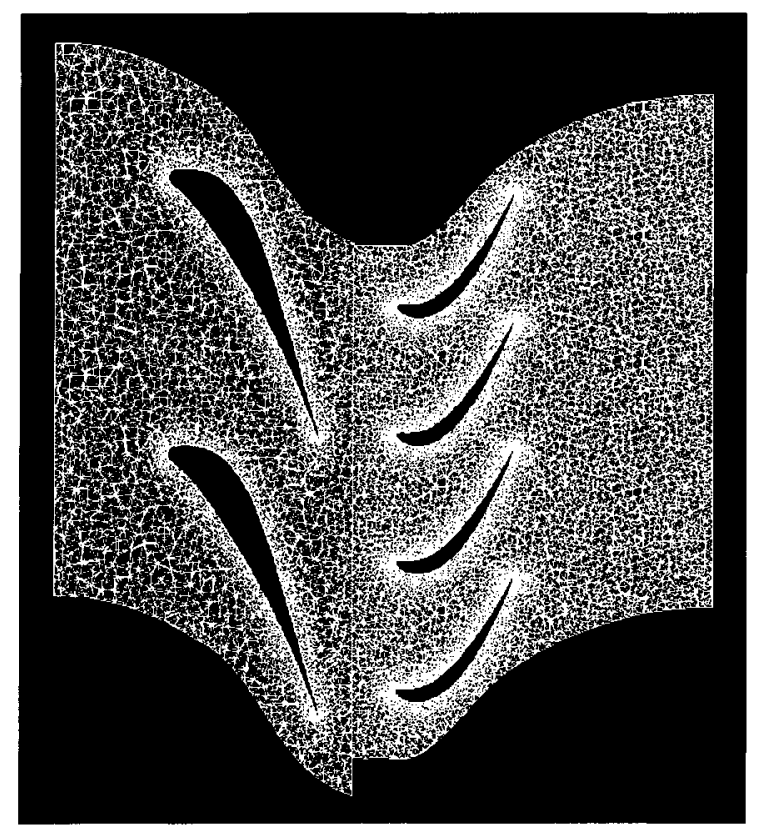

(ii)

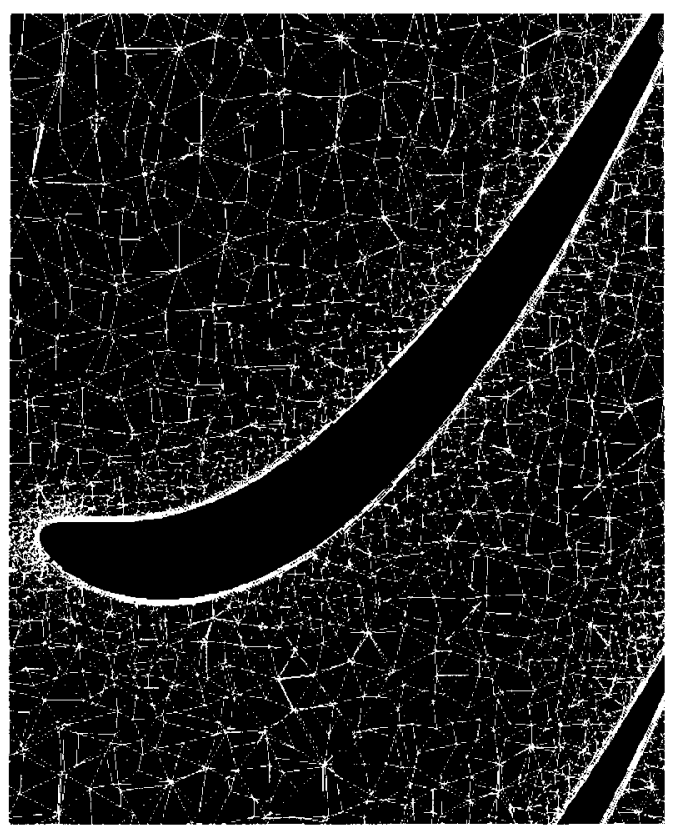

(iii)

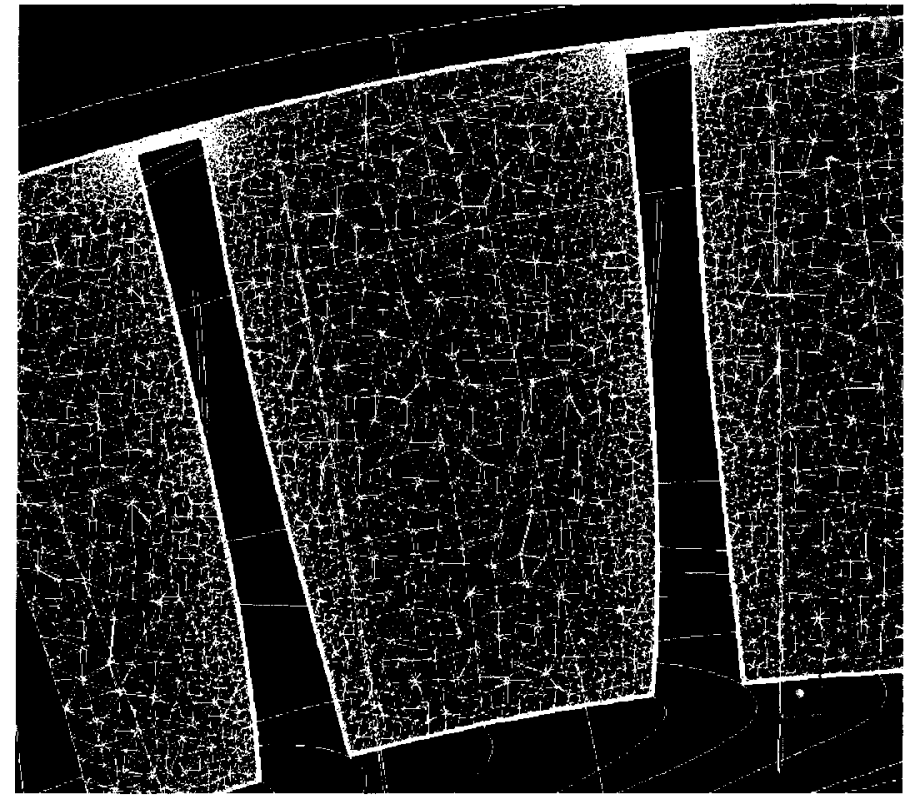

Figure 6.3: Grid density for 490,000 nodes 
Although the overall stage performance of the various simulations is barely affected by the change in grid density, the localized effects are very different. Figure 6.4 illustrates the relative Mach number distribution, or blade loading, around the perimeter of the rotor blade. One can infer that Simulation 1 (110,000 nodes) results in a significantly higher blade loading than Simulation 3 (490,000 nodes). These localized effects are a strong function of the blade inflation layers as well as the grid density immediately adjacent to the rotor blade. Simulation 3 has a much finer mesh and a greater number of inflation layers than both Simulation 1 and 2, resulting in both a higher node count as well as a lower relative Mach number distribution. The additional inflation layers allow for a more gradual change in the velocity distribution from the no-slip wall to the free stream velocity. The sudden drop in Mach number at the trailing edge also occurs earlier in Simulation 3, possibly indicating a small degree of separation.

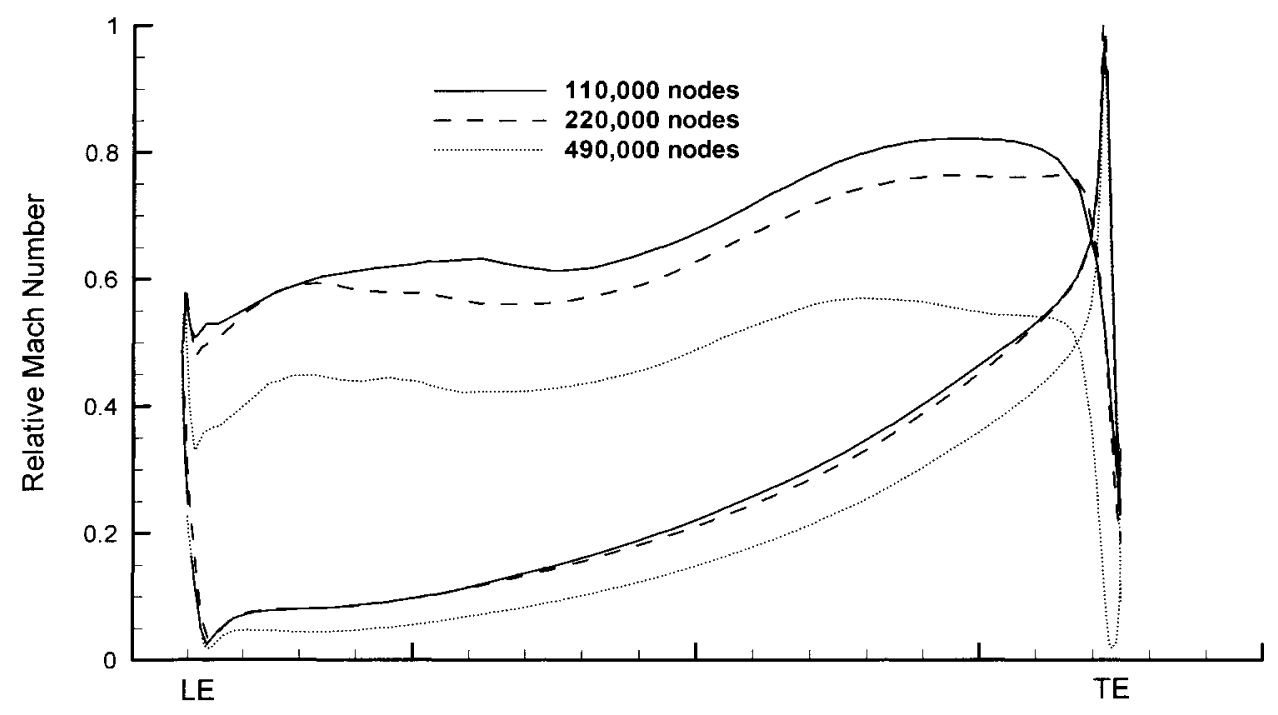

Figure 6.4: Localized effects of the grid refinement study

These localized effects are much more evident in the greater mesh densities. The difference between the overall efficiencies presented in Table 6.2 and the localized blade loading presented here is concerning. If one were to integrate the pressure distribution indicated by blade loading for 490,000 nodes, one would find a much lower torque results on a given blade at its mid span. This in turn should indicate a lower power output and 
thus a lower efficiency. Currently, it is thought that this phenomenon could be attributed to the low aspect ratios being employed by the design, thus contributing to a large variation in the blade loading at the hub and tip sections as a result of strong secondary flows. It is stressed however, that these effects are discussed for interest purposes only, and the lower mesh densities will suffice for the intended purposes of validating the overall stage performance of the design tool. The results presented in the following sections have been generated from meshes comprised of approximately 200,000 nodes.

\subsubsection{Aerodynamic Design Validation - Gas Generator Turbine}

This section is intended to validate the gas generator turbine design as well as the design tool itself. A comparison of the design tool outputs and CFX results is presented first. A very close agreement is found between most of the results. Additional figures are presented to provide additional confidence in the design itself as well as to provide an indication where design improvements may be made. Finally, comparison of the resulting design performance with the initial design specifications is presented.

It should be noted, that an enormous amount of data may be extracted from both the design tool, and the CFX results. Only the data required to perform the design validation and obtain confidence in the design is presented. Table 6.3 presents a summary of the most relevant outputs from the design tool and the CFX simulation. 


\begin{tabular}{|c|c|c|c|}
\cline { 2 - 4 } & Design Tool Output & CFX Results & $\Delta \%$ \\
\hline $\mathrm{T}_{\text {oin }}[\mathrm{K}]$ & 1200 & 1200 & N/A (input) \\
\hline $\mathrm{P}_{\text {oin }}[\mathrm{Pa}]$ & 763,000 & 763,000 & N/A (input) \\
\hline $\mathrm{M}_{\text {in }}$ & 0.38 & 0.17 & 123.5 \\
\hline $\mathrm{T}_{\text {0out }}[\mathrm{K}]$ & 945.2 & 948.9 & -0.4 \\
\hline $\mathrm{P}_{\text {oout }}[\mathrm{Pa}]$ & 243,800 & 256,600 & -5.0 \\
\hline $\mathrm{P}_{\text {out }}[\mathrm{Pa}]$ & 214,400 & 210,000 & N/A (input) \\
\hline $\mathrm{M}_{\text {out }}$ & 0.96 & 1.06 & -9.4 \\
\hline$\alpha_{\text {out }}[$ degrees $]$ & -12 & -10.4 & 15.4 \\
\hline Mass flow $[\mathrm{kg} / \mathrm{s}]$ & 5.507 & 5.74 & -4.1 \\
\hline$\Delta \mathrm{h}_{0}[\mathrm{~kJ} / \mathrm{kg}]$ & -294.5 & -288.8 & 2.0 \\
\hline$\eta[$ total-to-total $]$ & 86.0 & 88.2 & -2.5 \\
\hline Power $[\mathrm{kW}]$ & -1621.8 & -1657.7 & 2.2 \\
\hline
\end{tabular}

Table 6.3: Gas generator turbine validation

A number of points can be raised in light of these results. Most importantly, it can be seen that a close agreement is found between the overall stage performance predicted by the design tool and the full three-dimensional CFX evaluation. The total-tototal isentropic efficiency of the design tool has been predicted within 2.5 percent of the efficiency calculated by CFX. The difference in efficiency may be attributed to the total pressure loss across the stator blade row. In the design tool, a five percent total pressure loss is predicted as a result of the low stator aspect ratio. In the CFX solution however, about three percent is realized. Two factors may contribute to this reduced total pressure loss. First, the blade heights of the stator were significantly modified from the output of the design tool. This was required in order to obtain a manageable pressure loss across the blade row as well as the desired outlet flow angle. It was found in CFX-BladeGen that a converging annulus can obtain a better stator performance than a diverging annulus (for constant axial flow velocity) as initially specified in the design tool. This is a result of the acceleration of the flow being pushed further downstream, ensuring that the boundary layers remain thin in the region where they may tend to separate, reducing the possibility of additional secondary flows. Secondly, the inflation layers on the hub and 
casing upstream of the stator blade row may not have fully resolved the incoming wall boundary layer. This will have an effect on the magnitude of the horseshoe vortices and thus the secondary losses of the stator blade row.

Modification was also required for the various radii of the rotor blades. In this case, a constant blade height was required in order to maintain constant tip clearances and accurately predict the hot running tip gap. The large pressure ratio in the present design leads to high Mach numbers as well as a large area ratio. In order to reduce the annular flare and achieve a constant tip radius, the outlet area was reduced. This had the immediate effect of increasing the velocities and Mach numbers at the rotor exit. Also, a lower exit static pressure was required to obtain the same flow turning and desired work output. A large discrepancy was also indicated between the inlet Mach numbers predicted by the design tool and CFX. This could very well be attributed to the choking of the nozzles in CFX, which is not accounted for in the design tool. The choking nozzles would restrict the mass flow rate and thus reduce the inlet Mach number. Furthermore, variation in the flow angles may also result as the flow attempts to align itself to obtain the required area and Mach number distribution.

To provide further insight into the design, a number of figures created from the CFX results are presented. Figure 6.5 illustrates the Mach number distribution for three span wise locations on the stator blade. The figure identifies a significant incidence effect at the blade leading edge as well as some possible separation and reattachment at the blade trailing edge, particularly at the hub location. 


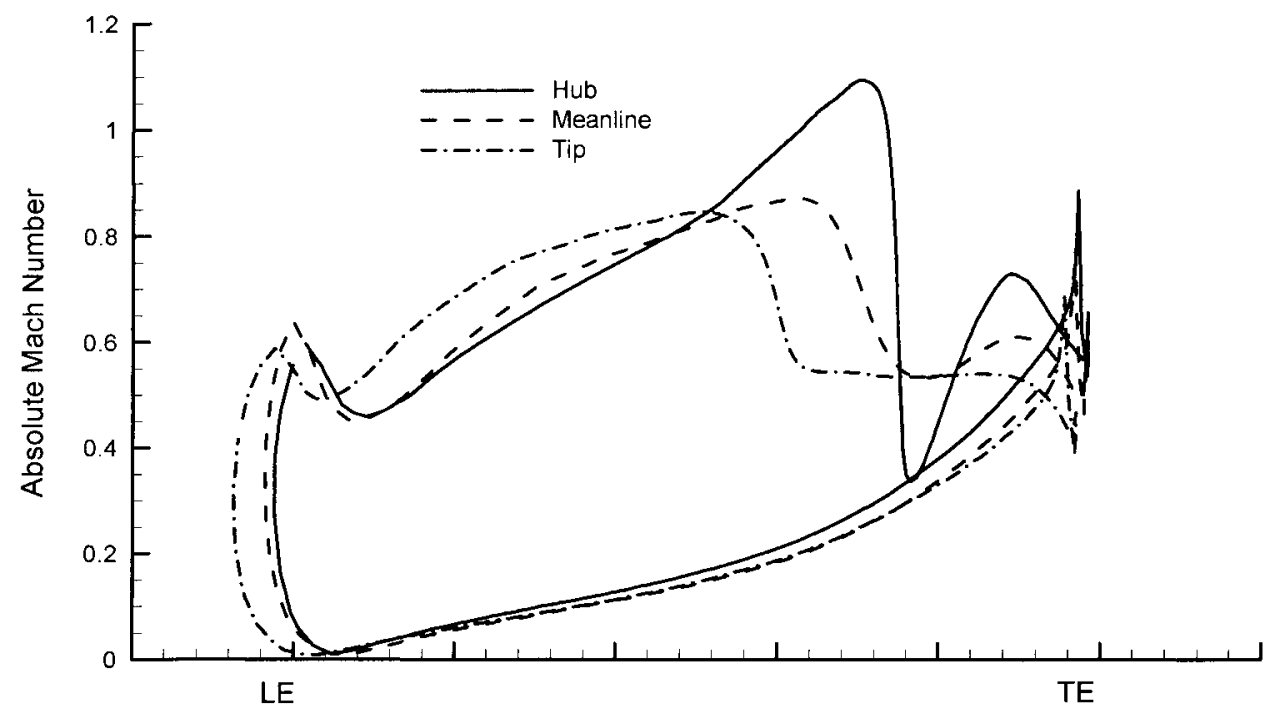

Figure 6.5: Stator blade loading (gas generator turbine)

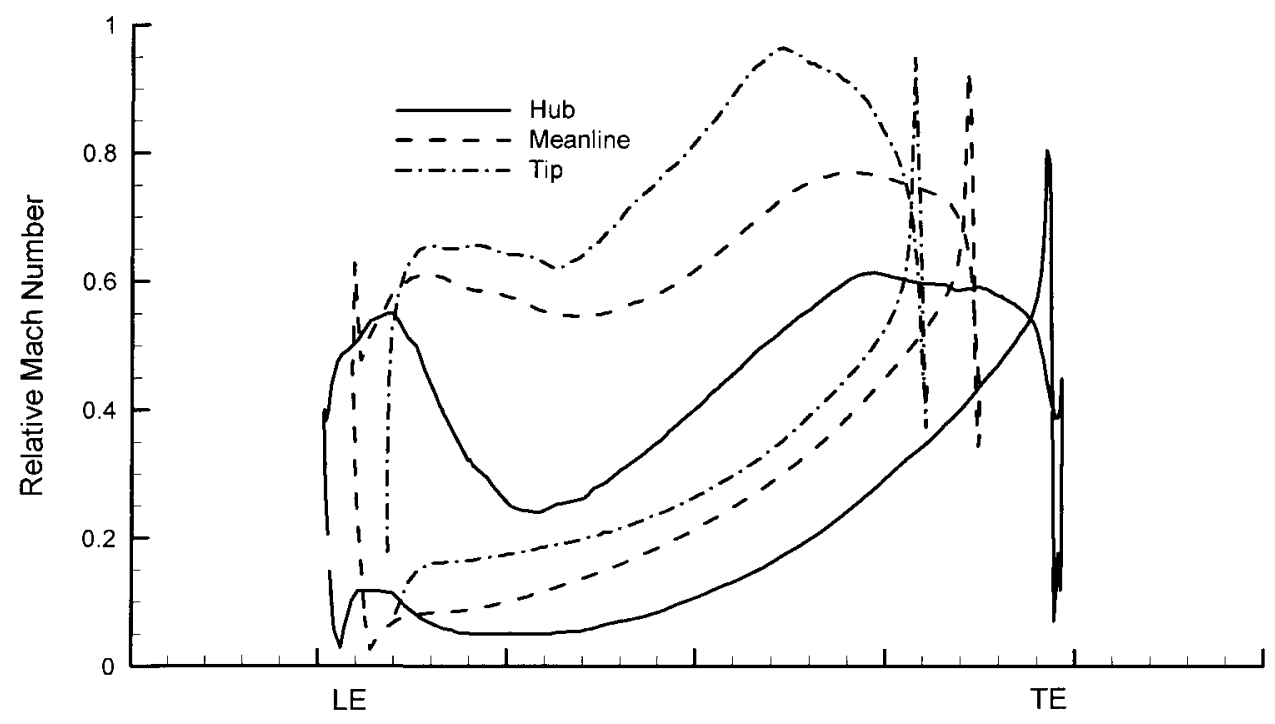

Figure 6.6: Rotor blade loading (gas generator turbine)

Figure 6.6 shows the velocity distribution for a typical aft-loaded turbine blade. There is a large over-acceleration at the blade leading edge, particularly at the hub section. This typically indicates an incidence problem which should be investigated further. The velocity distribution between hub, meanline, and tip is increasing with radial 
distance as expected. A significantly large amount of diffusion is also present near the blade tip. This may be reason for future investigations.

Figure 6.7 displays a contour plot of the Mach number distribution within the stator and rotor blade rows. The discontinuity at the interface is a result of the relative frames of reference. One may note the small size of the trailing edge wakes being shed from each of the blade rows. This may be a result of the inability of the solution to resolve the boundary layers on the blade surfaces. This could have a large implication on the component efficiency and should be investigated further.

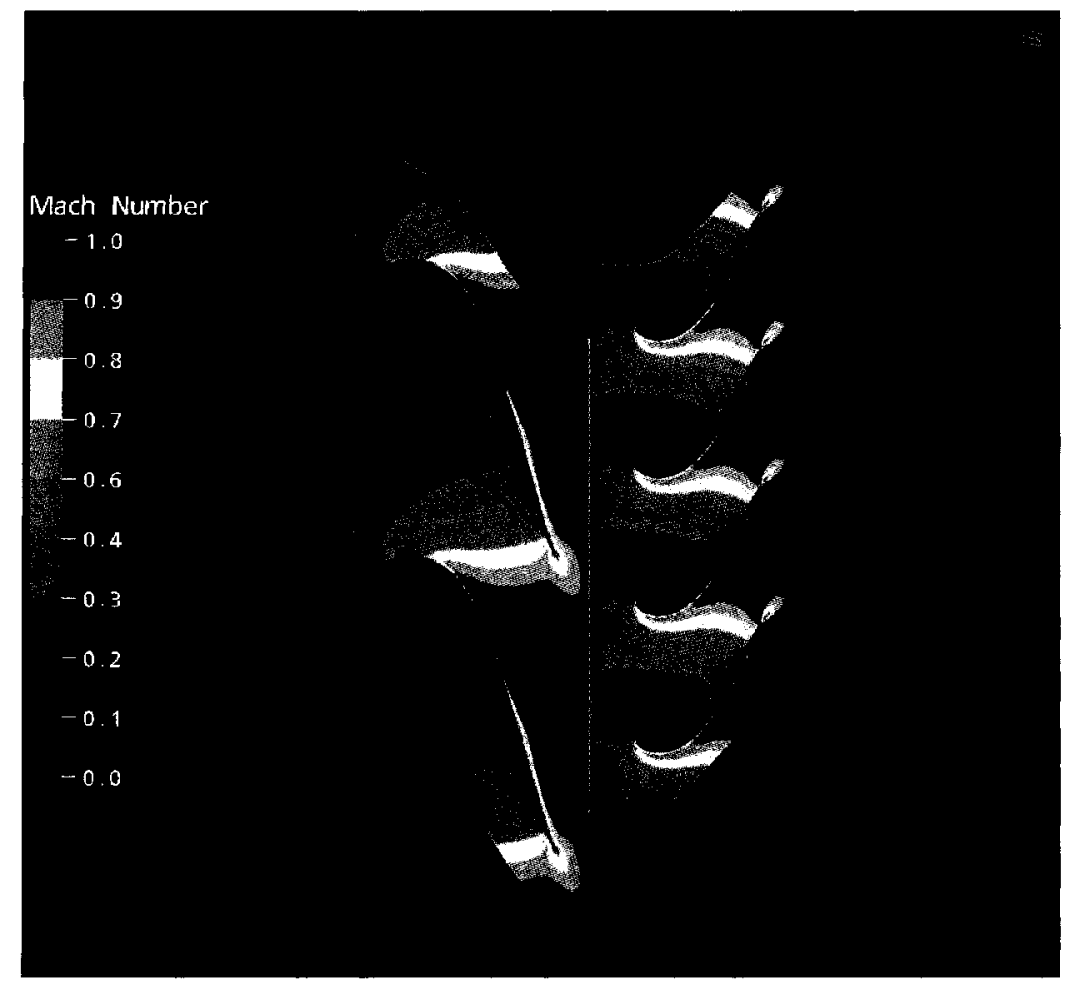

Figure 6.7: Mach number distribution at gas generator turbine mid-span

Figure 6.8 illustrates the effects of the secondary flows present just downstream of the turbine rotor. Figure 6.8 (a) clearly illustrates a large tip vortex and flow characteristics of the contra-rotating vortices. Figure 6.8 (b) illustrates various pressure losses associated with secondary flows, tip clearances, and boundary layer shedding. The local total pressure has been plotted in the relative frame of reference. 
(a)

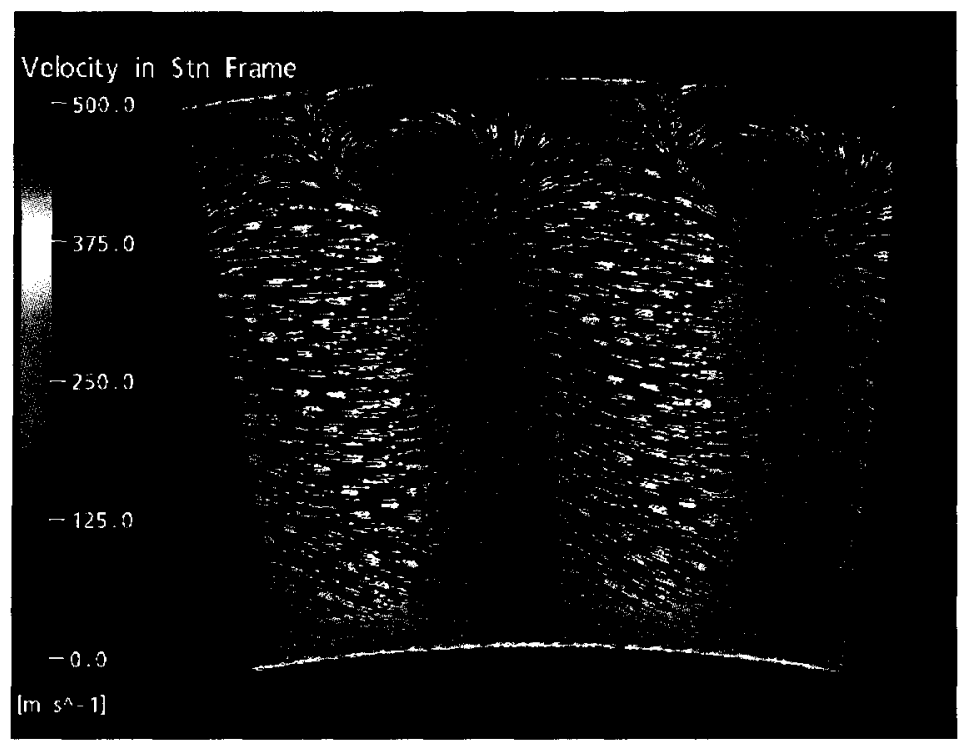

(b)

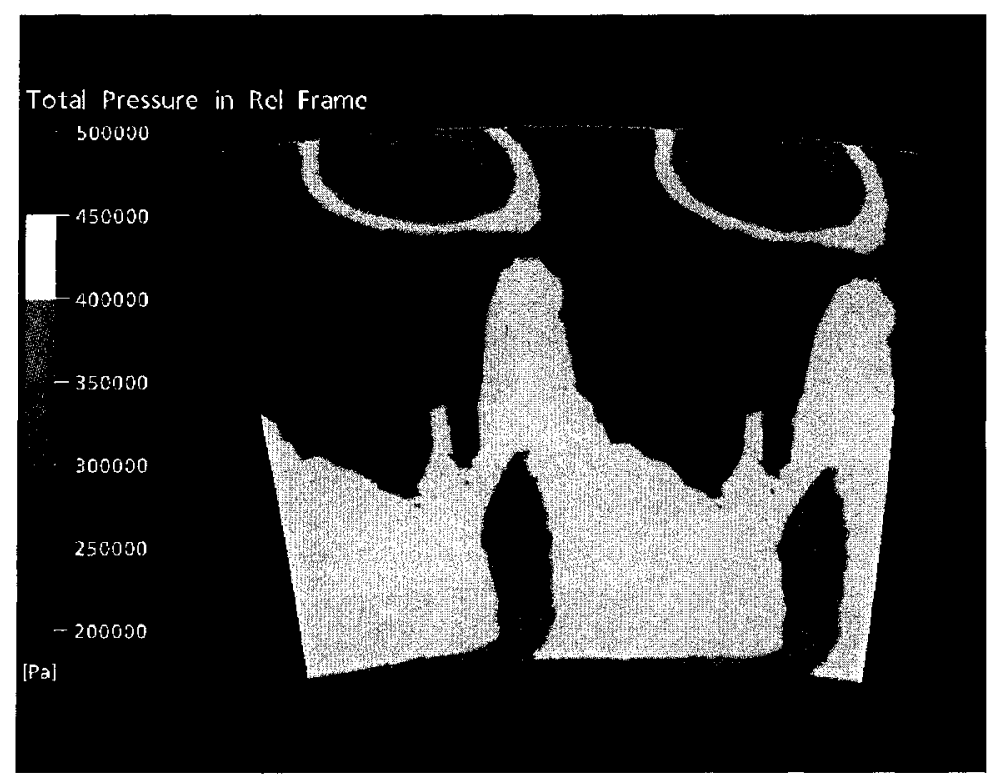

Figure 6.8: (a) Vector diagram and (b) total pressure distribution downstream of gas generator turbine rotor

The mass flow and turbine efficiency maps were also generated using CFX-5. Twelve separate runs were conducted with varying outlet static pressure and rotational speed. The trends shown in Figure 6.9 (a) and (b) are similar to the typical gas turbine characteristics found in literature. These figures indicate the effects of choking at high pressure ratio and a rather flat efficiency curve over a wide range of pressure ratios. 
(a)

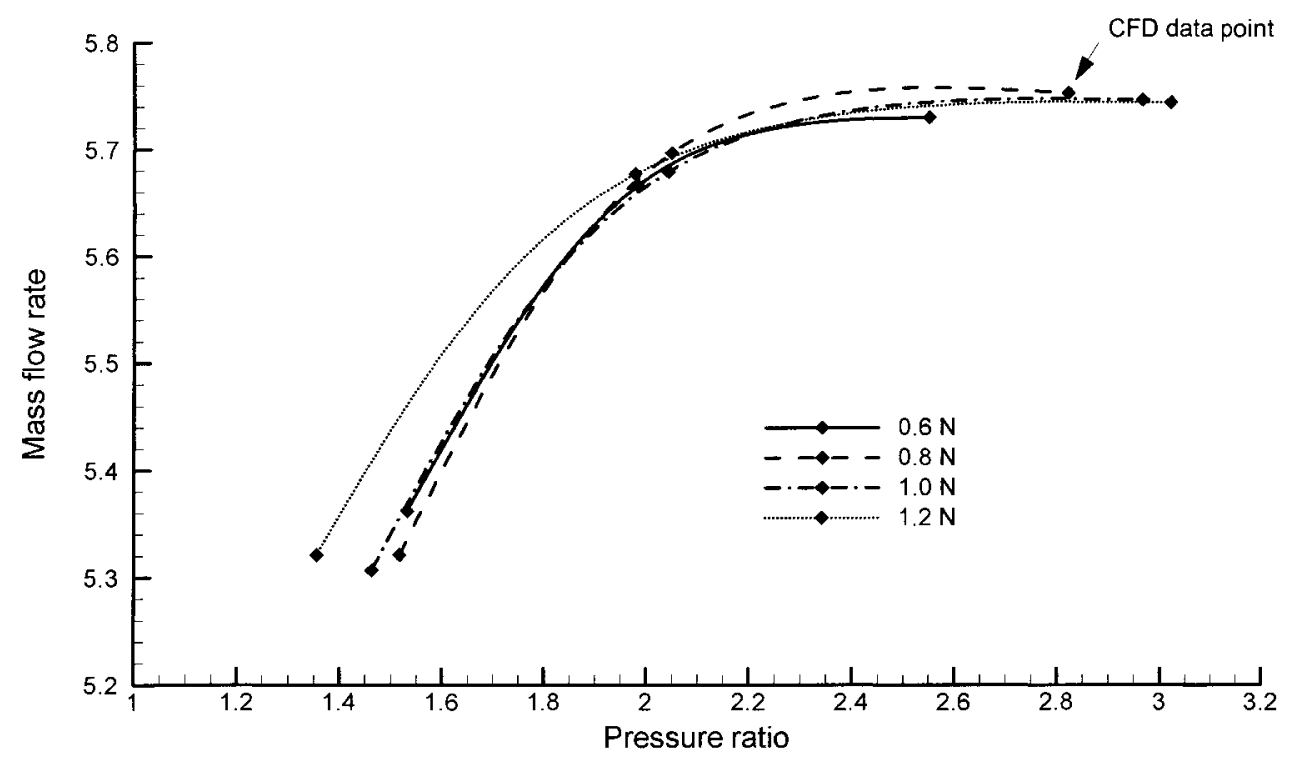

(b)

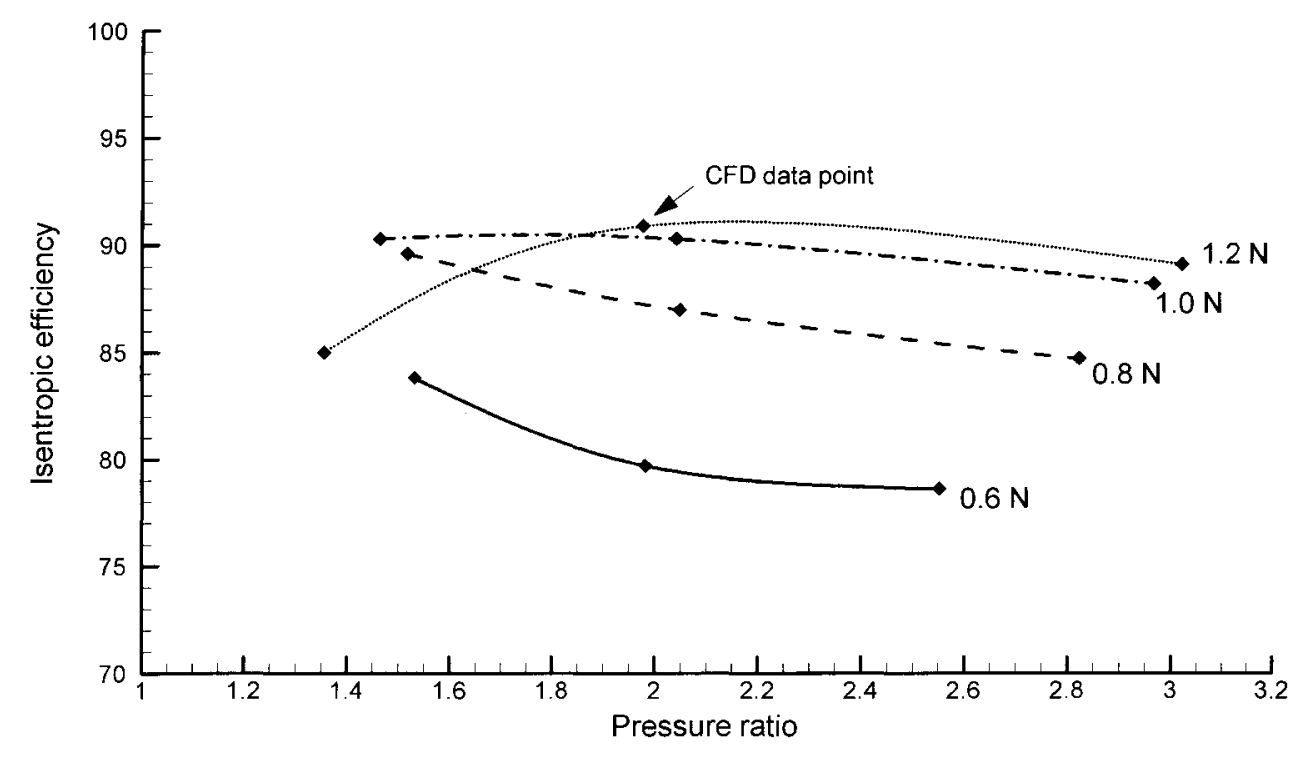

Figure 6.9: Gas generator turbine characteristics

In both instances, the overall performance prediction is slightly lower than desired as a result of the two percent tip clearance used in both the CFX and design tool calculations. The larger tip gap also contributes to a lower specific work output, $\Delta \mathrm{h}_{0}$, in the CFX solution. This is not the case for the design tool as the specific work output is specified. These results are still deemed acceptable since reducing the tip gap by a full percent should in fact increase the efficiency by two whole percentage points, thus 
exceeding the design specifications of the CFX results and increasing the design tool output to approximately 88 percent. Reducing the tip gap will also have the effect of increasing the specific work output of the CFX solution, as required.

\subsubsection{Aerodynamic Design Validation - Power Turbine}

A similar set of results and figures to that presented in the previous section will be used to validate the power turbine design. The results are also used to further validate the aerodynamic performance evaluation conducted by the design tool. Table 6.4 presents the overall performance results obtained from the design tool and the CFD simulations. Once again, the blade hub and tip radii were modified in order to obtain a constant blade tip radius and a convergent stator ring. For this reason, and those discussed for the gas generator design, a significant discrepancy exists between the design tool inlet Mach number and that obtained from the CFD simulation.

The remaining CFD results agreed very well with the outputs of the design tool. The overall stage efficiency and power output calculated by CFX were practically identical to those predicted by the tool. Furthermore, the boundary conditions used in the CFD model also matched those predicted by the design tool outputs. In the earlier case, it was necessary to alter these boundary conditions to obtain the desired work output. These are most likely due to the alterations made to the blade tip radii in the CFD modeling. In addition, the better efficiency comparison may be a result of the larger aspect ratios used in the power turbine design. This indicates that there may be a limit to which the performance prediction correlations can adequately predict the effects of secondary flow due to low aspect ratios. Furthermore, the larger annular flow passage of the power turbine would be less susceptible to alterations than the gas generator passage. 


\begin{tabular}{|c|c|c|c|}
\hline & Design Tool Output & CFX Results & $\Delta \%$ \\
\hline $\mathrm{T}_{\text {Oin }}[\mathrm{K}]$ & 945 & 945 & N/A (input) \\
\hline $\mathrm{P}_{\text {oin }}[\mathrm{Pa}]$ & 255,800 & 255,800 & N/A (input) \\
\hline $\mathrm{M}_{\text {in }}$ & 0.35 & 0.16 & 118.8 \\
\hline $\mathrm{T}_{\text {0out }}[\mathrm{K}]$ & 769.1 & 765.2 & 0.5 \\
\hline $\mathrm{P}_{\text {Oout }}[\mathrm{Pa}]$ & 101,600 & 101,500 & 0.1 \\
\hline $\mathrm{P}_{\text {out }}[\mathrm{Pa}]$ & 92,000 & 90,000 & N/A (input) \\
\hline $\mathrm{M}_{\text {out }}$ & 0.92 & 0.94 & -2.1 \\
\hline$\alpha_{\text {out }}[$ degrees $]$ & 11.3 & 14.4 & -21.5 \\
\hline Mass flow $[\mathrm{kg} / \mathrm{s}]$ & 5.507 & 5.23 & 5.3 \\
\hline$\Delta \mathrm{h}_{0}[\mathrm{~kJ} / \mathrm{kg}]$ & -199.7 & -199.9 & -0.1 \\
\hline$\eta[$ total-to-total $]$ & 89.4 & 89.6 & -0.2 \\
\hline Power $[\mathrm{kW}]$ & 1100 & 1045 & 5.3 \\
\hline
\end{tabular}

Table 6.4: Power turbine validation

Figures 6.10 and 6.11 display the blade loading diagrams of the power turbine stator and rotor. These figures show similar trends to those described in the previous section. Figure 6.10 shows a large over-acceleration around the blade leading edge. Typically, a large over-acceleration is related to incidence effects at the blade leading edge. Since this phenomenon has been a common occurrence throughout both stage designs, it is recommended that alternate incidence correlations be investigated and potentially incorporated in the design tool. 


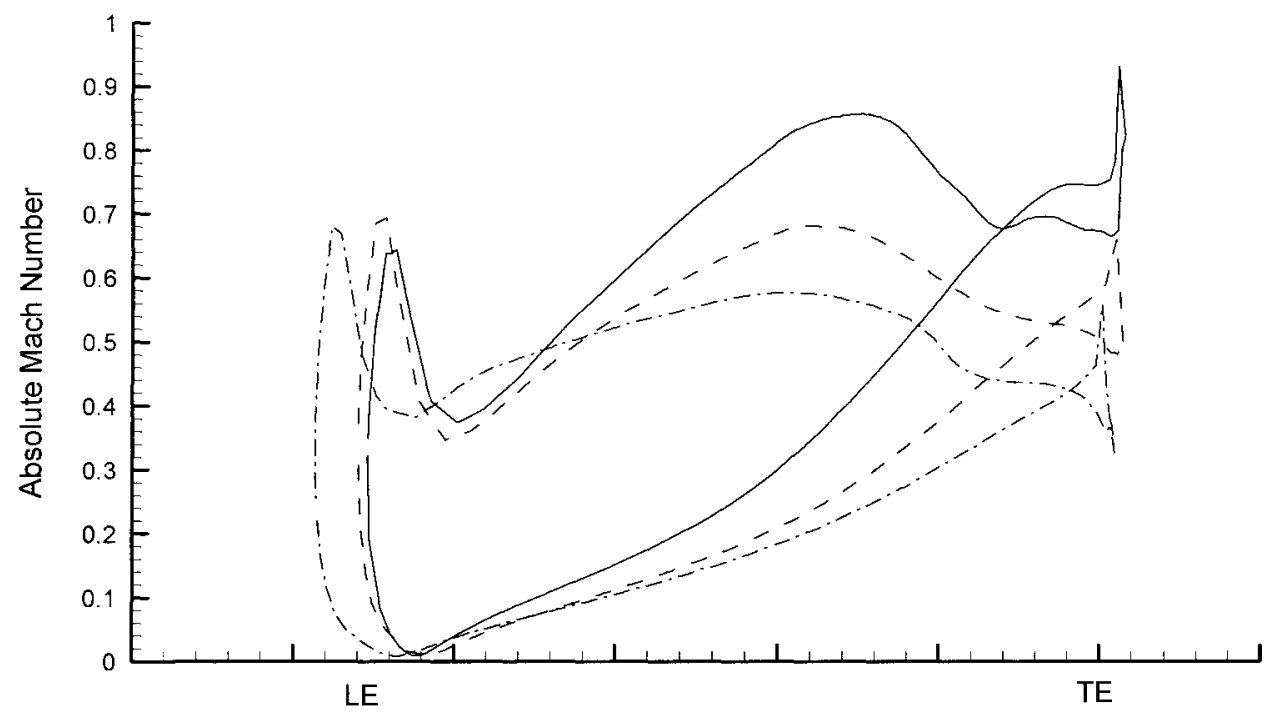

Figure 6.10: Stator blade loading (power turbine)

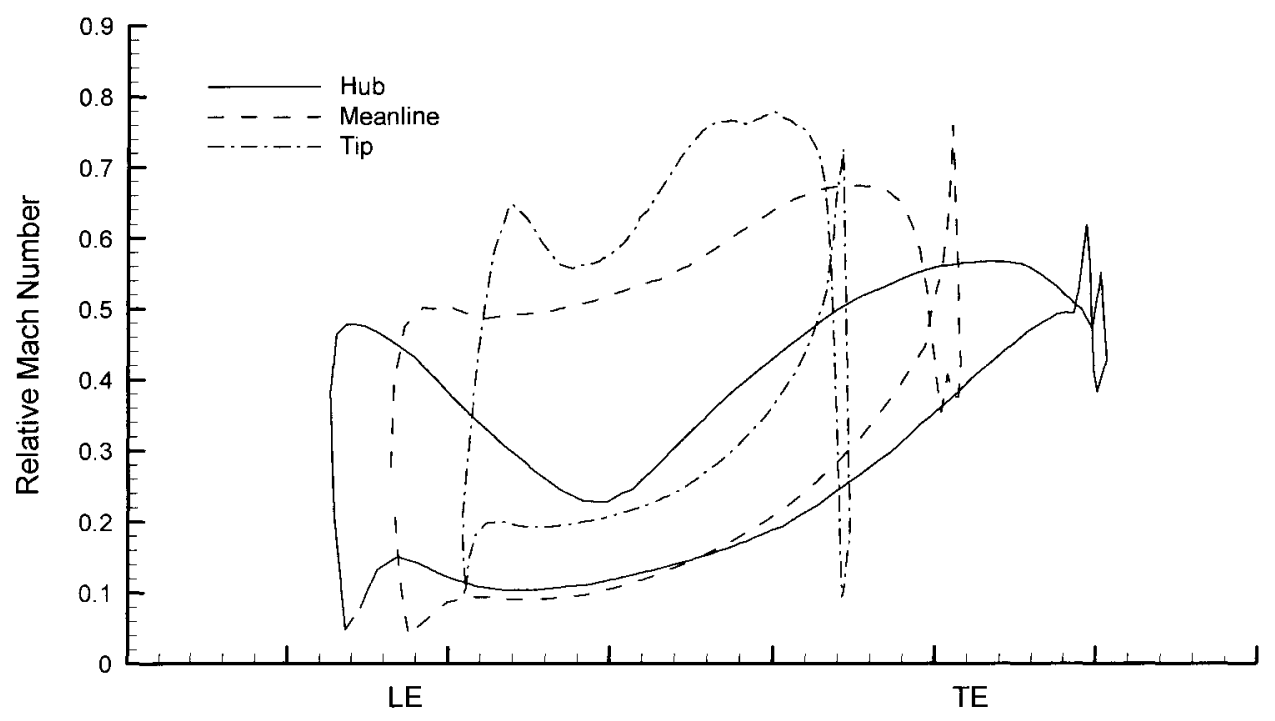

Figure 6.11: Rotor blade loading (power turbine)

Similar to the gas generator turbine validation, the Mach number distribution at 50 percent span is given in Figure 6.12. First, it is observed that there is a change in the direction of blade rotation. This design decision was chosen in order to make use of the swirl component downstream of the first turbine rotor as described previously. Additionally, the reader will note that due to the lower pressure ratio, stage loading, and 
flow coefficient, the blade rows of the power turbine are not fully choked. This provides a performance benefit as it alleviates any sort of shock losses.

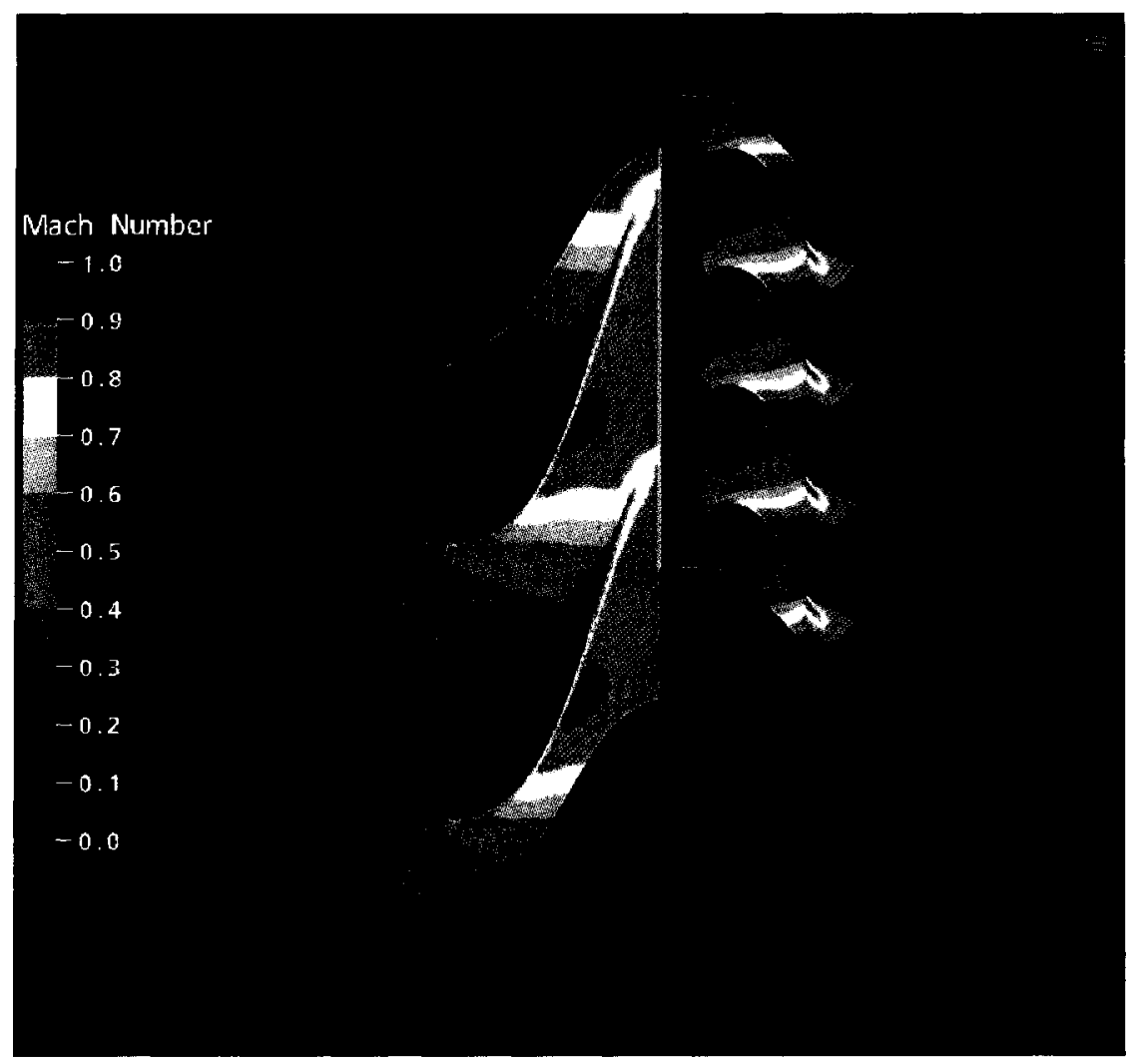

Figure 6.12: Mach number distribution at power turbine mid-span 
(a)

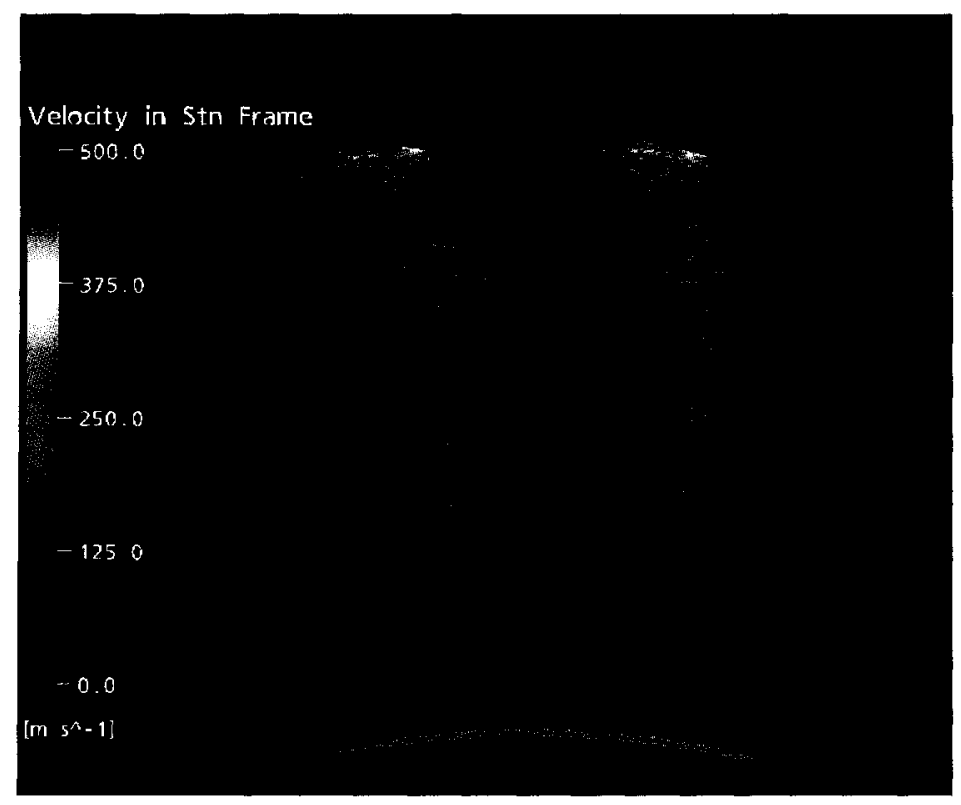

(b)

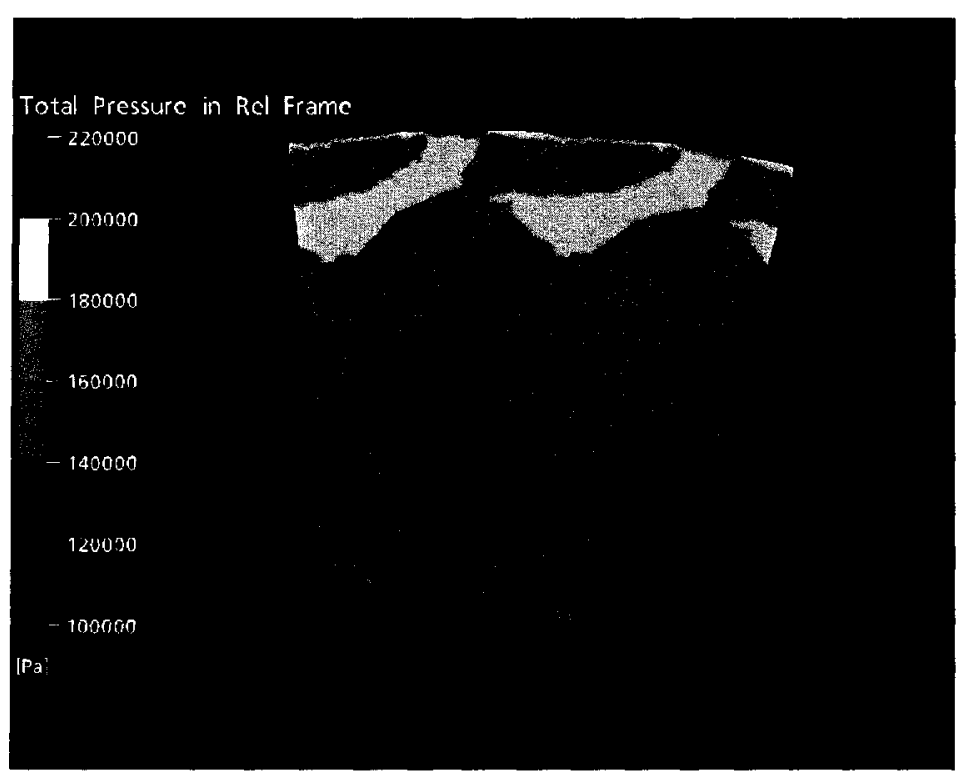

Figure 6.13: (a) Vector diagram and (b) total pressure distribution downstream of power turbine rotor

Figure 6.13 illustrates the flow distribution just downstream of the power turbine rotor. Figure 6.13 (a) is a vector plot showing the large tip clearance vector that is shed downstream of the turbine rotor. Figure 6.13 (b) indicates regions of low total pressure in the relative frame of reference. 
Similar to the gas generator turbine, the turbine performance maps for the power turbine are shown in Figure 6.14. The same trends are noticeable here. It is evident from Figure 6.14 (a) that the flow is not quite choked as was the case in the previous stage.

(a)

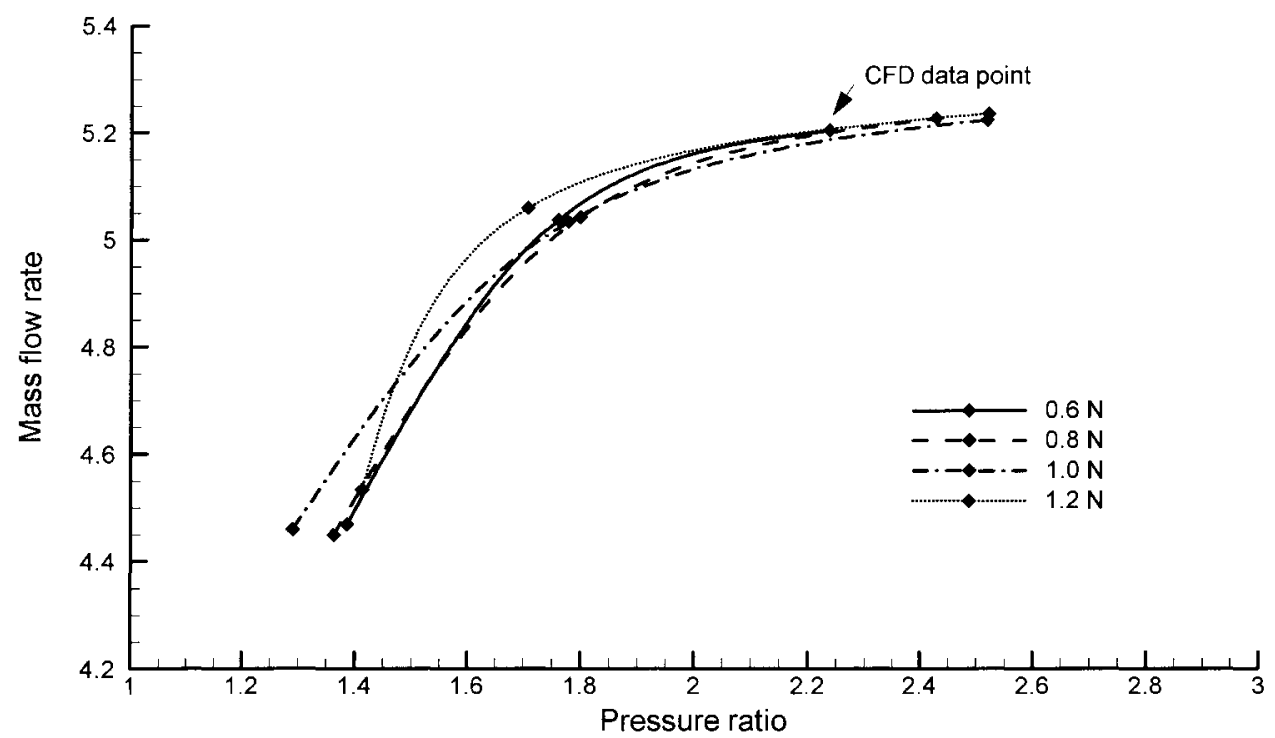

(b)

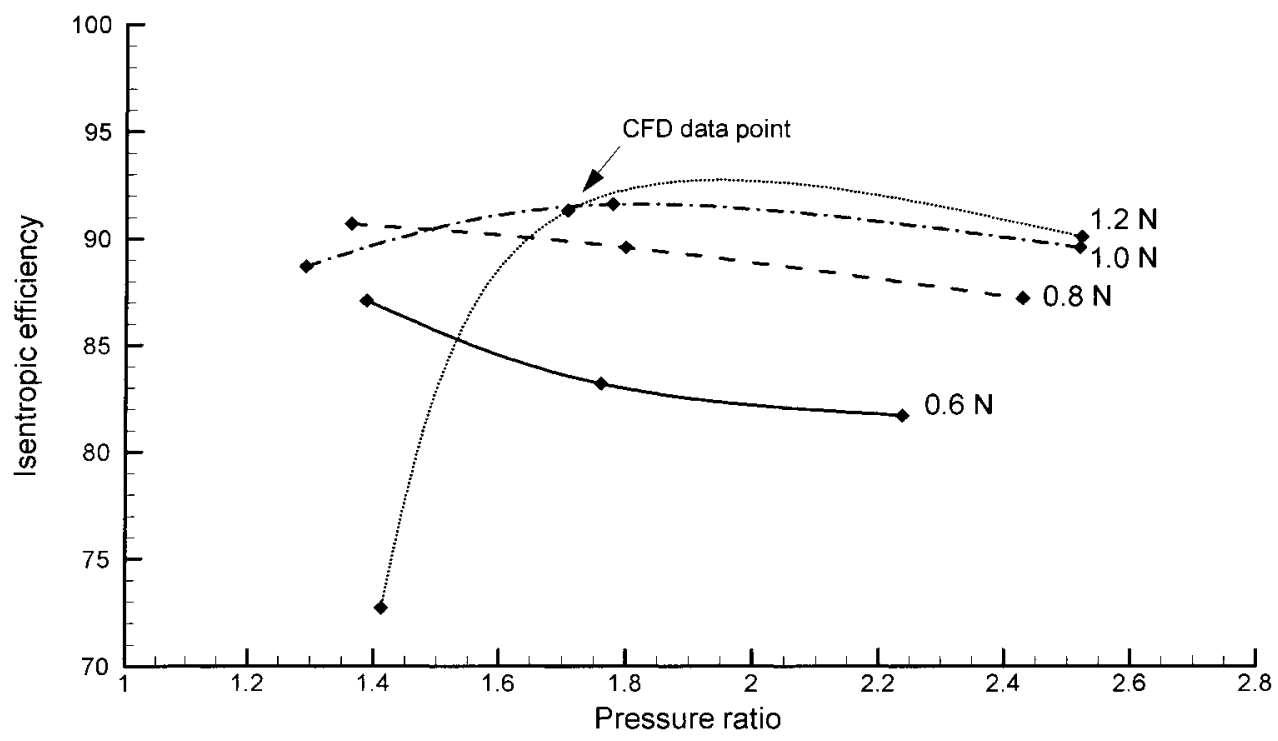

Figure 6.14: Power turbine characteristics 
With respect to the overall design specifications, the aerodynamic performance the power turbine proves to be slightly better than the gas generator turbine. This may be a result of the lower stage loading and pressure ratio found in the power turbine which constitutes a set of much more achievable design specifications, particularly for a single stage. A major deficiency in the current design is the low mass flow rate predicted by the CFD simulation. This may be adjusted by increasing the annular area in future design modifications. 


\subsection{Structural Validation}

A structural validation of the rotor components was also performed. Using the same blade geometry produced by CFX-BladeGen, structural analyses were conducted. For the design tool validation, only an inertial analysis was required. As with the aerodynamic design, additional simulations were also carried out to gain further confidence in the proposed design. These additional simulations consisted of thermal and vibration analyses. The results of all the simulations will be presented in Sections 6.2 .2 and 6.2.3.

\subsubsection{Settings}

ANSYS 9.0 is a complex software tool used primarily for structural evaluation of a variety of engineering components. The software contains numerous options and methods for conducting various analyses. The settings used in each of the analyses are presented in Appendices D5 through D8. This section will provide a summary of each of these settings along with a brief discussion.

The first, and perhaps the most important, setting in ANSYS 9.0 is the selection of element type. ANSYS 9.0 has over 100 various element types to choose from. These elements may be beam, plane, shell, solid, etc. The element type will have a direct impact on the size and computational requirements of the solution. Although they are more computationally demanding, Solid 95 elements were selected for the structural modeling. Solid 95 is a high order, 20-node, three-dimensional solid element. It can tolerate irregular shapes without as much loss in accuracy as its lower order, 8-node counterpart. Similarly, the Solid 90 element was selected for the thermal analysis. This element is a high order, 20-node, three-dimensional thermal element. These elements were chosen primarily due to the complex curvature of the blade shapes. Solutions with the lower order elements were attempted; however, the complex blade curvature resulted in a variety of meshing errors. 
The next group of settings involved the specification of material properties. For the Young's Modulus and Poisson's Ratio, isotropic linear elastic property values were entered. Isotropic values were also entered for the thermal expansion coefficient as well as the thermal conductivity. The properties shown in Table 6.5 reflect the values entered into ANSYS 9.0 for the chosen material, NIMONIC alloy 115. It should be noted here, that ANSYS 9.0 is unitless; therefore, it is essential to ensure all material properties are consistent. For the given set of material properties, all of the results will be in $[\mathrm{N}],[\mathrm{mm}]$, $[\mathrm{s}]$, and $[\mathrm{K}]$.

\begin{tabular}{|c|c|c|}
\hline \multicolumn{3}{|c|}{ NIMONIC alloy 115} \\
\hline Property [Units] & Designation & Value \\
\hline Density $\left[\mathrm{Ns}^{2} / \mathrm{mm}^{4}\right]$ & DENS & $8.19 e-9$ \\
\hline Young's Modulus [ $\left.\mathrm{N} / \mathrm{mm}^{2}\right]$ & EX & 179,530 \\
\hline Poisson's Ratio & PRXY & 0.317 \\
\hline $\begin{array}{l}\text { Mean Coefficient of Linear Thermal } \\
\text { Expansion }\left[10^{-6} / \mathrm{K}\right]\end{array}$ & ALPX & 14.4 \\
\hline Thermal Conductivity [N/sK] & $k X X$ & 20 \\
\hline
\end{tabular}

Table 6.5: ANSYS material properties and units

Whenever possible, a cyclic constraint should be used. This greatly reduces the computational efforts of the model. Cyclic constraints were used in both the inertial and thermal simulations. Since only the blade was modeled in the vibration analysis, a cyclic constraint was not necessary.

Numerous options exist for mesh generation. For the majority of the meshes used in the analyses, a smart sizing in the range of 4-6 was selected. Unfortunately, meshing in ANSYS 9.0 is not a simple task; it requires patience and practice. Further details have been given in Section 5.4 as well as Appendix D5. 
In the cases that were evaluated, no further options were explored. The remainder of the settings are documented in Appendices D6, D7, and D8. For completeness, they are summarized in Tables 6.6 through 6.8 .

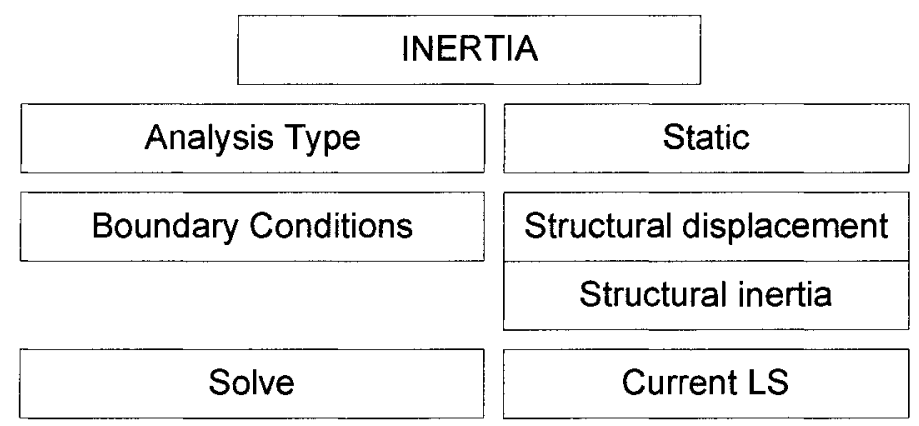

Table 6.6: Summary of inertia analysis settings

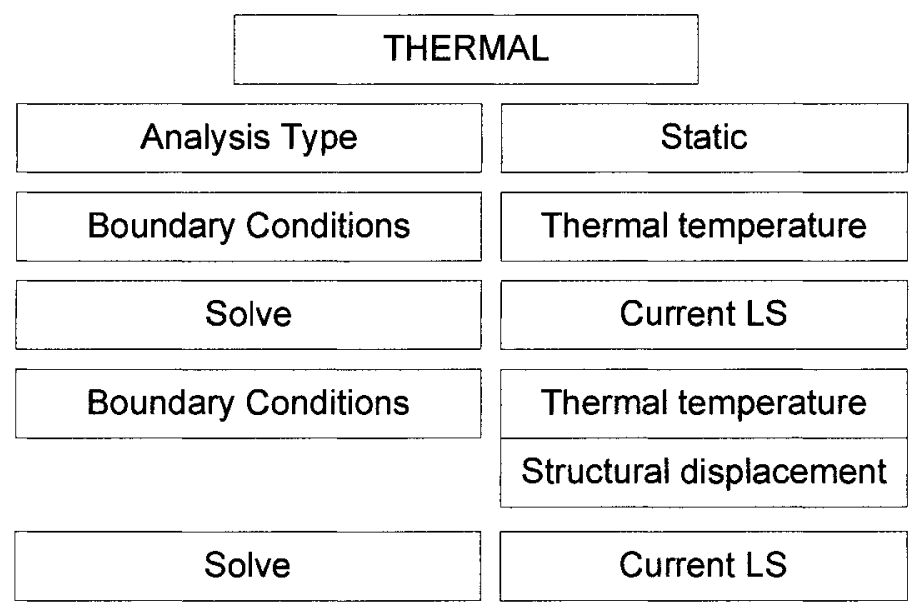

Table 6.7: Summary of thermal analysis settings 


\begin{tabular}{|c|c|}
\hline \multicolumn{1}{c|}{} & VIBRATION \\
\hline Analysis Type & Modal \\
\hline Analysis Options & Block Lanczos extraction method \\
\hline & Specify number of modes to extract \\
\hline Calculate element results \\
\hline Boundary Conditions \\
\hline Solve \\
\hline
\end{tabular}

Table 6.8: Summary of vibration analysis settings

\subsubsection{Structural Design Validation - Gas Generator Turbine}

This section will present a comparison of the results obtained for the structural evaluation conducted by the design tool and ANSYS 9.0. Using an inertial analysis, the blade root stresses and von Mises stress distribution are predicted by ANSYS 9.0. These results are then compared to those obtained in the preliminary design tool. This will complete the structural validation of the stress components. Selected results from the thermal and vibration analyses are then presented and discussed.

The computational mesh used in the inertial and thermal analyses is shown in Figure 6.15. A very fine mesh was required at the blade leading and trailing edges to resolve the highly curved surfaces. Although Figure 6.15 shows five blades, it should be noted that a cyclic constraint was used for a single blade and rotor section. The results are presented as a cyclic expansion of five identical solutions. 


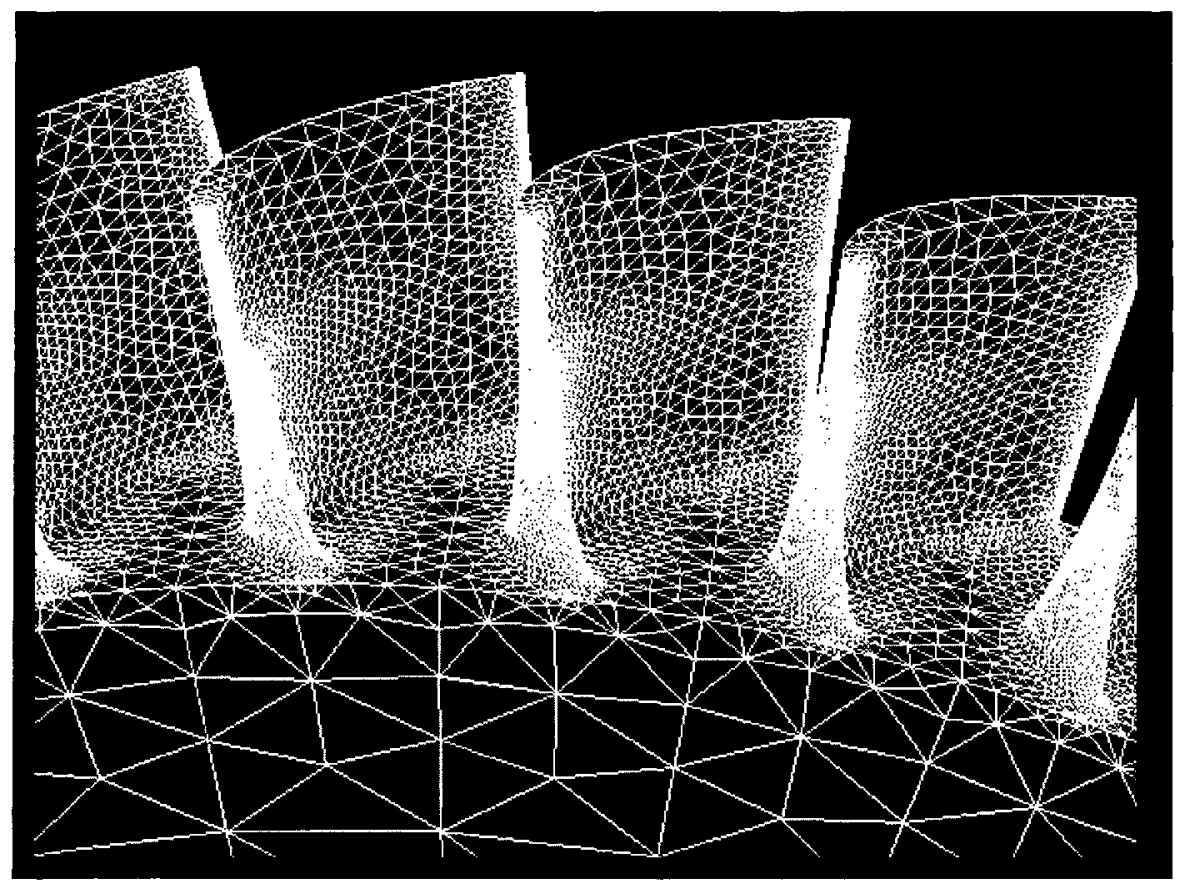

Figure 6.15: Gas generator turbine rotor finite element mesh

Figures 6.16 and 6.17 present the von Mises stress distributions for the gas generator turbine rotor. Figure 6.16 shows the overall stress distribution, indicating the maximum stress at the rotor bore. In fact, the largest calculated stress was found at the blade root trailing edge. This is a result of the very thin trailing edges used to obtain an optimum aerodynamic efficiency. As a high stress in this region is not desired, it is suggested that the trailing edge be thickened slightly, to reduce the stresses in this area. It is also advised to enlarge the fillet radius between the blade root and rotor hub. This will result in a more even stress distribution around the blade profile at the hub, see Figure 6.17. Figure 6.16 also gives an indication of the radial stress distribution within the rotor itself. Using the ANSYS query selection tool, the stress distribution at specific radii was obtained. These results are plotted and compared to those obtained from the preliminary design tool in Figure 6.18. 


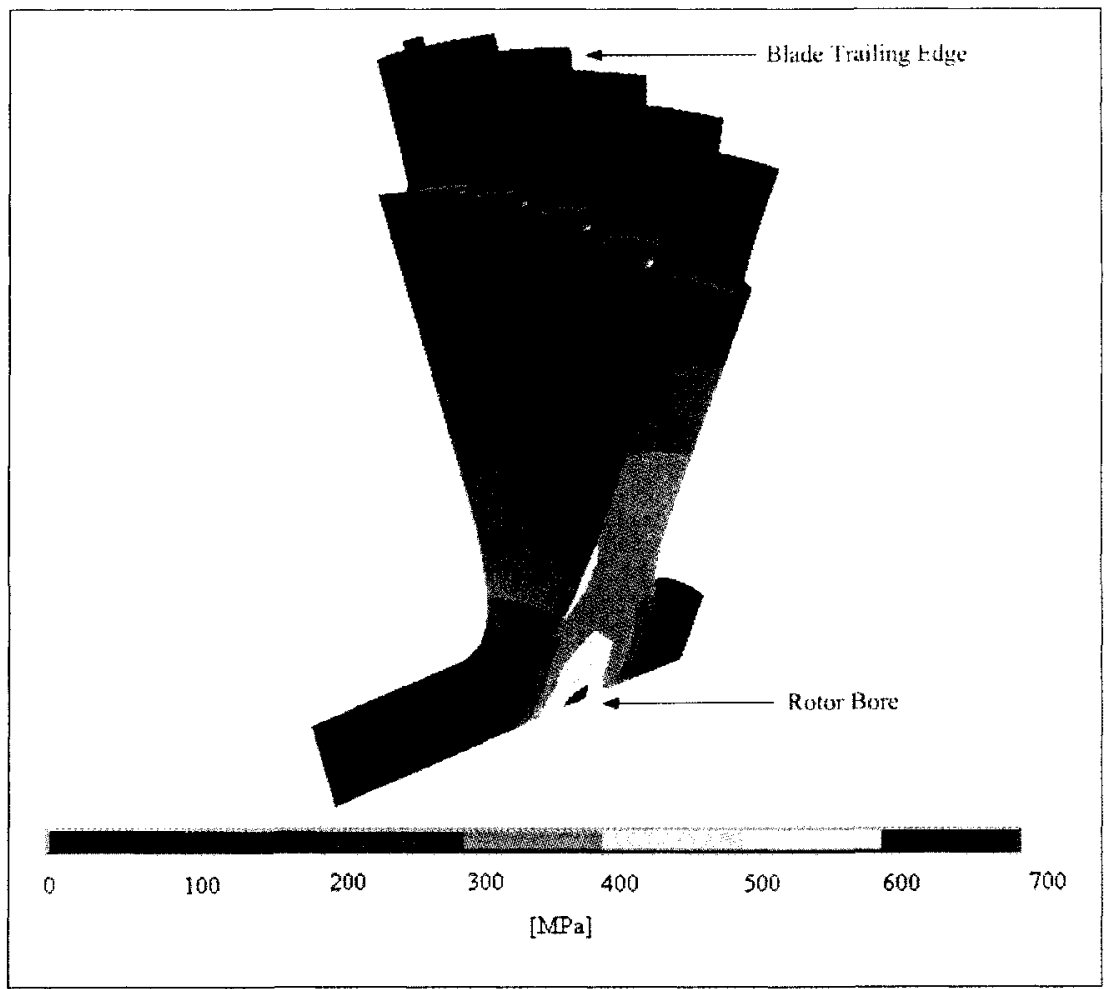

Figure 6.16: Rotor von Mises stress distribution (gas generator turbine)

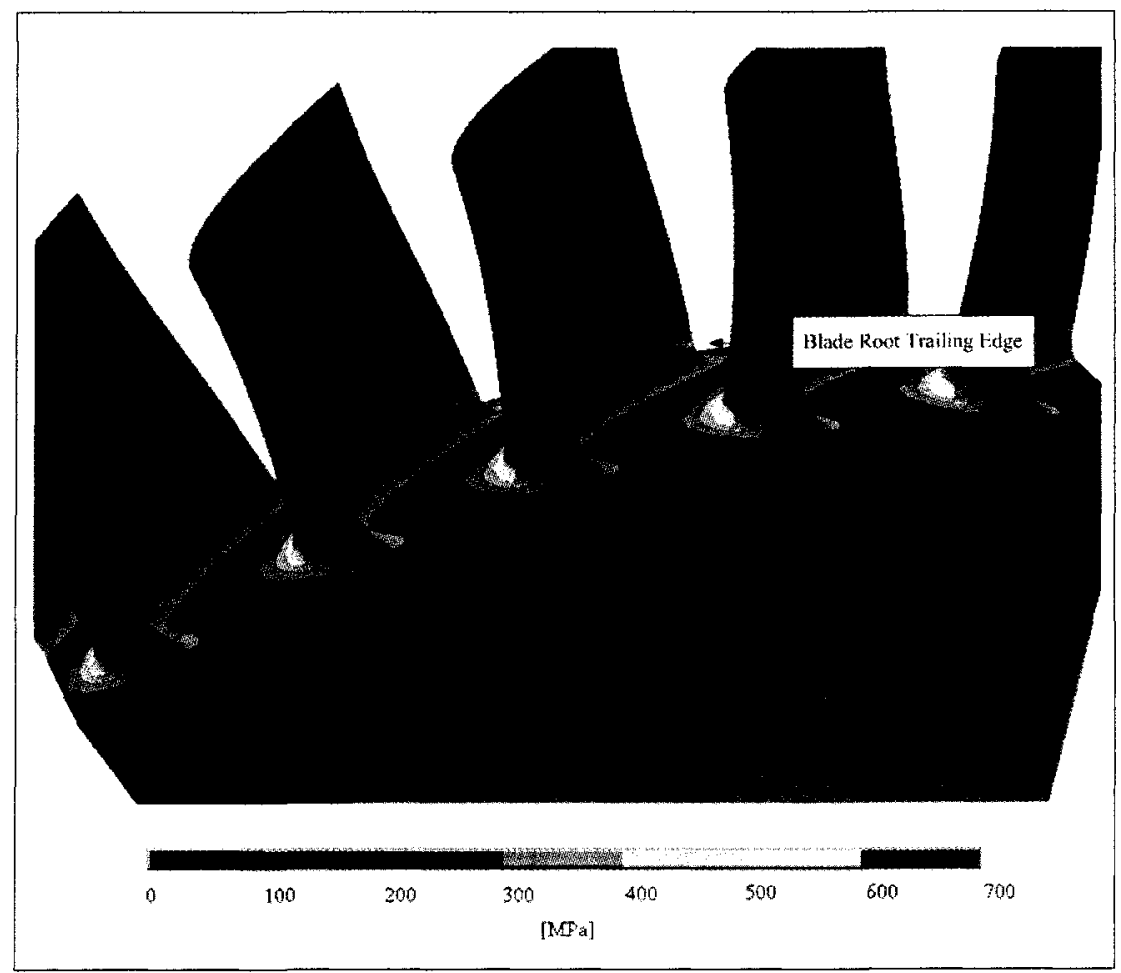

Figure 6.17: Blade von Mises stress distribution (gas generator turbine) 


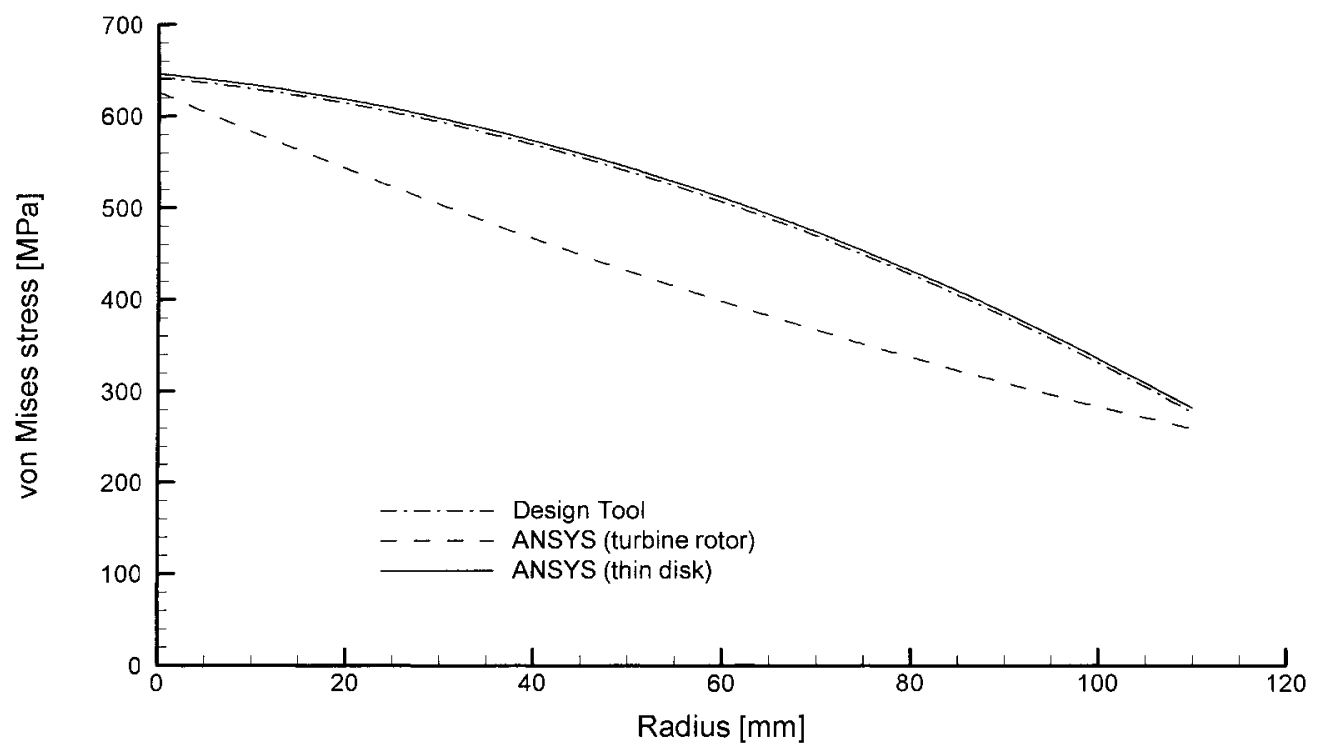

Figure 6.18: Rotor stress distribution - ANSYS vs design tool

The accuracy of the design tool at the extremities of the graph is evident. Clearly, the design tool is capable of accurately predicting the maximum and minimum stresses in the turbine rotor. Discrepancies arise due to the assumption that the gas turbine rotor is a uniformly thin disk. By this assumption, it is also assumed that plane stress exists, so the radial and hoop stresses are constant through the thickness. From Figure 6.16, it can be seen that this is not the case. This can be attributed to the additions of the shaft and fillet in the ANSYS model which were not accounted for in the preliminary design tool. Due to the observed variation in the trends between the results of the design tool and the ANSYS turbine rotor simulation, an additional ANSYS simulation was conducted to prove the validity of the ANSYS modeling processes. A uniform disk with the same dimensions, operational speed, and rim loading as that analyzed by the design tool was simulated. The ANSYS results of the von Mises stress distribution for the uniform disk are indicated by the solid line in Figure 6.18. Clearly, these results are consistent as they follow nearly the same line that is predicted by the design tool.

Using the same computational mesh described before, a thermal analysis was conducted using ANSYS 9.0. This simulation was conducted to obtain a preliminary estimate for the thermal stresses and thermal growth of the turbine rotor. Using only the 
estimated metal temperatures at the blade leading and trailing edges, the temperature distribution was calculated, as shown in Figure 6.19. Currently, these results do not take into account disk cooling or convective heat transfer.

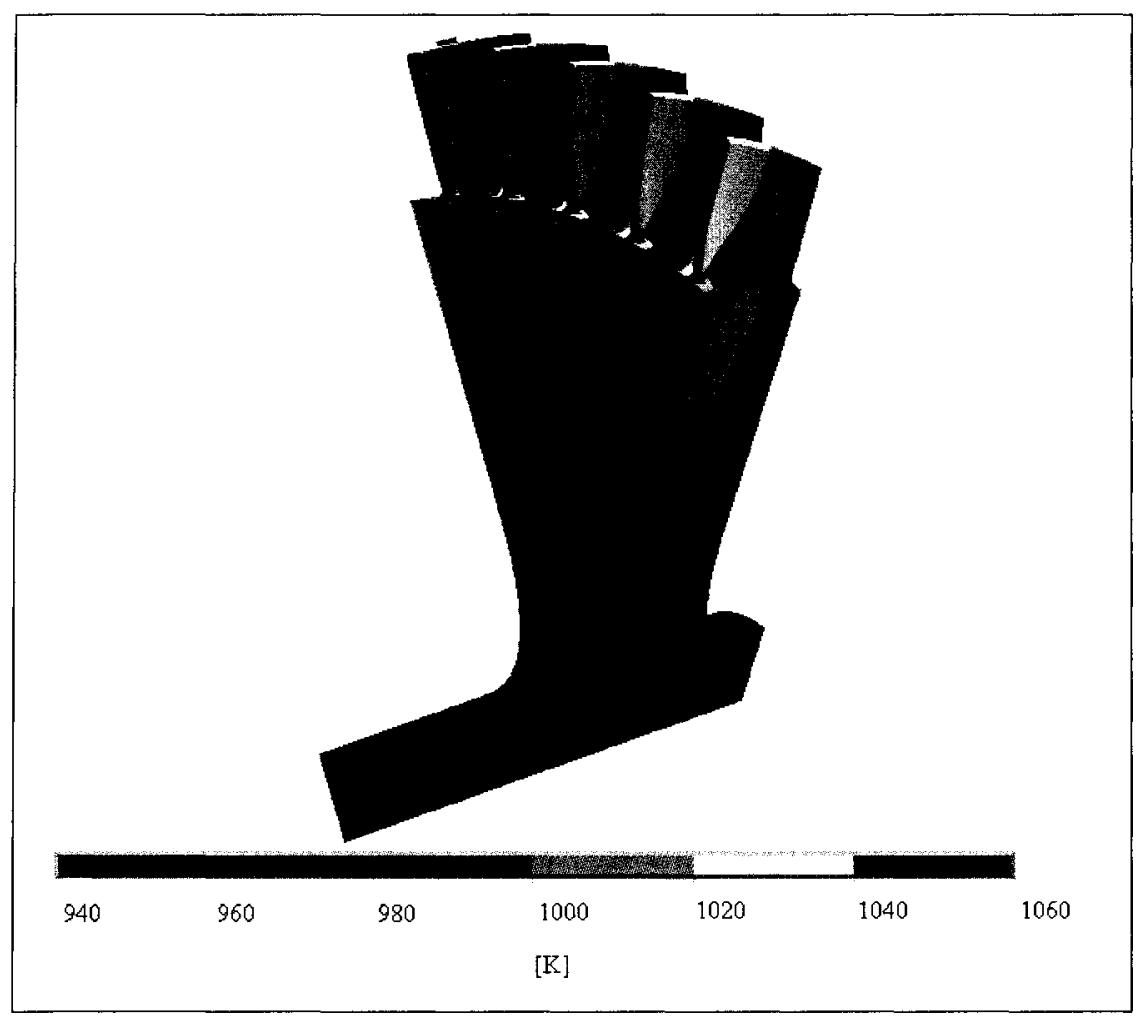

Figure 6.19: Gas generator turbine rotor temperature distribution

This temperature difference results in a maximum thermal stress of approximately $100 \mathrm{MPa}$ at the blade root, near the blade leading edge. This is small in comparison with the results obtained due to the centrifugal loading. The important result obtained in this analysis is the thermal growth of the rotor. The thermal growth is illustrated in Figure 6.20. As one can see, the blade tip grows by approximately $2 \mathrm{~mm}$ (about 7 percent) as a result of the thermal effects. It should be noted, however, that the blade tip growth accounts for the growth of the rotor disk as well. When combined with the radial growth of the centrifugal loading, this total growth is approximately $2.2 \mathrm{~mm}$. Note that this analysis has not taken the effects of creep into account. Also note that this value may be reduced if disk cooling and convection effects are accounted for. 


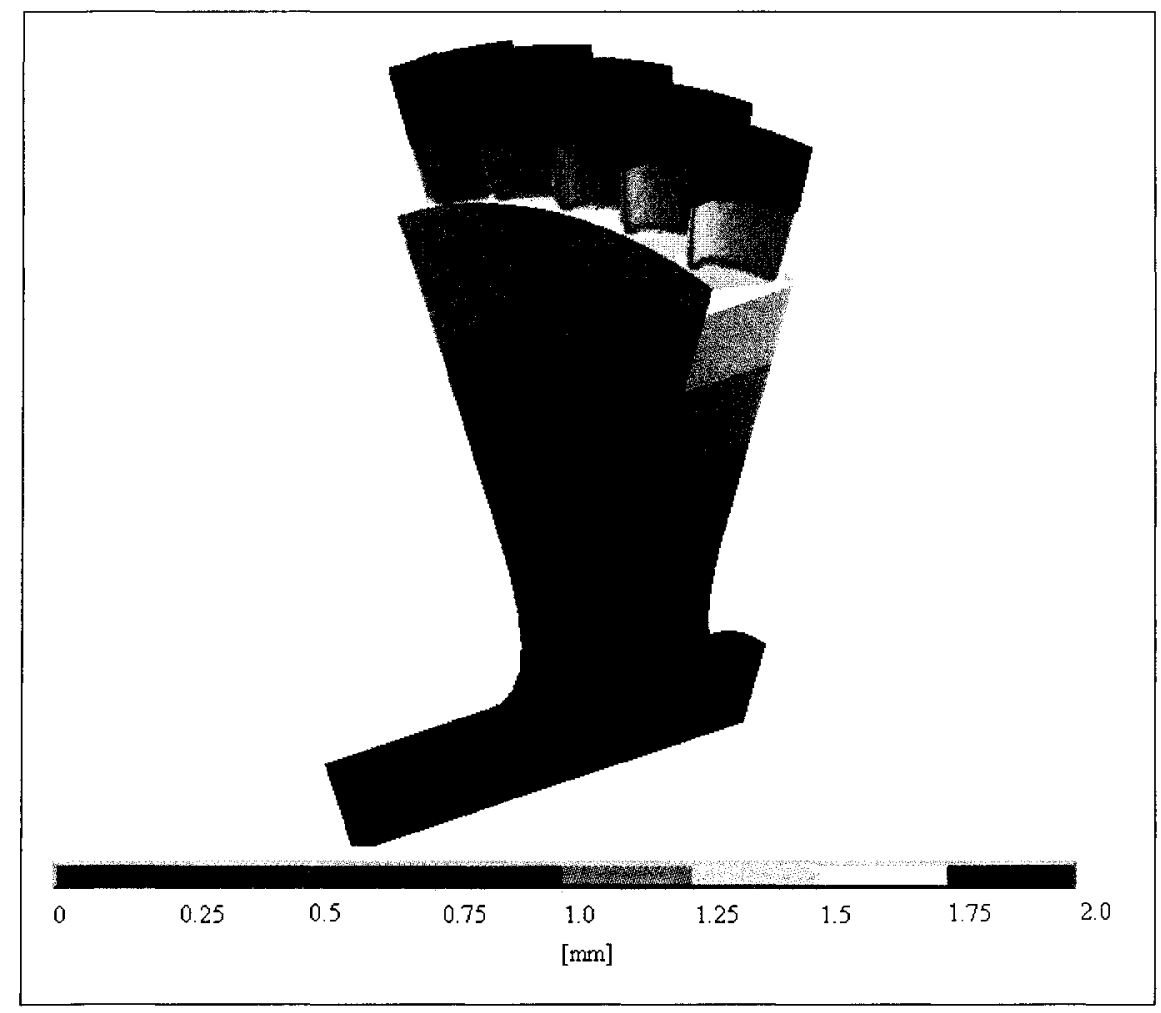

Figure 6.20: Radial growth of gas generator turbine due to thermal effects

To provide further validation for the design tool, the blade root stress must be calculated using ANSYS 9.0 and compared to the design tool output. For this analysis, a new model was created. The blade model and mesh are shown in Figure 6.21.

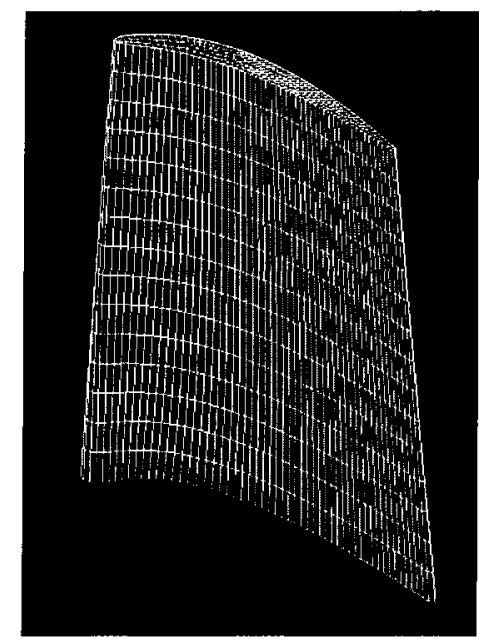

Figure 6.21: Gas generator turbine blade finite element mesh 
By constraining the hub portion of the blade shown in Figure 6.21, and rotating it at the prescribed RPM, the stress distribution may be calculated. The resulting stress distribution is illustrated in Figure 6.22.

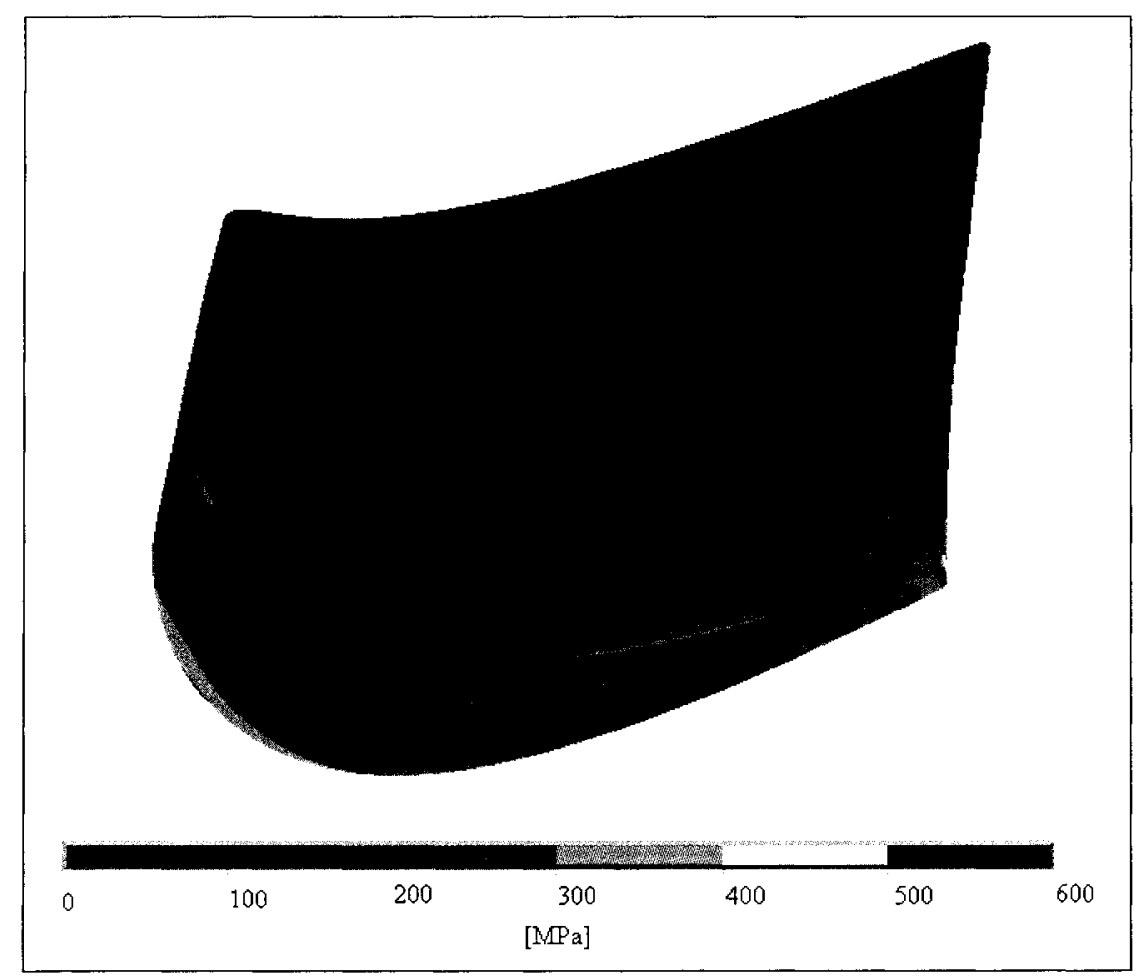

Figure 6.22: Blade root stress distribution (gas generator turbine)

As presented earlier, the maximum blade stress is located at the blade root trailing edge as a result of the thin trailing edges employed in the aerodynamic design. One may also note that the higher stress distributions exist surrounding the perimeter of the blade due to localized stress concentrations. Most importantly however, is the large green area where the stress level is of the order of $300 \mathrm{MPa}$. This result compares very closely to the value of $323 \mathrm{MPa}$ predicted by the design tool for the blade root stress.

Using the same blade model and mesh illustrated in Figure 6.21, a vibration analysis was conducted in ANSYS 9.0. Two analyses were conducted for the gas generator turbine design. A single vibration analysis was first conducted without the effects of centrifugal loading in order to determine the mode shapes and natural frequencies for a stationary rotor blade. A second vibration analysis was then conducted 
with the effects of centrifugal stiffening to determine the mode shapes at the intended design speed. The effects of centrifugal stiffening typically cause a slight increase in the modal frequencies at the design speed. The natural frequencies of the gas generator turbine rotor blades are illustrated in the Campbell diagram, shown in Figure 6.23.

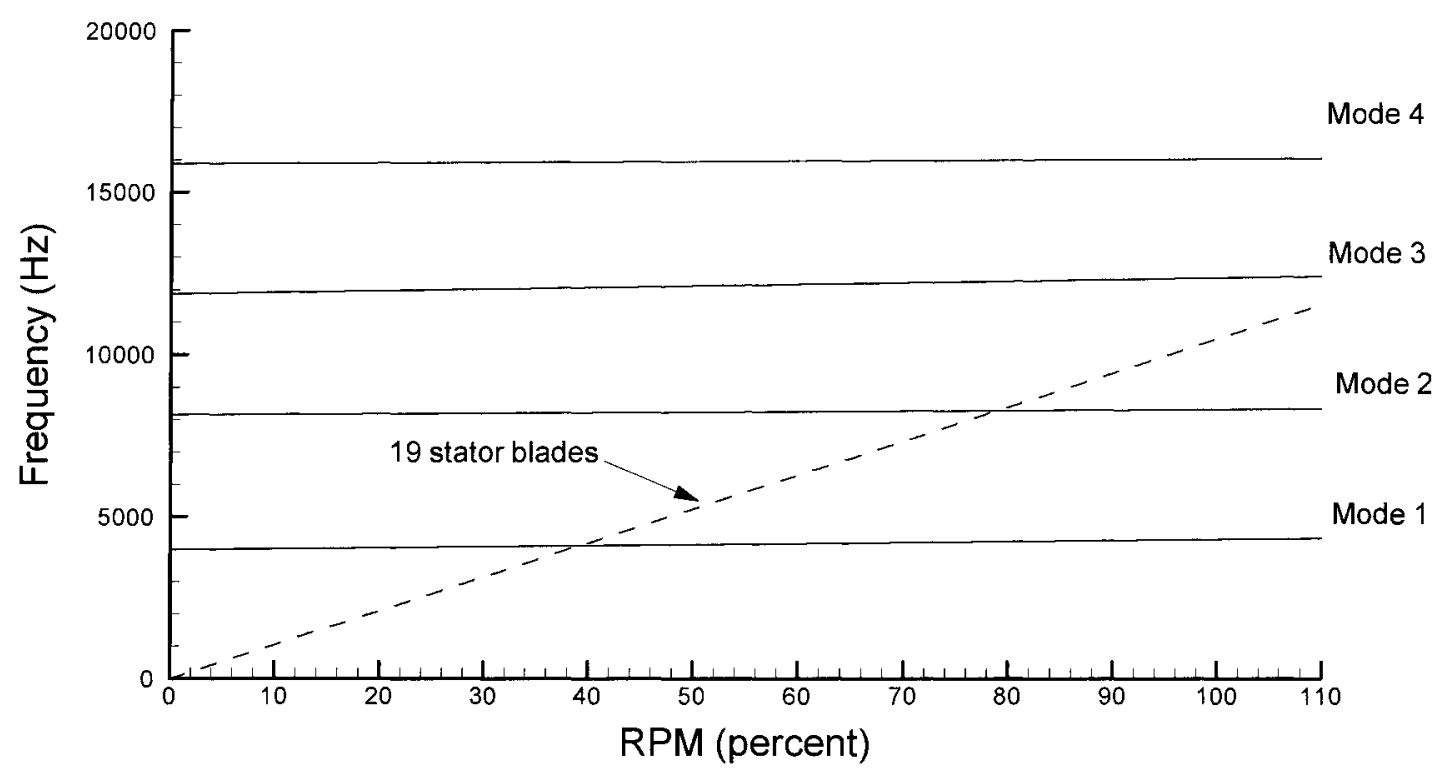

Figure 6.23: Gas generator turbine Campbell diagram

These results are used to ensure the avoidance of HCF in the turbine rotor blades. Based Figure 6.23, it is evident that Modes 2 and 3 will be avoided during normal design point operation. Unfortunately, at lower power demands, and thus lower RPM, there exists a possibility for excitation of the second mode. This phenomenon is related to the shedding of the stator wakes upstream of the turbine rotor. As a result of the rotating turbine blades, this causes oscillating pressure fluctuations to be experienced by the downstream rotor. It is up to the designer to ensure that, in all possible situations, the frequency of the oscillating pressure fluctuations does not match any of the natural frequencies of the turbine blade. Detailed analyses regarding blade vibration and aero elastic effects of the turbomachinery blading are being further investigated by another team member. 


\subsubsection{Structural Design Validation - Power Turbine}

This section will present the results of the ANSYS structural simulations conducted for the power turbine. Much of the discussion presented for the gas generator turbine will also apply to these results. A complete inertial rotor analysis will be presented first. This will be followed by the preliminary thermal and vibration analyses, as well as an estimation for the blade root stresses.

The computational mesh for the power turbine model is shown in Figure 6.24. Using this mesh, the inertial and thermal analyses were conducted. Once again, one will notice the large number of elements required to adequately resolve the geometry of the blade leading and trailing edges.

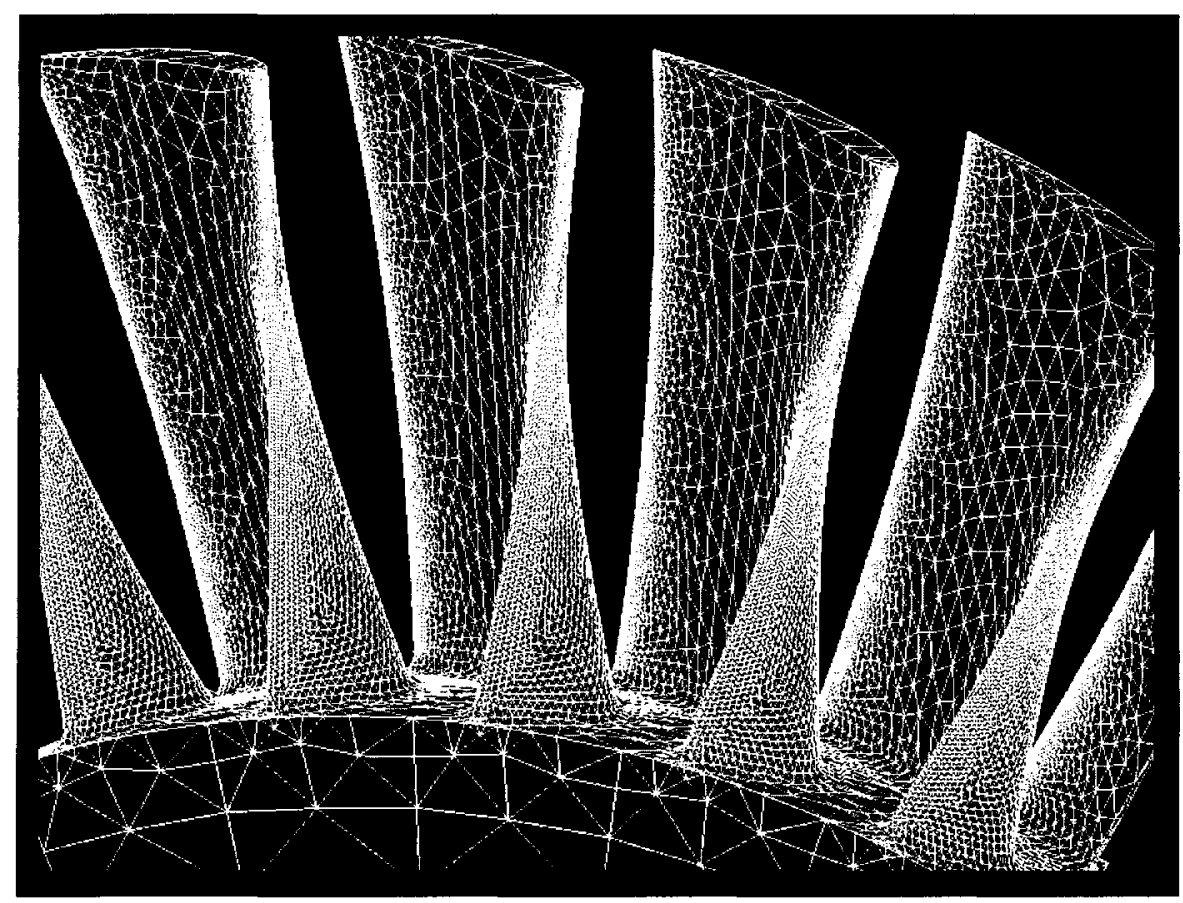

Figure 6.24: Power turbine finite element mesh

The results of the inertial analysis are presented in Figures 6.25 and 6.26. Figure 6.25 presents the von Mises stress distribution on the power turbine rotor. Due to the high aspect ratio of the power turbine blades, the stress distribution throughout the blade section is more complicated than the gas generator blade sections. This will require some 
additional attention in the detailed design of this component. Similar to the gas generator turbine rotor, the maximum stresses in this component occur at the blade trailing edges. Future work should consider enlargement of the trailing edges as well as the evaluation of its effects both structurally and aerodynamically. Typically, the largest stresses in a gas turbine blade are located at the blade hub. In Figure 6.25, the largest stresses occur throughout 50 percent of the blade span. For this reason, it is advised that the blade profiles be thickened in the lower half of the span.

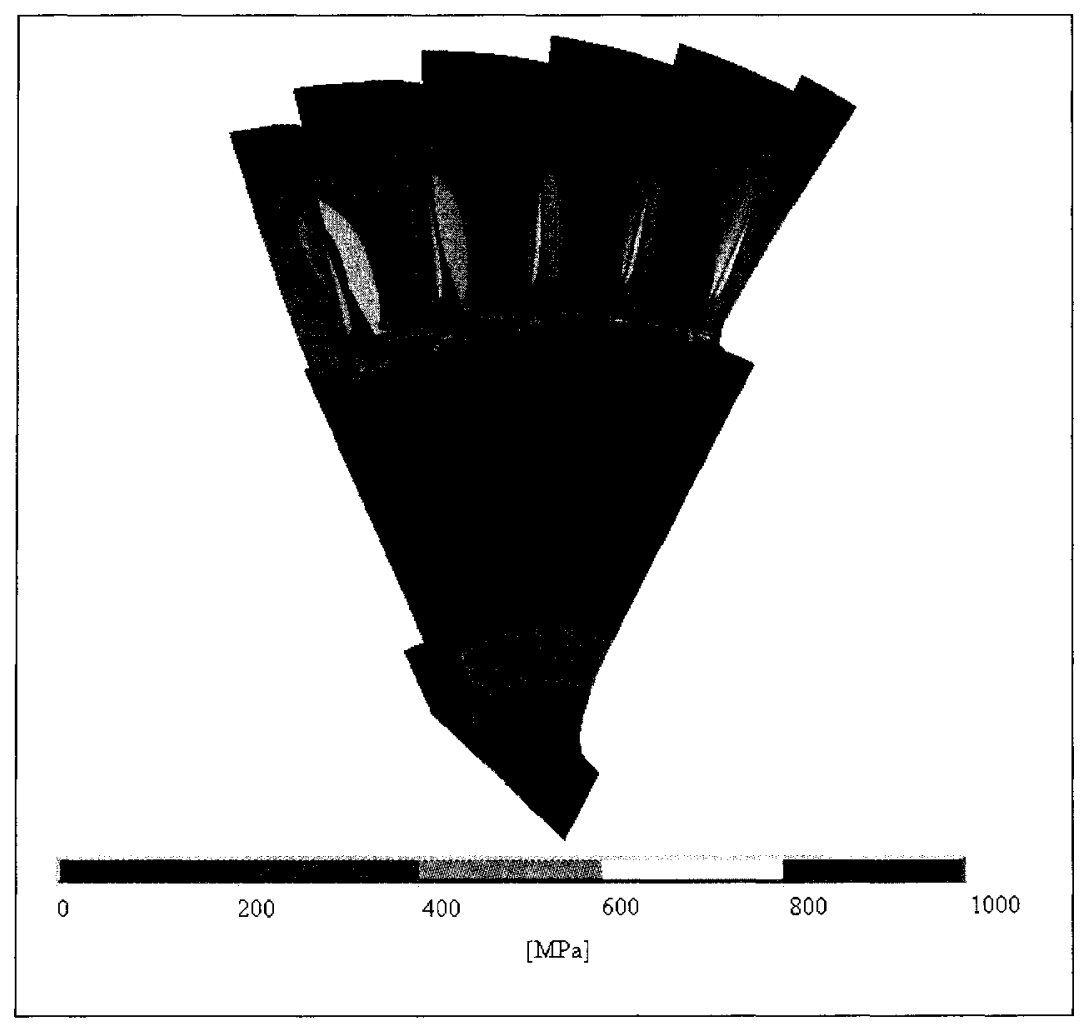

Figure 6.25: Rotor von Mises stress distribution (power turbine) 


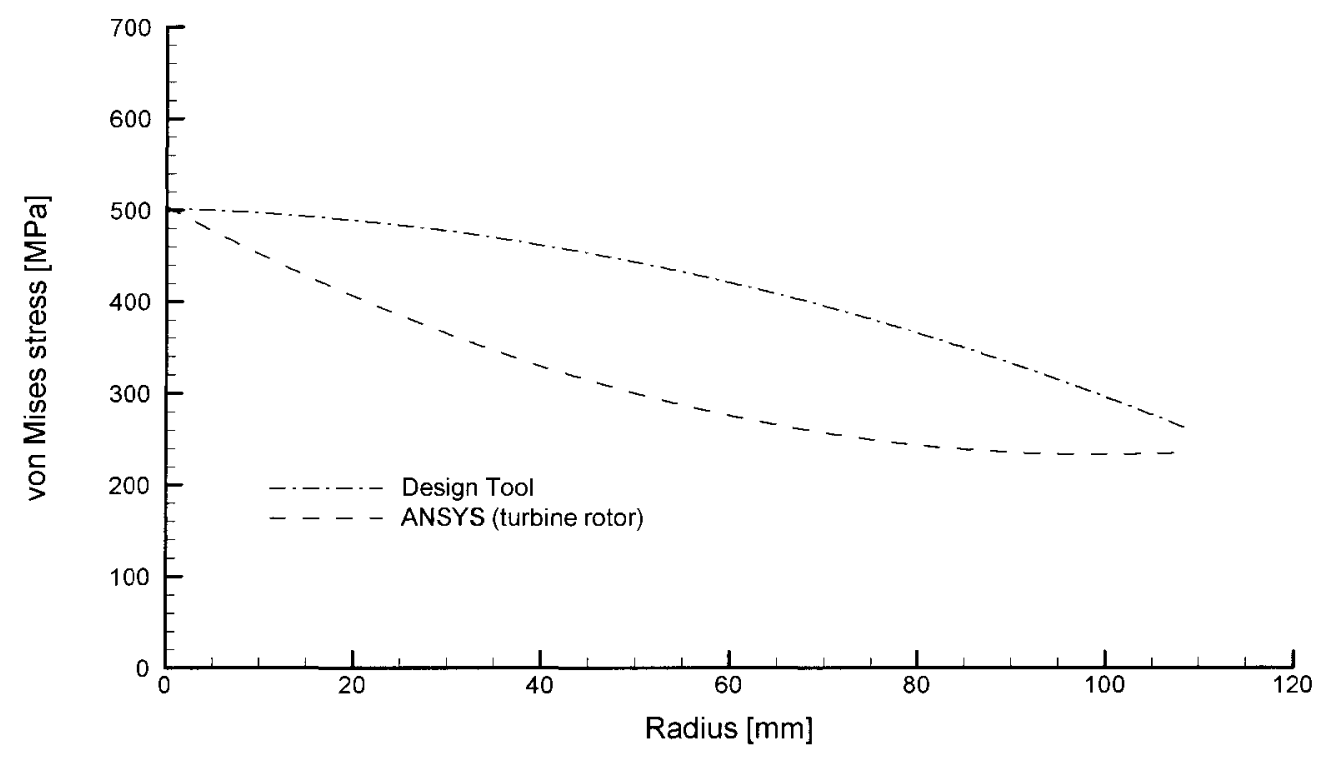

Figure 6.26: Rotor stress distribution - ANSYS vs design tool (power turbine)

Figure 6.26 illustrates the comparison of the design tool results with those obtained from the ANSYS solution. Once again, the results are quite close at the extremities of the graph; however, the trends in the results are opposite. As before, this is a result of the added disk thickness imposed by the modeling of the shaft and fillet in ANSYS 9.0. At a given radial distance, the ANSYS model is slightly larger in the thickness direction, thus reducing the magnitude of the von Mises stresses locally.

The assumed temperature distribution for the power turbine is shown in Figure 6.27. As with the gas generator simulations, these results do not account for convective heat transfer or disk cooling. From this prescribed temperature distribution, the rotor thermal growth was calculated. The results of this simulation are shown in Figure 6.28. A radial growth of approximately $2.2 \mathrm{~mm}$ (or about 4 percent) is predicted by the ANSYS simulation. The radial growth due to centrifugal effects alone is approximately $0.5 \mathrm{~mm}$. The combination of these effects total a radial growth of $2.7 \mathrm{~mm}$ at the design conditions. This must be accounted for in the casing design. 


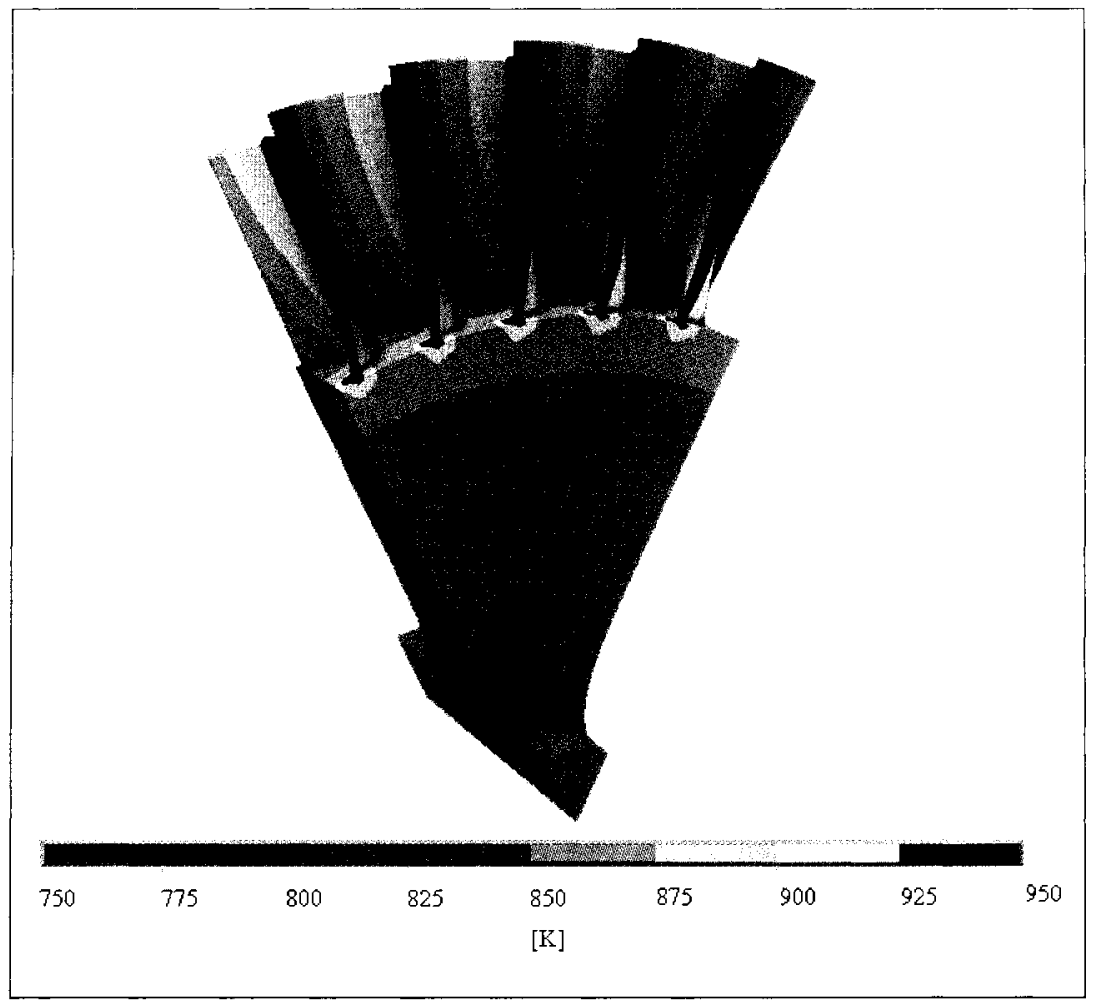

Figure 6.27: Power turbine rotor temperature distribution

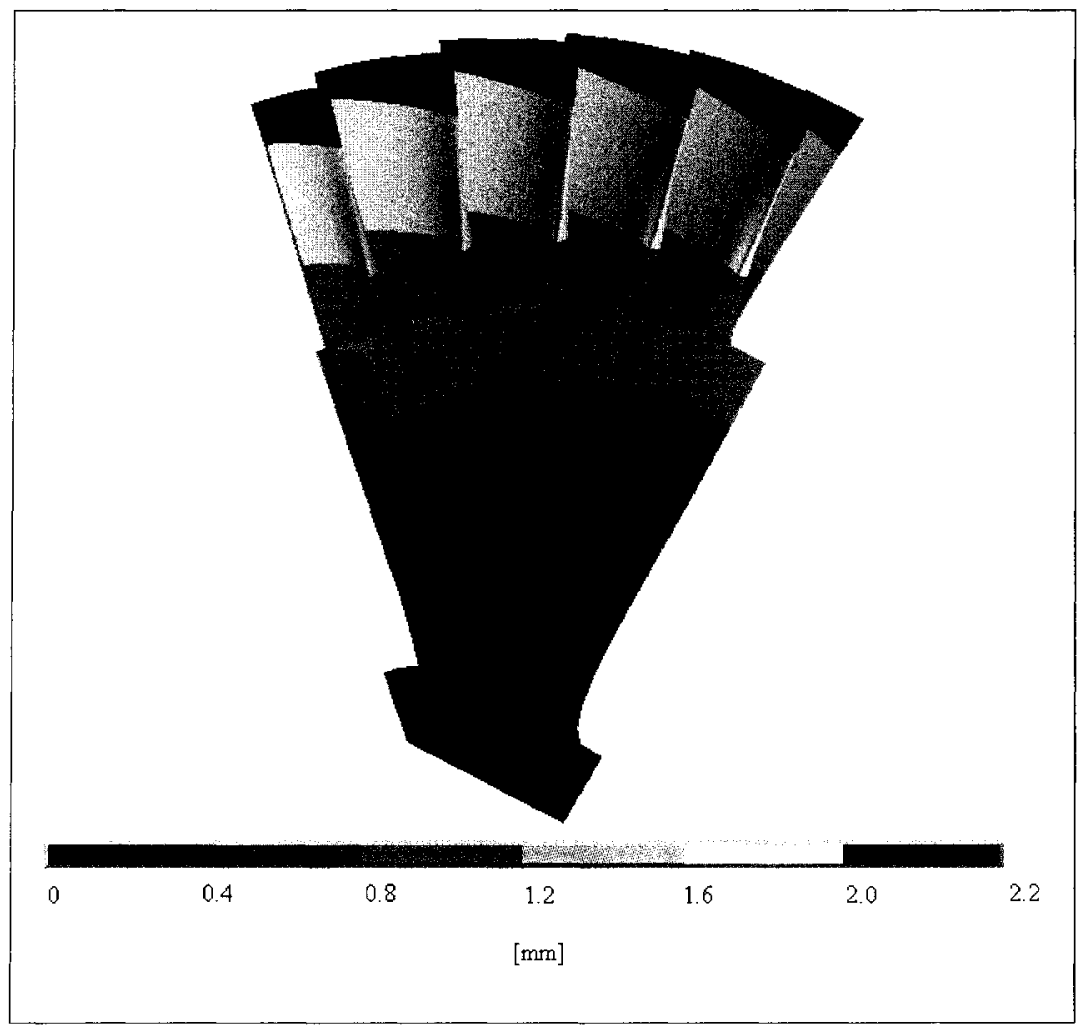

Figure 6.28: Radial growth of power turbine rotor due to thermal effects 
For the blade root stress and vibration analyses, a separate ANSYS model was analyzed. Using a single blade, a structured mesh was created as illustrated in Figure 6.29. The structured mesh greatly reduces the node count, particularly at the blade leading and trailing edges, where high aspect ratio elements provide the necessary curvature to accurately model the blade geometry.

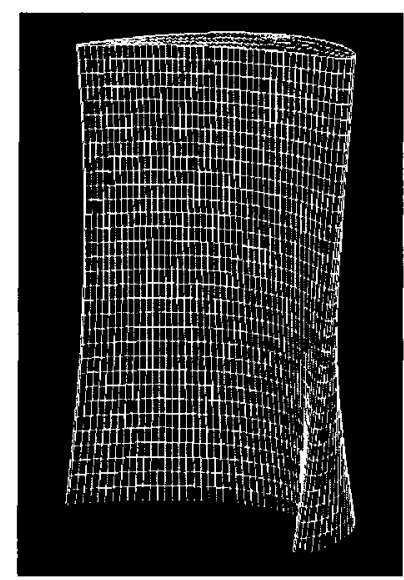

Figure 6.29: Power turbine blade finite element mesh

According to the results of the design tool, a blade root stress of approximately $400 \mathrm{MPa}$ is predicted. Figure 6.30 displays a contour plot of the radial stresses for the entire blade, emphasizing the root section. From these results, it can be seen that a majority of the blade root is experiencing stresses in the regime predicted by the design tool. There is a large section however, that is well under $200 \mathrm{MPa}$. This could be a result of the centrifugal bending occurring due to the three-dimensional stacking of the blade profiles. Further investigation is required. 


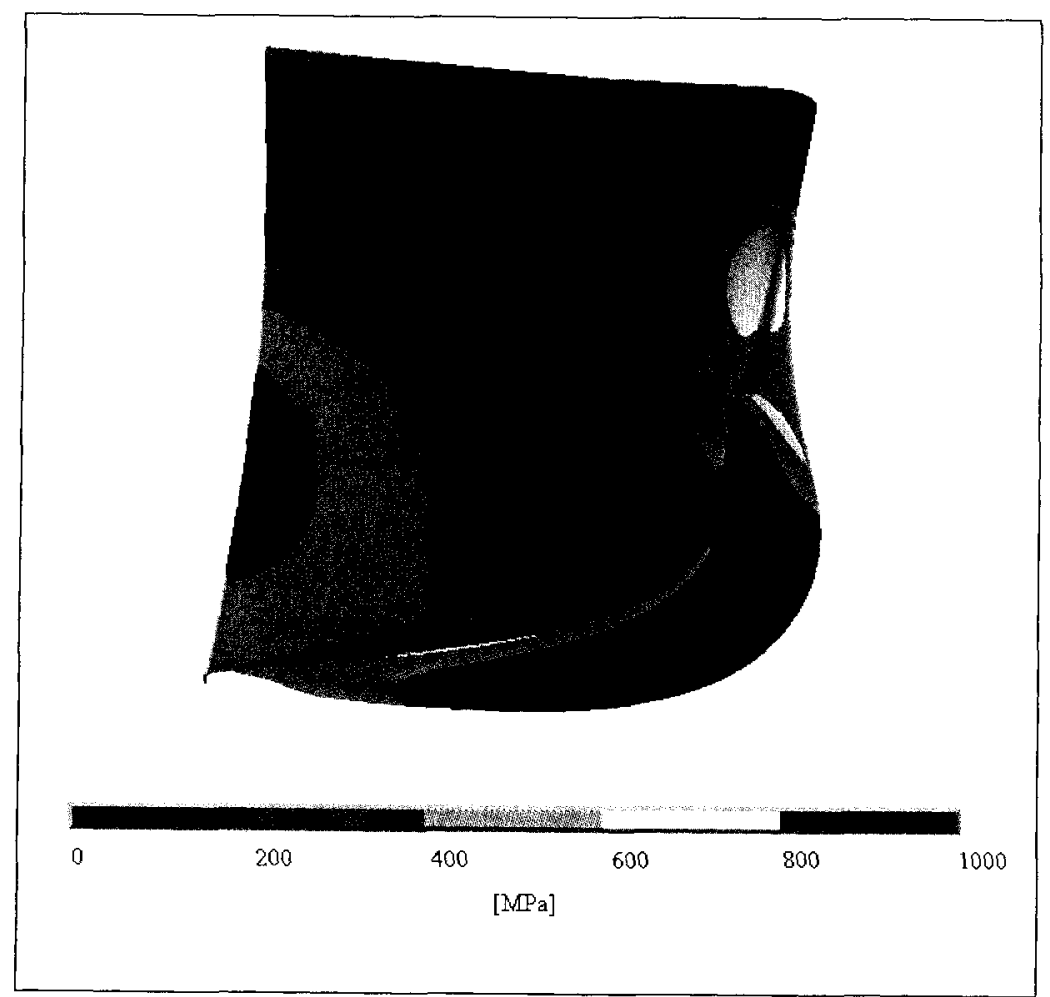

Figure 6.30: Blade root stress distribution (power turbine)

Finally, the results of the vibration analysis for the power turbine assembly are presented. The Campbell diagram indicating the first five modes and excitation frequency is displayed in Figure 6.31.

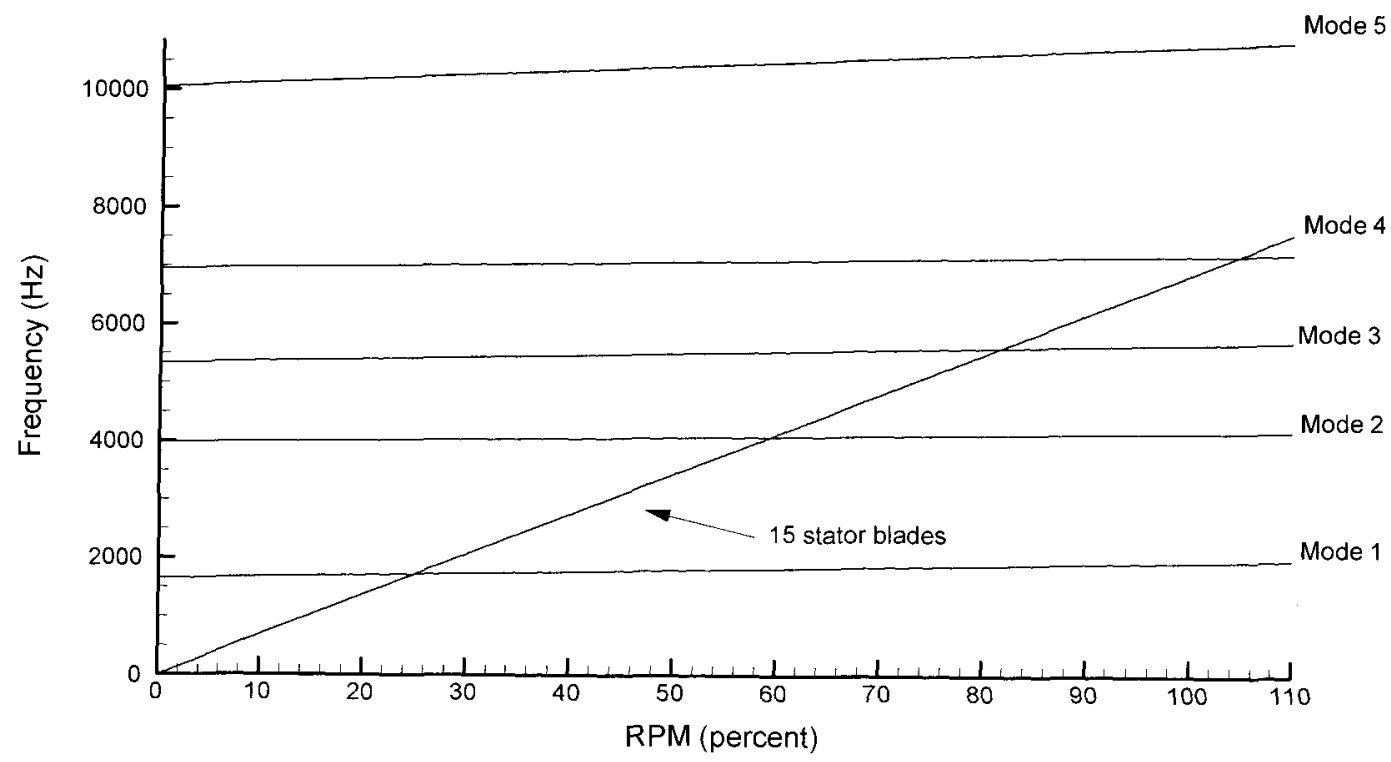

Figure 6.31: Campbell diagram for the power turbine assembly 
Based on the results of the current design, the excitation frequency at the design point is much too close to the fourth natural frequency of the turbine rotor blade. Due to the large frequency range predicted between the fourth and fifth mode, it would be advisable to attempt to increase the excitation frequency of the assembly. By increasing the number of stator blades to 19 instead of the current 15 , the forcing frequency could be raised to approximately $9000 \mathrm{~Hz}$, thus eliminating the concerns of $\mathrm{HCF}$ in the power turbine assembly.

This concludes the validation of the design tool both aerodynamically and structurally. Additional results have also been presented to provide further confidence in the design of the two turbine stages. The recommend future work and conclusions are presented next. 


\section{Chapter 7}

\section{Conclusions and Recommendations}

This final chapter will present the reader with an overview of the accomplishments in the preliminary design of two axial flow turbine stages. It will provide a summary of the overall design, as well as its current status. A major component of the thesis was the development and validation of the comprehensive design tool for axial turbine design. The design tool was successfully validated and provides an excellent platform for the detailed design.

A number of recommendations are also presented. These are based on further actions required in the advancement of the preliminary design tool, as well as the next steps required to achieve a working engine prototype. 


\subsection{Conclusions}

The complete preliminary design of two gas turbine stages for a 1-MW gas turbine engine has been presented. A preliminary design tool was created to assist in the aerodynamic and structural design. From the initial concept and performance requirements, the turbomachinery configuration was selected. Included in the tool was the ability to produce initial estimates of the overall stage performance and stress distributions. To validate the outputs from the design tool, the geometry was modeled in CFX-BladeGen. These blade models were then used for more advanced CFD analysis using CFX-5. Solid models were also generated using Pro/ENGINEER Wildfire, and the stress distributions were evaluated using ANSYS 9.0.

It was found that the results of comprehensive design tool were comparable to the results obtained from CFD and FEA simulations. This proved the validity and accuracy of the design tool. Additional thermal and vibration analyses were also conducted in ANSYS 9.0 to gain further confidence in the design. These results have also been presented. A solid model of the complete turbine assembly was also created and is shown in Figure 7.1. This is the current status of the turbine components. With the preliminary design complete, the geometry may now be subjected to more detailed design and optimization. 


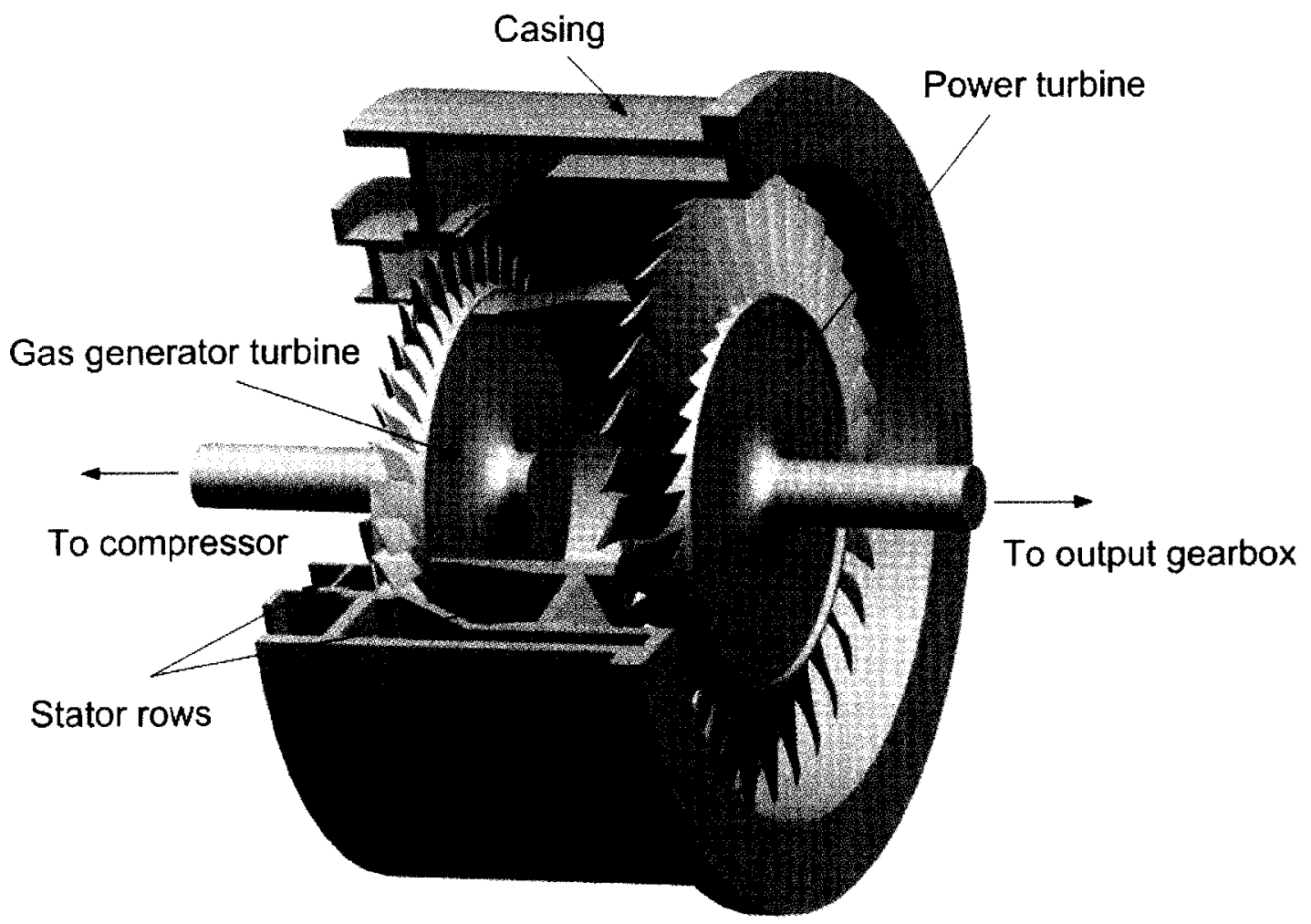

Figure 7.1: Turbine assembly 


\subsection{Recommendations}

With the preliminary aerodynamic and structural design completed, it is the responsibility of future team member(s) to concentrate on the detailed design of the turbine components. In addition to the detailed design, some advancement can be made to the preliminary design tool. Recommended actions include:

- Additional options for forced-vortex design. Currently, only free vortex design is available. By incorporating a variable forced-vortex design, the designer is able to design blade rows with a constant outlet angle or mass flow per unit area.

- A method to ensure constant (or nearly constant) blade tip radii. When designing blade rows of significant pressure ratio, a rather large annular flare is required to obtain a constant axial velocity. Additional options should be incorporated allow the user to specify whether a constant axial velocity or a constant blade tip radii are desired

- The addition of new incidence correlations. The current incidence calculations used in the design tool did not prove to be reliable as large over-accelerations were still present around the leading edge in the CFD analysis.

- An alternate method for determining the pressure loss coefficient due to tip clearances. The current method simply applies a correction factor to the resulting isentropic efficiency calculated for zero tip clearance. By adjusting the value for the tip clearance pressure loss coefficient, the user is able to assume a total pressure loss coefficient which yields the same isentropic stage efficiency calculated by the correction factor.

- Automating the entire design tool.

- The addition of a material database.

- The addition of heat transfer and thermal growth. Currently no indication of thermal growth is provided by the design tool. The results which have been presented have been obtained solely from ANSYS.

- A more advanced blade attachment sizing. The current blade attachment design provides only a staring point for preliminary design purposes. Significant 
detailed design is still required for this component if it is expected to be used in the actual engine.

- Current trends in multi-disciplinary optimization make this design tool very useful as part of a future optimization project. The tool could be run as an object function within a larger optimization scheme in order to conduct trade-offs between structural and aerodynamic design considerations.

Additional recommendations for the overall turbine design are also provided. These recommendations do not directly relate to the preliminary design tool, but are required to further the development of the engine components. These recommendations include:

- Advanced conjugate heat transfer analysis.

- Disk cooling and air systems.

- Further development relating to mechanical design and manufacturing methods.

- A casing heat transfer analysis.

- Rotor/shaft attachment mechanisms.

- Prototype manufacture and testing. 


\section{References}

Ainley, D.G., and Mathieson, G.C.R., A method of performance estimation for axial flow turbines, Aeronautical Research Council, London, ARC R and M No. 2974, 1951.

Anonymous, $C F X-5.7 .1$ user manual, 2004

Arakere, N.K., and Moroso, J., Fatigue failure in high-temperature single crystal superalloy turbine blades, High Temperature Materials and Processes, Vol. 20, No. 2, 2001

Ashby, M., A first report on deformation mechanism maps, Acta Met., Vol. 20, pp. 887997, July 1972

Balje, O.E., Turbomachines: A guide to design, selection, and theory, John Wiley \& Sons Inc. 1981

Byerley, A.R., Boyer, K.M., and Halliwell, I., Using design envelopes to aid in the preliminary design of rotating turbomachinery, Proceedings of ASME TURBO EXPO 2004, June 14-17 2004

Cairo, R.R., and Sargent, K.A., A scientific approach to the process development bonded attachments for high speed rotor application, Journal of Engineering for Gas Turbines and Power, Vol. 124, pp. 190-195, January 2002

Chen, H., and Baines, N.C., The aerodynamic loading of radial and mixed flow turbines, Int Journ Mech Sci., Vol. 36, pp. 63-79, 1994

Claudio, R.A., Branco, C.M., Gomes, E.C., Byrne, J., Harrison, G.F., and Winstone, M.R., Fatigue life prediction and failure analysis of a gas turbine disk using the finite element method, Fatigue Fract Engng Mater Struct, Vol. 27, pp. 849-860, 2004

Coffin, L.F., and Schenectady, N.Y., A study of the effects of cyclic thermal stresses on a ductile metal, Transactions of the ASME, Vol. 76, pp. 931-961, 1954

Corriveau, D., and Sjolander, S.A., Influence of loading distribution on the performance of transonic high pressure turbine blades, ASME Journal of Turbomachinery, Vol. 126, pp.288-296, April 2004

Craig, H.R.M., and Cox, H.J.A., Performance estimation of axial flow turbines, Heat and Fluid Flow, Vol.1, No. 2, 1971

Dawes, W.N., A comparison of zero and one equation turbulence models for turbomachinery calculations, ASME Paper No. 90-GT-303, 1990. 
Demeulenaere, A., Leonard, O., and Van den Braembussche, R., A two-dimensional Navier-Stokes inverse solver for compressor and turbine blade design, Proceedings of the Institute of Mechanical Engineers, Vol. 211, Part A, 1997

Denton, J.D., Loss Mechanisms in Turbomachinery, ASME Journal of Turbomachinery, Vol. 115, pp.621-656, October 1993

Dunavant, J.C., and Erwin, J.R., Investigation of a related series of turbine-blade profiles in cascade, Technical note TN 3802, NACA, Washington DC, 1956

Dunham, J., and Came, P.M., Improvements to the Ainley/Mathieson method of turbine performance prediction, ASME Journal of Engineering for Power, pp. 252-256, July 1970

Fessler, H., and Thorpe, T.E., Centrifugal stresses in rotationally symmetrical gas turbine disks, Journal of Strain Analysis, Vol. 3, No. 2, pp.135-141, 1968

Fielding, L., Handheld calculator programs for rotating equipment design, McGraw-Hill, Inc., 1983

Figge, E.E., An empirical equation relating fatigue limit and mean stress, NASA TN D3883,1967

Filsinger, D., Szwedowicz, J., and Schafer, O., Approach to unidirectional coupled CFDFEM analysis of axial turbocharger turbine blades, ASME Journal of Turbomachinery, Vol. 124, pp. 125-131, January 2002

Gallus, H.E., Zeschky, J., and Hah, C., Endwall and unsteady flow phenomena in an axial turbine stage, ASME Paper No. 94-GT-143, 1994

Gauthier, J.E.D, Gas turbine course, Supplementary Course Notes, Department of Mechanical and Aerospace Engineering, Carleton University, July 2005

Graham, J.A.H., Investigation of tip clearance cascade in a water analogy rig. ASME Journal of Engineering for Gas Turbines and Power, Vol. 108, pp. 38-46, 1986

Hagg, A.C., Sankey, G.O., The containment of disk burst fragments by cylindrical shells, Paper No. ASME 73PWR-2, 1973

Haigh, R.E., and Murdoch, M.L., Analysis of centrifugal stresses in turbine wheels, Journal Mechanical Engineering Science, Vol. 5, No. 1, pp. 66-74, 1963

Haller, B.R., Full 3D turbine blade design, VKI Lecture Series on "Secondary and Tip Clearance Flows in Axial Turbines" the von Karman Institute for Fluid Dynamics, February 1997 
Haller, B., and Anderson, J., Development of new high load/high lift transonic shrouded HP gas turbine stage design - A new approach for turbomachinery, Proceedings of ASME TURBO EXPO 2002, June 3-6 2002

Heck, K.A., Smith, J.S., and Smith, R., INCONEL Alloy 783: An oxidation-resistant, low expansion superalloy for gas turbine applications, ASME Journal of Engineering for Gas Turbines and Power, Vol. 120, pp. 363-367, April 1998

Ho, Y.H., and Lakshminarayana, B., Computational modeling of three-dimensional endwall flow through a turbine rotor cascade with strong secondary flows, ASME Journal of Turbomachinery, Vol. 118, pp.250-261, April 1996

Huda, Z., Development of design principles for a creep-limited alloy for turbine blades, Journal of Materials Engineering and Performance, Vol. 4, No. 1, February 1995

Hummel, F., Lotzerich, M., Cardamone, P., and Fottner, I.L., Surface roughness effects on turbine blade aerodynamics, Proceedings of ASME TURBO EXPO 2004, June 14-17 2004

Japikse, D., and Baines, N.C., Introduction to turbomachinery, Oxford University Press, 1997

Kacker, S.C., and Okapuu, U., A meanline prediction method for axial flow turbine efficiency, ASME Journal of Engineering for Power, Vol. 104, pp. 111-119. January 1982

Kim, J.H., Kim, K.H., Joung, K.K., Ham, K.C., Song, J.I., and Bae, S.I., Fatigue analysis of vane components for gas turbine engine, Key Engineering Materials, Vols. 183-187, pp. 1029-1034, 2000

Larson, F.R., and Miller, J., A time-temperature relationship for rupture and creep stresses, Transactions of the ASME, 1952

Logan, E., Handbook of turbomachinery, Marcel Dekker Inc., 2003

Luo, J., and Lakshminarayan, B., Navier-Stokes analysis of turbine flowfield and external heat transfer, Journal of Propulsion and Power, Vol. 11, No. 2, pp. 221-229, 1995

Mangonon, P.L., The principles of materials selection for engineering design, PrenticeHall, Inc., 1999

Manson, S.S., Interfaces between fatigue, creep and fracture, International Journal of Fracture Mechanics, Vol. 2, pp.337-363, 1966

Manson, S.S., and Haferd, A.M., A linear time relationship for extrapolation of creep and stress rupture data, NACA TN 2890, 1953 
Meguid, S.A., Kanth, P.S., and Czekanski, A., Finite element analysis of fir-tree region in turbine disks, Finite Elements in Analysis and Design, Vol. 35, pp. 305-37, 2000

Menter, F.R., Two equation eddy-viscosity turbulence models for engineering application, AIAA Journal, Vol. 32, No. 8, 1994

Moustapha, H., Zelesky, M.F., Baines, N.C., and Japikse, D., Axial and radial turbines, Concepts ETI Inc., 2003

Nichol, K.L., Assessment of current turbine engine high cycle fatigue test methods, ASME Journal of Engineering for Gas Turbines and Power, Vol. 125, pp.760-765, 2003

Ostergren, W., Correlating of hold time effects of elevated temperature modified damage function, 1978 ASME-MPC symposium on creep fatigue interaction, MPC-3, ASME, 1976

Rao, J.S., Turbomachine blade vibration, John Wiley and Sons, Ltd., 1991

Rao, M.N., Processing and evaluation of creep and fatigue resistant alloys for gas turbine applications, Tran. Indian Inst. Met., Vol. 49, No. 4, August 1996

Radhakrishna, C., and Rao, K.R., Studies on creep/stress rupture behaviour of superalloy 718 weldments used in gas turbine applications, Materials at High Temperatures, Vol. 12, No. 4, 1994

Ristic, D., Lakshminarayan, B., and Chu, S., Three-dimensional flowfield downstream of an axial flow turbine rotor, Journal of Propulsion and Power, Vol. 15, No. 2, 1999

Roark, R.J., and Young, W.C., Formulas for stress and strain, McGraw-Hill, 1975

Roelke, R.J., and Haas, J.E., The effect of rotor blade thickness and surface finish on the performance of a small axial flow turbine, Journal of Engineering for Power, Vol. 105, pp.377-382, April 1983

Salam, I., Tauqir, A., and Khan, A.Q., Creep-fatigue failure of an aero engine turbine blade, Engineering Failure Analysis, Vol. 9, pp.335-347, 2002

Saravanamuttoo, H.I.H., Rogers, G.F.C., and Cohen, H., Gas turbine theory, $5^{\text {th }}$ edition, Pearson Education Limited, 2001

Saywer, J.W., Sawyer's gas turbine engineering handbook, Volume I: Theory and Design, Turbomachinery International Publications, 1985

Shaw, H., An improved blade root design for axial flow compressors (and turbines), The Aeronautical Journal of the Royal Aeronautical Society, Vol. 74, pp. 589-592, July 1970 
Shiratori, E., Hoshiya, M., and Sakurai, N., Elastic design of fir-tree joints for blade fastenings, Bulletin of the Tokyo Institute of Technology, No. 80, pp.25-73, 1967

Sieverding, C. H., Axial turbine performance prediction methods, Thermodynamics and Fluid Mechanics of Turbomachinery, Vol. 2, pp.737-784, 1985a

Sieverding, C.H., Recent progress in the understanding of basic aspects of secondary flows in turbine blade passages, ASME Journal of Engineering for Gas Turbines and Power, Vol. 107, pp. 248-258, April 1985b

Sims, C.T., Stoloff, N.S., and Hagel, W.C., Superalloys II, John Wiley \& Sons, Inc., 1987

Sjolander, S.A., Overview of tip-clearance effects in axial turbines, VKI Lecture Series on "Secondary and Tip Clearance Flows in Axial Turbines" the von Karman Institute for Fluid Dynamics, 1997

Sjolander, S.A., Fluid machinery/turbomachinery, Supplementary Course Notes, Department of Mechanical and Aerospace Engineering, Carleton University, December 2002

Sjolander, S.A., and Amrud, K.K., Effects of tip clearance on blade loading in a planar cascade of turbine blades, ASME Journal of Turbomachinery, Vol. 109, pp. 237-244, 1987

Smith, S.F., A simple correlation of turbine efficiency, The Aeronautical Journal of the Royal Aeronautical Society, Vol. 69, pp. 467, 1965

Subhas, B.K, Balakrishna, H.K., Ramachandra, K., and Bhat, R., Dimensional instability studies in machining of Inconel 718 nickel based superalloy as applied to aerogas turbine components, Journal of Engineering for Gas Turbines and Power, Vol. 122, pp.55-61, 2000.

Subramani, D.A., Ramamurti, V., and Sridhara, K., Finite element analysis of an automotive turbocharger turbine wheel, 1995 Design Engineering Technical Conferences, Vol. 3, Part B, ASME 1995

Tong, M.T., Halliwell, I., and Ghosn, L.J., A computer code for gas turbine engine weight and disk life estimation, Journal of Engineering for Gas Turbines and Power, Vol. 126, pp. 265-270, 2004

Vazquez, R., Cadrecha, D., and Torre, D., High stage loading low pressure turbines. A new proposal for an efficiency chart, Proceedings of ASME TURBO EXPO 2003, June 2003 
Virr, G.P., Chew, J.W., and Coupland, J., Application of computational fluid dynamics to turbine disk cavities, ASME Journal of Turbomachinery, Vol. 116, pp. 701-708, October 1994

Xiao, X., McCarter, A.A., and Lakshminarayana, B., Tip clearance effects in a turbine rotor: Part I-Pressure field and loss, ASME Journal of Turbomachinery, Vol. 123, pp. 296-304, April 2001a

Xiao, X., McCarter, A.A., and Lakshminarayana, B., Tip clearance effects in a turbine rotor: Part II -Velocity field and flow physics, ASME Journal of Turbomachinery, Vol.123, pp.305-313, April 2001b

Watanabe, H., Okamoto, H, Guo, S., Goto, A., and Zangeneh, M., Optimization of microturbine aerodynamics using CFD, inverse design and FEM structural analysis $\left(2^{\text {nd }}\right.$ report: turbine design), Proceedings of ASME TURBO EXPO 2004, GT2004-53583, June 14-17 2004

Wilson, D.G., and Korakianitis, T., The design of high-efficiency turbomachinery and gas turbines, $2^{\text {nd }}$ Edition, Prentice Hall 1998

Yaras, M.I., and Sjolander, S.A., Effects of simulated rotation on tip leakage in a planar cascade of turbine blades: Part I - Tip gap flow, ASME Journal of Turbomachinery, Vol. 114 , pp. 652-659, July 1992

Zhu, J., and Sjolander, S.A., Improved profile loss and deviation correlations for axialturbine blade rows, Proceedings of ASME TURBO EXPO 2005, GT2005-69077, June 692005

Zweifel, O., The spacing of turbomachine blading especially with large angular deflection, The Brown Boveri Review, pp. 436-444, December 1945 


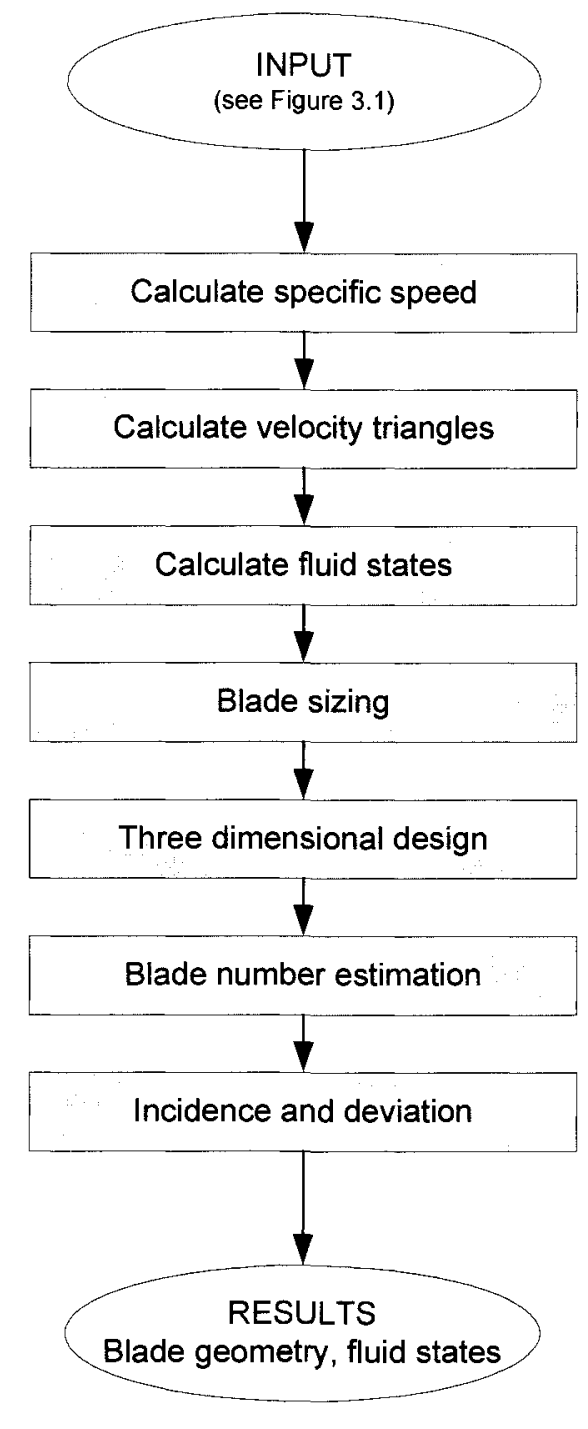

Figure A.1: Aerodynamic design flow chart 


\section{Appendix A2 - Performance Prediction Flow Chart}

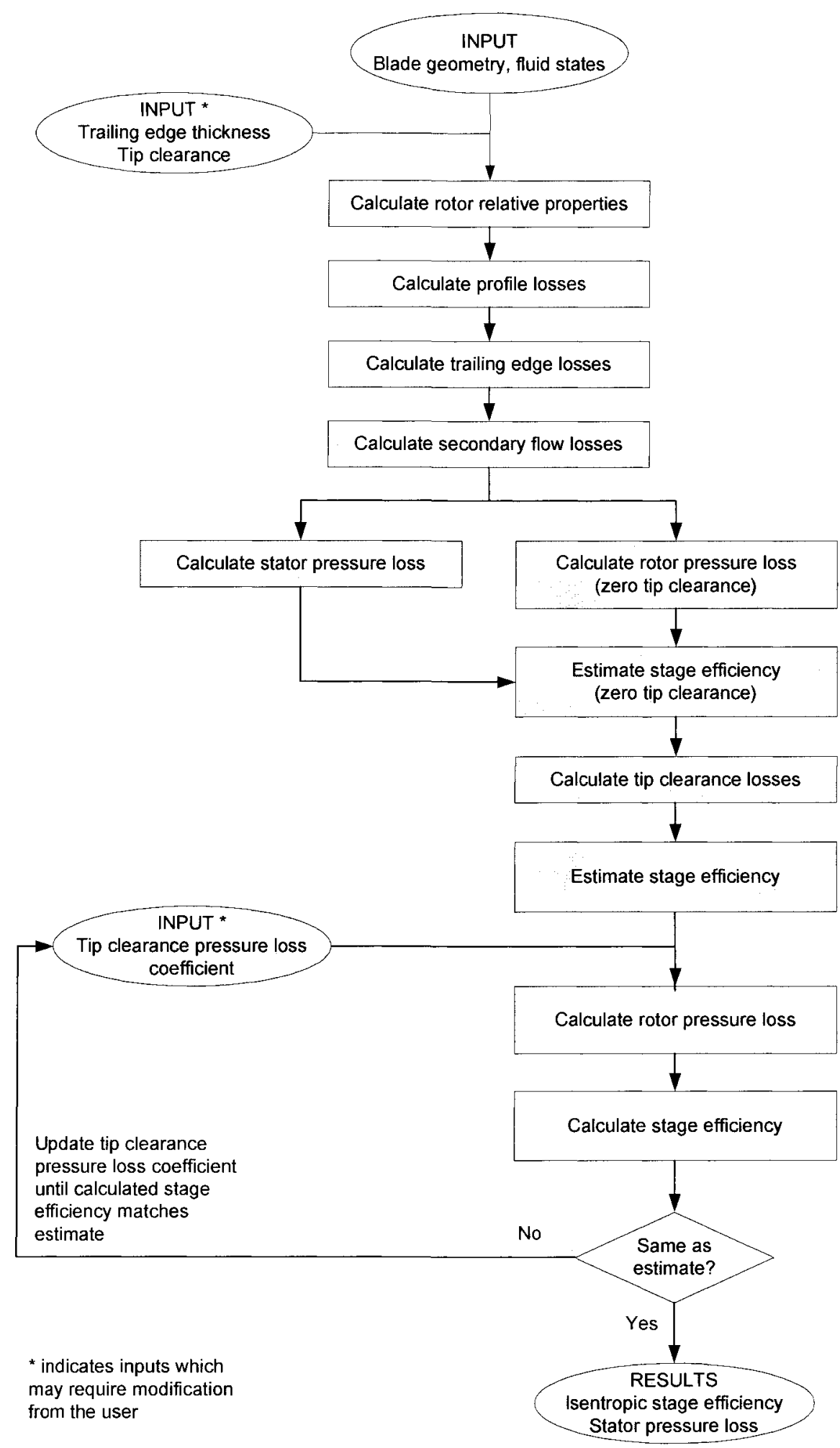

Figure A.2: Performance prediction flow chart 


\section{Appendix B1 - Design Tool Inputs}

Design Parameters:

\begin{tabular}{|c|c|c|}
\cline { 3 - 3 } \multicolumn{2}{c|}{} & Typical Values \\
\hline$\psi$ & Stage loading coefficient & $1.0-3.0$ \\
\hline$\Lambda$ & Flow coefficient & $0.4-1.2$ \\
\hline $\mathrm{C}_{\mathrm{D}}$ & Degree of reaction & $0-1.0$ \\
\hline $\mathrm{C}_{\mathrm{L}}$ & Blockage factor & $0.9-1.0$ \\
\hline $\mathrm{AR}$ & Zwiefel lift coefficienct & $0.7-1.3$ \\
\hline$\alpha_{1}$ & Aspect ratio & $0.5-3.0$ \\
\hline $\mathrm{N}$ & Inlet swirl angle [deg] & $0-70$ \\
\hline
\end{tabular}

\begin{tabular}{|c|c|c|}
\hline TC & Tip clearance [\%] & $0.4-2.0$ \\
\hline TET & Trailing edge thickness $[\mathrm{mm}]$ & $0.3-1.0$ \\
\hline
\end{tabular}

\begin{tabular}{|c|c|c|}
\hline$\zeta$ & Blade attachment angle [deg] & $0-70$ \\
\hline$\varepsilon_{\mathrm{c}}$ & Casing compressive strain & $0.05-0.10$ \\
\hline
\end{tabular}

Table B.1: Design parameter inputs

* The rotational speed will vary depending on the design application. Axial turbine limits are usually imposed by maximum permissible values of $K A N^{2}$. These have been presented in text.

Material Properties:

\begin{tabular}{|c|c|}
\hline$\rho$ & Density (rotor, casing) \\
\hline$\sigma_{\text {UTS }}$ & Ultimate tensile strength (rotor, casing) \\
\hline$\sigma_{\mathrm{Y}}$ & Yield strength (rotor, casing) \\
\hline LM & Larsen-Miller parameter (rotor) \\
\hline $\mathrm{E}$ & Young's modulus (casing) \\
\hline$\varepsilon$ & Elongation to fracture (casing) \\
\hline$v$ & Poisson's ratio \\
\hline
\end{tabular}

Table B.2: Material property inputs 
Fluid Properties:

\begin{tabular}{|c|c|}
\hline $\mathrm{R}_{\text {air }}$ & Gas constant $[\mathrm{J} / \mathrm{kgK}]$ \\
\hline $\mathrm{C}_{\mathrm{p}}$ & Specific heat at constant pressure $[\mathrm{J} / \mathrm{kgK}]$ \\
\hline$\gamma$ & Ratio of specific heats \\
\hline$\mu$ & Fluid viscocity \\
\hline
\end{tabular}

Table B.3: Fluid property inputs

Design Specifications:

\begin{tabular}{|c|c|}
\hline $\mathrm{T}_{01}$ & Turbine inlet temperature \\
\hline $\mathrm{P}_{01}$ & Turbine inlet pressure \\
\hline$\Delta \mathrm{h}_{0}$ & Specific work output \\
\hline $\mathrm{m}_{\text {air }}$ & Mass flow rate \\
\hline
\end{tabular}

\begin{tabular}{|c|c|}
\hline$T_{03 \mathrm{~d}}$ & Turbine exit pressure by design * \\
\hline $\mathrm{P}_{03 \mathrm{~d}}$ & Turbine exit temperature by design * \\
\hline$\eta$ & Total-to-total isentropic efficiency * \\
\hline
\end{tabular}

Table B.4: Design specification inputs

* These specifications have been provided by the overall engine performance specifications. They are used for specific speed calculations and initializing the performance prediction algorithm. 


\section{Appendix B2 - Design Tool Outputs}

In addition to the fluid state and geometrical outputs summarized in Tables 3.2 and 3.3 of Chapter 3, a number of other results are available from the design tool procedures. These are summarized here:

\begin{tabular}{|c|c|}
\hline $\mathrm{N}_{\mathrm{s}}$ & Specific speed \\
\hline$\Delta \mathrm{P}_{0}$ & Stator pressure loss \\
\hline$\Delta \mathrm{C}_{\theta}$ & Change in circumferential velocity required for work extraction \\
\hline $\mathrm{U}$ & Blade speed (hub, meanline, and tip) \\
\hline
\end{tabular}

Table B.5: General design outputs

\begin{tabular}{|c|c|}
\hline$T$ & Static temperature \\
\hline$M$ & Mach number (hub and tip) \\
\hline$M_{\text {rel }}$ & Relative Mach number (hub and tip) \\
\hline A & Flow area \\
\hline HTR & Hub-to-tip ratio \\
\hline
\end{tabular}

Table B.6: Additional station outputs

\begin{tabular}{|c|c|}
\hline$\sigma$ & Solidity \\
\hline $\mathrm{s}$ & Blade spacing \\
\hline $\mathrm{o}$ & Throat opening \\
\hline $\mathrm{h}$ & Blade height \\
\hline $\mathrm{i}$ & Incidence \\
\hline$\delta$ & Deviation \\
\hline $\mathrm{Re}$ & Reynolds number based on chord \\
\hline $\mathrm{t}_{\max }$ & Maximum blade thickness \\
\hline
\end{tabular}

Table B.7: Stator and rotor outputs 


\begin{tabular}{|c|c|}
\hline$Y^{\prime}{ }_{P}$ & Profile loss coefficient of AMDC \\
\hline$Y_{\text {shock }}$ & Shock loss coefficient \\
\hline$C F M$ & Drag rise factor \\
\hline$Y_{p}$ & Profile loss coefficient \\
\hline$f_{R e}$ & Reynolds number correction factor \\
\hline$Y_{T E T}$ & Trailing edge loss coefficient \\
\hline$Y^{\prime}$ & Secondary loss coefficient of AMDC \\
\hline$Y_{S}$ & Secondary loss coefficient \\
\hline$Y_{T o}$ & Total loss coefficient for zero tip clearance loss \\
\hline$Y_{T}$ & Total loss coefficient \\
\hline$\Delta \eta$ & Change in efficiency due to tip clearance losses \\
\hline
\end{tabular}

Table B.8: Performance estimation outputs

\begin{tabular}{|c|c|}
\hline$R$ & Average radius (hub and tip) \\
\hline$A_{\mathrm{ratio}}$ & Area ratio (defined in text) \\
\hline $\mathbf{t}$ & Time to creep rupture \\
\hline$\sigma_{\mathrm{gb}}$ & Gas bending stress \\
\hline$\sigma_{\mathrm{r}}$ & Radial stress distribution \\
\hline$\sigma_{\mathrm{t}}$ & Tangential stress distribution \\
\hline$\sigma_{\mathrm{vM}}$ & von Mises stress distribution \\
\hline $\mathrm{x}, \mathrm{y}, \mathrm{x}_{\mathrm{p} \ldots}$ & Blade attachment design results (see Figure 3.5) \\
\hline $\mathrm{N}_{\text {burst }}$ & Rotor burst speed \\
\hline $\mathrm{t}_{\text {casing }}$ & Minimum casing thickness \\
\hline
\end{tabular}

Table B.9: Structural evaluation outputs 


\section{Appendix C1 - Curve Fits for Axial Turbine Design and Analysis}

Figure 3.3(a) illustrates the stagger angle as a function of blade inlet and outlet flow angles. According to Sjolander (2002), the graphical data may be fitted to a surface, using a polynomial of the form:

$$
\Phi=\mathrm{a}_{0,0}+\sum \mathrm{a}_{\mathrm{i}, \mathrm{j}} \beta_{2}{ }^{\mathrm{i}} \beta_{1}{ }^{\mathrm{j}}
$$

with coefficients,

$$
\begin{array}{lll}
\mathrm{a}_{0,0}=-2.90463 & \mathrm{a}_{0,1}=0.307036 & \mathrm{a}_{0,2}=0.370176 \mathrm{E}-2 \\
\mathrm{a}_{1,0}=0.412797 & \mathrm{a}_{1,1}=-0.355369 \mathrm{E}-1 & \mathrm{a}_{1,2}=-0.194938 \mathrm{E}-3 \\
\mathrm{a}_{2,0}=0.593956 \mathrm{E}-2 & \mathrm{a}_{2,1}=0.389157 \mathrm{E}-3 & \mathrm{a}_{2,2}=1.74147 \mathrm{E}-6
\end{array}
$$

Figures 3.5(a) and 3.5(b) illustrate the profile loss coefficient used in the proposed loss correlation system. The data of both figures have been fitted to polynomials by Sjolander (2002) of the form:

$$
Y_{P}=a_{0,0}+\sum a_{i, j} \alpha_{2}{ }^{i}\left(\frac{s}{c}\right)^{j}
$$

For the nozzle blades $\left(\alpha_{1}=0\right)$, the values of the coefficients are as follows:

$$
\begin{aligned}
& \mathrm{a}_{0,0}=0.358716 \\
& \mathrm{a}_{0,1}=-1.43508 \\
& \mathrm{a}_{0,2}=1.57161 \\
& \mathrm{a}_{0,3}=-0.496917 \\
& \mathrm{a}_{1,1}=-0.0112815 \\
& \mathrm{a}_{1,2}=0.0548594 \\
& \mathrm{a}_{1,3}=0.014165 \\
& \mathrm{a}_{2,0}=0.000175083 \\
& \mathrm{a}_{2,1}=-0.000824937 \\
& \mathrm{a}_{2,2}=0.000652287 \\
& \mathrm{a}_{2,3}=-7.30141 \mathrm{E}-05 \\
& \mathrm{a}_{3,0}=-8.61323 \mathrm{E}-07 \\
& \mathrm{a}_{3,1}=3.95998 \mathrm{E}-06 \\
& \mathrm{a}_{3,2}=-1.89698 \mathrm{E}-06 \\
& \mathrm{a}_{3,3}=-4.9954 \mathrm{E}-07
\end{aligned}
$$

For the impulse blade rows $\left(\alpha_{1}=\alpha_{2}\right)$, the values of the coefficients are as follows:

$$
\begin{aligned}
& \mathrm{a}_{0,0}=0.0995503 \\
& \mathrm{a}_{0,1}=0.182837 \\
& \mathrm{a}_{0,2}=0.01603 \\
& \mathrm{a}_{1,0}=0.00621508 \\
& \mathrm{a}_{1,1}=-0.0283658 \\
& \mathrm{a}_{1,2}=0.011249 \\
& \mathrm{a}_{2,0}=-7.10628 \mathrm{E}-05 \\
& \mathrm{a}_{2,1}=0.000327648 \\
& \mathrm{a}_{2,2}=-0.000122645
\end{aligned}
$$




\section{Appendix D1 - Blade Geometry with Tip Clearance in ANSYS Workbench 9.0}

\section{Start ANSYS Workbench 9.0}

Select new Geometry

\section{Specify desired units}

Select Millimeters and Okay

This will open the DesignModeler workspace

\section{Import geometry}

Select File > Import External Geometry File > Select the appropriate *.bgd file ( $g g$ rotor_combo.bgd) and click Open

Click the Generate button in the DesignModeler workspace

This will complete the import process. A solid fluid domain should be viewable with a void where the blade would be.

\section{Fill blade void}

\section{Select Tools $>$ Fill}

Select the 4 faces which define the blade void

Use the Ctrl key to select multiple faces and use the middle mouse button to rotate In the "Details of Fill1" entry box click Apply

Click the Generate button again

This will create a "frozen" solid in place of the blade void.

\section{Create thin surface}

Select the Thin/Surface button located just above the viewing window Select the top surface of the "frozen" solid blade In the "Details of Fill1" entry box click Apply Under "Details of Thin1" change Selection Type to "Faces to keep" and Thickness to $0.6 \mathrm{~mm}$

Click Generate

\section{Unfreeze tip clearance surface}

Select Tools $>$ Unfreeze

Select the "frozen" blade tip and click Apply

Once again click Generate 


\section{Save DesignModeler file}

File $>$ Save As...

Select a name for the file ( $g g$ rotor_combo.agdb) and click Okay

\section{Summary:}

This will generate a solid model of the fluid domain to be analyzed using CFX-5. The next step is to generate the mesh for this blade passage. By rotating the solid model in the ANSYS Workbench workspace, one can see the effect of filling in the top $0.6 \mathrm{~mm}$ of the blade void. To create the stator geometry, or a rotor blade without tip clearances, repeat the above process omitting steps 4,5 , and 6 . 


\section{Appendix D2 - Mesh generation in ANSYS Workbench 9.0}

\section{Open CFX-Mesh}

CFX-Mesh may be opened from the ANSYS Workbench start screen; however, it is more convenient to open it from the DesignModeler Tasks tray. Continuing from Appendix D1...

Close the DesignModeler workspace and select "Generate CFX Mesh" from the Tasks tray located on the left side of the screen

\section{Create 2D Regions}

Right click on Regions in the model tree

Select Insert $>$ Composite 2D Region

Rename the Region "Inlet" and select the inlet face defining the flow domain inlet Click Apply

Repeat this process 5 additional times for the Outlet, Hub, Shroud, Periodic Master, and Periodic Slave. Leave the 5 faces defining the blade walls as the

"Default 2D Region"

\section{Set global mesh parameters}

Under Default Body Spacing, change Maximum Spacing to $2 \mathrm{~mm}$

Under Default Face Spacing, change Maximum Edge Length to $2 \mathrm{~mm}$

\section{Create local mesh controls}

Right click on Spacing

Select Insert $>$ Face Spacing

Rotate the solid so that the blade void is visible

Select the top face and click Apply

Change the drop down menu next to Face Spacing Type to Angular Resolution

Set Maximum Edge Length to $0.2 \mathrm{~mm}$

Add an additional Face Spacing control to the other 4 blade walls

Select Insert $>$ Face Spacing

Select the 4 blade walls and click Apply

Change the drop down menu next to Face Spacing Type to Angular Resolution

Set Maximum Edge Length to $0.75 \mathrm{~mm}$

Set Expansion Factor to 1.4

5. Create a periodic boundary

Right click on Periodicity 
Select Insert $>$ Periodic Pairs

Select Rotational from the drop down menu next to Periodic Type

Click the highlighted region next to Location 1 and select one of the two faces which define the periodic pair (this should be same face used to define the

Periodic Master 2D Region of Step 2)

Click Apply

Select the other periodic face

Click Apply

\section{Insert inflation layers}

Right click on Inflation

Select Insert $>$ Inflated Boundary

Select the 4 faces defining the blade profile, excluding the blade tip

Set the Maximum Thickness to $0.6 \mathrm{~mm}$

Again select Insert > Inflated Boundary

Select the face defining the blade tip

Set the Maximum Thickness to $0.2 \mathrm{~mm}$

\section{Save}

Before generating the mesh, be sure to save what has been done to this point. Sometimes the meshing program can crash; causing all of the settings that have been modified will be lost.

File $>$ Save As...

If all the steps are followed exactly, the name should be the same as that entered for the model geometry of Appendix D1. In this case, the name should read gg_rotor_combo.cmdb

\section{Mesh}

From the drop down menu

Select Go > Generate Volume Mesh

Rename the file gg_rotor_combo_meshl.gtm

This file is now ready for use in CFX-5

\section{Summary:}

Using the steps outlined above, a variety of meshes can be generated with varying densities and inflation layers. Simply change the values of the various parameters. The quality of the mesh can not be viewed directly in ANSYS Workbench. To view the mesh, open the *.gtm file in CFX-Post. 
Open CFX-Post

File $>$ Load Results

Select the appropriate *.gtm file

From the top menu select:

Create $>$ Object $>$ Plane

Beside Method select $Y Z$ Plane and set the $X$ distance to approximately $0.13 \mathrm{~m}$

Select the Colour tab

Set the Colour to Black

Select the Render tab

Check the Draw Lines option

Repeat for an XY Plane if desired

To determine the number of nodes and elements

Select Tools $>$ Mesh Calculator

Select Mesh Statistics from the Function option and click Calculate 


\section{Appendix D3 - Stator-Rotor Simulation Setup in CFX-Pre}

The methods presented here will continue on the Development of a complete stage analysis using CFX-5. The setup will include the use of the geometry and mesh files created in Appendix D1 and C2.

Start the CFX 5.7.1 Launcher and select CFX-Pre 5.7.1

\section{Create a new simulation}

Specify a File name gg_combo.cfx

Select Turbo and Save

This will open the Turbo wizard

\section{Component definition}

Leave the Axes and Simulation Type as default

Click Next

\section{Stator stage definition}

Select New

Change the domain name to Stator 1

Leave Component Type as Stationary, and select the stator mesh by clicking the icon next to Select Mesh

Specify CFX-5 GTM file for Mesh Format and click the icon next to File

Select gg_stator_combo.gtm, or the equivalent. (Note, the mesh generation for this file has not been described. It is assumed the reader will generate their own by following the methods of Appendices D1 and D2)

Click Open and Okay

This will import the specified mesh file

Beside Select Mesh, select Assembly from the drop down menu

\section{Rotor stage definition}

Select New

Change the domain name to Rotor 1

Change Component Type to Rotating

Set the Speed to $-35000 \mathrm{rev} \mathrm{\textrm {min } ^ { - 1 }}$

Select the stator mesh by clicking the icon next to Select Mesh

Specify CFX-5 GTM file for Mesh Format and click the icon next to File 
Click the icon next to Select Mesh, change the Mesh Format to CFX-5 GTM file Open the appropriate *gtm file. This time use the gg_rotor_combo_meshl.gtm file generated in Appendix D2.

Beside Select Mesh, select Assembly 2 from the drop down menu

(Note that the mesh assemblies will typically lie on top of each other in this case. This is a result of the coordinate system used to generate the blade profiles in CFX-BladeGen.)

\section{Translate assemblies}

Expand the Mesh Tools dialogue box and select Assembly 2 from the Transform drop down menu

Click the icon next to Transform

Select Translation from the Transformation drop down menu

Enter $0.0326 m$ for $D z$

Click Okay

Click Next

\section{Define interfaces}

Delete the default Interface connections

To create the stator-rotor domain interface

Select New

Rename to Stator-Rotor Interface

Set Side 1 to Inlet 2 and Side 2 to Outlet

Set Type to Stage

To create the periodic boundary conditions

Select New

Rename to Stator Periodic

Set Side 1 to Periodic Master and Side 2 to Periodic Slave

Set Type to Periodic

Select New

Rename to Rotor Periodic

Set Side 1 to Periodic Master 2 and Side 2 to Periodic Slave 2

Set Type to Periodic

Click Next

\section{General physics}

Set Reference Pressure to $0 \mathrm{~atm}$ 


\section{Click Next}

\section{Boundary definition}

Under Existing Boundaries select Inlet

Next to Option under Flow Specification select Stationary Frame Total Pressure Enter $763000 \mathrm{~Pa}$ for the Relative Pressure

Next to Total Temperature enter $1200 \mathrm{~K}$

Under Existing Boundaries select Outlet

Ensure Average Static Pressure is specified next to Option under Flow

Specification

Enter $220000 \mathrm{~Pa}$ for the Relative Pressure

Click Next

Click Finish

The General Mode of CFX-Pre will now be activated. Occasionally, some of the settings specified in the Turbo wizard are not carried over to the General Mode. Typically, a warning is displayed; however, it is important to double check these settings before running a solution.

\section{Relative wall motion}

Open the Shroud boundary condition under Rotor 1 in the model tree Select the Boundary Details tab

Check the box next to Wall Velocity and select Rotating Wall from the drop down menu next to Option

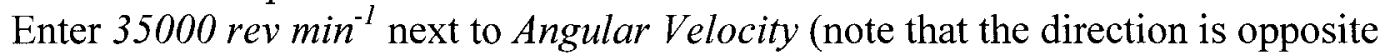
the rotor rotational rate entered in Step 4)

Click Okay

\section{Turbulence and heat transfer models}

Double click Domain Models in the model tree

Click the Fluid Models tab

Under Heat Transfer Model, check the box next to Include Viscous Work Term

Under Turbulence Model, select Shear Stress Transport from the drop down

menu next to Option

Click Okay

\section{Solver control}

Double click Solver Control in the model tree

Change Max. No. Iterations to $10^{6}$

Change Residual Type to MAX 


\section{Output control}

The user may use the controls under this heading for backing up results or outputting additional variables to the results files. See the help manual for additional information.

\section{Fluid properties}

Double click Air Ideal Gas located at the bottom of the model tree

Select the Material Properties tab and scroll down to Specific Heat Capacity Enter $1154\left[\mathrm{~J} \mathrm{~kg}^{-1} \mathrm{~K}^{-1}\right]$

\section{Save and Solve}

Save the simulation and write the definition file

File $>$ Save As...

File $>$ Write Solver (.def) File...

A new dialogue box will appear, unselect Quit CFX-Pre

Click Okay

The Solver Manager will be started

\section{Summary:}

This will provide the CFX-5 solver a complete simulation file which may be used for validating the output of the design tool described earlier. Modifications of the various settings were also conducted. The results are described in Chapter 6. 


\section{Appendix D4 - Creation of Rotor Solid Model in Pro/ENGINEER}

This appendix will describe the creation of a rotor solid model in Pro/ENGINEER Wildfire.

1. Create a New part using the Pro/ENGINEER defaults

2. Change the units

From the drop down menu select

Edit $>$ Setup $>$ Units $>m m N s>$ Set $>$ Same Dims

\section{Create an axis of revolution}

Select planes TOP and RIGHT

From the drop down menu select

Insert $>$ Model Datum $>$ Axis

4. Create a datum plane

From the drop down menu select

Insert $>$ Model Datum > Plane

Select the $A_{-} 1$ and TOP

Set Rotation $=5^{\circ}$

Click Okay

5. Create a new coordinate system

From the drop down menu select

Insert $>$ Model Datum $>$ Coordinate System

Select DTMI, RIGHT, and FRONT as references

Click the Orientation tab

Align the $Z$ axis along $A_{-} 1$

6. Import the blade curves

From the drop down menu select

Insert $>$ Model Datum $>$ Curve $>$ From File $>$ Done

Select $C S O$ as the coordinate system

Open the *.ibl BladeGen output file

7. Create additional curves for the blade tips

From the drop down menu select

Insert $>$ Model Datum $>$ Curve $>$ Thru Points $>$ Done 
Select the two points that connect the blade tip leading edge to the remaining profile

Select $O k a y>$ Done $>$ Okay

Repeat for the blade trailing edge

\section{Create blade surfaces}

Insert $>$ Boundary Blend...

Select a minimum of two curves defining the surface chains in the first direction Click the Second Direction Chain Selector

Select a minimum of two curves defining the surface chains in the second direction

Repeat this process for each of the 4 blade surfaces, omitting the hub and tip

\section{Create tip surface}

For the blade leading and trailing edge surfaces only one direction needs to be specified

Insert $>$ Boundary Blend...

Select the curve created in Step 7 and the blade leading edge

Click Okay

Repeat for the trailing edge tip

Create an additional surface for large section of the blade tip as per Step 8

\section{Merge Surfaces}

Select two adjacent Boundary Blends from the model tree window

From the drop down menu select

Edit $>$ Merge $>$ Okay

Select Mergel and the next adjacent Boundary Blend

Again from the drop down menu select

Edit $>$ Merge $>$ Okay

Repeat until all of the Boundary Blends have been merged

\section{Create a datum plane}

From the drop down menu select

Insert $>$ Model Datum > Plane

Select the $A_{-} 1$ and $R I G H T$

Set Rotation $=5^{\circ}$

Click Okay

\section{Create the rotor disk}

From the drop down menu select 
Insert $>$ Revolve...

From the bottom tool bar select

Placement $>$ Define...

Next to Plane select DTM2 (created in Step 11)

Next to Reference select DTM1 (created in Step 4)

Click Sketch

Select any coordinate frame

Click Okay and Close

Within the sketcher tool select

Sketch $>$ Edge $>$ Use...

Select the hub datum curve

Close the section with a $2 \mathrm{D}$ profile of the rotor disk

Exit sketcher

Select axis $A \quad l$ and revolve $360^{\circ}$

\section{Pattern blades}

In the model tree window select all features from $D T M 1$ to $M E R G E 6$

Right click on the selected features

Select Group

Right click on Group LOCAL GROUP

Select Pattern...

Select the angular offset of DTMI (currently $5^{\circ}$ ) and enter $360 / n$

(where $\mathrm{n}$ is the number of rotor blades to pattern)

Specify the number of copies, $n$

\section{Solidify blades and disk}

Merge each of the copied blades to the revolved section created in Step 12

Select the final Merge from the model tree

From the drop down menu select

Edit $>$ Solidify

\section{Create fractional model}

A fractional model may be created to save computational time for finite element analysis

From the drop down menu select

Insert $>$ Extrude...

Create a pie shaped extrusion perpendicular to axis A_ 1

Specify extrusion direction

Select Remove Material and Okay 


\section{Appendix D5 - Model setup in ANSYS 9.0}

This appendix will describe the procedure for setting up a model for analysis in ANSYS 9.0. The procedure will guide the user from start through mesh generation as these requirements are shared by all of the analyses conducted. The specific methods required for inertial, thermal, or vibration analyses are presented in Appendices D6, D7, and D8.

\section{Import the geometry}

Open the solid model in Pro/ENGINEER Wildfire

From the drop down menu select

ANSYS $9.0>$ ANSYSGeom $>O K$

This will open the ANSYS Multiphysics Utility window

From the drop down menu select

Plot $>$ Volumes

\section{Specify a new jobname and working directory}

From the drop down menu select

File $>$ Change Jobname...

Enter a new jobname and select $O K$

File $>$ Change Directory

Select a working directory and $O K$

Save database

File $>$ Save as Jobname.db

\section{Specify element type}

From the ANSYS Main Menu (located at the left side of the screen) select

Preprocessor $>$ Element Type $>$ Add/Edit/Delete...

Select Add...

Select Structural Mass $>$ Solid $>$ Brick 20 node 95

$O K$ and Close

\section{Specify material properties}

From the ANSYS Main Menu select

Preprocessor $>$ Material Props $>$ Material Models...

Select Structural $>$ Linear $>$ Elastic $>$ Isotropic

For $E X$ enter 173530

For PRXY enter 0.317

Click $O K$

Select Structural $>$ Density

For DENS enter 8.19e-9

Click $O K$ 
Select Structural $>$ Thermal Expansion $>$ Secant Coefficient $>$ Isotropic

For Reference temperature enter 298

For $A L P X$ enter $14.4 e-6$

Click $O K$

Select Thermal $>$ Conductivity $>$ Isotropic

For $K X X$ enter 20

Click $O K$ and close window

\section{Add cyclic constraint}

This step is only required for a fractional model analysis

From the ANSYS Main Menu select

Preprocessor $>$ Modeling $>$ Cyclic Sector $>$ Cyclic Model $>$ Auto Defined $>O K$

\section{Mesh model}

From the ANSYS Main Menu select

Preprocessor > Meshing > Mesh Tool...

Check the box next to Smart Size

Select desired grid density $1-10$

Click Mesh

Select the volume to be meshed

Click $O K$

The meshing process within ANSYS can be very frustrating, especially with complex shapes. It will sometimes be necessary to divide up the volume and mesh the sub volumes independently. It is also possible to set local size controls on various areas such as fillets or highly curved surfaces. Practice generating various mesh densities on various shapes using the options available through the Mesh Tool. Further information is available in the help manual. 


\section{Appendix D6 - Inertial analysis in ANSYS 9.0}

This appendix will describe the procedure for setting an inertial analysis in ANSYS 9.0. The appendix serves as a continuation to the model setup presented in Appendix D5.

\section{Change active coordinate system}

From the drop down menu select

Workplane $>$ Change Active CS to $>$ Global Cylindrical

2. Specify analysis type

From the ANSYS Main Menu select

Preprocessor $>$ Loads $>$ Analysis Type $>$ New Analysis $>$ Static $>O K$

\section{Apply loads}

From the ANSYS Main Menu select

Preprocessor $>$ Loads $>$ Define Loads $>$ Apply $>$ Structural $>$ Displacement $>$

On Areas...

Select the end of the rotor shaft

Click $O K$

Select All DOF and enter 0 next to VALUE

Click $O K$

From the ANSYS Main Menu select

Preprocessor $>$ Loads $>$ Define Loads $>$ Apply $>$ Structural $>$ Inertia $>$ Angular Veloc $>$ Global...

Enter 3665 (rotational rate for gas generator in $\mathrm{rad} / \mathrm{s}$ ) next to $O M E G Z$

Click $O K$

\section{Solve}

From the ANSYS Main Menu select

Solution $>$ Solve $>$ Current $L S>O K$

\section{Post process the results}

From the ANSYS Main Menu select

General Postproc $>$ Read Results $>$ First Set

General Postproc > Plot Results... 


\section{Appendix D7 - Thermal analysis in ANSYS 9.0}

This appendix will describe the procedure for setting up a thermal analysis in ANSYS 9.0. The appendix serves as a continuation to the model setup presented in Appendix D5. This analysis is done in two parts. First, an analysis is conducted for the temperature distribution within the component. Second, the temperature results are applied as a load to solve for the stress distribution.

\section{Change element type}

From the ANSYS Main Menu select

Preprocessor > Element Type > Switch Elem Type...

From the drop down menu select

Struc to Thermal

\section{Change active coordinate system}

From the drop down menu select

Workplane $>$ Change Active CS to $>$ Global Cylindrical

\section{Specify analysis type}

From the ANSYS Main Menu select

Preprocessor $>$ Loads $>$ Analysis Type $>$ New Analysis $>$ Static $>O K$

\section{Apply temperature constraints}

From the ANSYS Main Menu select

Preprocessor $>$ Loads $>$ Define Loads $>$ Apply $>$ Thermal $>$ Temperature $>$ On Areas...

Select the blade leading edge

Click $O K$

Select Temp and enter 1055 next to VALUE

Click $O K$

Preprocessor $>$ Loads $>$ Define Loads $>$ Apply $>$ Thermal $>$ Temperature $>$

On Areas...

Select the blade trailing edge

Click $O K$

Select Temp and enter 945 next to VALUE

Click $O K$

\section{Solve and save database}

From the ANSYS Main Menu select

Solution $>$ Solve $>$ Current $L S>O K$

Once the solution is complete 
File $>$ Save as Jobname. $d b$

5. Change jobname

File > Change Jobname

6. Change element type

From the ANSYS Main Menu select

Preprocessor $>$ Element Type $>$ Switch Elem Type...

From the drop down menu select

Thermal to Struc

\section{Apply temperature load}

From the ANSYS Main Menu select

Preprocessor $>$ Loads $>$ Define Loads $>$ Apply $>$ Temperature $>$ From Therm Analy...

Click Browse and select the appropriate *.rth (thermal results) file generated in Step 4

Click $O K$

\section{Solve}

From the ANSYS Main Menu select

Solution $>$ Solve $>$ Current $L S>O K$

\section{Post process the results}

From the ANSYS Main Menu select

General Postproc $>$ Read Results $>$ First Set

General Postproc $>$ Plot Results... 


\section{Appendix D8 - Vibration analysis in ANSYS 9.0}

This appendix will describe the procedure for setting up a vibration analysis for a single blade in ANSYS 9.0. The appendix serves as a continuation to the model setup presented in Appendix D5.

\section{Specify analysis type}

From the ANSYS Main Menu select

Preprocessor $>$ Loads $>$ Analysis Type $>$ New Analysis $>$ Modal $>O K$

Preprocessor $>$ Loads $>$ Analysis Type $>$ Analysis Options...

In the Modal Analysis window

Next to No. of modes to extract enter 10

Check the box next to Calculate elem results

Click $O K$

\section{Constrain the blade root}

From the ANSYS Main Menu select

Preprocessor $>$ Loads $>$ Define Loads $>$ Apply $>$ Structural $>$ Displacement $>$

On Areas...

Select the blade root

Click $O K$

Select $A l l D O F$ and enter 0 next to $V A L U E$

Click $O K$

4. Solve

From the ANSYS Main Menu select

Solution $>$ Solve $>$ Current $L S>O K$

\section{Post process the results}

From the ANSYS Main Menu select

General Postproc $>$ Results Summary

General Postproc $>$ Read Results $>$ First Set 